Florida International University FIU Digital Commons

$7-27-2012$

\title{
Ethnic Identification: Its Impact on HIV/AIDS Risk among Migrant and Seasonal Workers in South Florida
}

Nancy Shehadeh

Florida International University, nancyshehadeh@gmail.com

DOI: $10.25148 /$ etd.FI12110708

Follow this and additional works at: https://digitalcommons.fiu.edu/etd

\section{Recommended Citation}

Shehadeh, Nancy, "Ethnic Identification: Its Impact on HIV/AIDS Risk among Migrant and Seasonal Workers in South Florida" (2012). FIU Electronic Theses and Dissertations. 722.

https://digitalcommons.fiu.edu/etd/722 


\section{FLORIDA INTERNATIONAL UNIVERSITY}

Miami, Florida

ETHNIC IDENTIFICATION: IMPACT ON HIV/AIDS RISK AMONG

MIGRANT AND SEASONAL WORKERS IN SOUTH FLORIDA

A dissertation submitted in partial fulfillment of the

Requirements for the degree of

DOCTOR OF PHILOSOPHY

in

PUBLIC HEALTH

by

Nancy Shehadeh

2012 
To: Interim Dean Michele Ciccazzo

Robert Stempel College of Public Health and Social Work

This dissertation, written by Nancy Shehadeh, and entitled Ethnic Identification: Its Impact on HIV/AIDS Risk among Migrant and Seasonal Workers in South Florida, having been approved in respect to style and intellectual content, is referred to you for judgment.

We have read this dissertation and recommend that it be approved.

$\begin{array}{r}\hline \text { Jesus Sanchez } \\ \hline \text { Mark Williams } \\ \hline \text { Mary Jo Trepka } \\ \hline \text { H. Virginia McCoy, Major Professor }\end{array}$

Date of Defense: July 27, 2012

The dissertation of Nancy Shehadeh is approved.

Interim Dean Michele Ciccazzo Robert Stempel College of Public Health and Social Work

Dean Lakshmi N. Reddi University Graduate School

Florida International University, 2012 


\section{DEDICATION}

I dedicate this degree to my dearest family. I would like to start with my extremely supportive and patient husband. He had to endure all the obstacles and breakdowns that I had on the way to completing this degree. Thank you, Marwan, for believing in me more than I believed in myself. Thank you, my beautiful children, Layaly, Ramsey, and Rami, who put up with my studying all these years and always wondered if mom would ever finish school. Last, but not least, a special dedication to my parents, Lutfi and Nadera Shehadeh, for believing in my potential in completing this degree. I want to thank my mother for instilling the importance of education in me at a very young age. And a very special thanks to my father who gave me a motivational speech every morning on the phone regarding the importance of completing my $\mathrm{PhD}$ and to never give up. I love you all so much!! 


\section{ACKNOWLEDGMENTS}

I gratefully acknowledge the support and encouragement of my mentor and major professor, Dr. H. Virginia McCoy, whose belief in my abilities helped get me through the requirements of this degree. I truly cherish her friendship and guidance through this long and arduous process. I would like to thank Dr. Mary Jo Trepka for her expertise and patience in supervising the statistical and methodological portion of this research. I would like to thank Dr. Jesus Sanchez for all his guidance and availability for questions throughout the dissertation process. Dr. Williams, thank you for all your time and guidance through the dissertation. I will not forget all the friendly support and advice I received from my dear friend, Dr. Terri Jennings. 


\begin{abstract}
OF THE DISSERTATION
ETHNIC IDENTIFICATION: ITS IMPACT ON HIV/AIDS RISK AMONG

MIGRANT AND SEASONAL WORKERS IN SOUTH FLORIDA
\end{abstract}

by

Nancy Shehadeh

Florida International University, 2012

Miami, Florida

Professor H. Virginia McCoy, Major Professor

Migrant workers are one of the most rapidly growing populations in the United States (U.S.) and have been significantly affected by HIV/AIDS. More than 9 million people in the U.S., primarily concentrated in Texas, Florida, Washington, California, Oregon, and North Carolina, are migrant farm workers. High prevalence rates are also suspected among migrant worker communities where risky health behaviors appear to be common. Constant mobility, isolation, limited education, substandard housing, and poverty are some of the factors that migrant workers experience and in many cases increases their HIV risk. Recent studies have suggested that ethnic identity or the level of attachment with one's ethnic group may influence engagement in HIV risk behaviors, a fact that may be important in the development of interventions among ethnic minorities. This study assesses the relationship between ethnic identity and HIV risk behaviors in two different samples; one assesses this relationship at baseline with a total of 431 African American migrant and seasonal workers in Immokalee, Florida. The second analyzes changes in ethnic identity and HIV behaviors in a sample of 270 Hispanic and African American migrant and seasonal workers in Immokalee, Florida. Data from 
baseline and 6-month follow-up were used in the analyses presented. The results suggest that individuals with higher levels of ethnic identity report lower levels of engagement in some, but not all, of the risky behaviors examined. These findings point to a potentially protective role for ethnic identity among this sample. 
INTRODUCTION

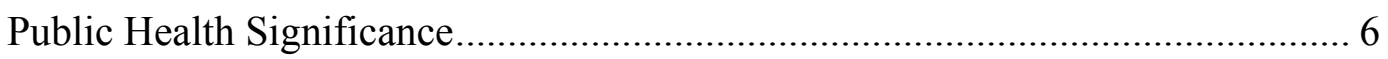

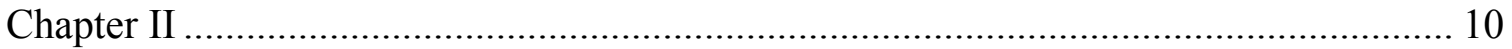

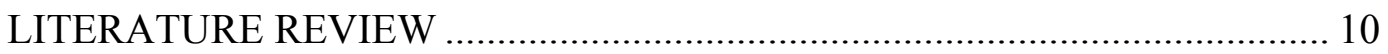

HIV/AIDS Background and Public Health Impact........................................... 10

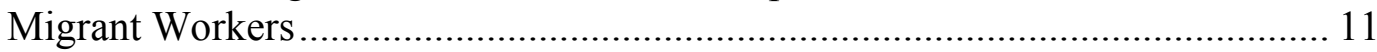

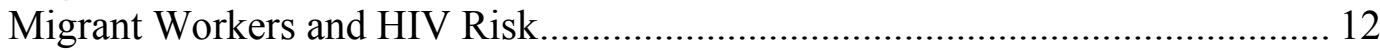

Ethnic Identity and Acculturation.................................................................... 15

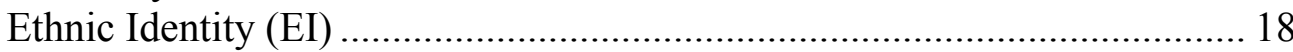

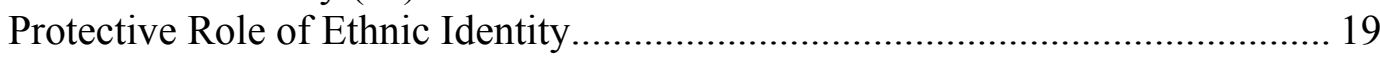

Ethnic Identity and Changes Over Time........................................................... 22

Ethnic Identity and HIV Risk Behaviors ............................................................ 23

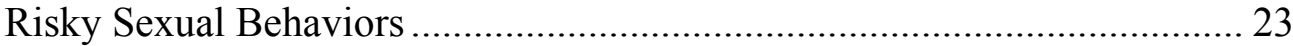

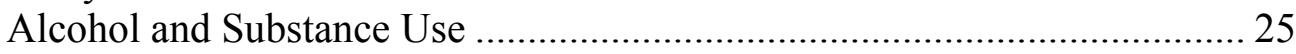

Moderating Variables on Ethnic Identity and HIV Risk ..................................... 27

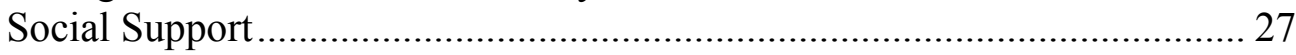

Social Support and Ethnic Identity …………........................................ 28

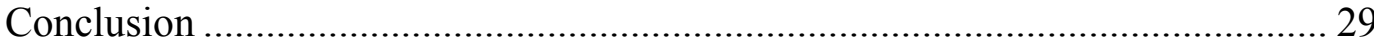

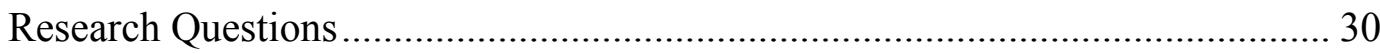

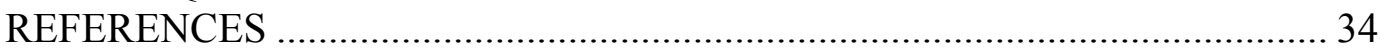

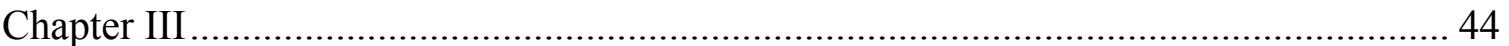

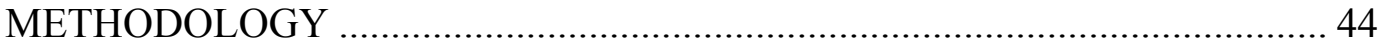

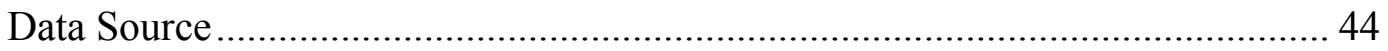

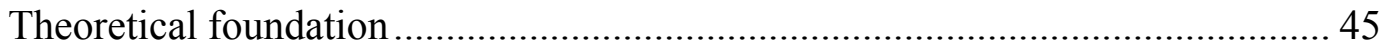

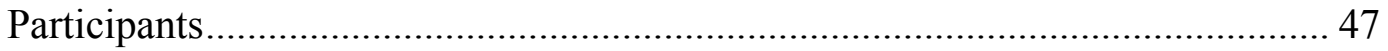

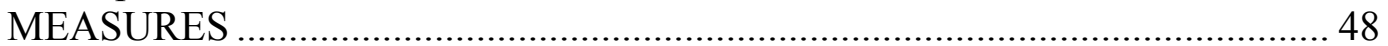

HIV/AIDS Risk Reduction Questionnaire (HRRQ) ………................................. 48

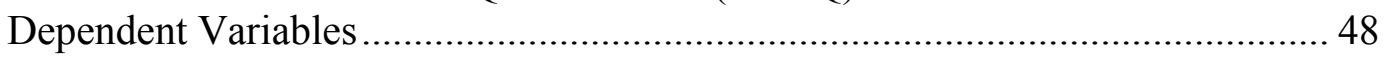

Sexual Risk: Vaginal Episode Equivalent (VEE) ......................................... 49

Sex-Related Alcohol Expectancies (SRAE) …………………………........ 50

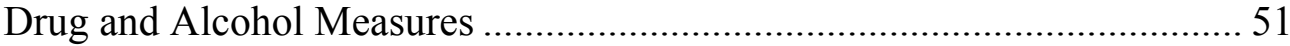

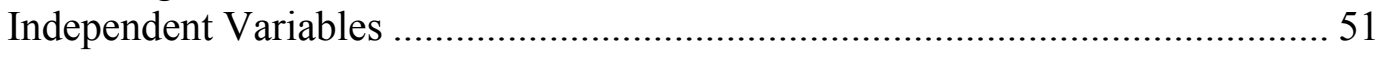

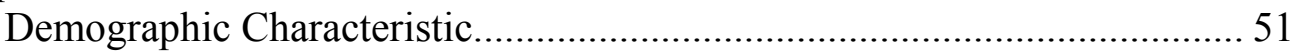

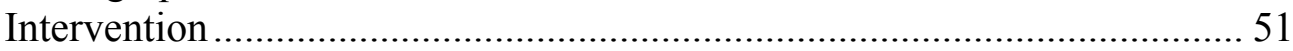

Ethnic Identity Measure: Multigroup Ethnic Identity Measure (MEIM) ..... 52

Social Support: Social Provisions Scale (SPS) ………………………........ 53

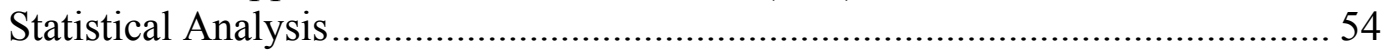

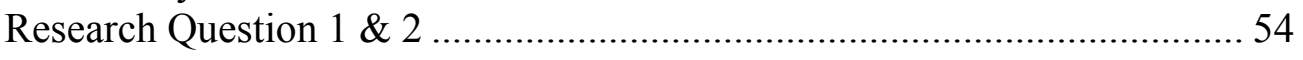

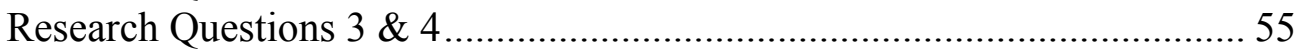

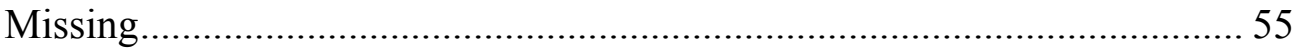




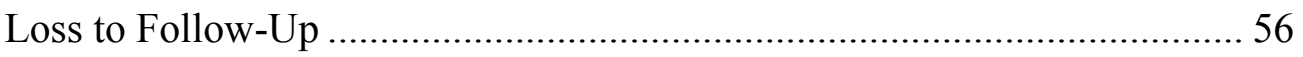

Sample Size and Power Analysis................................................................ 57

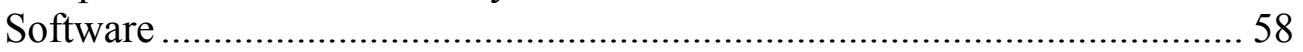

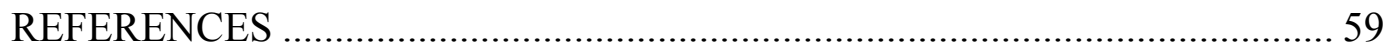

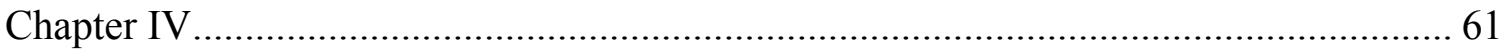

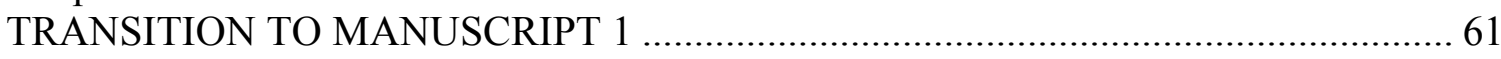

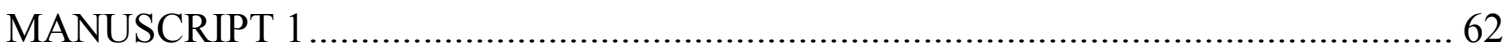

THE ROLE OF ETHNIC IDENTITY ON ALCOHOL AND DRUG USE AMONG

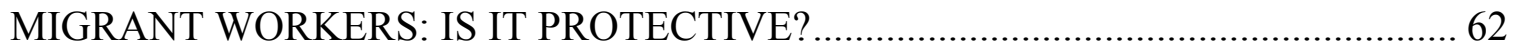

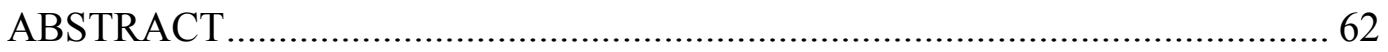

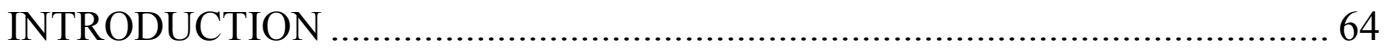

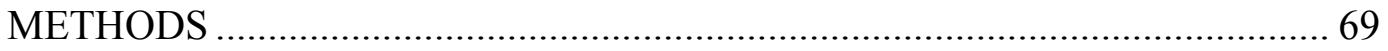

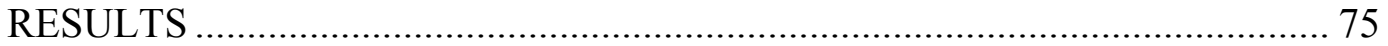

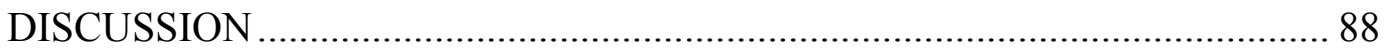

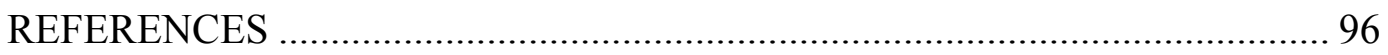

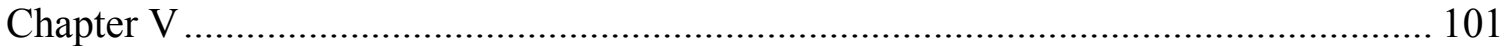

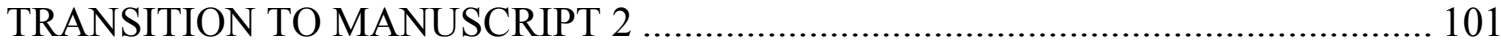

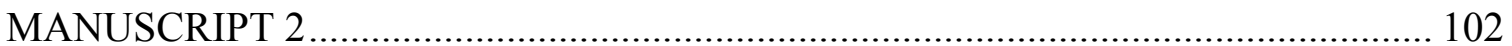

RISKY SEXUAL BEHAVIORS: THE IMPACT OF ETHNIC IDENTITY ON HIV

RISK BEHAVIORS AMONG MIGRANT AND SEASONAL WORKERS................. 102

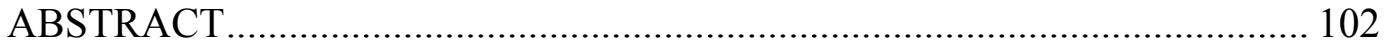

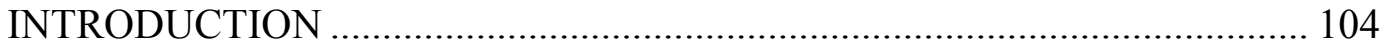

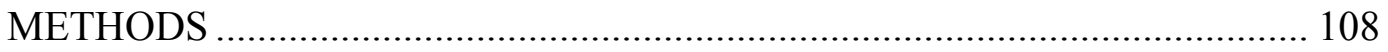

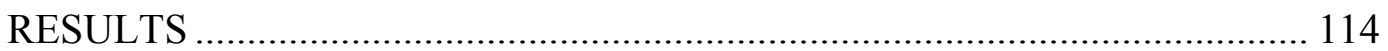

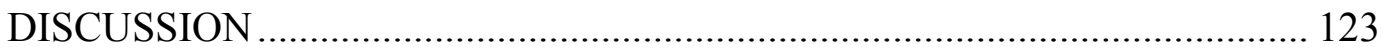

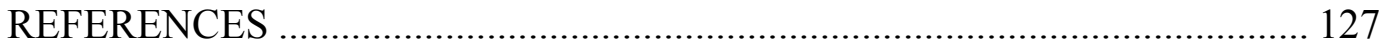

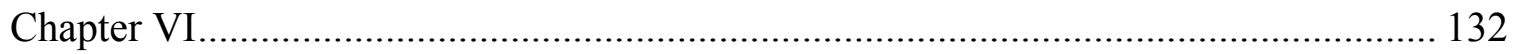

TRANSITION TO MANUSCRIPT 3: MODERATOR ANALYSIS ………………..... 132

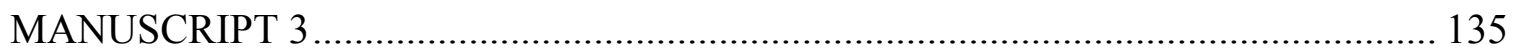

THE ROLE OF SOCIAL SUPPORT WITH ETHNIC IDENTITY AMONG

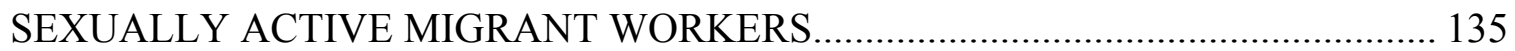

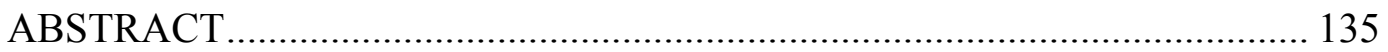

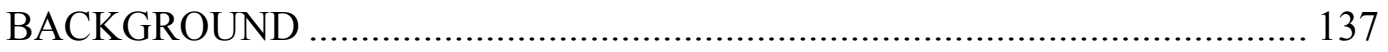

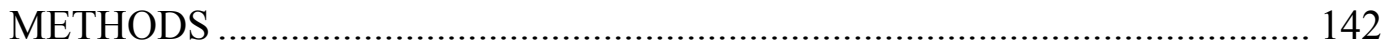

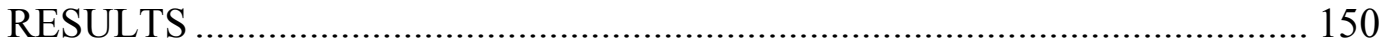

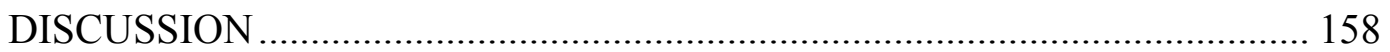

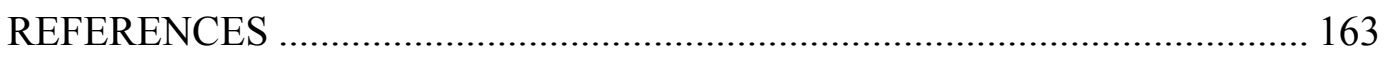

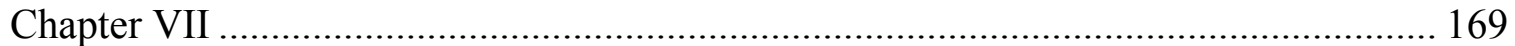

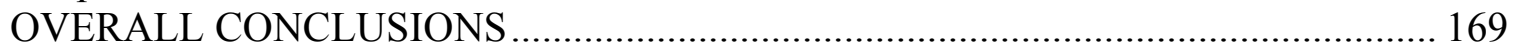

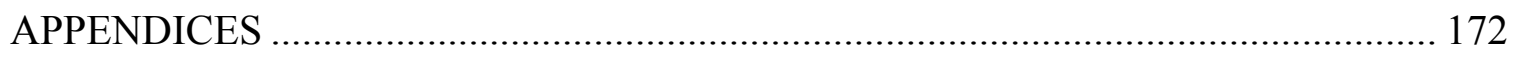

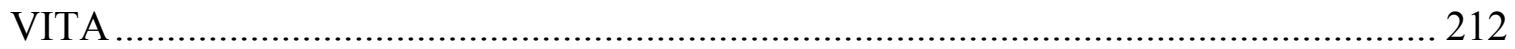




\section{LIST OF TABLES}

\section{MANUSCRIPT 1}

Table 1.1 (1) Baseline, 6 month follow-up, and Loss-to-Follow Up data sets by Demographics ........................................................................... 77

Table 1.2 (2) Differences in Ethnic Identity Explore and Ethnic Identity Belonging by demographics and Substance Use $(n=431 ; 6$ ths: $n=270) \ldots \ldots . .80$

Table 1.3 (3) Regression Analysis Summary for Ethnic Identity Predicting Alcohol and Drug use at Baseline $(n=431)$ and over 6 months $(n=270)$....... 84

Table 1.4 (4) Repeated Measures ANCOVA for Ethnic Identity ( $n=270)$ 87

\section{MANUSCRIPT 2}

Table 2.1 (5) Demographic Characteristics of Participants at Baseline ( $n=431), 6$ month follow-up $(n=270$, and Lost-to-Follow Up $(n=161) \ldots \ldots \ldots \ldots . . . .116$

Table 2.2 (6) Differences in Ethnic Identity Explore and Ethnic Identity Belonging by Demographics and Sexual Risk (n=431; 6 mths: $n=270))$......... 118

Table 2.3 (7) Regression Analysis Summary for Ethnic Identity Predicting Risky Sexual Behaviors at Baseline $(n=431)$

Table 2.4 (8) Repeated Measures Analysis of Covariance: Mean Scores and Standard Deviations for Ethnic Identity and Subscales at Baseline $(n=431)$ and 6 Months $(n=270)$

\section{MANUSCRIPT 3}

Table 3.1 (9) Baseline, 6 month follow-up, and Loss-to-Follow Up data sets by Demographic Characteristics.

Table 3.2 (10) Differences in Ethnic Identity Explore change and Ethnic Identity Belonging Change by Demographics and Social Support $(n=270) .153$ 
Table 3.3 (11) Regression analysis for Ethnic Identity and Social Support Predicting

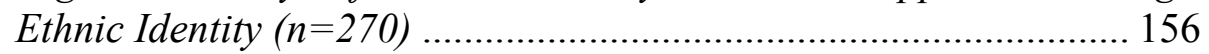

Table 3.4 (12) Repeated Measures Analysis of Covariance for Ethnic Identity Levels by Assignment of Intervention Group (PEER \& HEALTHY) $(n=270)$ 


\section{ACRONYMS AND ABBREVIATIONS}

$\begin{array}{ll}\text { HIV } & \text { Human Immunodeficiency Virus } \\ \text { AIDS } & \text { Acquired Immunodeficiency Syndrome } \\ \text { EI } & \text { Ethnic Identity } \\ \text { STI } & \text { Sexually Transmitted Infections } \\ \text { SPS } & \text { Social Provisions Scale } \\ \text { VEE } & \text { Vaginal Episode Equivalent } \\ \text { SRAE } & \text { Sex-Related Alcohol Expectancies } \\ \text { HRRQ } & \text { HIV Risk Reduction Questionnaire } \\ \text { RMANCOVA } & \text { Repeated Measures of Analysis of Covariance } \\ \text { MEIM } & \text { Multigroup Ethnic Identity Measure }\end{array}$




\section{Chapter I}

\section{INTRODUCTION}

Migrant workers were "the forgotten people," as Edward Murrow put it in the news documentary known as "Harvest of Shame," because they were overlooked by researchers for many years (Lowe, 1960). Migrant workers became a hot topic back in the 1960s when the U.S. population was made aware of the uncivilized conditions that migrant workers endured. The famous CBS journalist, Edward R. Murrow, created the documentary called "Harvest of Shame." This program was aired on Thanksgiving in 1960, and it depicted the agonizing conditions migrant workers experienced at work and in their homes. The actual farm work was labeled as "the sweat shops of the soil" (Lowe, 1960). Another documentary created by Byron Pitts, also a CBS journalist, updated the 1960 documentary in the year 2010. Unfortunately, not much improvement has been made in living or working conditions in this marginalized community (Pitts, 2010). Both documentaries were made in Immokalee, Florida, which is the same location in which the sample used in the studies lives.

Migrant workers are one of the most rapidly growing populations in the United States (U.S.) and have been significantly affected by HIV/AIDS (Coppel, Dumont, \& Visco, 2001; Villajero et al., 2010). More than 9 million people in the U.S., primarily concentrated in Texas, Florida, Washington, California, Oregon, and North Carolina, are migrant farm workers (Carroll et al., 2005; Coppel et al., 2001; Fitzgerald, Chakraborty, Shah, Khuder, \& Duggan, 2003; P.B. Organista, K.C. Organista, \& Soloff, 1998; Passel, Caps, \& Fix, 2004; Rhodes et al., 2010). The South Florida region has been severely 
affected by the HIV epidemic, with elevated risks for the infection being detected in the migrant worker community (Fernandez et al., 2009).

Migrant workers are disproportionately affected by HIV due to poverty (Albarran \& Nyamathi, 2011), social isolation (Muñoz-Laboy, Hirsch, \& Quispe-Lazaro, 2009), lack of access to health care (Fitzgerald, Chakraborty, Shah, Khuder, \& Duggan, 2003), and lack of knowledge (Rhodes et al., 2010; Villajero, 2003). Among migrant workers in the U.S., the prevalence of HIV infection, possibly as high as $13.5 \%$, is estimated to be 10 times the national rate (Organista et al., 1998; Organista et al., 2000; Organista et al., 2004).

The migrant workers involved in the studies presented are a mix of migrant workers and seasonal workers. The study's definition of migrant workers conformed to the Public Health Services Act (1944) definition. The Public Health Services Act states that migrant workers are those individuals who are employed in agricultural labor, either seasonal or migratory, and live in temporary housing. The study criteria did not limit the definition of migrant workers to those who travel 75 miles or cross county lines to work. The study also included some people who work or worked indirectly in agriculture, such as in packing houses. Even though the majority of migrant workers were farm workers, there were migrant workers with other types of employment.

The majority of migrant workers in the U.S. are originally from Mexico. The estimated number of Mexican migrants is reported, by the Centers for Disease Control and Prevention (CDC) (2009), to be as high as 12.7 million, and half of them are living here illegally or are undocumented. Hispanics account for about $70 \%$ of all U.S. migrant workers (Villajero et al., 2010). Some of the challenges that arise for migrant workers 
because of migration make them more vulnerable to sexually transmitted infections (STIs) in general, especially the HIV infection. Migrant workers are a highly mobile population which makes it challenging to properly monitor the incidence and prevalence rates of HIV in this vulnerable population (Villajero et al., 2010). The average income level of migrant workers tends to fall below the national poverty line (Villaregjo et al., 2010). Annually, single individuals make less than $\$ 7,500$ while five-person households make less than $\$ 10,000$ (Hansen \& Donohoe, 2003). On average, for migrant workers, the highest educational level reached is seventh grade, and $20 \%$ have been reported to be completely illiterate (Albarran \& Nyamathi, 2011; Denner, Organista, Dupree, \& Thrush, 2005; Hansen \& Donhoe, 2003). Little formal education and low income status contribute to the challenges that migrant workers face which make them more susceptible to negative health outcomes and HIV (Martinez-Donate et al., 2005; McCoy, Hlaing, Ergon-Rowe, Samuels, \& Malow, 2009).

Lack of access to health care services, lack of availability of health promotion programs, social isolation, language barriers, and constant mobility are contributing factors in making migrant workers such a vulnerable group. The lack of access to health care services is due to issues such as underreporting of medical conditions. One example is the underreporting of agricultural hazards among migrant workers because they fear loss of wages, and they also have different perceptions of health and illness due to cultural differences (Hansen \& Donohoe, 2003). The conditions of clean drinking water and facilities for migrant workers that provide amenities for hand washing and toilet usage are required by OSHA regulations for farms that have more than 11 workers. 
Ninety-five percent of farms have fewer than 11 workers which may allow farm owners not to comply with such regulations (Hansen \& Donohoe, 2003).

Social isolation has been a result of language barriers that migrants experience in their new country of residence. A coping mechanism used to deal with many hardships of their way of life is engaging in HIV risk behaviors (Hirsch, Higgins, Bentley, \& Nathanson, 2002). Muñoz-Laboy, Hirsch, \& Quispe-Lazaro's (2009) study on migrant workers in New York found that as levels of loneliness increased in participants, their number of unprotected vaginal acts with new girlfriends or sex workers increased. They also found that about $39 \%$ of their sample reported moderate levels of loneliness, and $35 \%$ reported high levels of loneliness. Eighty-three percent reported missing their family and lifestyle in Mexico, and 29\% reported not having anyone to talk to (Muñoz-Laboy, Hirsch, \& Quispe-Lazao, 2009). Participants reported that drinking alcohol, going to church, and interacting with friends were their outlets to cope with loneliness (MuñozLaboy, Hirsch, \& Quispe-Lazao, 2009).

One way migrant workers cope with the hardships of migration is through the consumption of alcohol and illegal substances. Participants from the study by MuñozLaboy et al. (2009) reported that alcohol consumption increased with every year a migrant worker is in the U.S. (Duke \& Gomez, \& Carpinetro, 2009; Winkleby et al., 2003). Worby \& Organista (2007) suggested that migrant workers' drinking levels were lower than U.S. Latinos or non-Latino populations in the U.S. A study by Worby and Organista (2007) suggested that there is a connection made between acculturative stress and alcohol consumption in migrant workers. The study conducted by Goodwin and Fox, which examined alcohol use and intimate-partner violence among migrant workers, 
concluded that the majority of the male migrant worker participants were at risk of being diagnosed for "lifetime alcohol abuse or dependency" (Kim-Godwin \& Fox, 2009, p. 138). These studies suggest that migrant workers tend to be at high risk for alcohol abuse and have been found to consume high levels of alcohol to cope with their stressful labor migration experiences.

Prior research suggests that a decline in attachment to one's ethnic group or ethnic identity is common among individuals who have arrived in a new country at a young age or have lived a long period of time in the new country. The generations most affected by this phenomenon are the first and the second born in the U.S. However, the members of the third generation, people who have lived in the U.S. all their lives, show no significant difference in ethnic identity or acculturation in comparison to their non-migrant counterparts (Garcia \& Lega, 1979; Phinney, 1990). This decline in identifying with one's ethnic group has been linked to higher levels of depressive symptoms and lower self-esteem as was found in a study by Umana-Taylor and Updegraff (2007) on Latino adolescents, primarily first generation born in the U.S.

In order to effectively reduce HIV/AIDS incidence and create effective prevention programs among this vulnerable group, there is a need to understand the contributory and protective factors to HIV risk behaviors. There is limited research on migrant workers, and although some research has been conducted on the impact of sexually transmitted infections (STIs) and HIV on migrant workers, none to date have examined ethnic identity as a protective factor on HIV risk behaviors. A deeper understanding of ethnic identification among migrant workers may assist in determining the factors important in enhancing health promotion programs. 


\section{Public Health Significance}

Most individuals affected by HIV/AIDS in the U.S. are from minority groups (Office of Minority Health, 2011; Centers for Disease Control and Prevention, 2010). According to the Office of Minority Health (OMH), a branch of the U.S. Department of Health and Human Services, in 2008, $71 \%$ of newly diagnosed HIV cases and $73 \%$ of newborns diagnosed with HIV/AIDS belong to minority groups (Centers for Disease Control and Prevention, 2010). The impact has been particularly high among African Americans and Hispanics (U.S. Department of Health \& Human Services, 2008; U.S. Department of Health \& Human Services, 2009; Office of Minority Health, 2011; Centers for Disease Control and Prevention, 2010). In 2009, the estimated rate of newly diagnosed HIV cases reported by the Centers for Disease Control and Prevention (CDC) was about 17.4 per $100,000,44 \%$ of those newly diagnosed cases were African American and 20\% were Hispanic (Centers for Disease Control and Prevention, 2011). African Americans and Hispanics accounted for 52\% and 17\% respectively of all HIV and AIDS cases diagnosed in 2008, even though they accounted for only $13 \%$ and $15 \%$ of the population, respectively (OMH, 2010). Latinos have the second highest HIV/AIDS rates in the country, resulting in about 25 cases per 100,000, with African Americans surpassing them at number one with 73.7 per 100,000 in 2008 (Centers for Disease Control and Prevention, 2010).

It is estimated that there may be over 9 million migrant workers in the U.S. The majority of migrant workers (approximately 70\%) are Hispanic (Fernandez, 2004; Passel, Capps, \& Fix, 2004; Worby and Organista, 2007). High levels of mobility and undocumented status explain why it is difficult to estimate exact population size of this 
subgroup. The same difficulty has been found in relation to HIV/AIDS prevalence and incidence rates among migrant workers. Migrant workers are affected by the HIV infection with rates reaching as high as 13.5\% (Gwyther, 1998; Hansen \& Donahoe, 2003; Weatherby et al., 1997). Research has suggested that migrant workers are at a heightened level of risk for HIV due to their engagement in a number of HIV risk behaviors, like risky sexual behaviors and alcohol and illegal substance use (Duke \& Carpintiero, 2009; Fernandez et al., 2004; Weatherby et al., 1997).

There are a number of challenges that migrant workers experience during their migration to a new place of residence which places them at a higher risk for negative health outcomes. Some of these challenges include constant mobility, poverty, lack of access to health care, low education levels, social isolation, and language barriers. Migrant workers' income tends to be below the poverty line, and in many cases, migrant workers endure unbearable working conditions (Hansen \& Donohoe, 2003). About 20\% of migrant workers have been reported to be illiterate, while the average grade level for Hispanic migrant workers is about seventh grade (Albarran \& Nyamathi, 2011). The constant mobility and uncertainty in relation to employment tends to increase the stress on migrant workers, which exacerbates the migration experience (Albarran \& Nyamathi, 2011; Hiott, Grzywacz, Davis, Quandt, \& Arcury, 2008). The lack of access to health care services leads to underreporting of medical conditions in the migrant worker community. Agricultural hazards tend to be underreported due to migrant workers' fear of loss of wages. Migrant workers may have different perceptions of health and illness due to cultural differences (Hansen \& Donohoe, 2003). 
A major problem affecting migrant workers is social isolation. Social isolation issues may be a result of language barriers and not knowing anyone in their new place of residence (Hirsch, Higgins, Bentley, \& Nathanson, 2002). Migrant workers often cope with social isolation through seeking comfort in sexual intimacy by engaging in unsafe sexual practices (Apostolopoulos et al., 2006; Arcury \& Quandt, 2007; Henry J. Kaiser Family Foundation (HJKFF), 2007; Organista et al., 2004; Parker, Easton, \& Klein, 2000). Such risky sexual behaviors put migrant workers, as well as their families, at high risk of STIs and the HIV infection. These risky sexual behaviors include unprotected vaginal sex and multiple sex partners. Similar findings were found in the study by Muñoz-Laboy, Hirsch, \& Quispe-Lazaro (2009) which concluded that risky sexual behaviors were associated with higher levels of loneliness in migrant workers in New York (Muñoz-Laboy, Hirsch, \& Quispe-Lazaro, 2009).

Alcohol and illegal substance abuse have also been identified as unhealthy coping mechanisms practiced among migrant workers (Hansen \& Donohoe, 2003; Weatherby et al., 1997). A study by Fernandez and her colleagues (2004) suggested that migrant workers who consumed marijuana or cocaine were at higher sexual risk for HIV transmission, which was indicated as a possible coping behavior among migrant workers (Fernandez et al., 2004).

Migrant workers are concentrated in six states in the U.S.; that is, Texas, Florida, Washington, California, Oregon, and North Carolina (Carroll et al., 2005; Rhodes et al., 2010). Elevated risks for HIV infection in the migrant worker community have been detected in the aforementioned six states (Fernandez et al., 2004). The migrant worker community suffers from poverty, lack of access to health care services, and low levels of 
education. A lack of government funded health programs makes it even more important to further study the HIV risk behaviors of this often overlooked migrant worker community. 


\section{Chapter II}

\section{LITERATURE REVIEW}

\section{HIV/AIDS Background and Public Health Impact}

HIV/AIDS has been spreading rapidly in the U.S., especially among minority groups. One of the subgroups which has been severely affected by the spread of HIV is migrant workers (Albarran \& Nyamathi, 2011; University of California, 2009). More than 9 million people in the U.S. are migrant workers, and $70 \%$ of migrant workers are of Hispanic ethnicity (Villajero et al., 2010). A large majority of migrant workers are located in six states in the U.S. These states are Texas, Florida, Washington, California, Oregon, and North Carolina (Carroll et al., 2005; Coppel et al., 2001; Fitzgerald, Chakraborty, Shah, Khuder, \& Duggan, 2003; P.B. Organista, K.C. Organista, \& Soloff, 1998; Passel, Caps, \& Fix, 2004; Rhodes et al., 2010).

The sample used in this study is located in South Florida. Florida has the third highest HIV/AIDS rate in the U.S., following New York and California (Lieb, 2006; Painter, 2008). Forty-six percent of newly diagnosed HIV cases and $40 \%$ of people living with HIV are located in the South; even though only $36 \%$ of the nation's population resides in the South (Painter, 2008). The South Florida region has been severely affected by the HIV epidemic, and elevated risks were detected in the migrant worker community located in South Florida (Fernandez et al., 2004).

A major factor that has contributed to the lack of research on migrant workers is that it is difficult to track migrant workers because they are constantly changing their place of residence. Migrant workers' coping mechanisms, due to their migration, heightens their level of risk for HIV transmission, particularly in the high risk states. It is 
therefore very important that efforts be made to investigate the factors which impact their risky behaviors as these factors would be important to include in HIV prevention services.

\section{Migrant Workers}

Migrant workers are an under studied group, in part because their mobility presents challenges to researchers who want to engage in long-term investigation. In the U.S., as mentioned previously, the majority of migrant workers originate from Spanish speaking countries. Many of these foreign born migrant workers have low levels of education and are unskilled (Hansen \& Donohoe, 2003; Villarejo, 2003; Villarejo et al., 2010). In the U.S., migrant workers support the economy by working in low wage service and production jobs because of, in many cases, their illegal immigration status (Passel, Capps, \& Fix, 2004; Worby \& Organista, 2007). The majority of migrant workers are young men who must spend long periods of time away from their homes (Denner, Organista, Dupree, and Thrush, 2005; Passel, Capps, \& Fix, 2004; Worby \& Organista, 2007).

There is limited research found on migrant workers, particularly regarding their HIV/AIDS risk. This marginalized subgroup of the population tends to be overlooked, even though they encounter innumerable hardships relating to their migration. Migrant workers' undocumented legal status and particularly mobile lifestyle contribute to the lack of health research conducted on this community. With these facts in mind, it is vital for public health professions to explore the needs of this underserved community and create effective interventions to better their health outcomes. 


\section{Migrant Workers and HIV Risk}

Migration is often associated with isolation, depression, and loneliness (Apostolopoulos et al., 2006; Arcury \& Quandt, 2007;Organista et al., 2004; HJKFF, 2007; Fernandez et al., 2004; Ford, King, Nerenberg, \& Rojo, 2001; P.B. Organista, K.C. Organista, Bola, Garcia de Alba, \& Moran, 2000). These factors, together with language difficulties and limited access to health care and prevention knowledge, limit the options that migrant workers have in terms of healthy coping strategies (Muñoz-Laboy, Hirsch, \& Quispe-Lazaro, 2009). Social isolation due to the pressures of migration is a major driving force for practicing unhealthy coping behaviors, such as seeking comfort in sexual intimacy, unsafe sexual practices, and drug use behaviors (Muñoz-Laboy, Hirsch, \& Quispe-Lazaro, 2009; Organista \& Kubo, 2005). These unsafe practices put migrant workers, as well as their families, at high risk of the HIV infection (Apostolopoulos et al., 2006; Arcury et al., 2007; HJKFF, 2007; Organista et al., 2004; Parker, Easton, \& Klein, 2000).

Low socioeconomic status, low formal education level, unstable employment, hazardous working conditions, and the stress of migration to a new environment are a few of the life circumstances that migrant workers endure. These life circumstances weaken their ability to attain more positive health outcomes and increase their exposure to HIV and sexually transmitted infections (STIs) (Bletzer \& Weatherby, 2009; Fernandez et al., 2004; Hirsch, \& Quispe-Lazaro, 2009; Mishra, Conner, \& Magana, 1996; Muñoz-Laboy, Hirsch, \& Quispe-Lazaro, 2009). These challenges have been linked to increased stress and unhealthy coping mechanisms such as increased alcohol 
consumption and risky sexual practices (Denner, Organista, Dupree, \& Thrush, 2005; Mishra, Conner, \& Magana, 1996; Muñoz-Laboy, Hirsch, \& Quispe-Lazaro, 2009). A connection has been made between migration and risky sexual behaviors in specific states in Mexico. Approximately $25 \%$ to $40 \%$ of all HIV/AIDS cases in rural areas in Mexico have been linked to Mexicans who have previously migrated to the U.S. (Rangel et al., 2005). The rural areas in Mexico that have high concentrations of migrants traveling to the U.S. account for approximately one-third of all AIDS cases in Mexico (Erlich, Organista, \& Oman, 2007; Rangel et al., 2005).

The length of stay in the U.S. after migration has been shown to increase HIV risk. Research among a sample of migrant workers of Latino origin, who had been in the U.S. for about 12 years, found high levels of injection drug use, males having sex with males (MSM), and sex with commercial sex workers, compared to more recent Latino migrant worker samples (Denner, Organista, Dupree, and Thrush, 2005). The factors contributing to higher levels of risky behaviors among the longer staying sample may be related to the factors found in a study conducted by Ehrlich, Organista, and Oman (2007). Their findings suggest that although a majority of the male migrants in their sample had primary partners back home, these migrant workers still reported being sexually active in the U.S. Approximately half of the sample was at high risk of HIV transmission due to risky sexual behaviors, such as unprotected sexual episodes with female partners (Erlich, Organista, \& Oman, 2007).

Several studies have examined the factors associated with higher risk of HIV transmission among migrant workers. For example, a study by Denner, Organista, Dupree, and Thrush (2005) explored psychosocial, behavioral, and demographic 
characteristics with HIV risk among marginally housed and migrant Latinos. The findings suggested being female, having lower levels of condom use self-efficacy, and being married were predictors for higher levels of HIV risk. HIV risk behaviors examined in this study included the number of sexual partners in the last two months, condom use, and risky sexual behaviors. Another study by Erlich, Organista, \& Oman (2007) found that migrant workers who exhibited lack of awareness about the level of HIV risk associated with risky sexual behaviors were least likely to intend to get tested for HIV. This study on migrant worker intentions to test for HIV emphasized the importance of education and knowledge in the reduction of HIV risk (Ehrlich, Organista, \& Oman, 2007).

Substance use has been found to play an important role in increasing HIV risk behaviors by causing lower inhibitions and diminished mental judgment (Duke \& Gomez, 2009; Organista \& Kubo, 2005). Alcohol consumption has also been associated with higher risk of STIs, including the HIV infection (Cook \& Clark, 2005). It is common among migrant workers to consume high levels of alcohol. The Muñoz-Laboy (2009) study on loneliness in migrant workers reported that migrant workers consumed alcohol to cope with their loneliness (Muñoz-Laboy, Hirsch, \& Quispe-Lazaro, 2009). It has been suggested that Hispanic migrant workers tend to consume more alcohol in one sitting than is the norm among drinkers in the U.S.; however, their frequency of drinking alcohol is less than the U.S. born drinkers (Medina-Mora, Borges, \& Villatoro, 2000; Worby \& Organista, 2007). A study by Organista and Kubo (2005) reported that a convenience sample of Hispanic day laborers reported that most of their sexual activities in the last two months involved alcohol consumption (Organista \& Kubo, 2005). This same study 
reported that the actual frequency of drinking was not very high, even though the number of drinks per drinking episode averaged seven (Organista \& Kubo, 2005). The drinking patterns reported suggest that alcohol consumption, in many cases, may lead to risky sexual behaviors which make it an HIV risk behavior.

Fernandez and colleagues (2004) have shown that migrant workers in South Florida have a high prevalence rate of STIs reaching about 16\% in a South Florida migrant worker sample (Fernandez et al., 2004). Over $50 \%$ of their sample did not use condoms, and those who engaged in substance use were over three times more likely to be at risk for HIV infection. Female migrant workers, in the same sample, were four times more likely to be at risk for HIV than male migrant workers (Fernandez et al., 2004). Sexual risk was measured by the number of partners in the last 12 months; if a condom was used; and the frequency of sexual encounters, vaginal or anal, in the last three months (Fernandez et al., 2004). It is apparent that migrant workers are at high risk for the HIV infection due to their unhealthy sexual practices. It is important to explore effective ways to reduce these unhealthy sexual practices in the migrant worker community. This study further explores HIV risky behaviors (which include risky sexual behaviors as well as the consumption of alcohol, crack, and marijuana) and explores its association with ethnic identity.

\section{Ethnic Identity and Acculturation}

In terms of health, studies have posited that ethnic identity may be associated with positive health behaviors like healthy lifestyle practices. Such healthy lifestyle practices include low consumption of alcohol, no/low substance use, and practicing HIV preventative behaviors (Brook, Whiteman, Balka, Win, \& Gursen, 1998; Nesdale, 
Rooney, \& Smith, 1997). Ethnic identity (EI), as defined by Phinney, DuPont, Espinosa, Revil, and Sanders (1994), is “a feeling of belonging to one's group, a clear understanding of the meaning of one's membership, positive attitudes toward the group, familiarity with its history and culture and involvement in its practices" (p.169). Ethnic identity includes aspects of ethnic involvement such as language, behavior, values, and knowledge of ethnic group history (Persky et al., 2005; Salgado de Snyder, Díaz Pérez, \& Maldonado, 2006). Phinney further defines the components of ethnic identity as ethnic identity exploration and ethnic identity belonging (Phinney, 1992). Ethnic identity "belonging", one of the components of ethnic identity included in Phinney's Multigroup Ethnic Identity Measure (MEIM) (one of the primary measures used in this study), contains ethnic pride, positive feelings toward one's ethnic group and one's satisfaction with being a member of his/her ethnic group. Ethnic identity "explore" is the second component. Ethnic identity explore measures the extent to which an individual searches for a sense of meaning in his/her ethnicity (Phinney, 1992). A moderating factor to changes in the development and exploration of ethnic identity in individuals is being a member of an ethnic minority group. In addition to being a minority, developmental levels of ethnic identity tend to be stronger in females than in males (Kiang, Witkow, Baldelomar, \& Fuligni, 2009).

Another concept commonly confused with ethnic identity, but a separate construct, is acculturation. For the purpose of this dissertation, it is very important to properly define acculturation and explain the difference between ethnic identity acculturation so no confusion between the constructs occurs. Acculturation has been defined as "how ethnic minority individuals adapt to the dominant culture and the 
associated changes in their beliefs, values, and behaviors that result from contact with the new culture" (Farver, Narang, \& Bhadha, 2002, p.338). Acculturation is usually thought to be associated with distancing from one's native roots. However, an interesting phenomenon is proposed by researchers Sanchez and Fernandez (1993) that higher acculturation to mainstream culture is not necessarily accompanied by the abandonment of the native culture. This type of bi-dimensional relationship changes the dynamic of acculturation theories. In essence, individuals' acceptance of the mainstream culture does not necessarily mean that they do not also identify with their own ethnic group. Sanchez and Fernandez (1993) concluded that high ethnic identification alone does not prevent or reduce acculturative stress. This stress seems to be reduced when there is a high acceptance of the dominant culture in the community in conjunction with high ethnic identification with the individual's ethnic group (Sanchez \& Fernandez, 1993).

Many studies use acculturation and ethnic identification as one construct, even though it is crucial to recognize their differences. Acculturation is associated with changes in attitudes or beliefs in relation to the mainstream or dominant culture. This concept is usually measured on a group level, while ethnic identification is assessed on the individual level. The majority of studies that measure acculturation fail to assess the multidimensional component of this construct (Abraido-Lanza et al., 2006). Ethnic identification measures the individual's emotional attachment (comfort level) and the engagement in cultural practices of his/her ethnic group (Phinney, 1992). Literature on acculturation is included in this proposal to compensate for the lack of literature on ethnic identification and its impact on HIV risk among migrant adults. 


\section{Ethnic Identity (EI)}

Ethnic identity (EI) research developed in an attempt to answer the question of whether ethnic identity positively or negatively affects individuals' behaviors. A primary factor of one's ethnic identity is his/her attachment and how well one connects with his/her culture. Culture plays a dominant role in an individual's life. It is vital to explore, therefore, how culture affects an individual's behaviors. This understanding of the interrelationship between culture and social behaviors may ultimately assist in reducing ethnic minorities' risk of and engagement in unhealthy behaviors.

Ethnic identity research began in children, specifically targeting the association between ethnic identity and academic achievement. Children were the first group studied because of the importance of studying their development of self-identity as well as ethnic identity (Phinney, 1992, 1997). In the early 1990s, it was discovered that adolescent children with a strong sense of ethnic identity had higher academic outcomes. The actual components of ethnic identity included a sense of belonging to one's ethnic group and the level of need to explore his/her cultural background (Taylor et al., 1994).

Ethnic identity has been shown to be positively associated with healthy psychosocial outcomes among ethnic minority college students (Espinosa \& Hernandez, 2009). These psychosocial outcomes, such as higher levels of self-efficacy and lower probability of depression, were associated with higher levels of ethnic identity. A study by Brook and Pahl (2005) demonstrated that as children got older, higher levels of ethnic identity during adolescent years served as a protective factor against drug use (Brook and Phal, 2005). 
The intriguing piece of ethnic identity is that the individual must perceive his/her ethnic group in a positive light in order to reap the benefits of membership in this ethnic group (Phinney, 1992; Phinney, Jacoby, \& Silva, 2007; Roberts et al., 1999). Increased barriers to academic, social, and career achievements have been suggested among individuals who have high ethnic identity but perceive their ethnic group in a negative manner (Phinney, Jacoby, \& Silva, 2007). This negative relationship between ethnic identity and achievement has been found among Latinos in relation to cigarette smoking (Castro, Stein, \& Bentler, 2009). The findings of the Castro, Stein, \& Bentler (2009) study showed that those who perceived their ethnic group in a negative way and felt attached to their ethnic group consumed more cigarettes than those with more positive perceptions of their ethnic group (Castro, Stein, \& Bentler, 2009). Studies have shown, for example, that higher ethnic identity among minority ethnic groups with stereotypes of high instances of alcohol consumption (example, Hispanic culture) and substance abuse tend to follow those stereotypes or unhealthy behaviors that are perceived as norms of that culture (Castro, Stein, \& Bentler, 2009; Smith, Walker, Fields, Brookins, \& Seay, 1999; Townsend \& Belgrave, 2000).

\section{Protective Role of Ethnic Identity}

Ethnic identity has been associated with less perceived discrimination, lower levels of psychological distress, and an overall better quality of life in minority individuals (Utsey, Chae, Brown, \& Kelly, 2002). Iturbide, Rafaelli, and Carlo (2009) examined the effects of ethnic identity on psychological well-being and found that Mexican American females who acquired higher ethnic identity also had lower levels of acculturative stress and depression; however, in males, ethnic identity exhibited mixed 
results. These mixed results suggested that male Mexican college students who experienced high levels of ethnic identity also had enhanced levels of self-esteem when they experienced low levels of acculturative stress. The same was not found, however, when the male Mexican student experienced a higher level of acculturative stress. These studies suggest the far-reaching impact of ethnic identity.

In terms of health, studies have posited that ethnic identity may be associated with positive behaviors, such as healthy lifestyle practices which may consist of low consumption of alcohol, no substance use, and practicing HIV prevention efforts (Brook, Whiteman, Balka, Win, \& Gursen, 1998; Nesdale, Rooney, \& Smith, 1997). Previous studies demonstrated that people living with HIV who had higher levels of ethnic identity not only had lower levels of psychological stress, but also found stronger social support systems and had higher levels of self-efficacy coping strategies (Crawford, Allison, Zamboni, \& Soto, 2002; Lopez, Antoni, Fekete, \& Penedo, 2010).

There have been some contradictory findings on the protective effects or the increase in risk related to ethnic identity's relationship with unhealthy/risky behaviors. A study conducted by Zamboanga and his colleagues found that higher levels of ethnic identity among adolescent Hispanics were associated with increased alcohol and cigarette use, but decreased marijuana use (Zamboanga, 2009). On the other hand, other studies have shown that adolescent immigrants with higher levels of ethnic identity engage in less substance abuse than their native-born counterparts (Marsiglia, 2008). A study conducted on urban African American girls by Corneille and Belgrave (2007), indicated that higher levels of ethnic identity were associated with higher levels of sexual refusal efficacy and disapproval of substance use (Corneille and Belgrave, 2007). This study 
demonstrates that ethnic identity has a positive effect on health behaviors and attitudes towards risky sexual behaviors. The mixed findings of these studies demonstrate how perception of one's ethnic group affects the influence of ethnic identity. If individuals view their ethnic group in a negative light, higher ethnic identity will encourage unhealthy behaviors, and the opposite is true as well.

Due to the limited literature on ethnic identity and its relationship with risky sexual behaviors and substance use, acculturation literature was also included to provide a clearer view on individuals who become more comfortable with the mainstream culture and, in some cases, lower their level of attachment to their ethnic group or have a low ethnic identity. Higher levels of acculturation have been associated with higher educational attainment, access to health insurance, and higher socioeconomic status among Latino migrants (Sanchez, Rice, Stein, Milburn, \& Rothman-Borus, 2009; Zambrana, Scrimshaw, Collins, \& Dunkel-Schetter, 1997). Further, ethnic minority HIV positive individuals who are more acculturated tend to take more proactive steps to improve their health, utilizing positive coping strategies related to accessibility of education and credible health information (Bianchi, Zea, Poppen, Reisen, \& Echeverry, 2004).

An issue with the literature on ethnic identity and acculturation is that there has not been one standardized tool for measurement used. Some studies use language or generational status to represent acculturation or the ethnic identity measure, instead of utilizing a measure which consists of multiple items (Abraido-Lanza, Armbrister, Florez, Aguirre, 2006). In this dissertation, a measurement tool to assess ethnic identity, the MEIM, will be used to limit such problems (Phinney, 1992). 


\section{Ethnic Identity and Changes over Time}

Since this dissertation also explores the changes in ethnic identity and its impact on HIV risk behaviors, it is important to review the limited literature on ethnic identity change and what triggers such changes. The literature suggests that ethnic identity changes over time, depending on the stage of development of an individual and changing life circumstances (Phinney, 1992, 2003). A study administered by Kiang, Witkow, Baldelomar, and Fuligni (2010) focused on ethnic identity change in high school students over a four-year period. This study revealed that increases in ethnic identity were associated with the level of closeness these high school students felt toward their parents and their same ethnic peer relationships. Increases in ethnic identity were found in students whose ethnic group was not a majority in the school. Unfortunately, this study did not assess if such changes coincided with increases in positive outcomes such as academic achievement (Kiang, Witkow, Baldemar, \& Fuligni, 2010).

Investigating changes in ethnic identity is not common; however, there have been a limited number of studies that explored ethnic identity changes in adulthood. Those studies concluded that changes in ethnic identity can occur over time (Phinney, 2007; Torres et al., 2010). For example, a study by Torres and colleagues (2010) found that ethnic identity does change in Latinos during adulthood due to changes in the environment. New encounters and life altering obstacles may bring about a developmental change in one's identity which will affect ethnic identity. This change in ethnic identity was named "identity reconstruction" by Marcia (1966, p.15). A change in ethnic identity revolves around reevaluating one's identity and attachment to one's ethnic group. Among adults, therefore, it seems that instability will ultimately lead to changes in 
ethnic identity. Ethnic identity change has not been examined for associations with positive or negative health outcomes (Phinney, 2007; Torres et al., 2010).

\section{Ethnic Identity and HIV Risk Behaviors}

\section{Risky Sexual Behaviors}

Although the literature on the relationship between ethnic identity and risky sexual behaviors is sparse, limited numbers of studies have been conducted among various ethnic groups. Some of the studies present a theory of ethnic identity as a protector against taking on risky sexual and substance use behaviors. For example, in a study conducted by Beadnell and colleagues (2003), a "buffering” effect was shown among African American heterosexual females by strength of ethnic identity. A difference in the number of risky sexual acts in the last four months was found between those with higher levels of ethnic identity compared to those with lower ethnic identity levels.

A study by Espinosa-Hernandez and Lefkowitz (2009) suggested that ethnic identity also affects the attitudes and beliefs of individuals. These researchers conducted a study in an ethnic minority college student sample which examined the association of risky sexual behaviors and attitudes with ethnic identity. The study suggested that a higher level of ethnic identity commitment/belonging, a subscale of Multigroup Ethnic Identity Measure (MEIM), served as a protective factor with risky sexual behaviors and risky sexual attitudes among European American college students (Espinosa-Hernandez \& Lefkowitz, 2009).

Sexual attitudes of urban African American adolescent females were explored by Corneille and Belgrave (2007). This study demonstrated that higher levels of ethnic 
identity were linked to lower levels of risky sexual attitudes, such as "higher sexual refusal efficacy, higher disapproval to use drugs, and lower intentions to use" drugs (Corneille \& Belgrave, 2007, abstract). A study by Belgrave, Van Oss Marin, \& Chambers (2000), targeted African American girls between the ages of 10 and 13 years of age, and examined the impact of ethnic identity and self-esteem on risky sexual attitudes. The study found that age was a strong predictor of risky sexual attitudes, demonstrating that older girls had more risky sexual attitudes. The Belgrave et al. study also suggested that lower levels of ethnic identity may be connected with higher levels of alienation which ultimately may lead to more negative coping mechanisms, such as engaging in high risk behaviors (Belgrave, Marin, \& Chambers, 2000; Brook \& Pahl, 2005; Cislo, 2008, Smith, Walker, Fields, Brookins, and Seay, 1999; Phinney, 1997; Umana-Taylor, Gonzales-Backen, \& Guimond, 2009).

Literature on acculturation is included in this section to shed some light on sexual behaviors of individuals who are more comfortable with mainstream culture compared to those who are attached to their ethnic group. In the U.S., various levels of acculturation have been associated with both positive and negative health outcomes related to HIV/AIDS risk, particularly among Hispanics. A study by Bianchi, Zea, Poppen, Reisen \& Echeverry (2004) found that lower levels of acculturation may be a protective factor for individuals relating to health outcomes, such as lower levels of depression, less substance abuse, and risky sexual behaviors (Bianchi, Zea, Poppen, Reisen, \& Echeverry, 2004). This beneficial effect may differ depending on the circumstances and situation of the individual (Schwartz, Zamboanga, \& Jarvis, 2007). Another study by Newcomb and Carmona (2004) showed that Hispanic females who are highly acculturated tend to have 
more permissive views relating to sexual behaviors, number of sexual partners, and unhealthy modes of coping, such as drug use (Newcomb \& Carmona, 2004).

With regard to migrant workers, studies suggest that those who have lived in the U.S. longer are more acculturated to the English language, American lifestyle, and tend to have increased HIV risk behaviors compared to those who are less acculturated (Hussey, Hallfors, Waller, Iritani, Halpern, \& Bauer, 2007; Kasirye, Walsh, Romano, Beckett, Garcia, Elvine-Kreis, Bethel, \& Schenker, 2005; Marks, Cantero, \& Simoni, 1998; Salgado de Snyder et al., 1996).

Alcohol and Substance Use

Alcohol and drug use, and their relationship to ethnic identity, have been minimally studied. The altered mental status linked to alcohol and drug use has been associated with risky sexual behaviors, such as unprotected sexual acts (Cooper, 2002; Noriis, Masters, \& Zawacki, 2004; Seth, Wingood, DiClemente, \& Robinson, 2011). Many articles have shown the link between drug abuse and risky sexual practices (Brown \& Van Hook, 2006; Duncan, Boisjdy, Kremer, Levy, Eccles, 2005). However, studies addressing alcohol consumption at non-abuse levels are almost non-existent. In addition, literature on the association between substance use and ethnic identity is scarce.

The studies on ethnic identity and alcohol have shown that an inverse relationship seems to exist between ethnic identity and alcohol and/or drug use. For example, higher levels of ethnic identity were associated with lower levels of substance use (Beadnell et al., 2003; Townsend and Belgrave, 2000). A study by Townsend and Belgrave (2000) examined racial identity in African American children. Children's Black Identity Scale was used to examine racial identity which measures how closely the child identities with 
his/her racial group. The results suggested that higher levels of racial and personal identity were negatively associated with drug use and attitudes towards drug use. Similar to ethnic identity, higher levels of racial identity were associated with lower levels of drug use. The study conducted by Beadnell et al. (2003) examined the extent that baseline levels of ethnic identity offset negative psycho-behavioral characteristics in African American adolescents, ultimately affecting their substance abuse practices in a positive manner (Beadnell et al., 2003). This study also explored how ethnic identity would affect outcomes in an HIV risk reduction intervention, and ultimately those with higher ethnic identity at baseline had more positive outcomes in risky sexual behaviors over a four month period compared to participants with low ethnic identity (Beadnell et al., 2003).

Brook and Pahl (2005) examined ethnic identity and drug use in an adult African American sample. They concluded that the higher the attachment to the ethnic group or the higher the level of ethnic pride (same as ethnic identity), the lower the level of drug use and symptoms of depression. Similar findings were found by Brook and colleagues (1998) in Hispanic samples. Their study suggested that ethnic identity had a protective effect among Hispanic adolescents. Higher ethnic identity was associated with negative attitudes toward drug use and the adolescents with higher ethnic identity were less likely to engage in drug use behaviors.

There seems to be some inconsistency in the results of studies assessing alcohol use and ethnic identity. It is mentioned in some of the studies which target Hispanics that a positive relationship seems to exist between ethnic identity and alcohol consumption, and that may be because alcohol consumption seems to be encouraged in the Hispanic culture, especially among males. There have been studies that have illustrated that 
Hispanic males may have high levels of alcohol consumption if they have a high level of ethnic identity (Albarran \& Nyamathi, 2011; Worby \& Organista, 2007). This finding may be due to the cultural norms on alcohol consumption. It has been suggested that the stereotype of Hispanic males is that they heavily consume alcohol. The norm may be in opposition to the protective role of ethnic identity since in this case, higher levels of ethnic identity coincide with cultural norms (Zamboanga, Rafaelli, \& Horton, 2006).

\section{Moderating Variables on Ethnic Identity and HIV Risk}

Studies have been conducted in an attempt to understand the factors that influence the impact of ethnic identity. There are studies that have found social support to be associated with ethnic identity and one study has shown that they moderate ethnic identity (Lopez, Antoni, Fekete, \& Penedo, 2010). For the purposes of this dissertation, social support is assessed for moderating effects and will be discussed.

\section{Social Support}

A major factor studied for decades in relation to health outcomes has been social support. Social support can be defined in a number of ways. Structurally, it can be described on the basis of social integration, which states an individual is part of a network and socially participates in that network (Reblin \& Uchino, 2008). The other aspect of social support involves the functional element, which embodies actual social transactions between people. This social interaction constitutes emotional support (Piferi \& Lawler, 2006; Reblin \& Uchino, 2008).

Social support has been consistently linked with lower levels of mortality and morbidity (Cole, Matheson, \& Anismen, 2007; Lee and Rotheram-Borus, 2001; Lyyra \& Heikkinen, 2006; Rutledge et al., 2004). The lower levels of mortality and morbidity may 
be an effect of social support, which may be labeled as a health promoting element. Social support may influence an individual's health behaviors and practices, such as cigarette smoking and alcohol consumption (Uchino, 2006). In the literature, social support has been repeatedly associated with more positive health outcomes, such as higher infant birth weight (Campos et al., 2008; Dunkel-Schetter, Gurung, Lobel, \& Wadhwa, 2000; Uchino, 2006). It has also been suggested that social support provides a buffer against stressful health conditions which indirectly affect health outcomes.

Examples of how social support indirectly affects health outcomes include stress induced cardiovascular health (e.g., lower blood pressure) and lower levels of atherosclerosis (Dunkel-Schetter, Gurung, Lobel, \& Wadhwa, 2000; Sagrestano, Feldman, Killingsworth-Rini, Woo, \& Dunkel-Schetter, 1999; Uchino, 2006). Social Support and Ethnic Identity

Previous studies have shown that higher levels of ethnic identity were associated with stronger social support. Individuals with higher ethnic identity also had an increased likelihood of utilizing social support services (Lopez, Antonio, Fekete, Penedo, 2010; Yoo \& Lee, 2005). The studies exploring social support and ethnic identity are based on minority group samples; however, none of the studies examine social support and ethnic identity in a migrant worker sample. The closeness and level of belonging individuals feel with their ethnic group may encourage an increased level of social support from members of their ethnic group. Both factors may contribute to more positive outcomes in relation to health and academic achievement (Noh \& Kaspar, 2003; Phinney, Horenczyk, Liebkind, \& Vedder, 2000). A study by Cole and her colleagues (2007) suggested that ethnic identity and social support are moderating factors in the mental well-being and 
academic performance of adolescents from ethnic minorities. Another study by Lopez and her colleagues (2010) concluded that women living with HIV who acquired high levels of ethnic identity had less perceived stress. This was suggested to be a result of better coping self-efficacy and access to more social support resources (Lopez, Antoni, Fekete, \& Penedo, 2010). These studies describe how social support and ethnic identity seem to provide a protective shield against negative health outcomes and academic failure.

\section{Conclusion}

The high rates of HIV/AIDS among migrant workers, as high as $13 \%$ by some reports, illustrate the hardships that frequently accompany the lives of migrant workers (Gwyther, 1998; Hansen \& Donahoe, 2003; Weatherby et al., 1997). These hardships, language barriers, limited access to health care, little education, and low wages, are often accompanied by feelings of depression, isolation, and loneliness. These feelings often propel members of this group to engage in unsafe behaviors which ultimately increase their vulnerability to HIV. It is important, therefore, to find effective strategies to encourage the practice of healthy behaviors.

Previous research by Phinney $(1992,2003$; Torres, 2010) has found that unstable stages or traumatic experiences in a person's lifespan may lead individuals to question their sense of identity. Migrants often find themselves in unstable situations due to poverty, continuous movement, language difficulties, and because many of them are ethnically different from the majority population. Therefore, they might question their sense of ethnic identity. Research has found that one's ethnic identity, as a part of one's self-identity, may play a protective role regarding healthy lifestyle behaviors, for 
example, risky sexual behaviors and substance use. Such a protective role has been documented in college students as well as adolescents (Cornell \& Belgrave, 2007; Espinosa-Hernandez \& Lefkowtiz, 2009). If this same protection is found among migrant workers, then a strong sense of ethnic identity may be associated with a reduction in high HIV risk behaviors among vulnerable, underserved migrants and ultimately lead to a reduction in the spread of HIV. The following studies explore the relationship between ethnic identity and HIV risk behaviors and examine the effects of short-term changes in ethnic identity on changes in HIV risk behaviors and alcohol expectancy measures.

\section{Research Questions}

The fundamental questions guiding this research are as follows:

1. Research conducted on ethnic identity has illustrated that it may have a protective effect on engagement in negative health and risky behaviors in younger adults. Does ethnic identity have a positive effect on HIV risk behaviors in migrant workers, reducing their chances of transmitting HIV? Does such a relationship exist between ethnic identity and risky sexual behaviors among migrant workers that have been sexually active in the last three months?

2. Does ethnic identity change over time in adult migrant workers? If ethnic identity does change, does ethnic identity change still influence HIV risk behaviors in a protective manner or does it remain constant? Are there demographic factors that may be associated with positive or negative changes in HIV risk behaviors?

3. One of the intervention groups utilized in the parent study, PEER, provided ethnically sensitive scenarios to its' African American and Hispanic participants. Did assignment into this specific intervention possibly increase the ethnic identity 
of the participants since more ethnically based material was provided and participants of the same ethnicity participated as peers to new incoming participants? The literature suggests that patients in treatment centers that are ethnically matched with health care providers tend to have more positive treatment results than patients that are not ethnically matched. Does ethnic identity increase in groups with the same ethnic peers in comparison with groups that are more varied?

Research Question 1: Is ethnic identity associated with HIV risk behaviors at baseline? This will be examined through assessing the relationship between ethnic identity and self-reported HIV risk behaviors at baseline among migrant workers.

Hypothesis 1a: Higher ethnic identity will be inversely associated with risky sexual behaviors at baseline.

Hypothesis $1 b$ : Higher levels of ethnic identity will be inversely associated with levels of alcohol use at baseline.

Hypothesis 1c: Higher levels of ethnic identity will be inversely associated with drug use at baseline.

Hypothesis 1 : Higher levels of ethnic identity will be inversely related to total Sex Related Alcohol Expectancies (SRAE) score and the subscales for SRAE (SRAE-Enhanced Sex, SRAE- Decreased Nervousness, and SRAE-Increased Risk) at baseline.

Research Question 2: Does ethnic identity change over six months, and if so, is it associated with changes in high-risk HIV behaviors? 
Hypothesis 2a: There will be differences in levels of ethnic identity between baseline and the 6-month follow-up period.

Hypothesis 2b: Ethnic identity scores at follow-up will be higher than ethnic identity scores at baseline.

Hypothesis 2c: Short-term changes in ethnic identity will be associated with changes in HIV risk behaviors.

Hypothesis $2 d$ : Increases in level of ethnic identity, from baseline to follow up, will be associated with decreases in sexual risk behaviors.

Hypothesis 2e: Increases in level of ethnic identity, from baseline to follow up, will be negatively associated with frequency of alcohol use.

Hypothesis 2f: Increases in level of ethnic identity, from baseline to follow up, will be negatively associated with frequency of drug use.

Hypothesis 2g: Increases in level of ethnic identity, from baseline to follow up, will be associated with decreases in Sex Related Alcohol Expectancies scores.

Research Question 3: Are changes in social support associated with changes in ethnic identity?

Hypothesis $3 a$ : Increases in levels of social support will be associated with positive changes in ethnic identity levels.

Hypothesis $3 b$ : Social support will moderate positive changes in ethnic identity scores in relation to changes in HIV risk behaviors and alcohol expectancies. 
Research Question 4: Are there differences in short term ethnic identity change when comparing assignment to the two community-based interventions (PEER vs. HEALTHY).

Hypothesis 4a: There will be a significant difference in short-term ethnic identity scores between the two community-based interventions.

Hypothesis $4 b$ : Ethnic identity change scores will be higher for subjects in the peer-assisted condition (PEER) intervention than for the health promotion condition (HEALTHY). 


\section{REFERENCES}

Abraido-Lanza, A. F., Armbrister, A. N., Florez, K. R., Aguirre, A. N. (2006). Toward a theory driven model of acculturation in public health research. American Journal of Public Health, 96(8), 1342-6. doi:10.2105/AJPH.2005.064980

Albarran, C. R., \& Nyamathi, A. (2011). HIV and Mexican migrant workers in the United States: A review applying the vulnerable populations conceptual model. The Journal of the Association of Nurses in AIDS Care: JANAC, 22(3), 173-85. doi:10.1016/j.jana.2010.08.001

Apostolopoulos, Y., Sonmez, S., Kronenfeld, J., Castillo, E., McLendon, L., \& Smith, D. (2006). STI/HIV risks for Mexican migrant laborers: Exploratory ethnographies. Journal of Immigrant and Minority Health / Center for Minority Public Health, 8(3), 291-302. doi:10.1007/s10903-006-9334-2

Arcury, T. A, \& Quandt, S. A. (2007). Delivery of health services to migrant and seasonal farmworkers. Annual Review of Public Health, 28, 345-63.

doi:10.1146/annurev.publhealth.27.021405.102106

Bandura, A. (1982). Self-efficacy mechanism in human agency. American Psychologist, $37,122-147$.

Beadnell, B., Stielstra, S., Baker, S., Morrison, D. M., Knox, K., Gutierrez, L., \& Doyle, A. (2003). Ethnic identity and sexual risk-taking among African-American women enrolled in an HIV/STD prevention intervention. Psychology, Health \& Medicine, 8(2), 187-198. doi:10.1080/1354850031000087564

Belgrave, F. Z., Marin, B. V. O., \& Chambers, D. B. (2000). Cultural, Contextual, and Intrapersonal Predictors of Risky Sexual Attitudes among Urban African American Girls in Early Adolescence. Cultural Diversity and Ethnic Minority Psychology, 6(3), 309-322. doi:10.1037//1099-9809.6.3.309

Bianchi, F. T., Zea, M. C., Poppin, P. J., Reisen, C. A., \& Echeverry, J. J. (2004). Coping as a mediator of the impact of sociocultural factors on health behavior among HIVpositive Latino gay men. Psychology and Health, 19, 89-101.

doi:10.1080/08870440410001655340

Brook, J. \& Pahl, K. (2005). The protective role of ethnic and racial identity and aspects of an Africentric orientation against drug use among African American young adults. Journal of Genetics, 166(3), 329-345. Retrieved from http:/www.ncbi.nlm.nih.gov/pmc/articles/PMC1315285/

Brook, J. S., Whiteman, M., Balka, E. N., Gursen, M. D., \& Win, P. T. (1998). Drug use among Puerto Ricans: Ethnic identity as a protective factor. Hispanic Journal of Behavioral Sciences, 20(2), 241-254. doi:10.1177/07399863980202007 
Brown, E. J., \& Van Hook, M. (2006). Risk behavior, perceptions of HIV risk, and risk reduction behavior among a small group of rural African American women who use drugs. Journal of the Association of Nurses in AIDS Care, 17, 42-50.

doi:10.1016/j.jana.2006.07.004

Carroll, D. J, Samardick, R., Bernard, S., Gabbard S., Hernandez, T. (2005). Findings from the National Agricultural Workers Survey (NAWS) 2001-2001: A demographics and employment profile of United States farmworkers. (Research Report No. 9, U.S. Department of Labor).

Castro, F. G., Stein, J. A., \& Bentler, P. M. (2009). Ethnic pride, traditional family values, and acculturation in early cigarette and alcohol use among Latino adolescents. The Journal of Primary Prevention, 30(3-4), 265-92. doi:10.1007/s10935-009-0174-z

Centers for Disease Control and Prevention (CDC). (2010). HIV Surveillance Report, 2008. Retrieved from:

http://www.cdc.gov/hiv/surveillance/resources/reports/2008report/pdf/2008SurveillanceR eport.pdf

Centers for Disease Control and Prevention (CDC). (2011). HIV in the United States: An overview. Retrieved from:

http://www.cdc.gov/hiv/topics/surveillance/resources/factsheets/pdf/HIV-USoverview.pdf

Cook, R. L., \& Clark, D. B. (2005). Is there an association between alcohol consumption and sexually transmitted diseases? A systematic review. Sexually Transmitted Diseases, 32(3), 156-164. doi:10.1097/01.olq.0000151418.03899.97

Corneille, M. A, \& Belgrave, F. Z. (2007). Ethnic identity, neighborhood risk, and adolescent drug and sex attitudes and refusal efficacy: the urban African American girls' experience. Journal of Drug Education, 37(2), 177-90. Retrieved from http://www.ncbi.nlm.nih.gov/pubmed/17977240

Crawford, I., Allison, K. W., Zamboni, B. D., \& Soto, T. (2002). The influence of dualidentity development on the psychosocial functioning of African-American gay and bisexual men. The Journal of Sex Research, 39(3), 179-189.

Denner, J., Organista, K. C., Dupree, J. D., \& Thrush, G. (2005). Predictors of HIV transmission among migrant and marginally moused Latinos. AIDS and Behavior, 9(2), 201-210. doi:10.1007/s10461-005-3901-3

Duke, M. R. \& Carpinteiro, F. J. G. (2009). The effects of problem drinking and sexual risk among Mexican migrant workers on their community of origin. Human Organization, 68(3), 328-344. doi:10.1016/j.bbi.2008.05.010 
Duncan, G. J., Boisjdy, J., Kremer, M., Levy, D. M., \& Eccles, J. (2005). Peer effects in drug use and sex among college students. Journal of Abnormal Child Psychology, 33(3), 375-385. doi:10.1007/s10802-005-3576-2

Ehrlich, S. F., Organista, K. C., \& Oman, D. (2007). Migrant Latino day Llaborers and intentions to test for HIV. AIDS Behavior, 11, 743-752. doi:10.1007/s10461-006-9182-7

Espinosa-Hernandez, G. \& Lefkowitz, E. S. (2009). Sexual behaviors and attitudes and ethnic identity during college. Journal of Sex Research, 46(5), 471-482.

doi:10.1080/00224490902829616

Farver, J. A. M., Narang, S. K., \& Bhadha, B. R. (2002). East meets West: Ethnic identity, acculturation, and conflict in Asian Indian families. Journal of Family Psychology, 16(3), 338-350. doi:10.1037//0893-3200.16.3.338

Fernandez, M. I., Collazo, J. B., Hernandez, N., Bowen, G. S., Varga, L. M., Vila, K. C., Arheart, K. L., \& Perrino, T. (2004). Predictors of HIV risk among Hispanic farm workers in South Florida: Women are at higher risk than men. AIDS and Behavior, $8(2)$, 165-173. doi:10.1023/B:AIBE.0000030247.00140.62

Field, C. \& Caetano, R. (2010). The role of ethnic matching between patient and provider on the effectiveness of brief alcohol interventions with Hispanics. Alcoholism: Clinical and Experimental Research, 34(2), 262-271. doi:10.1111/j.1530-0277.2009.01089.x

Ford, K., King, G., Nerenberg, L., \& Rojo, C. (2001). AIDS knowledge and risk behaviors among Midwest migrant farmworkers. AIDS Education and Prevention, 13(6), 551-560. doi: 10.1521/aeap.13.6.551.21432

Gwyther, E. (1998). Migrant farmworker children: Health status, barriers to care, and nursing innovations in health care delivery. Journal of Pediatric Health Care, 12(2), 6066. doi:10.1016/S0891-5245(98)90223-1

Hansen, E \& Donohoe, M. (2003). Health Issues of Migrant and Seasonal Farmworkers. Journal of Health Care for the Poor and Underserved, 14(2), 153-164. doi:10.1177/1049208903251513

Henry J. Kaiser Family Foundation. (2007). Migrant workers contributing to spread of HIV in rural Mexican states, researchers say. Retrieved from http://www.thebody.com/content/world/art42324.html

Hiott, A. E., Gryzywacz, J. G., Davis, S. W., Quandt, S. A., \& Arcury, T. A. (2008). Migrant farmworker stress: mental health implications. The Journal of Rural Health, 24(1), 32-39. doi:10.1111/j.1748-0361.2008.00134. 
Hirsch, S., Higgins, J., Bentley, M. E., \& Nathanson, C. A. (2002). The social constructions of sexuality: marital infidelity and sexually transmitted disease-HIV risk in a Mexican migrant community. American Journal of Public Health, 92(8), 1227-1237. doi: 10.2105/AJPH.92.8.1227

Hussey, J. M., Hallfors, D. D., Waller, M. W., Iritani, B. J., Halpern, C. T, Bauer, D. J. (2007). Sexual behavior and drug use among Asian and Latino adolescents: Association with immigrant status. Journal of Immigrant \& Minority Health, 9(2), 85-94.

doi: 10.1007/s10903-006-9020-z

Iturbide, M. I., Rafaelli, M., \& Carlo, G. (2009). Protective effects of ethnic identity on Mexican American college students' psychological well-being. Hispanic Journal of Behavioral Sciences, 31(4), 536-552. doi:10.1177/0739986309345992

Kalichman, S. C., Simbayi, L., Jooste, S., Vermaak, R., \& Cain, D. (2008). Sensation seeking and alcohol use predict HIV transmission risks: Prospective study of sexually transmitted infection clinic patients, Cape Town, South Africa. Addictive Behaviors, 33, 1630-1633. doi:10.1016/j.addbeh.2007.01.026

Kasirye, O. C., Walsh, J. A., Romano, P. S., Beckett, L. A., \& Garcia, J. A., Elvine-Kreis, B., Bethel, J. W., Schenker, M. B. (2005). Acculturation and its association with healthrisk behaviors in a rural Latina population. Ethnicity \& Dissease, 15(4), 733-9.

PMID:16259501

Kerpelman, J. L., Eryigit, S., \& Stephens, C. J. (2007). African American adolescents' future education orientation: Associations with self-efficacy, ethnic identity, and perceived parental support. Journal of Youth and Adolescence, 37(8), 997-1008. doi:10.1007/s10964-007-9201-7

Kiang, L., Witkow, M. R., Baldelomar, O. A, \& Fuligni, A. J. (2010). Change in ethnic identity across the high school years among adolescents with Latin American, Asian, and European backgrounds. Journal of Youth and Adolescence, 39(6), 683-93.

doi:10.1007/s 10964-009-9429-5

Lieb, S. (2006). Coalition to reduce HIV in Broward minority community. Bureau of HIV/AIDS Florida Department of Health. Retrieved from http://chua2.fiu.edu/faculty/darroww/reach2010/hiv_stats.htm

Lopez, C. R., Antoni, M. H., Fekete, E. M., \& Penedo, F. J. (2010). Ethnic identity and perceived stress in HIV+ minority women: The role of coping self-efficacy and social support. International Journal of Behavioral Medicine. doi: 10.1007/s12529-010-9121

Lowe, D. (1960) Harvest of Shame. CBS News with Edward Murrow. Retrieved from: http://www.cbsnews.com/video/watch/?id=7087479n\&tag=contentMain;contentBody. 
Marcia, J. E. (1966). Development and validation of ego identity status. Journal of Personality and Social Psychology, 3(5), 551-558. doi:10.1037/h0023281

Marsiglia, F., F. (2008). Immigrant advantage?Substance use among Latin American immigrant and native-born youth in Spain. Ethnicity \& Health, 13(2), 149-170. doi: $10.1080 / 13557850701830356$

Martínez-Donate, A. P., Rangel, M. G., Hovell, M. F., Santibáñez, J., Sipan, C. L., Izazola, J. A. (2005). HIV infection in mobile populations: the case of Mexican migrants to the United States. Revista Panamerican Salud Publica, 17(1), 26-9.

McCoy, H. V., Hlaing, W. M., Ergon-Rowe, E., Samuels, D., \& Malow, R. (2009). Lessons from the fields: A migrant HIV prevention project. Public health reports, 124(6), 790-6. Retrieved from http://www.ncbi.nlm.nih.gov/pmc/articles/PMC2773941/

Medina-Mora, M. E., Borges, G., \& Villatoro, J. (2000). The measurement of drinking patterns and consequences in Mexico. Journal of Substance Abuse, 12(1-2), 183-196.

Mishra S., Conner R., \& Magaña R. (1996). Migrant workers in the United States: a profile from the fields. In: S. Mishra, R. Conner, \& R. Magaña, (Ed.), AIDS Crossing Borders: The Spread of HIV among Migrant Latinos. (pp. 3-25). Boulder, CO: Westview Press

Mossakowski, K. N. (2003). Coping with perceived discrimination: Does ethnic identity protect mental health? Journal of Health and Social Behavior, 44(3), 318-31. Retrieved from http://www.ncbi.nlm.nih.gov/pubmed/14582311

Muñoz-Laboy, M., Hirsch, J. S., \& Quispe-Lazaro, A. (2009). Loneliness as a sexual risk factor for male Mexican migrant workers. American Journal of Public Health, 99(5), 802-810. doi: 10.2105/AJPH.2007.122283

Nesdale, D., Rooney, R., \& Smith, L. (1997). Migrant ethnic identity and psychological Distress. Journal of Cross-Cultural Psychology, 28, 569-588.

doi: $10.1177 / 0022022197285004$

Newcomb, M.D., \& Carmona, J.V. (2004). Adult trauma and HIV status among Latinas: Effects upon psychological adjustment and substance use. AIDS and Behavior, 8, 417428. doi: 10.1007/s10461-004-7326-1

Office of Minority Health (OMH). (2011, January 14). HIV/AIDS Data/Statistics. Retrieved from: http://minorityhealth.hhs.gov/templates/browse.aspx?lvl=3\&lvlid=70

Ojeda, L., Pina-Watson, B., Castillo, L. G., Castillo, R., Khan, N., \& Leigh, J. (2011). Acculturation, enculturation, ethnic identity, and conscientiousness as predictors of Latino boys' and girls' career decision self-efficacy. Journal of Career Development. doi: $10.1177 / 0894845311405321$ 
Organista P. B., Organista K. C., \& Soloff, P. R. (1998). Exploring AIDS related knowledge, attitudes, and behaviors of female migrant workers. Health and Social Work. 23(2):96-103.

Organista, K. C., Kubo, A. (2005). Pilot survey of HIV risk and contextual problems and issues in Mexican/Latino migrant day laborers. Journal of Immigrant Health, 7(4), 269281.

Organista, K. C. \& Organista, P. B. (1997). Migrant laborers and AIDS in the United States: Review of the literature. AIDS Education and Prevention, 9, 83-93.

Organista, K. C., Carrillo, H., \& Ayala, G. (2004). HIV prevention with Mexican migrants: Review, critique, and recommendations. Journal of Acquired Immune Deficiency Syndromes, 37, S227-39.

Organista, K. C., Organista, P. B., Bola, J. R., Garcia de Alba J. E., \& Moran, M. A. C. (2000). Predictors of condom use in Mexican migrant laborers. American Journal of Community Psychology, 28,245-265.

Oyserman, D., Yoder, N., \& Fryberg, S. A. (2007). Identity-based motivation and health. Journal of Personality and Social Psychology, 93(6), 1011-1027.

Painter, T. M. (2008). Connecting the dots: when the risks of HIV/STD infection appear high but the burden of infection is not known--the case of male Latino migrants in the southern United States. AIDS and Behavior, 12(2), 213-26. doi:10.1007/s10461-0079220-0

Parker, R. G., Easton, D., \& Klein, C. H. (2000, June). Structural barriers and facilitators in HIV prevention: A review of international research. AIDS (London, England).

Retrieved from http://www.ncbi.nlm.nih.gov/pubmed/10981471

Passel, J. S., Capps, R. \& Fix, M. (2004). Undocumented Immigrants: Facts and Figures. Washington, DC: The Urban Institute.

Persky, I., \& Birman, D. (2005). Ethnic identity in acculturation research: A study of multiple identities of Jewish refugees from the former Soviet Union. Journal of CrossCultural Psychology, 36, 557-572. doi: 10.1177/0022022105278542

Phinney, J. (1992). The multigroup ethnic identification measure: A new scale for use with diverse groups. Journal of Adolescent Research, 7, 156-176.

Phinney, J. S. (2003). Ethnic identity and acculturation. In K. M. Chun, P. B. Organista, \& G. Marin (Eds.), Acculturation: Advances in Theory, Measurement, and Applied Research (63-81). Washington, DC: American Psychological Association. 
Phinney, J. S. (2008). Ethnic Idenity Exploration in Emerging Adulthood. In J.J. Arnett \& J.L. Tanner (Eds.), Emerging adults in America: Coming of age in the 21 st century (117-134). American Psychological Association.

Phinney, J. S., Cantu, C. L., \& Kurtz, D. A. (1997). Ethnic and American identity as predictors of self-esteem among African American, Latino, and White adolescents. Journal of Youth and Adolescence, 26(2), 165-185. doi: 10.1023/A:1024500514834

Phinney, J. S., DuPont, S., Espinosa, C., Revill, J., Sanders, K. B. A. (1994). Journeys into cross-cultural psychology. In Bouvy, A., van de Vijver, F. J. R., Pawel, B., Schmitz, P. G. (Eds.), Journeys into cross-cultural psychology (pp. 167-183). Netherlands: Swets \& Zeitlinger Publishers.

Phinney, J. S., Horenczyk, G., Liebkind, K., \& Vedder, P. (2001). Ethnic identity, immigration, and well-being: An interactional perspective. Journal of Social Issues, 57, 493-510.

Phinney, J. S., Jacoby, B., \& Silva, C. (2007). Positive intergroup attitudes: The role of ethnic identity. International Journal of Behavioral Development, 31(5), 478-490. doi:10.1177/0165025407081466

Pitts, Byron. (2010). "Harvest of Shame” 50 years. CBS News. U.S.

Rangel, M. G., Martínez-Donate, A. P., Hovell, M. F., Santibáñez, J., Sipan, C. L., \& Izazola-Licea, J. A. (2006). Prevalence of risk factors for HIV infection among Mexican migrants and immigrants: probability survey in the North border of Mexico. Salud pública de México, 48(1), 3-12. Retrieved from http://www.ncbi.nlm.nih.gov/pubmed/16555529

Reblin, M. \& Uchino, B. N. (2008). Social and Emotional Support and its Implication for Health. Current Opinion in Psychiatry, 21(2), 201-205.

doi:10.1097/YCO.0b013e3282f3ad89

Rhodes, S. D., Bischoff, Ã. W. E., Burnell, J. M., Whalley, L. E., Walkup, M. P., Vallejos ... Arcury, T. A. (2010). HIV and sexually transmitted disease risk among male Hispanic / Latino migrant farmworkers in the Southeast: Findings from a pilot CBPR study. American Journal of Industrial Medicine. doi:10.1002/ajim.20807

Salgado de Snyder, V. N., Díaz Pérez, M., \& Maldonado, M. (1996). AIDS: Risk behaviors among rural Mexican women married to migrant workers in the United States. AIDS Education and Prevention, 8(2), 134-142.

Salomon, G. (1984). Television is "easy" and print is "tough": The differential investment of mental effort in learning as a function of perceptions and attributions. Journal of Educational Psychology, 76(4), 647-658. 
Sanchez, J. I., Fernandez, D. M. (1993). Acculturative stress among Hispanics: A bidirectional model of ethnic identification. Journal of Applied Social Psychology, 23(7):654-668.

Sánchez, M., Rice, E., Stein, J., Milburn, N. G., \& Rotheram-Borus, M. J. (2010). Acculturation, coping styles, and health risk behaviors among HIV positive Latinas. AIDS and Behavior, 14(2), 401- 409.

Schwartz, S. J., Zamboanga, B. L., \& Jarvis, L. H. (2007). Ethnic identity and acculturation in Hispanic early adolescents: mediated relationships to academic grades, prosocial behaviors, and externalizing symptoms. Cultural Diversity and Ethnic Minority Psychology, 13(4), 364-373. doi:10.1037/1099-9809.13.4.364

Seth, P., Wingood, G. M., DiClemente, R. J., \& Robinson, L. S. (2011). Alcohol use as a marker for risky sexual behaviors and biologically confirmed sexually transmitted infections among young adult African-American Women. Women's Health Issues, 21(2), $130-135$.

Shedlin, M. G., Decena, C. U., \& Oliver-Velez, D. (2005). Initial acculturation and HIV risk among new Hispanic immigrants. Journal of the National Medical Association, 97(7 Suppl), 32S-37S. Retrieved from http://www.pubmedcentral.nih.gov/articlerender.fcgi?artid=2640649\&tool=pmcentrez\&r endertype $=$ abstract

Shehadeh, N., McCoy, H. V., Rubens, M. B., Batra, A., Renfrew, R., \& Winter, K. (2011). The impact of ethnic identity on changes in high risk HIV behaviors in sexually active migrant workers. Journal of Immigrant and Minority Health, doi:10.1007/s10903011-9466-5

Taylor, D. M., \& Modhaddam, F. M. (1994). Social identity theory. In D. Taylor (Ed.), Theories of intergroup relations (2nd ed., pp. 61-94). Westport, CT: Praeger.

Temkin-Greener, H., Bajorska, A., Peterson, D. R., Kunitz, S. J., Gross, D., Williams, T. F., \& Mukamel, D. B. (2004). Social support and risk-adjusted mortality in a frail older population. Medical Care, 42(8), 779-788. doi:10.1097/01.mlr.0000132397.49094.b3

Torres, V., Martinez, S., Wallace, L. D., Medrano, C. I., Robledo, A. L., \& Hernandez, E. (2010). The connection between Latino ethnic identity and adult experiences. Adult Education Quarterly. doi:10.1177/0741713610392765

Townsend, T. G., \& Belgrave, F. Z. (2000). The impact of personal identity and racial identity on drug attitudes and use among African American Children. Journal of Black Psychology, 26(4), 421-436. doi:10.1177/0095798400026004005

U.S. Department of Health \& Human Services. (2009). HIV/AIDS Data/Statistics. 
U.S. Department of Health and Human Services. (2008). HIV/AIDS Data/Statistics. Retrieved on May 29, 2009, from

http://www.omhrc.gov/templates/browse.aspx?lvl=3\&lvlid=70

Uchino, B. N. (2006). Social support and health: A review of physiological processes potentially underlying links to disease outcomes. Journal of Behavioral Medicine, 29(4), 377-387. doi:10.1007/s10865-006-9056-5

Umaña-Taylor, A. J., \& Updegraff, K. A. (2007). Latino adolescents' mental health: exploring the interrelations among discrimination, ethnic identity, cultural orientation, self-esteem, and depressive symptoms. Journal of Adolescence, 30(4), 549-67.

doi:10.1016/j.adolescence.2006.08.002

United States. Department of Labor, Office of the Assistant Secretary for Policy. 2005. Findings from the national agricultural workers survey (NAWS) 2001-2002: A Demographic and Employment

University of California (UC). (2009). Hispanic Americans: Migrant Workers and Braceros (1930s-1964). Retrieved on May 30, 2009, from http://www.calisphere.universityofcalifornia.edu/calcultures/ethnic_groups/subtopic3b.ht $\mathrm{ml}$

Urizar, C. G., \& Winkleby, M. A. (2003). AIDS knowledge among Latinos: Findings from a community and agricultural labor camp survey. Hispanic Journal of Behavioral Sciences, 25, 295-311. doi:10.1177/0739986303256911

Viadro, C. I., \& Earp, J. A. (2000). The sexual behavior of married Mexican immigrant men in North Carolina. Social Science \& Medicine, 50(5), 723-35. Retrieved from http://www.ncbi.nlm.nih.gov/pubmed/10658852

Villarejo, D., McCurdy, S. A., Bade, B., Samuels, S., Lighthall, D., \& William, III, D. (2010). The Health of California's Immigrant Hired Farmworkers. American Journal of Industrial Medicine, 55, 387-397. doi:10.1002/ajim.20796

Villarejo, D. (2003). The health of U.S. hired farm workers. Annual Review of Public Health, 24, 175-93. doi:10.1146/annurev.publhealth.24.100901.140901

Weatherby, N. L., McCoy, H. V., Metsch, L. R., Bletzer, K. V., McCoy, C. B., \& De La Rosa, M. R. (1999). Crack cocaine use in rural migrant populations: living arrangements and social support. Substance Use \& Misuse, 34(4-5), 685-706. Retrieved from http://www.ncbi.nlm.nih.gov/pubmed/10210100

Winkleby, M. A., Snider, J., Davis, B., Jennings, M. G., Ahn, D. K. (2003). Cancer related health behaviors and screening practices among Latinos: Findings from a community and agricultural labor camp survey. Ethnicity and Disease, 13(3), 376-386. 
Worby, P. A, \& Organista, K. C. (2007). Alcohol use and problem drinking among male Mexican and Central American immigrant laborers: A review of the literature. Hispanic Journal of Behavioral Sciences, 29(4), 413-455. doi:10.1177/0739986307308765

Yoo, H. C., \& Lee, R. M. (2005). Ethnic identity and approach-type coping as moderators of the racial discrimination/well-being relation in Asian Americans. Journal of Counseling Psychology, 52(4), 497-506. doi:10.1037/0022-0167.52.4.497

Zamboanga, B. L. (2009). Acculturation and substance use among Hispanic early adolescents: investigating the mediating roles of acculturative stress and self-esteem. The Journal of Primary Prevention, 30, 315-333.

Zamboanga, B. L. (2005). Alcohol expectancies and drinking behaviors in Mexican American college students. Addictive Behaviors, 30(4), 673-84.

doi:10.1016/j.addbeh.2004.08.013

Zamboanga, B. L., Raffaelli, M., \& Horton, N. J. (2006). Acculturation status and heavy alcohol use among Mexican American college students: investigating the moderating role of gender. Addictive Behaviors, 31(12), 2188-98. doi:10.1016/j.addbeh.2006.02.018

Zambrana, R. E., Scrimshaw, S. C. M., Collins, N., \& Dunkel-schetter, C. (1997). Prenatal health behaviors and psychosocial risk factors in pregnant women of Mexican origin: The Role of acculturation. Public Health, 87(6), 1022-1027. 


\section{Chapter III \\ METHODOLOGY}

\section{Data Source}

The data used for this study were collected from a larger study, conducted from 2005 to 2010. This larger study was headed by Dr. H. V. McCoy and was focused on assessing the effectiveness of an enhanced/adapted cognitive-behavioral intervention (PEER: Peer Education Ends Risky Behavior) program compared to a health promotion comparison program (HEALTHY: Health Education Always Leads To a Healthy You) in a sample of migrant workers (MWs) and seasonal workers recruited from Immokalee, Florida. The aim of the parent study was to assess the effectiveness of these two community-based interventions in reducing HIV risk behaviors and increasing health behaviors among alcohol and other drug using migrant workers (McCoy, Hlaing, ErgonRowe, Samuels, \& Malow, 2009).

The study sample included migrant and seasonal workers residing within a 10 mile radius of Immokalee, Collier County, Florida. Inclusion criteria for both the larger study and this study was migrant or seasonal workers, age 18 or older, who reported at least one episode of unprotected (without a condom) vaginal, anal, or oral sex and had consumed drugs in the past 90 days. Participants were required to be fluent in English and/or Spanish. Seasonal migrant workers were those participants who were located in Immokalee seasonally. After recruitment, informed consent, screening and administration of baseline measures, participants were randomly assigned to one of the two interventions, PEER or HEALTHY groups. Both interventions included a four-hour workshop of the PEER or HEALTHY intervention, a post-intervention assessment, and 
follow-up assessments. Follow-up assessments were administered at the 3, 6, and 12 month follow-up periods. This study examined data from only two of the four assessment periods (baseline and 6 months) due to the ongoing project. Both interventions utilized terminology and expressions that were culturally adapted to the context of local African American and Hispanic communities in the area. The distinct focus of the PEER intervention was the peer education component and motivational counseling.

\section{Theoretical foundation}

The identity-based motivation theory best describes the link between ethnic identity and unhealthy/risky behaviors. The basis of the identity-based motivation theory is that many ethnic/racial groups perceive accepted and unaccepted behaviors on the basis of the perspective of a member of their ethnic/racial group. This theory addresses the concept of in-group behaviors. These are the practices and behaviors that many ethnic/racial groups engage in to identify themselves as belonging to their ethnic group, also known as identity-infused behaviors. These behaviors can positively or negatively influence health in a variety of ways.

Some behaviors identified as identity-infused behaviors can be demonstrated in the form of unhealthy eating habits. Unhealthy foods (fried foods, high-in-fat, and sugar foods) may be representative of a culture. The challenge in this situation is changing such unhealthy eating habits because such changes may be unaccepted by the ethnic/racial group. These behaviors are deeply embedded in a number of cultures and in many cases encourage unhealthy and high risk behaviors (Oyserman, Yoder, \& Fryberg, 2007). An example of unhealthy/risky behaviors includes the connection between the Hispanic cultural belief of "machismo" and risky sexual behaviors. Machismo reflects male pride 
which seems to push Hispanic males to want to prove their masculinity. One of the modes taken to demonstrate masculinity is sexual risk taking (Lo, Reisen, Poppen, Bianchi, \& Zea, 2011). A systematic review by Herbst et al. (2007) found that interventions that directly addressed this risky in-group concept within their intervention had greater efficacy in risk reduction in sexual behaviors than the interventions that did not. It is important to explore how such identity-infused behaviors may influence HIV risk behaviors in an understudied population like migrant workers.

The aforementioned theory is illustrated in Figure 1 below. The theory begins with the external factors of ethnic identity and demographic characteristics. The theory demonstrates that a direct relationship exists between external factors and the outcome behaviors. The outcome behaviors are divided into two types. The first type is identityinfused behaviors, which are behaviors encouraged within the ethnic culture of the individual. For the purpose of the studies presented this concept will also represent protective behaviors such as condom use. The second is HIV risk behaviors, which represents behaviors that increase the chances of HIV transmission. The key to altering risky in-group behaviors is to discover connections between ethnic/racial minority cultures and mainstream culture. The moderators specified in the diagram include the concept of social support which may strengthen the already existing relationship between the external factors and the outcome behaviors. The theory dictates that the cultural internalized norms that are connected to such high risk behaviors need to be altered. The use of the moderators may increase the possibility of achieving that change. 
Figure 1: Identity Based Motivation Theory framework

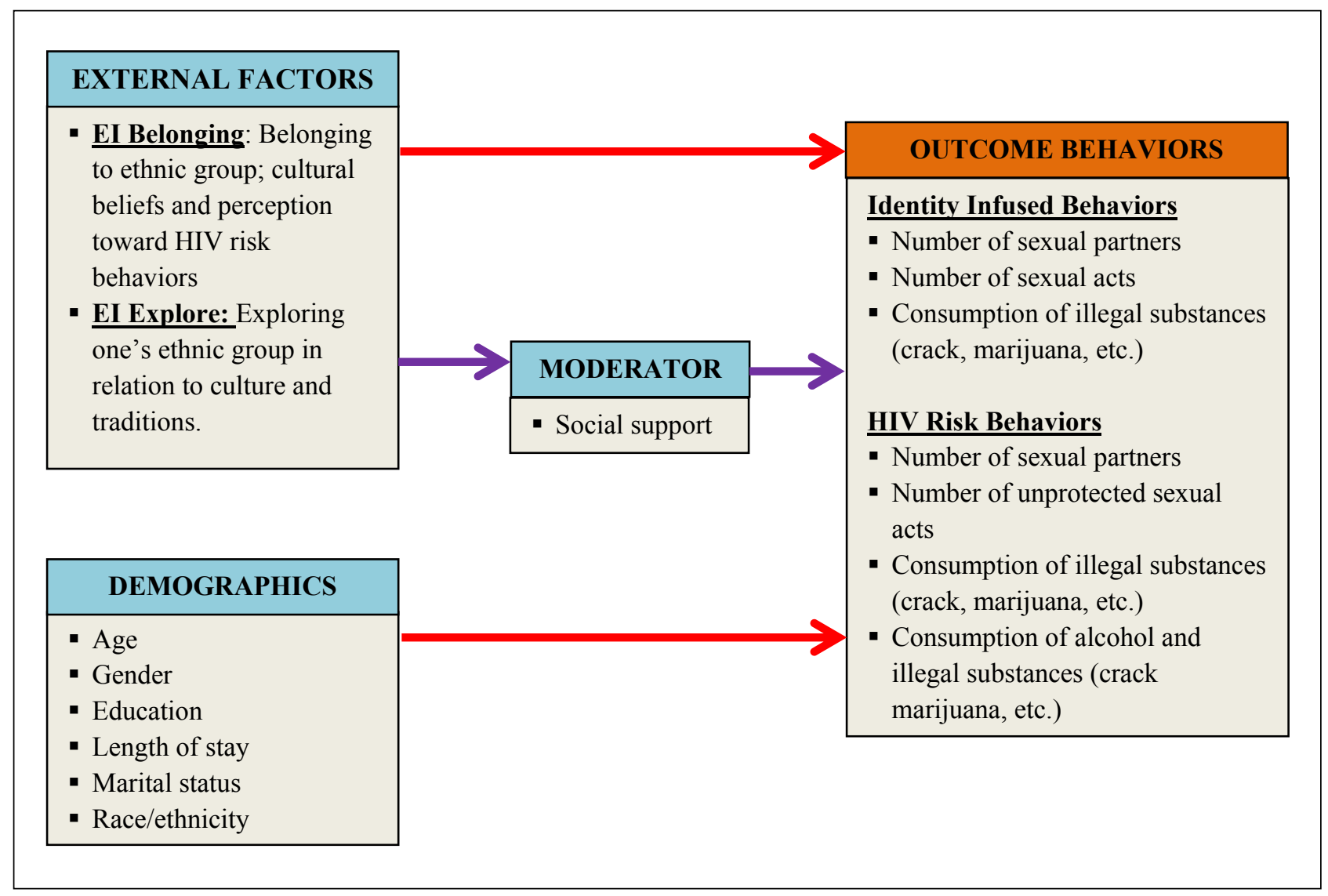

\section{Participants}

The sample was made up of 431 migrant workers and seasonal workers recruited from Immokalee, Florida, at baseline. The sample of 431, which were participants at baseline, was used in Manuscripts 1 and 2. Only 270 participants attended baseline and 6month follow-up period, the sample used for all three manuscripts. Participants resided within a 10 mile radius of Immokalee in Collier County, Florida. Criteria for both the larger study and this study included being a migrant or seasonal worker, age 18 or older, and reporting at least one episode of unprotected (without a condom) vaginal, anal, or oral sex in the past 90 days. Participants had to be fluent in English and/or Spanish. 
Participants were asked to sign an informed consent form which described the study for all participants in either English or Spanish. Participants were excluded if they were trying to get pregnant or intending to impregnate anyone. They also needed to be in the area for the following 6 months and return 6 months later.

The participants included in this study were recruited primarily from "camps," which in the context of this community may include trailer parks, dormitory-style housing, apartment buildings, motels, duplexes, and neighborhoods of single/duplex housing in the immediate and surrounding areas of Immokalee, Florida. In the setting described above, potential participants were approached and asked a few questions to see if they complied with the eligibility criteria of the HIV prevention intervention project.

\section{MEASURES}

\section{HIV/AIDS Risk Reduction Questionnaire (HRRQ)}

The HIV Risk Reduction Questionnaire is composed of 13 sections used to assess HIV risk reduction behavior and Sex Related Alcohol Expectancies (SRAE; Leigh, 1990). The sections utilized in this study are: demographics, Multigroup Ethnic Identity Measure (MEIM), sex behavior, SRAE, and alcohol and drug use.

\section{Dependent Variables}

Information was retrieved from the HRRQ utilized in the parent study. There were a total of 11 dependent variables that were analyzed in the 3 manuscripts. These dependent variables are as follows: Vaginal Episode Equivalent (VEE), number of partners, number of sexual partners without a condom, vaginal acts without a condom, alcohol consumption, marijuana usage, crack usage, SRAE total, SRAE- Enhanced Sex, SRAE-Decreased Nervousness, SRAE-Increased Risk. Independent variables that were 
continuous over the 6-month time period were: Ethnic Identity total, ethnic identity belonging, ethnic identity explore, and Social Provisions Scale (SPS) which addressed level of social support. Change variables explored in all three manuscripts were created to examine the impact of any changes, over the 6-month time period, in behaviors or beliefs that were associated with changes in HIV risk. The change variables were created by computing a new variable that calculated the difference between 6 months and baseline for the frequency of the behavior or score of the scale reported.

\section{Sexual Risk: Vaginal Episode Equivalent (VEE)}

Using data from the HRRQ, an index of sexual risk behavior, the Vaginal Episode Equivalent (VEE), was created. The VEE is an overall index representing the weighted sum of a participant's sexual acts without condoms during the past 30 days, including unprotected (without a condom) oral, anal, and vaginal acts (Susser, Desvarieux, \& Wittkowski, 1998). Compared to using individual specific sex acts (e.g., unprotected vaginal intercourse) as individual variables, this index has the advantage of providing a score reflecting a subject's overall sexual risk-taking behavior. Each sex act is weighted based on its differential risk using the following formula: VEE $=(\#$ of unprotected vaginal sex acts $)+(2 \mathrm{x} \#$ of unprotected anal sex acts $)+(0.01 \mathrm{x} \#$ of unprotected oral sex acts) (Susser et al., 1998).

The VEE is superior to an un-weighted summing of sex acts because it takes into account the fact that some behaviors are riskier than others (Susser, Desvarieux, \& Wittkowski, 1998). The VEE was computed using the recommended weightings derived from previous research (Susser et al., 1998). The higher the VEE score, the higher the sexual risk (Susser et al., 1998). For this study VEE was computed for baseline and 6- 
month follow-up. In addition, a change variable was calculated to assess the change of the VEE index over the 6-month time period by computing the difference of the scores from baseline to 6-month follow-up.

Sex-Related Alcohol Expectancies (SRAE)

Sex-Related Alcohol Expectancies (SRAE) - beliefs about alcohol's effects on sexual behavior and pleasure - were also examined (Leigh, 1990). The SRAE questionnaire is found in Appendix B. The SRAE is a 13 item questionnaire with three subscales: Enhanced Sex, Decreased Nervousness and Increased Riskiness (Appendix B). These subscales measure beliefs about whether alcohol enhances sexual pleasure (Cronbach's $\alpha=0.90$ ), decreases nervousness (Cronbach's $\alpha=0.83$ ), or increases sexual risk-taking (Cronbach's $\alpha=0.85$ ). The SRAE responses use a Likert scale ranging from 0 to 4 (not at all to very much). The Cronbach's $\alpha$ scores were derived from the current sample with an $n=270$. Subscale scores are derived by averaging the items within each of the subscales.

The first subscale, Enhanced Sex, consists of 5 items and assesses the participant's beliefs of how alcohol affects their sexual pleasure. The second subscale, Decreased Nervousness, is composed of 6 items and assesses the extent to which participants believe that alcohol helps to reduce nervousness in sexual situations. This subscale has questions such as, "I am less nervous about sex." The last subscale, Increased Riskiness, is composed of 2 items that assess an individual's belief regarding alcohol's effect on increasing risky behaviors and losing inhibitions. 


\section{Drug and Alcohol Measures}

The drug and alcohol variables included in this analysis were based on consumption during the last 30 days. These data were collected through the HRRQ. Alcohol use was measured by the number of drinks consumed in the last 30 days. Crack and marijuana use, measured by the number of times each drug was used over the previous 30 days, was also included in these analyses. The change variables computed for these substances in Manuscripts 1, 2, and 3 were created by computing the difference between consumption at baseline and 6-month follow-up.

\section{Independent Variables}

\section{Demographic Characteristic}

Demographic characteristics were collected from the HRRQ. Demographic data included in the model were age, gender, education, marital status, ethnicity, country of birth, and length of stay in the Immokalee area. Demographic data were included in the model as independent variables. Those demographic variables approaching significant $(p$ $<$.10) Pearson $r$ coefficients were included in the linear regressions conducted in this study. There was no evidence of mutlicollinearity among the predictor variables or violations of other assumptions of regression.

\section{Intervention}

An intervention variable was included in the analyses presented in this dissertation. The intervention variable was included for two reasons. First, it was included to control for the actual effects of the intervention on the outcome variables assessed and second, to assess the impact on ethnic identity by assignment of the intervention group. Although the parent study's purpose was to assess the impact of the 
intervention on sex risk behavior, it was not within the scope of the studies presented here; therefore, it was necessary to include intervention assignment as a control variable. The intervention variable is coded 1 and 2: 1 for the PEER group and 2 for the HEALTHY intervention group. This variable was used to assess the differential impact of the intervention on the full MEIM measure and the subscales, ethnic identity belonging and ethnic identity explore.

Ethnic Identity Measure: Multigroup Ethnic Identity Measure (MEIM)

The primary measurement tool utilized to assess the level of ethnic identity is the Multigroup Ethnic Identity Measure (MEIM; Phinney, 1992). The MEIM questionnaire is found in Appendix A. This scale is composed of 12 items that explore the participant's level of ethnic identity. The MEIM focuses on areas of acculturation, ethnic identification, and commitment or level of belonging to one's ethnic group. These items represent three aspects of ethnic identity: affirmation/belonging; ethnic identity achievement; and ethnic behaviors and practices. Item responses use a Likert scale, ranging from 1 (strongly disagree) to 4 (strongly agree). The MEIM score gives an indication of the comfort level participants have with the individuals, beliefs, and practices from their ethnic group (Phinney, 1992). A mean score was calculated to assess the level of ethnic identity of each participant; the higher the mean MEIM score, the more comfortable participants felt with their ethnic group. Cronbach's alpha of the scale was 0.85 . The scale has been utilized in numerous articles that are cited in this dissertation (Espinosa-Hernandez \& Lefkowitz, 2010; Phinney, 1992; Utsey, Chae, Brown, \& Kelly, 2002;). 
Continuous composite variables were created for the total MEIM measure, and the subscales. The continuous change variables for ethnic identity were computed by using the mean ethnic identity score of the participant at two time points, baseline and 6month follow-up.

The MEIM has two subscales that are included throughout the analyses: ethnic identity belonging and ethnic identity explore. Ethnic identity belonging examines the extent to which individuals feel a positive sense of belonging with their ethnic group. This subscale is composed of 7 items from the MEIM measure.

The ethnic identity explore component measures the degree to which individuals explore or take an active role to become better acquainted with and learn about their ethnic group. Ethnic identity explore was created by taking the mean of 5 items from the MEIM scale.

\section{Social Support: Social Provisions Scale (SPS)}

Social support was assessed by using the Social Provisions Scale (SPS) (Cutrona \& Russell, 1987). The SPS examines the degree to which participants' social relationships provide various dimensions of social support. This 24 -item scale is a multidimensional construct; the SPS questionnaire is found in Appendix C. The responses range from 1 (strongly agree) to 5 (strongly disagree). This measure can be divided in two different ways. Assisted-related SPS falls under the category of social relationships directly related to problem solving, such as guidance. This represents the feeling that people can be counted on when the individual is under stress. The second category, nonassisted related SPS, is beneficial to the individual under both low and high stress situations, but does not include problem solving assistance. Change variables for 
Manuscript 2 were computed by taking the SPS total and computing the difference between 6-month and baseline scores.

\section{Statistical Analysis}

The data analyzed for the three manuscripts presented in this dissertation involved the use of bivariate correlations, repeated measures ANCOVA (RMANCOVA), and multiple linear regressions. Those demographic variables that were found to have close to significance $(p<.10)$ Pearson $r$ coefficients were included in the linear regressions conducted in this study. Table 1.2 and Table 2.2, found in Manuscript 1 and Manuscript 2, display the Pearson $r$ coefficients for each outcome variable. There was no evidence of mutlicollinearity among the predictor variables.

Research Question $1 \& 2$

Pearson correlations and multiple linear regressions were executed to assess associations between ethnic identity and risky sexual behaviors, alcohol use, drug use, and alcohol expectancy measures. Demographics such as ethnicity, marital status, gender, education, migration status, as well as the intervention variable were included in the models as covariates.

Multiple linear regression analyses were conducted on a number of hypotheses in this dissertation. A $\log 10$ transformation was performed on the variables with high kurtosis levels in order to retain maximum power and to ensure that none of the assumptions for linear regression models were violated.

Multiple linear regressions were used in the studies presented in this dissertation in order retain the highest level of power in the statistical analyses conducted. The variables in the data set were assessed to determine if they met the assumptions for linear 
regression analysis. Violations to these assumptions were minimal and corrected by log 10 transformations. It is important to be aware of how "robust" the linear regression analysis is. However, if one or more assumptions are not met, it has been reported that unbiased and efficient results will still be achieved (Edgell \& Noon, 1984; Havlick \& Peterson, 1977; Hayes, 1996). An issue arises, however, when the sample size is less than 120 and there is extreme non-normality in the variables analyzed. This was not the case in the migrant worker data set used in this analysis (Edgell \& Noon, 1984; Havlick \& Peterson, 1977; Hayes, 1996). Transformations were conducted on the variables that presented non-normality characteristics in order to reduce any potential loss of power.

\section{Research Questions $3 \& 4$}

Repeated Measures Analysis of Variance (RMANCOVA) was used to control for group design (PEER v. HEALTHY) when assessing ethnic identity change baseline and 6-month follow-up as well as the interaction between ethnic identity change and intervention assignment group. SPS was included in the linear regression analyses. Demographics such as ethnicity, marital status, gender, education, migration status, and the intervention variable were included in the models as covariates. The covariates included in each model were dependent on the significance of the correlation coefficient. The correlation had to comply with an approaching significant $p$-value of .10 in order to be included in the regression analyses.

Missing

Missing data was minimal throughout the data set utilized for this study, not exceeding a range of $0-2 \%$. A mean replacement method was used to rectify this issue. 


\section{Loss to Follow-Up}

Manuscript 1 and Manuscript 2 had participants that were lost. One hundred sixty-one participants from the original data set were not included in the analyses since they did not have 6-month follow-up data; about $37 \%$ of the participants at baseline were lost. These lost to follow-up individuals included 32 African Americans (67\% male) and 129 Hispanics (84\% male). Approximately $81 \%$ of the African Americans were single compared to $43 \%$ of the Hispanic participants. The mean education level for these participants was 7.37 grade $(S D=3.57)$ and the average age was 39.86 years $(S D=$ 11.48). The mean number of risky sexual behaviors among this group was as follows: 3.84 vaginal acts in the last 30 days $(S D=7.74)$ and 4.06 sexual partners in the last 30 days $(S D=17.43)$. These participants had a mean of 101.09 drinks in the last 30 days $(S D=140.44)$. With regard to substance use, $60 \%(n=96, M=10.72$ times in the last 30 days, $S D=31.98)$ consumed marijuana in the last 30 days, while $34 \%(n=55, M=27.55$ times in the last 30 days, $S D=85.91$ ) consumed crack in the last 30 days.

There were 270 study participants with both baseline and 6-month data included in these analyses. The sample was composed of both Hispanic $(n=142,52 \%)$ and African American $(n=128,48 \%)$ ethnicities. The sample is composed of $67 \%(n=180)$ males and 33\% $(n=90)$ females. The average achieved educational level was 9.19 grade $(S D=3.10)$. The means computed for baseline risky behaviors were as follows: 3.36 vaginal acts without a condom in the last 30 days $(S D=6.97)$ and 2.14 sexual partners in the last 30 days $(S D=4.32)$. Approximately 54 alcoholic drinks were consumed in the last 30 days by the migrant workers in the sample. The participants consumed crack 
approximately four times in the last 30 days $(n=57 ; S D=23.34)$, while marijuana was consumed approximately seven times in the last 30 days $(n=109 ; S D=15.91)$.

When comparing cases not included in the analyses and those followed, there were a number of significant differences between the groups. The lost cases were significantly younger $(M=39.86)$ than the cases that participated $(M=42.58)$ in the 6month assessment period $(t=-2.172, p=.027)$, and lost cases had a significantly lower level of education $(M=7.37)$ compared to participants $(M=9.19)$ included in this study $(t=-5.207, p<.001)$. A large portion of the lost cases were of Hispanic ethnicity $(80 \%, p$ $\left.<.001, \chi^{2}=31.99\right)$, and lost cases consumed significantly larger amounts of crack in the last 30 days compared to the followed cases $(M=27.55 \mathrm{v} . M=4.01$, respectively; $t=$ $4.209, p<.001)$.

Sample Size and Power Analysis

The parent study had a sample size at baseline of 431 participants, which is the sample used for baseline data on substance use in Manuscript 1 and baseline data on risky sexual behaviors in Manuscript 2. A post-hoc power analysis was conducted with G*Power 3 software (Faul, Erdfelder, Lang, \& Buchner, 2007). A medium effect size of .15 and an $\alpha$ of .05 yielded a power of .999 . The formula used was: power $=1-\beta$. This level of power provides enough strength to assume that the number of participants was sufficient for the analysis conducted in this study.

The sample size used to analyze change at 6-month follow-up for Manuscripts 1, 2, and 3 was 270 participants which contained those who participated in baseline and 6month follow-up sessions. One hundred sixty-one participants were lost because they did not complete the 6-month follow-up assessment. The software utilized for the power 
analyses was G*Power 3 (Faul, Erdfelder, Lang, \& Buchner, 2007). The power analysis conducted included a medium effect size with an $\alpha$ of .05 and power of .95 yielding a suggested minimum sample size of 178 . This requirement was met and statistical analysis was conducted on the sample. The power analysis for each regression reported in Manuscripts 1, 2, and 3 are displayed in tables found in Appendix 3.

Software

The statistical software utilized for the analyses conducted in the studies presented was IBM SPSS Statistics 19 (IBM Corporation). 


\section{REFERENCES}

Cutrona, C. E. \& Russell, D. W. (1987). The provisions of social relationships and adaptation to stress. Advances in Personal Relationships, 1, 37-67.

Edgell, S. E., \& Noon, S. M. (1984). Effect of violation of normality on the $t$ test of the correlation coefficient. Psychological Bulletin, 95(3), 576-583. doi:10.1037//00332909.95.3.576

Faul, F., Erdfelder, E., Lang, A.-G., \& Buchner, A. (2007). G*Power 3: A flexible statistical power analysis for the social, behavioral, and biomedical sciences. Behavior Research Methods, 3(2)9, 175-191. doi: 10.3758/BF03193146

Havlick, L.L. \& Peterson, N. L. (1977). Effect of the violation of assumptions upon significance levels of the Pearson r. Psychological Bulletin, 84(2), 373-377.

Hayes, A. F. (1996). Permutation test is not distribution-free: Testing H-sub-0: $\rho=0$. Psychological Methods. doi:10.1037/1082-989X.1.2.184

Herbst, J. H., Kay, L. S., Passin, W. F., Lyles, C. M., Crepaz, N., Marín, B. V., \& the HIV/AIDS Prevention Research Synthesis (PRS) Team (2007). A systematic review and meta-analysis of behavioral interventions to reduce HIV risk behaviors of Hispanics in the United States and Puerto Rico. AIDS and Behavior, 11(1), 25-47.

doi:10.1007/s10461-006-9151-1

Leigh, B. C. (1990). The relationship of sex-related alcohol expectancies to alcohol consumption and sexual behavior. British Journal of Addiction, 85(7), 919-28.

doi: 10.1111/j.1360-0443.1990.tb03722

Lo, S. C., Reisen, C. A., Poppen, P. J., Bianchi, F. T., \& Zea, M. C. (2011). Cultural beliefs, partner characteristics, communication, and sexual risk among latino MSM. AIDS and Behavior, 15(3), 613-20. doi:10.1007/s10461-010-9760-6

McCoy, H. V., Hlaing, W. M., Ergon-Rowe, E., Samuels, D., \& Malow, R. (2009). Lessons from the fields: a migrant HIV prevention project. Public health reports, 124(6), 790-6. Retrieved from http://www.ncbi.nlm.nih.gov/pmc/articles/PMC2773941/

Otto-Salaj, L. L., Heckman, T. G., Stevenson, L. Y., Kelly, J. A., \& (1998). Patterns , predictors and gender differences in HIV risk among severally mentally ill men and women. Community Mental Health Journal, 34(2), 175-190. doi: 10.1023/A:1018745119578

Oyserman, D., Fryberg, S. A, \& Yoder, N. (2007). Identity-based motivation and health. Journal of Personality and Social Psychology, 93(6), 1011-27. doi:10.1037/00223514.93.6.1011 
Phinney, J. S. (1992). The multigroup ethnic identity measure: A new scale for use with diverse groups. Journal of Adolescent Research, 7(2), 156-176.

doi:10.1177/074355489272003

IBM Corporation. (2010). IBM SPSS Base 19.0 for Windows User's Guide. IBM Corporation, New York.

Susser, E., Desvarieux, M., \& Wittkowski, K. M. (1998). Reporting sexual risk behavior for HIV: a practical risk index and a method for improving risk indices. American Journal of Public Health, 88(4), 671-4. doi: 10.2105/AJPH.88.4.671

Utsey, S., Chae, M. H., Brown, C. F., \& Kelly, D. (2002). Effect of ethnic Group membership on ethnic identity, race-related stress, and quality of life. Cultural Diversity and Ethnic Minority Psychology, 8(4), 366-377. doi:10.1037/1099-9809.8.4.367

Weiss, R. S. (1974). The Provisions of Social Relationships. In Z. Rubin (Ed.), Doing Unto Others (pp. 17-26). Englewood Cliffs, NJ: Prentice-Hall. 


\section{Chapter IV}

\section{TRANSITION TO MANUSCRIPT 1}

This dissertation is written in manuscript style with three publishable articles. It was inevitable that some information would be excluded from the publishable article portion of each manuscript since publishers require short articles. Manuscript 1 includes all the significant multiple linear regressions. This transition section includes the results of analyses which found no significant relationship between ethnic identity and HIV risk behavior/alcohol expectancy measures. The dependent variables of the regressions which were not reported in detail in the manuscript were number of sexual partners in the last 30 days, number of unprotected sexual partners in the last 30 days, marijuana use in the last 30 days, the total measure of the Sex Related Alcohol Expectancy (SRAE) and SRAE subscales, Enhanced Sex and Decreased Nervousness. These regressions are described in Tables 1.7 through 1.12, found in Appendix 5, in detail.

The following section is Manuscript 1 of this dissertation. The information most relevant to the proposed hypotheses was reported in the manuscript. 


\title{
MANUSCRIPT 1
}

\section{THE ROLE OF ETHNIC IDENTITY ON ALCOHOL AND DRUG USE AMONG MIGRANT WORKERS: IS IT PROTECTIVE?}

\begin{abstract}
Background: Among migrant workers in the U.S., the prevalence of HIV/AIDS is estimated to be as high as $13 \%$ among drug users. Alcohol and drug use have been associated with increasing one's risk of engaging in unprotected sex, which increases the risk of HIV transmission. This longitudinal study examines the relationship between ethnic identity and alcohol use, drug use, and beliefs among a sample of African American and Hispanic migrant workers.

Methods: Baseline data was collected from a larger HIV intervention study among migrant workers in Immokalee, Florida $(n=431)$. Six month-follow-up $(n=270)$ data was also used to analyze change in behaviors and ethnic identity. Two subscales of the Multigroup Ethnic Identity Measure (MEIM), which assesses the level of connection with one's ethnic group, were utilized in these analyses. Multiple linear regressions were conducted to assess the relationship between ethnic identity and alcohol use, drug use, and alcohol expectancy measures. Alcohol abuse risk beliefs were measured by the Sex Related Alcohol Expectancy Measure (SRAE), subscales of SRAE were included in the analyses.
\end{abstract}

Results: Ethnic identity subscales, explore and belonging, significantly predicted crack use. Ethnic identity explore also significantly predicted alcohol use and alcohol expectancy. Ethnic identity did change over time. Ethnic identity change was significant, 
but with a small effect size. Increases in ethnic identity explore were found to be associated with a reduction in alcohol use and increased risk alcohol expectancy scores. Discussion: These findings suggest that ethnic identity may play a protective role in migrant workers 'consumption of alcohol and drug use; however, further research is needed to ascertain the strength of this relationship.

Key Words: Migrant Workers, ethnic identity, behavior change, HIV/AIDS prevention 


\section{INTRODUCTION}

Migrant workers are one of the most rapidly growing populations in the United States (U.S.) and have been significantly affected by HIV/AIDS (Coppel, Dumont, \& Visco, 2001; Villajero et al., 2010). More than 9 million people in the U.S., primarily concentrated in Texas, Florida, Washington, California, Oregon, and North Carolina, are migrant farm workers (Carroll et al., 2005; Coppel et al., 2001; Fitzgerald, Chakraborty, Shah, Khuder, \& Duggan, 2003; P.B. Organista, K. C. Organista, \& Soloff, 1998; Passel, Caps, \& Fix, 2004; Rhodes et al., 2010). The South Florida region has been severely affected by the HIV epidemic, with elevated risks for the infection being detected in the migrant worker community (Fernandez et al., 2009). The national seroprevalence rate of HIV infection is 0.4 percent, and the estimated HIV seroprevalence rates among migrant workers are between 2.6 to 13\% (Gwyther, 1998; Villarejo, McCurdy, Bade, Samuels, Lighthall, \& Williams, 2010).

Alcohol and illegal substance abuse have been identified as unhealthy coping mechanisms practiced among migrant workers (Hansen \& Donohoe, 2003; Weatherby et al., 1997). Apostolopoulos and colleagues (2006) suggested that a primary coping

mechanism used among Mexican migrant workers is consuming alcohol. Drugs became a secondary coping mechanism since drugs became more available to them after crossing the border into the U.S. A study by Duke and Carpinteiro (2009) found that migrant workers who have been separated from their families for a long period of time were more likely to have alcohol abuse problems, ultimately leading to riskier sexual behaviors (Duke and Carpinteiro, 2009). Fernandez and her colleagues (2004) suggested that migrant workers who consumed marijuana or cocaine were at higher sexual risk for HIV 
transmission, which was indicated as a possible coping behavior (Fernandez et al., 2004). There is a limited amount of research that focuses on migrant workers and alcohol and drug use, but as more research is being conducted, it is becoming evident that substance abuse is a critical issue among this underserved population.

The altered mental status linked to alcohol and drug use has been associated with risky sexual behaviors, such as unprotected sexual acts (Cooper, 2002; Noriis, Masters, \& Zawacki, 2004; Seth, Wingood, DiClemente, \& Robinson, 2011). Previous research has suggested that there is a link between drug abuse and risky sexual practices (Brown \& Van Hook, 2006; Duncan, Boisjdy, Kremer, Levy, Eccles, 2005). Literature on the association between substance use and ethnic identity is scarce.

\section{Ethnic Identity}

Studies have posited that ethnic identity may be associated with positive health behaviors such as healthy lifestyle practices. Such healthy lifestyle practices include low consumption of alcohol, no/low substance use, and practicing HIV preventative behaviors (Brook, Whiteman, Balka, Win, \& Gursen, 1998; Nesdale, Rooney, \& Smith, 1997). Ethnic identity, as defined by Phinney, DuPont, Espinosa, Revil, and Sanders (1994), is “a feeling of belonging to one's group, a clear understanding of the meaning of one's membership, positive attitudes toward the group, familiarity with its history and culture and involvement in its practices" (p.169). Ethnic identity takes into account aspects of ethnic involvement such as language, behavior, values, and knowledge of ethnic group history (Persky et al., 2005; Salgado de Snyder, Díaz Pérez, \& Maldonado, 2006). Phinney further defines the two components of ethnic identity as ethnic identity exploration and ethnic identity belonging (Phinney, 1992). These two components are 
from Phinney's Multigroup Ethnic Identity Measure (MEIM) and are the primary measures used in this study. Ethnic identity "belonging" contains ethnic pride, positive feelings toward one's ethnic group, and one's satisfaction with being a member of his/her ethnic group. Ethnic identity "explore" is the second component which measures the extent to which an individual searches for a sense of meaning in his/her ethnicity (Phinney, 1992).

Ethnic identity is distinguished from acculturation on the basis that ethnic identity measures the level of attachment one has with his/her ethnic group while acculturation measures how one from an ethnic/minority group adapts to the mainstream culture. Many studies use acculturation and ethnic identification as one construct, even though it is crucial to recognize their differences. Acculturation is associated with changes in attitudes or beliefs in relation to the mainstream or dominant culture. This concept is usually measured at a group level, while ethnic identification is assessed at the individual level. The majority of studies that measure acculturation fail to assess the multidimensional component of this construct (Abraido-Lanza et al., 2006). Ethnic identification measures the individual's emotional attachment (comfort level) and the engagement in cultural practices of his/her ethnic group (Phinney, 1992).

While investigations on ethnic identity are scarce, exploring changes in ethnic identity is even more uncommon. A limited number of studies have explored ethnic identity changes in adulthood. Those studies concluded that changes in ethnic identity can occur over time (Phinney, 2007; Torres et al., 2010). For example, Torres and colleagues (2010) found that ethnic identity changes in Latinos during adulthood and attribute the changes to changes in the environment. New encounters and life altering 
obstacles may bring about a developmental change in one's identity which will affect ethnic identity. This change in ethnic identity was named "identity reconstruction" by Marcia (1966, p.15). A change in ethnic identity revolves around reevaluating one's identity and attachment to one's ethnic group. Among adults, therefore, it seems that environmental instability can ultimately lead to changes in ethnic identity. Ethnic identity change has not been examined for associations with positive or negative health outcomes (Phinney, 2007; Torres et al., 2010).

\section{Ethnic Identity and Alcohol and Other Drugs}

Alcohol and drug use, and their relationship to ethnic identity have been minimally studied. The studies on ethnic identity and alcohol have shown that an inverse relationship seems to exist between ethnic identity and alcohol and/or drug use. For example, higher levels of ethnic identity were associated with lower levels of substance use (Beadnell et al., 2003; Townsend and Belgrave, 2000). Townsend and Belgrave (2000) examined racial identity in African American children. The Children's Black Identity Scale was used to measure how closely a child identifies with his/her racial group. The results suggested that higher levels of racial and personal identity were negatively associated with drug use and attitudes toward drug use. Similar to ethnic identity, higher levels of racial identity were associated with lower levels of drug use. Beadnell et al. (2003) examined the extent that baseline levels of ethnic identity offset negative psycho-behavioral characteristics in African American adolescents, ultimately affecting their substance abuse practices in a positive manner (Beadnell et al., 2003). This study also explored how ethnic identity would affect outcomes in an HIV risk reduction intervention, finding that ultimately those with higher ethnic identity at baseline had more 
positive outcomes in sexual behaviors over a four month period compared to participants with low ethnic identity (Beadnell et al., 2003).

Brook and Pahl (2005) examined ethnic identity and drug use in an adult African American sample. They concluded that the higher the attachment to the ethnic group or the higher the level of ethnic pride (i.e., same as ethnic identity), the lower the level of drug use and symptoms of depression. Similar findings were found by Brook and colleagues (1998) in Hispanic samples. Their study suggested that ethnic identity had a protective effect among Hispanic adolescents. Higher ethnic identity was associated with less risk in drug use behaviors (Brook, Whiteman, Balka, Win, \& Gursen, 1998).

Other studies report results opposite to those studies assessing alcohol use and ethnic identity discussed above. Hispanic males have been reported to have high levels of alcohol consumption if they have a high level of ethnic identity (Albarran \& Nyamathi, 2011; Worby \& Organista, 2007). The interpretation of this finding was that alcohol consumption seems to be encouraged in the Hispanic culture, especially among males. The norm may be in opposition to the protective role of ethnic identity since in this case, higher levels of ethnic identity coincide with cultural norms (Zamboanga, Rafaelli, \& Horton, 2006).

Studies have posited that ethnic identity may be associated with health promoting behaviors, which may consist of low consumption of alcohol, low/no substance use, and practicing HIV prevention behaviors (Brook, Whiteman, Balka, Win, \& Gursen, 1998; Nesdale, Rooney, \& Smith, 1997). These studies demonstrated that people living with HIV who had higher levels of ethnic identity not only had lower levels of psychological stress, but also found stronger social support systems and had higher levels of self- 
efficacy of coping strategies (Crawford, Allison, Zamboni, \& Soto, 2002; Lopez, Antoni, Fekete, \& Penedo, 2010). The expectation in the study reported in this paper is that ethnic identity may play a similar role for migrant workers since the migrant worker sample is composed of African American and Hispanic participants that consume alcohol, marijuana, and crack.

The focus of this study is to assess whether ethnic identity positively or negatively influences risky behaviors, such as alcohol and drug use, which increase the chances of unprotected sex while increasing the risk of HIV transmission. This research explores three topics which may impact HIV risk: (1) does an inverse relationship exist between ethnic identity belonging and ethnic identity explore and alcohol use, drug use (crack and marijuana), and beliefs in the effects of alcohol; (2) does ethnic identity change over a 6month time period, and if so, (3) do changes in ethnic identity explore and ethnic identity belonging influence changes in alcohol use, drug use (marijuana and crack), and the beliefs of the effects of alcohol on sexual behaviors in a positive (protective) manner? A deeper understanding of ethnic identification, through the use of ethnic identity subscales, may assist in determining specific factors that may influence substance use among migrant workers.

\section{METHODS}

Baseline $(n=431)$ and 6-month follow-up $(n=270)$ data used for this study were obtained from a larger HIV Risk Reduction Intervention project, conducted from 2005 to 2010 (McCoy, Hlaing, Ergon-Rowe, Samuels, \& Malow, 2009). Baseline data was collected before the actual intervention was implemented. 
The migrant worker definition utilized for this study conforms to the Public Health Services Act (1944) definition. The Public Health Services Act states that migrant workers are those individuals who are employed in agricultural labor, either seasonal or migratory, and live in temporary housing. The study criteria did not limit the definition of migrant workers to those who travel 75 miles or cross county lines to work. The study also included people who work or worked indirectly in agriculture, such as in packing houses.

The theory that guided this study is known as the Identity Based Motivation Theory (Oyserman, Yoder, \& Fryberg, 2007). This theory focuses on how ethnic/racial groups perceive accepted and unaccepted behaviors based on the perspective of a member of their ethnic/racial group known as identity infused behaviors. This theory best described the link that exists between ethnic identity and unhealthy/high risk behaviors. In many cases identity infused behaviors are protective in relation to unhealthy behaviors.

\section{Participants}

The baseline sample was made up of 431 migrant and seasonal workers, while the 6-month follow-up data was composed of 270 migrant and seasonal workers. The participants were recruited from Immokalee, Florida. Participants resided within a 10 mile radius of Immokalee in Collier County, Florida. Inclusion criteria for the parent study and this study included being a migrant or seasonal worker, age 18 or older, and reporting at least one episode of unprotected vaginal, anal, or oral sex in the past 90 days. Participants had to be fluent in English and/or Spanish. Participants were asked to sign an informed consent form which briefly described the study for all participants in either English or Spanish. Participants were excluded if they were trying to get pregnant or 
intending to impregnate anyone. They also needed to be in the area for the following 6 months and to return for the 6-month follow-up.

The participants included in this study were recruited from "camps." In the community found in Immokalee, "camps" referred to trailer parks, dormitory-style housing, apartment buildings, motels, duplexes and neighborhoods of single/duplex housing in the immediate and surrounding areas. In this setting, potential participants were approached and asked a few questions to see if they complied with the eligibility criteria of the HIV prevention intervention project.

\section{Measures}

\section{Dependent Variables}

The dependent variables were self-reported and collected through the HIV/AIDS Risk Reduction Questionnaire (HRRQ) which was composed of 204 questions and covered 13 areas. The sections of the questionnaire from which the dependent variables were retrieved are: alcohol and drug use and the Sex Related Alcohol Expectancy (SRAE) measure. The dependent variables included in the regression models were alcohol consumption and alcohol consumption change, marijuana use and marijuana use change, crack use and crack use change, SRAE scores and SRAE change scores and the SRAE subscales and change variables for each subscale, SRAE-enhanced sex, SRAEdecreased nervousness and SRAE-increased risk.

Alcohol consumption referred to the number of drinks in the last 30 days. Marijuana and crack use included times used in the last 30 days. The total score for the SRAE measures the participant's beliefs about alcohol's effects on sexual behavior and pleasure. The SRAE (Cronbach's $\alpha=0.91$ ) is composed of 13 items and ranges from 0 to 
4 (not at all to very much). Subsets of the SRAE measures beliefs about whether alcohol increases sexual riskiness (Cronbach's $\alpha=0.85$ ), decreases nervousness (Cronbach's $\alpha=0.83$ ), and enhances sexual pleasure (Cronbach's $\alpha=0.90$ ). Change variables were created for each dependent variable to determine the magnitude of changes over the 6month time period. The change variables were created by computing a new variable that calculated the difference between 6 months and baseline for the frequency of the behavior or score of the scales reported.

\section{Independent Variables}

The Multigroup Ethnic Identity Measure (MEIM) was used to assess participants' ethnic identity level (Phinney, 1992). The MEIM is a 12-item scale that measures a participant's level of attachment with his/her ethnic group into the surrounding society. The MEIM questionnaire analyzes three aspects of ethnic identity: belonging toward participant's ethnic group, ethnic identity achievement, and ethnic behaviors and practices. The items were divided into two subscales which were used in this study: ethnic identity explore and ethnic identity belonging. Ethnic identity belonging was composed of seven items from the MEIM scale, and examined the extent to which an individual feels a sense of positive belonging to his/her ethnic group. Ethnic identity explore measured the aspect of the MEIM scale that measures the degree to which an individual explores or takes an active role to become better acquainted with and learn about his/her ethnic group. Ethnic identity explore consisted of five items from the MEIM scale. The MEIM score also indicated a participant's comfort level with individuals from his or her own ethnic group or from the mainstream culture of the residing area (Phinney, 1992). Dichotomous variables were created for ethnic identity 
belonging and ethnic identity explore at baseline and for change by splitting the continuous variable into two groups: low and high or no/negative change and positive change. The four dichotomous variables created were used to analyze any differences with demographic characteristics of the sample.

The ethnic identity measure used followed a Likert scale, ranging from 1 (strongly disagree) to 4 (strongly agree). A mean score was calculated to measure the level of ethnic identity of each participant for each of the subscales; the higher the mean MEIM score, the higher the participant's ethnic identity. Cronbach's alpha for the MEIM measure was 0.85 .

The demographic variables included in the models were age, gender, education, country of birth, length of stay (reflects length of stay in Immokalee), and ethnicity. Demographic variables were included in the regression analysis as independent variables only if a correlation approaching significance $(p<.10)$ with the selected substance use variables and alcohol expectancy measures was found. Pearson $r$ coefficients for each outcome variable with the demographic variables are displayed in Appendix 1.

\section{Statistical Analysis}

The data was analyzed using bivariate correlations, multiple linear regressions, and Repeated Measures ANCOVA. Multiple linear regression were executed to illustrate the nature of the relationship, if one exists, between ethnic identity belonging and ethnic identity explore with alcohol and drug use. Multiple linear regressions were used in the studies presented in this dissertation in order retain the highest level of power in the statistical analyses conducted. The variables in the data set were assessed to determine if they met the assumptions for linear regression analysis. Alcohol use, crack use, and 
marijuana use were the outcome variables that did not meet the assumptions of linear regression due to high kurtosis levels. Log 10 transformations were executed for those variables because they did not meet the assumptions of linear regression.

The outcome variables of the multiple linear regressions were alcohol consumption, marijuana use, crack use, SRAE, SRAE-enhanced sex, SRAE-decreased nervousness, SRAE-increased risk with ethnic identity belonging and ethnic identity explore at baseline. The first block of each linear regression was composed of the demographic variables (age, gender, language, education, length of stay in Immokalee, marital status, and/or ethnicity) that were correlated with the outcome variable (alcohol consumption, marijuana use, crack use, SRAE, SRAE-enhanced sex, SRAE-decreased nervousness, SRAE-increased risk). Pearson $r$ coefficients for the bivariate correlations conducted are displayed in Appendix 1. The second block of the regression was composed of ethnic identity belonging and ethnic identity explore. Means and standard deviations for each independent variable for both data sets are found in Table 1.1 above. Similar regressions were conducted analyzing the relationship between changes in the previously mentioned outcome variables with changes in ethnic identity explore and ethnic identity belonging at the 6-month follow-up period. Ethnic identity belonging change, ethnic identity explore change, and demographic data were included as independent variables in the linear regressions. The intervention variable (whether the participant was in the PEER or HEALTHY intervention) was controlled for by including it in all linear regressions executed. The first block of each linear regression was composed of the demographic variables (i.e., age, gender, language, education, length of stay in Immokalee, marital status, and/or ethnicity and intervention assignment) that were 
correlated with the alcohol, marijuana, and crack use as well as alcohol expectancy measures (displayed in Appendix 1) and the second block was composed of ethnic identity belonging change and ethnic identity explore change. Change variables were computed for the dependent variables (alcohol consumption, marijuana use, crack use, SRAE, SRAE-enhanced sex, SRAE-decreased nervousness, SRAE-increased risk) and for ethnic identity belonging and ethnic identity explore, as previously mentioned. Missing

Missing data was very minimal throughout the data set utilized for this study, not exceeding a range of $0-2 \%$. A mean replacement method was used to replace missing data.

\section{Sample Size and Power Analysis}

This study utilized the same sample participants at two time points, baseline ( $n=431)$ and 6-month follow-up $(n=270)$. A post-hoc power analysis was conducted with G*Power 3 software (Faul, Erdfelder, Lang, \& Buchner, 2007) for both data sets used yielding a power between .955 and .999. This level of power provides enough strength to assume that the number of participants was sufficient for the analysis conducted in this study. The power analysis for each outcome variable analyzed as reported in this manuscript is displayed in Appendix 3.

\section{RESULTS}

\section{Demographics}

The baseline migrant worker sample was similar to the ethnic composition of migrant workers in the U.S. with 75\%, the largest composition, being of Mexican ethnicity and 25\% being from other ethnicities within the U.S. (National Agriculture 
Worker Survey, 2010). The breakdown by ethnicity for the migrant worker sample used in this study was 37.4\% African American and 62.6\% Hispanic (primarily Mexican). The mean age of the full sample $(n=431)$ was 41.56 years $(S D=12.39)$. Hispanics $(M=$ 38.79, $S D=11.65)$ were significantly younger than African Americans $(M=46.22, S D=$ $12.24 ; t=6.29, p<.001)$ in the sample. The overall educational level was generally low in both ethnic groups. The mean level of educational achievement for the full sample was $8.45(S D=3.39)$. However, Hispanics $(M=7.30 ; S D=3.25)$ were found to have a significantly lower educational level that African Americans $(M=10.39, S D=2.66 ; t=$ $10.20, p<.001)$.

Overall, the participants that were lost to follow-up engaged in riskier behaviors than participants included in the 6-month follow-up. Participants lost to follow-up were different from participants who were followed at 6 months on a number of levels. Lost participants were significantly younger $(M=39.86, S D=11.48 ; t=-2.97, p=.023)$ and less educated $(M=7.37, S D=3.57 ; t=-5.108, p<.001)$. Those lost to follow-up engaged in much riskier levels of crack use $(M=31.02, S D=92.40 ; t=4.806, p<.001)$ than those participants who were followed. Those participants lost to follow-up consumed about the same amount of alcoholic drinks $(M=101.09, S D=140.44)$ as the baseline data set $(M=103.77, S D=138.32)$ and 6-month data set $(M=54.42, S D=$ 71.83). Table 1.1 displays the mean and standard deviations for the demographic variables for baseline, 6-month follow-up, and those participants lost to follow-up samples. The means and standard deviations for the demographic variables and alcohol use and drug use (marijuana and crack) by ethnicity at baseline and at 6-month follow-up 
are displayed in Appendix 4. No significant difference was found between ethnicities in risky sexual behaviors. 
Table $1.1(1)$

Baseline, 6 month follow-up, and Loss-to-Follow Up data sets by Demographics

\begin{tabular}{|c|c|c|c|c|}
\hline Measure & Baseline $(n=431)$ & $\begin{array}{l}6 \text { month Follow- } \\
\text { up or Followed } \\
(n=270)\end{array}$ & $\begin{array}{c}\text { Loss-to-Follow-up } \\
\text { or Not- Followed } \\
(n=161)\end{array}$ & $p$-value \\
\hline Age $(M \pm S D)$ & $41.56 \pm 12.39$ & $42.58 \pm 12.82$ & $39.86 \pm 11.48$ & .023 \\
\hline $\begin{array}{l}\text { Education }(M \pm S D) \\
\text { Gender } n(\%)\end{array}$ & $8.45+3.39$ & $9.19 \pm 3.10$ & $7.37 \pm 3.57$ & $\begin{array}{l}.000 \\
.001\end{array}$ \\
\hline Female & $119(27.6 \%)$ & $90(33 \%)$ & $30(19 \%)$ & \\
\hline Male & $312(72.4 \%)$ & $180(67 \%)$ & $131(81 \%)$ & \\
\hline Marital Status $n(\%)$ & & & & .176 \\
\hline Single & $350(81.2 \%)$ & $155(57 \%)$ & $83(57 \%)$ & \\
\hline Married & $81(18.8 \%)$ & $115(43 \%)$ & $78(43 \%)$ & \\
\hline Ethnicity $n(\%)$ & & & & .001 \\
\hline Hispanic & $270(62.6 \%)$ & $142(52 \%)$ & $129(80 \%)$ & \\
\hline African American & $161(37.4 \%)$ & $128(48 \%)$ & $32(20 \%)$ & \\
\hline Prior STI Diagnosis $n(\%)$ & $102(23.7 \%)$ & $27(17 \%)$ & $75(28 \%)$ & .009 \\
\hline Number of $\mathrm{AC}(M \pm S D)$ & $103.77 \pm 138.32$ & $54.42 \pm 71.83$ & $101.09 \pm 140.44$ & .137 \\
\hline $\mathrm{MU}(M \pm S D)$ & $9.27 \pm 18.94$ & $6.60 \pm 15.91$ & $10.72 \pm 31.98$ & .122 \\
\hline $\mathrm{CU}(M \pm S D)$ & $15.61 \pm 34.24$ & $4.01 \pm 23.34$ & $27.55 \pm 85.91$ & .001 \\
\hline
\end{tabular}

Note: STI=Sexually Transmitted Infection; SP= Sexual Partners; UVA= Unprotected Vaginal Acts; $\mathrm{AC}=$ Alcohol Consumption; $\mathrm{MU}=$ Marijuana Use; $\mathrm{CU}=$ Crack Use. All Sexual acts and consumption of alcohol and illegal substances refer to the last 30 days.

\section{Ethnic Identity and Alcohol and Other Drugs}

Chi square analysis and independent $t$-tests were executed to provide a clearer picture of the association between ethnic identity subscales, demographic variables, and alcohol and drug use. The same analyses were conducted for changes in ethnic identity subscales with demographic variables and alcohol and drug use. Table 1.2, found below, describes high and low scores of ethnic identity explore and ethnic identity belonging by demographic variables and alcohol, marijuana, and crack use. Table 1.2 displays positive (increase) change in ethnic identity explore and ethnic identity belonging and no change and/or negative (decrease) change in ethnic identity explore and ethnic identity belonging scores for the 6-month follow-up assessment by demographic variables and alcohol, crack, and marijuana use. 
At baseline $(n=431)$, females had significantly lower scores in ethnic identity explore than males $\left(\chi^{2}=3.98, p=.029\right)$. Those participants who had resided in Immokalee for more than five years had significantly lower scores in ethnic identity explore than participants who had relocated there more recently $\left(\chi^{2}=6.87, p=.006\right)$. Regarding ethnic identity belonging, participants who spoke English had significantly lower scores in ethnic identity belonging than Spanish speaking participants $\left(\chi^{2}=3.57, p\right.$ $=.038)$. Participants in the low ethnic identity explore group reported a higher consumption of alcohol in the last month $(t=3.14, p=.002)$ than those in the high ethnic identify explore group.

The findings suggest that those with lower ethnic identity belonging score at baseline had a higher average of alcohol consumption, marijuana use, and crack use than participants with higher ethnic identity belonging; however, none of these differences were found to be statistically significant (Table 1.2). Those participants with a lower ethnic identity explore score at baseline had a higher average of alcohol consumption, marijuana use, and crack use than participants with higher scores of ethnic identity explore. However, the only statistically significant finding was those participants with higher ethnic identity explore were found to consume less alcohol than participants with lower ethnic identity explore at baseline $(t=3.138, p=.002)$. 
Table $1.2(2)$

Differences in Ethnic Identity Explore and Ethnic Identity Belonging by demographics and Substance Use ( $n=431 ; 6$ months: $n=270)$

\begin{tabular}{|c|c|c|c|c|c|c|c|c|}
\hline Demographics & $\begin{array}{l}\text { High EI } \\
\text { explore }\end{array}$ & Low EI explore & $\begin{array}{c}\text { High EI } \\
\text { belonging }\end{array}$ & $\begin{array}{c}\text { Low EI } \\
\text { belonging }\end{array}$ & $\begin{array}{c}\text { Positive EI } \\
\text { explore } \Delta \text { at } \\
6 \text { months }\end{array}$ & $\begin{array}{l}\text { No/negative } \\
\text { EI explore } \Delta \\
\text { at } 6 \text { months }\end{array}$ & $\begin{array}{c}\text { Positive EI } \\
\text { belonging } \Delta \text { at } \\
6 \text { months }\end{array}$ & $\begin{array}{l}\text { No/negative } \\
\text { EI belonging } \\
\Delta \text { at } 6 \text { months }\end{array}$ \\
\hline Age $(M+S D)$ & $42.81 \pm 12.33$ & $40.55 \pm 12.37$ & $43.13 \pm 12.83$ & $40.83 \pm 12.13$ & $41.43 \pm 12.58$ & $43.49 \pm 12.89$ & $42.65 \pm 12.35$ & $42.33 \pm 13.28$ \\
\hline Education $(M+S D)$ & $8.29 \pm 3.54$ & $8.58 \pm 3.26$ & $8.34+3.27$ & $8.51 \pm 3.44$ & $8.82 \pm 3.27$ & $9.53+2.91$ & $8.99+3.15$ & $9.43+3.04$ \\
\hline \multicolumn{9}{|l|}{ Marital status $n(\%)$} \\
\hline Single & $152,56.6 \%$ & $198,43.4 \%$ & $113,32.3 \%$ & $237,67.7 \%$ & $84,54.2 \%$ & $71,45.8 \%$ & $76,49.0 \%$ & $79,51.0 \%$ \\
\hline Married & $41,50.6 \%$ & $40,49.4 \%$ & $24,29.6 \%$ & $57,70.4 \%$ & $46,40 \%$ & $69,60 \%$ & $57,49.6 \%$ & $58,50.4 \%$ \\
\hline \multicolumn{9}{|l|}{ Language $n(\%)$} \\
\hline English & $115,43.1 \%$ & $152,56.9 \%$ & $76,28.5 \% *$ & $191,71.5 \% *$ & $85,42.9 \%$ & $113,57.1 \%$ & $88,44.4 \%$ & $110,55.6 \%$ \\
\hline Spanish & $78,47.6 \%$ & $86,52.4 \%$ & $61,37.1 \% *$ & $103,62.9 \% *$ & $45,62.5 \%$ & $27,37.5 \%$ & $45,62.5 \%$ & $27,37.5 \%$ \\
\hline \multicolumn{9}{|l|}{ Gender $n(\%)$} \\
\hline Male & $149,47.8 \%$ & $163,52.2 \% *$ & $105,33.7 \%$ & $207,66.3 \%$ & $95,52.8 \%$ & $85,47.2 \%$ & $96,53.3 \%$ & $84,46.7 \%$ \\
\hline Female & $44,37.0 \%$ & $75,63.0 \% *$ & $32,26.9 \%$ & $87,73.1 \%$ & $35,38.9 \%$ & $55,61.1 \%$ & $37,41.1 \%$ & $53,58.9 \%$ \\
\hline \multicolumn{9}{|l|}{ Length of Stay $n(\%)$} \\
\hline Longtimers ( $>5$ yrs) & $179,59 \%$ & $125,41 \%$ & $95,31 \%$ & $209,69 \%$ & $94,43 \% *$ & $124,57 \% *$ & $104,48 \%$ & $114,52 \%$ \\
\hline Newcomers ( $\leq 5$ yrs) & $59,46 \%$ & $68,54 \%$ & $42,33 \%$ & $85,67 \%$ & $37,73 \% *$ & $16,27 \% *$ & $29,57 \%$ & $23,43 \%$ \\
\hline \multicolumn{9}{|l|}{ Ethnicity $n(\%)$} \\
\hline Hispanic & $124,46.0 \%$ & $146,54.0 \%$ & $88,32.6 \%$ & $182,67.4 \%$ & $75,52.8 \%$ & $67,47.2 \%$ & $74,52.1 \%$ & $68,47.9 \%$ \\
\hline African American & $69,42.9 \%$ & $92,57.1 \%$ & $49,30.4 \%$ & $112,69.6 \%$ & $55,42.9 \%$ & $73,57.1 \%$ & $59,46.1 \%$ & $69,53.8 \%$ \\
\hline Alcohol Use $(M+S D)$ & $80.35+107.32 *$ & $122.06+156.17^{*}$ & $106.06+129.33$ & $\frac{102.70+142}{52}$ & $26.58+87.26$ & $-.26 \pm 72.28$ & $23.28+93.46$ & $2.36+65.00$ \\
\hline Crack Use $(M+S D)$ & $14.22+23.50$ & $16.69+40.73$ & $12.12+16.89$ & $17.23+39.75$ & $2.55 \pm 33.44$ & $1.74+11.49$ & $4.39+32.69$ & $-.06 \pm 12.21$ \\
\hline Marijuana Use $(M+S D)$ & $8.80 \pm 21.52$ & $9.64+16.69$ & $8.26 \pm 11.67$ & $9.74+21.51$ & $1.27 \pm 18.51$ & $-1.48 \pm 18.48$ & $.27 \pm 19.60$ & $-.57 \pm 17.46$ \\
\hline
\end{tabular}

Note: EI = ethnic identity. Ethnic identity explore was divided into a dichotomous dummy variable based on the mean.

${ }^{*} p<.05$. Ethnic identity belonging and ethnic identity explore were divided into high and low based on the mean of the ethnic identity component used. 


\section{Alcohol and drug risk behaviors}

The participants consumed crack, on average, about 4.01 times in the last month $(n=57 ; S D=23.336)$, while marijuana was consumed on average about 6.60 times in the last 30 days $(n=109 ; S D=15.907)$. Hispanics were found to consume significantly more alcoholic drinks over the last 30 days (Hispanics: $M=16.56, S D=21.751$; African Americans: $M=9.77, S D=3.177 ; t=3.055, p=.002$ ) at baseline (Table 1.1).

Ethnic Identity and Alcohol and Other Drugs

Only one component of ethnic identity, ethnic identity explore, was a statistically significant predictor of alcohol use at baseline and reductions in alcohol use over 6 months. The variance explained was low which limits the effect of the impact ethnic identity explore may have on alcohol overall. Table 1.3 displays the unstandardized regression coefficients $(B)$, standard error, and standardized regression coefficients $(B)$ for ethnic identity subscales, ethnic identity explore and ethnic identity belonging, and the demographic variables for both blocks of the regression model. The linear regression for alcohol use was found to be significant in both blocks, final block: $F(7,423)=7.113$, $p<.001 . R^{2}$ for the model was .105 , and the adjusted $R^{2}$ was .091 . Ethnic identity explore was found to be a significant predictor of alcohol use $(\beta=-.171, t=-2.999, p=.003)$, and an inverse relationship was found between the variables. Gender $(\beta=-.175, t=-3.311, p$ $=.001)$, education $(\beta=-.116, t=-1.974, p=.049)$, and language $(\beta=.196, t=1.953, p=$ .050) were significant contributing predictors of alcohol use. The results suggested that males, participants with lower levels of education, those with lower levels of ethnic identity, and those who spoke Spanish, were likely to consume more alcohol in the last 30 days than their representative counterparts. 
The next step taken was to explore the relationship between changes in ethnic identity explore and ethnic identity belonging with changes in alcohol consumption over the 6 months. Ethnic identity explore change and length of stay were the two significant predictors for the change in number of alcoholic drinks. Regression analysis revealed that the final model significantly predicted alcohol consumption change, $F(5,265)=5.853, p$ $=.001 . R^{2}$ for the final model was .100 , and the adjusted $R^{2}$ was .083 . Table 1.3 displays the unstandardized regression coefficients $(B)$, standard error, and standardized regression coefficients $(B)$ for changes in ethnic identity subscales, ethnic identity explore and ethnic identity belonging, and the demographic variables for both blocks of the regression model. Changes in ethnic identity explore were found to be associated with changes in alcohol consumption in the last 30 days suggesting that an increase in a participant's ethnic identity explore was associated with a reduction in the number of drinks consumed by the participant between baseline and 6-month follow-up, $(\beta=-.224, t$ $=3.419, p=.001)$. The only demographic variable that was a significant predictor for change in alcohol use was length of stay, $(\beta=.152, t=2.581, p=.010)$. The results suggest that participants who had been in Immokalee for more than 5 years and those who increased their ethnic identity explore were more likely to reduce their number of alcoholic drinks in the last 30 days compared to those who were newer to the area or either had negative/no change in their ethnic identity score.

Ethnic identity explore and ethnic identity belonging were found to significantly predict the number of times crack was used in the last 30 days (ethnic identity explore: $\beta$ $=.170, t=2.470, p=.014$; ethnic identity belonging: $\beta=-.235, t=-3.329, p=.001)$. An inverse relationship was found only between ethnic identity belonging and crack use. 
Ethnic identity explore had a positive relationship with crack use. Table 1.3 displays the unstandardized regression coefficients $(B)$, standard error, and standardized regression coefficients $(B)$ for changes in ethnic identity subscales, ethnic identity explore and ethnic identity belonging, and the demographic variables for both blocks of the regression model. The multiple linear regression model executed examining crack use was significant in both blocks, the final block was significant: $F(3,427)=3.967, p=.008 . R^{2}$ for the model was .027 , and the adjusted $R^{2}$ was .020 . Ethnic identity subscales were the only significant predictors of crack use, ethnic identity explore $(\beta=.145, t=2.470, p=$ $.014)$ and ethnic identity belonging $(\beta=-.196, t=-3.329, p=.001)$. The results suggest that those with lower levels of ethnic identity belonging are likely to consume more crack than their counterparts, while higher levels of ethnic identity explore were associated with higher levels of crack use in the last 30 days. It is important to note that the amount of explained variance found for the regressions was small which may limit the role ethnic identity belonging and ethnic identity explore may have on crack use. No associations were found when the relationships between changes in crack use and changes in ethnic identity belonging and ethnic identity explore were examined. Marijuana use was assessed in the same fashion to detect any relationship with ethnic identity belonging and ethnic identity explore; however, no relationships were found. 
Table $1.3(3)$

Regression Analysis Summary for Ethnic Identity Predicting Alcohol and Drug use at Baseline $(n=431)$ and over 6 months $(n=270)$

\begin{tabular}{|c|c|c|c|c|c|c|c|c|c|}
\hline \multirow{2}{*}{$\begin{array}{l}\text { Outcome } \\
\text { Variable } \\
\end{array}$} & \multirow{2}{*}{$\begin{array}{c}\text { Independent } \\
\text { Variables }\end{array}$} & \multicolumn{4}{|c|}{ Block 1} & \multicolumn{4}{|c|}{ Block 2} \\
\hline & & $R^{2}$ & $B$ & $S E$ & $\beta$ & $R^{2}$ & $B$ & $S E$ & $\beta$ \\
\hline \multirow[t]{8}{*}{ Alcohol } & & .086 & & & & .105 & & & \\
\hline & Language & & .319 & .151 & $212^{*}$ & & .294 & .150 & $.196^{*}$ \\
\hline & Gender & & -.271 & .087 & $-.166^{*}$ & & -.285 & .086 & $-.175^{*}$ \\
\hline & Education & & -.023 & 013 & -.105 & & -.025 & .013 & $-.116^{*}$ \\
\hline & Ethnicity & & .124 & .091 & .082 & & .120 & .090 & .080 \\
\hline & Country of Birth & & .290 & .152 & .194 & & .272 & .151 & .182 \\
\hline & EI Explore & & & & & & -.284 & .095 & $-.171 *$ \\
\hline & EI Belonging & & & & & & .171 & .098 & .100 \\
\hline \multirow[t]{4}{*}{ Crack } & & .001 & & & & .027 & & & \\
\hline & Gender & & .034 & .055 & .030 & & .035 & .055 & .031 \\
\hline & EI Explore & & & & & & .170 & .069 & $.145^{*}$ \\
\hline & EI Belonging & & & & & & -.235 & .071 & $-.196 *$ \\
\hline \multirow[t]{8}{*}{ SRAE-IR } & & .075 & & & & .105 & & & \\
\hline & Language & & .151 & 206 & .073 & & .137 & 203 & .066 \\
\hline & Gender & & -.352 & .120 & $-.157 *$ & & -.336 & .119 & $-.150 *$ \\
\hline & Ethnicity & & .346 & .126 & $.167^{*}$ & & .363 & .124 & $.175^{*}$ \\
\hline & Length of Stay & & -.325 & .119 & $-.148^{*}$ & & -.306 & .118 & $-.139 *$ \\
\hline & Country of Birth & & .363 & .216 & .176 & & .385 & .213 & .187 \\
\hline & EI Explore & & & & & & .262 & .131 & $.114^{*}$ \\
\hline & EI Belonging & & & & & & .194 & .134 & .082 \\
\hline \multirow[t]{6}{*}{ Alcohol $\Delta$} & & .038 & & & & .100 & & & \\
\hline & Length of Stay & & 24.270 & 11.961 & $.122^{*}$ & & 30.204 & $\begin{array}{c}11.70 \\
0\end{array}$ & $.152^{*}$ \\
\hline & Marital Status & & -23.575 & 9.830 & $-.145^{*}$ & & -16.546 & 9.703 & -.101 \\
\hline & Intervention & & 2.840 & 9.749 & .018 & & 1.501 & 9.538 & .009 \\
\hline & EI Explore $\Delta$ & & & & & & 29.948 & 8.760 & $.224 *$ \\
\hline & EI Belonging $\Delta$ & & & & & & 6.855 & 7.674 & .058 \\
\hline \multirow[t]{5}{*}{ SRAE-IR $\Delta$} & & .065 & & & & .190 & & & \\
\hline & Length of Stay & & -.170 & .162 & -.064 & & -.207 & .161 & -.078 \\
\hline & Intervention & & .013 & .132 & .006 & & -.005 & .131 & -.003 \\
\hline & EI Explore $\Delta$ & & & & & & -.351 & .119 & $-.197 *$ \\
\hline & EI Belonging $\Delta$ & & & & & & 147 & .106 & .093 \\
\hline
\end{tabular}

Note: $\mathrm{EI}=$ ethnic identity; Demo = demographics; $\mathrm{AC}=$ Alcohol Consumption; $\mathrm{CU}=$ Crack use; Adj. = adjusted; SRAE-IR = Sex Related Alcohol Expectancy MeasureIncreased Risk. All substance use behaviors refer to the last 30 days. $* p \leq 0.05$

Only one component of the SRAE subscales, SRAE-Increased Risk, was found to have a statistically significant association with ethnic identity explore at baseline and at 6-month follow-up; no relationship was found with SRAE as a total measure or any other 
SRAE subscales with ethnic identity belonging. An interesting finding was made regarding SRAE-Increased Risk. The findings suggest that a direct relationship exists between ethnic identity explore and SRAE-Increased Risk, only at baseline. The results, at baseline, revealed that ethnic identity explore was a significant predictor for SRAEIncreased Risk score, $(\beta=.262, t=1.999, p=.046)$, illustrating a direct relationship with SRAE-Increased Risk score and ethnic identity score of the participant. The linear regression model for SRAE-Increased Risk was found to be significant in both blocks, the final block was significant: $F(7,423)=7.074, p<.001$. Table 1.3, found above, displays the unstandardized regression coefficients $(B)$, standard error, and standardized regression coefficients $(B)$ for the ethnic identity subscales and the demographic variables for each block of the regression. Gender $(\beta=-.150, t=-2.833, p=.005)$, ethnicity $(\beta=$ $.175, t=-2.931, p=.004)$, and length of stay in Immokalee $(\beta=-.139, t=-2.599, p=$ .010) were found to significantly predict scores on the SRAE-Increased Risk subscale. The results suggest that those with higher scores on ethnic identity explore had more positive beliefs about the effects of alcohol on increasing the risk in sexual encounters. Male participants, Hispanic participants, and those participants who were recent residents in Immokalee were more likely to believe in the positive effects of alcohol consumption on engaging in risky sexual behaviors.

After SRAE-Increased Risk was analyzed at baseline, the same analysis was conducted for changes in SRAE-Increased Risk and changes in ethnic identity explore and ethnic identity belonging. Ethnic identity explore change was the only significant predictor of SRAE-Increased Risk change, $(\beta=-.197, t=-2.953, p=.003)$. Regression analysis revealed that the final model significantly predicted SRAE-Increased Risk 
change, $F(4,265)=2.475, p=.045 . R^{2}$ for the final model was .036 , and the adjusted $R^{2}$ was .021. Table 1.3, found above, displays the unstandardized regression coefficients $(B)$, standard error, and standardized regression coefficients $(B)$ for changes in ethnic identity explore and ethnic identity belonging as well as the demographic variables in both blocks. Length of stay and intervention were the two variables included in the first block. Ethnic identity explore and ethnic identity belonging were placed in the second block of the regression model. The results suggest that individuals who had positive changes in their ethnic identity explore scores were more likely to reduce their SRAE-Increased Risk score. This suggests that ethnic identity explore may have a protective effect on participants' reducing their positive beliefs in the power of alcohol to improve their sexual encounters.

Changes in Ethnic Identity

Regarding ethnic identity change over 6 months, participants who increased or demonstrated a positive change in their ethnic identity scores over the 6-month follow-up period reduced their level of alcohol use, marijuana use, and crack use more than participants who illustrated no change or a decrease in their ethnic identity explore score or ethnic identity belonging score (found in Table 1.2). Of the independent $t$-tests conducted on changes of ethnic identity belonging and ethnic identity explore, only participants with increases or positive changes in ethnic identity explore $(t=-2.76, p=$ $.007)$ and ethnic identity belonging $(t=-2.14, p=.033)$ were associated with a statistically significant lower number of alcoholic drinks, about 24 drinks, than participants with decreases or no change in ethnic identity explore and ethnic identity 
belonging. Such findings posit the possibility that ethnic identity explore may have a protective influence in relation to alcohol and drug use among migrant workers.

A Repeated Measures Analysis of Covariance (RMANCOVA) was conducted to assess if any short-term changes in ethnic identity as a total measure; ethnic identity explore and ethnic identity belonging actually took place over the 6-month follow-up period. Ethnic identity as a total measure, ethnic identity explore, and ethnic identity belonging significantly changed over the 6-month assessment period (Table 1.4). After controlling for intervention assignment, language, marital status, education, gender, country of origin, and length of stay, the time effect for ethnic identity total and ethnic identity belonging was not significant $(F(1,262)=2.45, p=.119)$ and $F(1,262)=.826, p$ $=.364)$ respectively. However, the time effect for ethnic identity explore was found to be statistically significant after controlling for covariates, $(F(1,262)=4.02, p=.046)$, but the variance explained was very small. The changes in all three analyses were small, but were found to be statistically significant.

Table 1.4 (4)

Repeated Measures ANCOVA for Ethnic Identity $(n=270)$

\begin{tabular}{|c|c|c|c|c|}
\hline & Baseline & 6 Months & $\begin{array}{c}F \\
\text { time effect only }\end{array}$ & $\begin{array}{c}F \\
\text { covariates } \\
\text { included }\end{array}$ \\
\hline Ethnic Identity Total & & & $12.340^{*}$ & 2.447 \\
\hline$M$ & 2.8639 & 2.9784 & & \\
\hline$S D$ & .37697 & .40347 & & \\
\hline EI Belonging & & & $14.075^{*}$ & .826 \\
\hline \multirow{4}{*}{ EI Explore } & 3.0476 & 3.1937 & & \\
\hline & .42224 & .50141 & & $410 *$ \\
\hline & 2.6067 & 2.6770 & & \\
\hline & .43125 & .45788 & & \\
\hline
\end{tabular}

Note: Covariates: language, marital status, country of origin, ethnicity, gender, intervention assignment, length of stay.

$* p \leq 0.05$ 


\section{DISCUSSION}

Ethnic Identity: Alcohol and Other Drugs

Several important findings are worthy of discussion regarding the role of ethnic identity with alcohol use, drug use, and beliefs of the effects of alcohol on sexual encounters, especially since migrant workers have been categorized as a highly susceptible group to HIV transmission (Organista et al., 2004; HJKFF, 2007). Ethnic identity explore was the subscale that illustrated an inverse relationship with migrant workers with riskier levels of alcohol. Participants with lower ethnic identity explore had higher alcohol use. Similar findings were illustrated for changes in ethnic identity and changes in alcohol consumption, possibly demonstrating a protective role. Previous research on ethnic identity and alcohol has shown that an inverse relationship exists between ethnic identity, as a full measure, and alcohol and/or drug use, as suggested by the findings of this study. Higher levels of ethnic identity were associated with lower levels of substance use and negative emotion in a study by Beadnell et al. (2003). This study examined the extent that baseline levels of ethnic identity offset negative psychobehavioral characteristics in African American adolescents, ultimately affecting their substance abuse practices in a positive manner (Beadnell et al., 2003).

The concept of ethnic identity explore, being assessed as a separate ethnic identity component, is novel regarding substance use in general. Higher levels of alcohol consumption have been associated with decreased protective behaviors and increased levels of disinhibiting behaviors which were illustrated in the study by Cooper (2002) and another study by Keine, Barta, Tenen, \& Armeli (2009). This study's results suggest that ethnic identity explore may carry protective traits regarding alcohol consumption since 
higher ethnic identity explore is associated with lower consumption of alcohol at baseline and over a 6-month time period. The literature on alcohol use and ethnic identity is almost non-existent. The studies that look into this topic note that adolescents who consume higher levels of alcohol or have higher levels of substance abuse tend to have lower levels of ethnic pride as well as ethnic and self-identity among Hispanics and African Americans (Beadnell et al., 2003; Marsiglia, Kulis, Hecht, \& Sills, 2004; Townsend \& Belgrave, 2000). Similar relationships may be found in migrant worker communities with the same type of ethnic group breakdown. It is important to mention that the findings of the aforementioned studies don't assess changes over time; the results are from a cross-sectional study only. The current study is breaking new ground in assessing changes over time.

Sex Related Alcohol Expectancy (SRAE) measure has been suggested to have a strong relationship with alcohol consumption and alcohol's effects on sexual encounters (Kalichman, Simbayi, Jooste, Vermaak, \& Cain, 2008; Leigh, 1990). Positive alcohol expectancies have been suggested to be associated with higher levels of alcohol consumption in addition to engaging in sexual behaviors. Sexual behaviors under the influence of alcohol may lead to unprotected sexual acts, increasing the risk of HIV (Brown \& Van Hook, 2006; Cooper, 2002; Seth, Wingood, DiClemente, \& Robinson, 2011; Zamboanga, 2005). The alcohol expectancy measure, increased riskiness, was the only alcohol expectancy component to have a statistically significant relationship with ethnic identity explore.

Our findings with respect to the increased riskiness alcohol expectancy measure illustrated a direct relationship with ethnic identity explore, which was unexpected. 
Migrant workers with higher scores on their beliefs that alcohol increases risky sexual behavior also revealed higher scores on their ethnic identity explore. It is possible that participants with higher ethnic identity explore recognize the riskiness involved with high levels of alcohol abuse and choose not to consume alcohol for those underlying factors, which may be a protective trait in itself. No literature was found that addresses ethnic identity and its relationship with alcohol expectancy measures. The link between alcohol expectancies, alcohol use, and sexual behaviors allows the conclusion that the negative relationship demonstrated between ethnic identity explore and SRAE-Increased Risk may be protective. Another concept assessed in this study is the changes in substance use across time in conjunction with the predictors (ethnic identity explore and ethnic identity belonging); this has not been explored in the literature.

Literature on drug use has suggested that ethnic identity may play a protective role, similar to the relationship found between crack use and ethnic identity belonging (Brook \& Pahl, 2005). However, a finding that must be further explored is the relationship found between ethnic identity explore and crack use revealing that the higher the participant's ethnic identity explore score, the higher their crack use. This analysis suggests a relationship that cannot be explained by the current literature on ethnic identity, since research on ethnic identity explore is sparse.

It is important to note that there was a difference found between participants at baseline and those participants that were followed at 6-month follow-up. Lost participants were less educated, younger, and engaged in riskier levels of alcohol use, crack use, and marijuana use compared to those participating at the 6-month follow-up. The loss of participants that engaged in risky levels of substance use may have affected our findings 
since the 6-month sample analyzed involved those that engaged in safer levels of use. Ethnic identity explore change was found to be a statistically significant predictor for alcohol consumption change, which illustrates that a reduction in alcohol consumption (fewer number of drinks during the last 30 days from baseline to 6 months) coincided with an increase in ethnic identity. The exploration aspect of ethnic identity is essential for ethnic identity development and formation; this phase entails talking to others about their ethnic group and discussing practices and traditions within this group (Phinney \& Ong, 2007). It must be noted that the ethnic identity subscale that has been examined in previous research is ethnic identity belonging. Since migrant workers are a highly mobile group, the belonging aspect of ethnic identity may be placed in question. Even though migrant workers are mobile, their contact with other migrant workers might highlight the differences between their ethnic group and the U.S. culture, which may lead them to seek out more information about their own ethnic culture.

Relating to the Identity Based Motivation theory, only 2 out of the 3 baseline outcome behavior variables tested can be categorized as identity-infused behaviors. These baseline outcome variables are alcohol consumption and crack use. The alcohol expectancy measures were not applicable to the theory used to guide this study because it is not an actual behavior. However, the findings fit along the lines of risky beliefs of alcohol which have been found to be linked to actual alcohol consumption. Over the 6month assessment period, only 1 of the 3 outcome behavior change variables assessed can be categorized as an identity-infused behavior, alcohol consumption change. Relating to change, SRAE-Increased Risk was found to be inversely associated with ethnic identity explore, which may be a protective trait. These are categorized as protective 
identity-infused behaviors since the higher the ethnic identity, the lower the HIV risk regarding these outcome variables.

\section{Ethnic Identity: Changes over Time}

Ethnic identity was found to change over time for ethnic identity as a total measure and for ethnic identity belonging and ethnic identity explore. Ethnic identity changes over time have been reported in previous studies targeting adolescent children. However, the literature on such changes in ethnic identity among adults is very sparse. Changes in ethnic identity may be a result of changes in one's environment, such as changes in family cohesion or changes in ethnic peers (Kiang, Witkow, Baldelomar, \& Fuligni, 2010). The literature which discusses ethnic identity among adults concludes that changes in ethnic identity may occur in adults depending on specific circumstances (Kiang, Witkow, Baldelomar, \& Fuligni, 2010; Phinney, Horencyzk, Liebkind, \& Vedder, 2001). The specific circumstances mentioned above entail life changing circumstances or changes in environment, or both combined which may occur and impact individuals' self-identity as well as their ethnic identity. Such changes may lead to identity reformulation, which also causes alterations in one's ethnic identity (Torres et al., 2012).

Although this study found an initial change in ethnic identity over time, this change became non-significant with the inclusion of demographic covariates. The trend toward change in this sample, however, suggests that there may be commonalities between adolescents' ethnic identity change and adult ethnic identity change. Both groups experience instability which may influence ethnic identity. Migrant workers have an unstable environment due to employment or migration issues or the psychosocial 
conflicts they face as they adjust to their roles in a new environment (Marcia, 1966).

Among adolescents, instability is due to their physical and psychological changes as well as the exploration of their self-identity and ethnic identity (Kiang, Witkow, Baldelomar, \& Fuligni, 2010). A study by Torres and colleagues (2012) concluded that ethnic identity changes in Latinos during adulthood occurred as a result of changes in the environment. This change in ethnic identity revolved around a reevaluation of identity and their attachment to their ethnic group. In the case of migrant workers and adolescents, instability may ultimately affect their ethnic identity. Migrant workers have unstable employment and very often must adapt to their changing environments. These factors may account for the initial changes seen in ethnic identity subscales, as well as the full scale, found in this migrant worker sample. It is important to note that the variance explained by the changes in ethnic identity was small for the results found. This may be due to the idea that more time is needed to examine the change in ethnic identity, especially since some of the migrant workers have lived in Immokalee for 5 years or more. The amount of time allotted to assess the change may not have been sufficient because of the various differences in length of time residing in Immokalee found in this migrant worker sample.

\section{Other Contributing Factors}

Other contributing predictors of alcohol consumption at baseline were marital status and length of stay. Such findings imply that single migrant workers, migrant workers who have been in the U.S. for 5 years or more, and participants who had increased ethnic identity explore scores were more likely to reduce their alcohol consumption for the last 30 days. The significant difference found in the amount of 
positive change in alcohol consumption among single migrant workers is unexpected. Such findings are inconsistent with the literature on alcohol consumption and marital status (Oesterle, Hawkins, \& Hill, 2011) which states that single individuals tend to be at higher risk of alcohol abuse than their married counterparts. However, the literature does not include any studies on the factors of length of stay and ethnic identity level.

The analyses reported suggest that increases in ethnic identity may have an impact in the reduction of alcohol consumption in the 6-month sample analyzed. Further studies are needed to determine the associations. These findings suggest a protective role of ethnic identity explore; however, the small amount of variability found in the models raises concerns regarding the benefits of ethnic identity indicators in future interventions.

\section{Limitations}

The limitations of the study must be noted. It would have been preferable if the sample were larger to increase the power of the analyses conducted. However, the sample size was sufficient based on the power analysis. The data reported were all self-reported, which may increase the recall bias. It is possible that in this high risk group of migrant workers, there may be an enhanced likelihood of inaccurate reporting. A minimal difference was found in ethnic identity from baseline to 6-month follow-up period. Such a minimal difference can be a result of error. Another limitation to this study is how the 161 participants lost to follow-up were clearly a riskier group than the participants followed in the 6-month follow-up sample. The inclusion of such high risk participants in our study may have yielded drastically different results. 


\section{Implications}

These findings are just the stepping stones toward understanding the role ethnic identity may play among migrant workers, especially the role of ethnic identity explore. Future studies on ethnic identity in migrant workers may be helpful; however, it must be noted that the variance explained for the results reported were very small which limits the actual effect that ethnic identity may have had. The role of ethnic identity needs to be further explored, over a longer period of time, among migrant worker communities in the U.S., especially since they are composed of minority groups that live with the stress of being in an unstable environment. 


\section{REFERENCES}

Albarrán, C. R., \& Nyamathi, A. (2011). HIV and Mexican migrant workers in the United States: a review applying the vulnerable populations conceptual model. The Journal of the Association of Nurses in AIDS Care, 22(3), 173-85. doi:10.1016/j.jana.2010.08.001

Altschul, I., Oyserman, D., \& Bybee, D. (2006). Racial-ethnic identity in midadolescence: content and change as predictors of academic achievement. Child Development, 77(5), 1155-69. doi:10.1111/j.1467-8624.2006.00926.x

Apostolopoulos, Y., Sonmez, S., Kronenfeld, J., Castillo, E., McLendon, L., \& Smith, D. (2006). STI/HIV risks for Mexican migrant laborers: exploratory ethnographies. Journal of Immigrant and Minority Health. 8(3), 291-302. doi: 10.1007/s10903-006-9334-2

Baliunas, D., Rehm, J., Irving, H., \& Shuper, P. (2010). Alcohol consumption and risk of incident human immunodeficiency virus infection: a meta-analysis. International Journal of Public Health, 55(3), 159-66. doi:10.1007/s00038-009-0095-x

Brook, J. S., Whiteman, M., Balka, E. N., Gursen, M. D., \& Win, P. T. (1998). Drug use among Puerto Ricans: Ethnic identity as a protective factor. Hispanic Journal of Behavioral Sciences, 20(2), 241-254. doi:10.1177/07399863980202007

Carroll, D. J, Samardick, R., Bernard, S., Gabbard S., Hernandez, T. (2005). Findings from the National Agricultural Workers Survey (NAWS) 2001-2001: A demographics and employment profile of United States farmworkers. (Research Report No. 9, U.S. Department of Labor).

Centers for Disease Control and Prevention (CDC). (2011). HIV in the United States: An Overview. Retrieved from:

http://www.cdc.gov/hiv/topics/surveillance/resources/factsheets/pdf/HIV-USoverview.pdf

Centers for Disease Control and Prevention (CDC). (2010a). HIV Surveillance Report, 2008. Retrieved from:

http://www.cdc.gov/hiv/surveillance/resources/reports/2008report/pdf/2008SurveillanceR eport.pdf

Centers of Disease Control and Prevention. (2010b). HIV transmission. Retrieved from http://www.cdc.gov/hiv/resources/qa/transmission.htm.

Centers for Disease Control and Prevention. (2009). HIV/AIDS in the United States. Retrieved from http://www.cdc.gov/hiv/resources/factsheets/us.htm. 
Cook, R. L., \& Clark, D. B. (2005). Is There an Association Between Alcohol Consumption and Sexually Transmitted Diseases? A Systematic Review. Sexually Transmitted Diseases, 32(3), 156-164. doi:10.1097/01.olq.0000151418.03899.97

Cooper, M. L. (2002). Alcohol use and risky sexual behavior among college students and youth: evaluating the evidence. Journal of Studies on Alcohol. Suppl. (14), 101-17. Retrieved from http://www.ncbi.nlm.nih.gov/pubmed/12022716

Dermen, K. H., \& Cooper, M. L. (1994). Sex-related alcohol expectancies among adolescents: I. Scale development. Psychology of Addictive Behaviors, 8(3), 152-160. doi:10.1037/0893-164X.8.3.152

Espinosa-Hernández, G., \& Lefkowitz, E. S. (2009). Sexual behaviors and attitudes and ethnic identity during college. Journal of Sex Research, 46(5), 471-482. doi:10.1080/00224490902829616

Faul, F., Erdfelder, E., Lang, A.-G., \& Buchner, A. (2007). G*Power 3: A flexible statistical power analysis for the social, behavioral, and biomedical sciences. Behavior Research Methods, 3(2)9, 175-191. doi: 10.3758/BF03193146

Fernandez, M. I., Collazo, J. B., Hernandez, N., Bowen, G. S., Varga, L. M., Vila, K. C., Arheart, K. L., \& Perrino, T. (2004). Predictors of HIV Risk Among Hispanic Farm Workers in South Florida: Women Are At Higher Risk Than Men. AIDS and Behavior, 8(2), 165-173. doi:10.1023/B:AIBE.0000030247.00140.62

Griffin, K. W., Bang, H., \& Botvin, G. J. (2010). Age of alcohol and marijuana use onset predicts weekly substance use and related psychosocial problems during young adulthood. Journal of Substance Abuse, 15(3), 174-183.

Gwyther, E. (1998). Migrant Farmworker Children: Health Status, Barriers to Care , and Nursing Innovations in Health Care Delivery. Journal of Pediatric Health Care, 12(2), 60-66. doi:10.1016/S0891-5245(98)90223-1

Hansen, E \& Donohoe, M. (2003). Health Issues of Migrant and Seasonal Farmworkers. Journal of Health Care for the Poor and Underserved, 14(2), 153-164. doi:10.1177/1049208903251513

Hiott, A. E., Gryzywacz, J. G., Davis, S. W., Quandt, S. A., \& Arcury, T. A. (2008). Migrant farmworker stress: mental health implications. The Journal of Rural Health, 24(1), 32-39. doi:10.1111/j.1748-0361.2008.00134.x

Hirsch, S., Higgins, J., Bentley, M. E., \& Nathanson, C. A. (2002). The social constructions of sexuality: marital infidelity and sexually transmitted disease-HIV risk in a Mexican migrant community. American Journal of Public Health, 92(8), 1227-1237. doi: 10.2105/AJPH.92.8.1227 
Kerpelman, J. L., Eryigit, S., \& Stephens, C. J. (2007). African American Adolescents' Future Education Orientation: Associations with Self-efficacy, Ethnic Identity, and Perceived Parental Support. Journal of Youth and Adolescence, 37(8), 997-1008. doi:10.1007/s10964-007-9201-7

Kim-Godwin, Y. S., \& Fox, J. A. (2009). Gender differences in intimate partner violence and alcohol use among Latino-migrant and seasonal farmworkers in rural southeastern North Carolina. Journal of Community Health Nursing, 26(3), 131-42. doi:10.1080/07370010903034474

Martínez-Donate, A. P., Rangel, M. G., Hovell, M. F., Santibáñez, J., Sipan, C.L., Izazola, J. A. (2005). HIV infection in mobile populations: the case of Mexican migrants to the United States. Revista Panamerican Salud Publica, 17(1), 26-9.

McCoy, H. V., Hlaing, W. M., Ergon-Rowe, E., Samuels, D., \& Malow, R. (2009). Lessons From the Fields: A Migrant HIV Prevention Project. Public Health Reports, 124(6), 790-6. Retrieved from http://www.ncbi.nlm.nih.gov/pmc/articles/PMC2773941/

Moreno, C. L., Morrill, A. C., \& El-Bassel, N. (2011). Sexual risk factors for HIV and violence among Puerto Rican women in New York City. Health \& Social Work.

Retrieved from http://www.ncbi.nlm.nih.gov/pubmed/21661298

Muñoz-Laboy, M., Hirsch, J. S., \& Quispe-Lazaro, A. (2009). Loneliness as a sexual risk factor for male Mexican migrant workers. American Journal of Public Health, 99(5), 802-10. doi:10.2105/AJPH.2007.122283

The National Agriculture Worker Survey (NAWS). (2010). Retrieved from http://www.doleta.gov/agworker/report9/chapter1.cfm

Organista P. B., Organista K. C., \& Soloff, P. R. (1998). Exploring AIDS related knowledge, attitudes, and behaviors of female migrant workers. Health and Social Work. 23(2):96-103.

Organista, K. C., \& Kubo, A. (2005). Pilot Survey of HIV Risk and Contextual Problems and Issues in Mexican/Latino Migrant Day Laborers. Journal of Immigrant Health, 7(4), 269-281. doi: 10.1007/s10903-005-5124-0

Organista, K.C. \& Organista, P.B. (1996). Migrant laborers and AIDS in the United States: review of the literature. AIDS Education and Prevention, 9, 83-93. doi: $10.1177 / 07399863960183008$

Organista, K.C., Carrillo, H., \& Ayala, G. (2004). HIV prevention with Mexican migrants: review, critique, and recommendations. Journal of Acquired Immune Deficiency Syndromes, 37, S227-39. 
Organista, K. C., Organista, P. B., Bola, J. R., Garcia de Alba J. E., \& Moran, M. A. C. (2000). Predictors of condom use in Mexican migrant laborers. American Journal of Community Psychology, 28, 245-265. doi: 0091-0562/00/0400-0245\$18.00/0

Oyserman, D., Yoder, N., \& Fryberg, S. A. (2007). Identity-Based Motivation and Health. Journal of Personality and Social Psychology, 93(6), 1011-1027.

Phinney, J. S. (1992). The Multigroup Ethnic Identity Measure: A New Scale for Use with Diverse Groups. Journal of Adolescent Research, 7(2), 156-176.

doi:10.1177/074355489272003

Phinney, J. S., Jacoby, B., \& Silva, C. (2007). Positive intergroup attitudes: The role of ethnic identity. International Journal of Behavioral Development, 31(5), 478-490. doi:10.1177/0165025407081466

Rangel, M. G., Martínez-Donate, A. P., Hovell, M. F., Santibáñez, J., Sipan, C. L., \& Izazola-Licea, J. a. (2006). Prevalence of risk factors for HIV infection among Mexican migrants and immigrants: probability survey in the North border of Mexico. Salud Pública de México, 48(1), 3-12. Retrieved from http://www.ncbi.nlm.nih.gov/pubmed/16555529

Rhodes, S. D., Bischoff, Ã. W. E., Burnell, J. M., Whalley, L. E., Walkup, M. P., Vallejos, Q. M., Quandt, S. A., et al. (2010). HIV and Sexually Transmitted Disease Risk among Male Hispanic / Latino Migrant Farmworkers in the Southeast: Findings from a Pilot CBPR Study. American Journal of Industrial Medicine, 53(10), 976-983. doi:10.1002/ajim.20807

Roberts, R. E., Phinney, J. S., Masse, L. C., Chen, Y. R., Roberts, C. R., \& Romero, A. (1999). The Structure of Ethnic Identity of Young Adolescents from Diverse Ethnocultural Groups. The Journal of Early Adolescence, 19(3), 301-322. doi:10.1177/0272431699019003001

Schwartz, S. J., Zamboanga, B. L., \& Jarvis, L. H. (2007). Ethnic identity and acculturation in Hispanic early adolescents: mediated relationships to academic grades, prosocial behaviors, and externalizing symptoms. Cultural Diversity \& Ethnic Minority Psychology, 13(4), 364-73. doi:10.1037/1099-9809.13.4.364

Shehadeh, N., McCoy, H. V., Rubens, M. B., Batra, A., Renfrew, R., \& Winter, K. (2011). The impact of ethnic identity on changes in high risk HIV Behaviors in sexually active migrant workers. Journal of Immigrant and Minority Health, 14(1), 100-106. doi: 10.1007/s10903-011-9466-5

Smith, E. P., Walker, K., Fields, L., Brookins, C. C., \& Seay, R. C. (1999). Ethnic identity and its Relationship to Self-Esteem, Perceived Self-Efficacy and Prosocial Attitudes in Early Adolescence. Journal of Adolescence, 22, 867-880. 
IBM Corporation. (2010). IBM SPSS Base 19.0 for Windows User's Guide. IBM Corporation, New York.

Susser, E., Desvarieux, M., \& Wittkowski, K. M. (1998). Reporting sexual risk behavior for HIV: a practical risk index and a method for improving risk indices. American Journal of Public Health, 88(4), 671-4. doi: 10.2105/AJPH.88.4.671

Turner, A. N., Miller, W. C., Padian, N. S., Kaufman, J. S., Behets, F. M., Chipato, T., Morrison, C. S. (2009). Unprotected sex following HIV testing among women in Uganda and Zimbabwe: short- and long-term comparisons with pre-test behaviour. International Journal of Epidemiology, 38(4), 997-1007. doi:10.1093/ije/dyp171

Utsey, S., Chae, M. H., Brown, C. F., \& Kelly, D. (2002). Effect of Ethnic Group Membership on Ethnic Identity, Race-Related Stress, and Quality of Life. Cultural Diversity and Ethnic Minority Psychology, 8(4), 366-377. doi: 10.1037/10999809.8.4.367

Villarejo, D., Mccurdy, S. A., Bade, B., Samuels, S., Lighthall, D., \& Williams III, D. (2010). The Health of California's Immigrant Hired Farmworkers. American Journal of Industrial Medicine, 53(4), 387-397. doi:10.1002/ajim.20796

Yoo, H. C. , Lee R. M. (2005). Ethnic identity and approach-type coping as moderators of the racial discrimination/well-being relation in Asian Americans. Journal of Counseling Psychology, 52(4), 497-506. doi:10.1037/0022-0167.52.4.497

Yi, H., Sandfort, T. G. M., \& Shidlo, A. (2010). Effects of disengagement coping with HIV risk on unprotected sex among HIV-negative gay men in New York City. Health Psychology, 29(2), 205-14. doi:10.1037/a0017786

Zamboanga, B. L., Raffaelli, M., \& Horton, N. J. (2006). Acculturation status and heavy alcohol use among Mexican American college students: investigating the moderating role of gender. Addictive Behaviors, 31(12), 2188-98. doi:10.1016/j.addbeh.2006.02 


\section{Chapter V}

\section{TRANSITION TO MANUSCRIPT 2}

This dissertation is written in a three-manuscript format. It was inevitable for some information to be excluded from the publishable article portion of each manuscript since some of the results did not directly relate to the proposed hypotheses. No relationship was found between short term changes in ethnic identity (the independent variable) and changes in HIV risk behavior/alcohol expectancy (the dependent variable). The dependent variables of the regressions that were not reported in detail in the manuscript were number of sexual partners at baseline, number of sexual partners without a condom at baseline, Vaginal Episodes Equivalent (VEE) change, number of partners in the last 30 days change, number of unprotected partners in the last 30 days change. These models were mentioned, but not described in detail in the publishable portions of the manuscript. These regressions are described in detail in Tables 2.4 through 2.9 found in Appendix 6. The following section is Manuscript 2 of this dissertation. The information most relevant to the proposed hypotheses was reported in the manuscript. 


\title{
MANUSCRIPT 2
}

\section{RISKY SEXUAL BEHAVIORS: THE IMPACT OF ETHNIC IDENTITY ON HIV RISK BEHAVIORS AMONG MIGRANT AND SEASONAL WORKERS}

\begin{abstract}
Background: In terms of HIV risk, migrant workers (MWs) are an often overlooked segment of the population. Many migrant workers in the U.S. engage in unsafe sexual behaviors, placing them at risk for contracting HIV. A number of studies indicate that the prevalence of HIV/AIDS is considerably higher among migrant workers. Ethnic identity has been posited to have an impact on engagement in risky sexual behaviors. This longitudinal study examines associations between baseline and short term changes in ethnic identity and high-risk sexual behaviors.
\end{abstract}

Methods: Baseline $(n=431)$ and 6-month assessment $(n=270)$ data were obtained from a larger ongoing HIV prevention intervention study conducted among migrant workers in Immokalee, Florida. The sample was composed of African American and Hispanic migrant workers. The ethnic identity measure used was the Multigroup Ethnic Identity Measure (MEIM). Repeated measures MANCOVA and multiple linear regressions were used to examine the relationship between ethnic identity and risky sexual behaviors.

Results: Ethnic identity belonging and ethnic identity explore, MEIM subscales, were examined at baseline and at the 6-month follow-up period. Ethnic identity explore was a statistically significant predictor of overall sexual risk $(F(8,422)=6.953, p<.001)$ and unprotected vaginal acts $(F(8,422)=8.444, p<.001)$ at baseline. However, ethnic identity had no bearing on changes in risky sexual behaviors. 
Conclusion: Findings suggest that ethnic identity explore may serve a protective role regarding sexual behaviors without condom use; however, these findings need to be further investigated since the effect size was minimal.

Key Words: Ethnic Identity, Migrant Workers, HIV Risk Behaviors, Sex Related Alcohol Expectancy 


\section{INTRODUCTION}

More than 9 million people in the U.S. are migrant workers (MWs), primarily concentrated in Texas, Florida, Washington, California, Oregon, and North Carolina (Carroll, Samardick, Bernard, Gabbard, \& Hernandez, 2005; Coppel, Dumont, \& Visco, 2001; Fitzgerald, Chakraborty, Shah, Khuder, \& Duggan, 2003; Organista, P.C.,

Organista, K.C., \& Soloff, 1998; Passel, Caps, \& Fix, 2004; Rhodes et al., 2010). Migrant workers are one of the most rapidly growing populations in the United States (U.S.). The place of origin for the majority of migrant workers is Mexico. Hispanics account for about $70 \%$ of the migrant workers in the U.S. (Villajero et al., 2010). This population has been significantly affected by HIV/AIDS (Coppel, Dumont, \& Visco, 2001; Villarejo, McCurdy, Bade, Samuels, Lighthall, \& Williams III, 2010). Among migrant workers in the U.S., the prevalence of HIV infection is between 2.6\% and 13\% (Organista et al., 1998; Organista et al., 2000; Organista et al., 2004).

The majority of migrant workers experience poverty (Albarran \& Nyamathi, 2011), social isolation (Muñoz-Laboy, Hirsch, \& Quispe-Lazaro, 2009), and lack of access to health care (Fitzgerald, Chakraborty, Shah, Khuder, \& Duggan, 2003). Research has suggested that migrant workers are at a heightened level of risk for HIV due to their engagement in a number of HIV risk behaviors, such as risky sexual behaviors and alcohol and substance use (Duke \& Carpintiero, 2009; Fernandez et al., 2004; Weatherby, McCoy, Bletzer, McCoy, Inciardi, McBride, \& Forney, 1997).

The challenges that migrant workers face because of migration increase their risk for contracting sexually transmitted infections (STIs) in general, especially HIV infection. Migrant workers are highly mobile which makes it challenging to properly 
monitor the incidence and prevalence rates of HIV in this vulnerable population (Villajero et al., 2010). The average income level of migrant workers tends to fall below the national poverty line (Villajero et al., 2010). Annually, single individuals make less than $\$ 7,500$, and five-person households make less than $\$ 10,000$ (Hansen \& Donohoe, 2003). On average, the highest educational level for migrant workers is seventh grade, and 20\% have been reported to be illiterate (Albarran \& Nyamathi, 2011; Denner, Organista, Dupree, \& Thrush, 2005; Hansen \& Donhoe, 2003). Little formal education and low income status contribute to the challenges that migrant workers face, making them more susceptible to negative health outcomes and HIV infection (Martinez-Donate et al., 2005; McCoy, Hlaing, Ergon-Rowe, Samuels, \& Malow, 2009).

A major problem affecting migrant workers is social isolation. Social isolation issues may be a result of language barriers and not knowing anyone in their new place of residence (Hirsch, Higgins, Bentley, \& Nathanson, 2002). Migrant workers often cope with their social isolation through seeking comfort in sexual intimacy by engaging in unsafe sexual practices (Apostolopoulos et al., 2006; Arcury \& Quandt, 2007; Henry J. Kaiser Family Foundation (HJKFF), 2007; Organista et al., 2004; Parker, Easton, \& Klein, 2000). Such risky sexual behaviors put migrant workers, as well as their families, at high risk of STI's and the HIV infection. These risky sexual behaviors include vaginal sex without a condom and multiple sex partners. Similar findings were found by MuñzLaboy et al. (2009) who concluded that risky sexual behaviors were associated with higher levels of loneliness in migrant workers (Muñoz-Laboy, Hirsch, \& Quispe-Lazaro, 2009).

In terms of health, studies have posited that ethnic identity may be associated with positive health behaviors like healthy lifestyle practices. Such healthy lifestyle practices 
include low consumption of alcohol, no/low substance use, and practicing HIV preventative behaviors (Brook, Whiteman, Balka, Win, \& Gursen, 1998; Nesdale, Rooney, \& Smith, 1997). Ethnic identity, as defined by Phinney, DuPont, Espinosa, Revil, and Sanders (1994), is “a feeling of belonging to one's group, a clear understanding of the meaning of one's membership, positive attitudes toward the group, familiarity with its history and culture and involvement in its practices" (p.169). Ethnic identity includes aspects of ethnic involvement such as language, behavior, values, and knowledge of ethnic group history (Persky \& Birman, 2005; Salgado de Snyder, Díaz Pérez, \& Maldonado, 2006). Phinney further defines the components of ethnic identity: ethnic identity exploration and ethnic identity belonging (Phinney, 1992). Ethnic identity "belonging," one of the components of ethnic identity included in Phinney's MEIM (one of the primary measures used in this study), includes ethnic pride, positive feelings toward one's ethnic group, and one's satisfaction with being a member of his/her ethnic group. Ethnic identity "explore," the second component, measures the extent to which individuals search for a sense of meaning in their ethnicity (Phinney, 1992). A moderating factor to changes in the development and exploration of ethnic identity in individuals is being a member of an ethnic minority group. In addition to being a minority, developmental levels of ethnic identity tend to be stronger in females than in males (Kiang, Witkow, Baldelomar, \& Fuligni, 2009).

Ethnic identity is distinguished from acculturation in that ethnic identity measures the level of attachment one has with his/her ethnic group while acculturation measures how one from an ethnic/minority group adapts to the mainstream culture. Many studies use acculturation and ethnic identification as one construct, even though it is crucial to recognize their differences. Acculturation is associated with changes in attitudes or 
beliefs relating to the mainstream or dominant culture. This concept is usually measured on a group level, while ethnic identification is assessed on the individual level. The majority of studies that measure acculturation fail to assess the multidimensional component of this construct (Abraido-Lanza et al., 2006). Ethnic identification measures the individual's emotional attachment (comfort level) and the engagement in cultural practices of his/her ethnic group (Phinney, 1992).

In terms of health, studies have posited that high ethnic identity may be associated with positive behaviors, such as healthy lifestyle practices which may consist of low consumption of alcohol, no substance use, and HIV prevention practices (Nesdale, Rooney, \& Smith, 1997; Brook, Whiteman, Balka, Win, \& Gursen, 1998). Although the literature on the relationship between ethnic identity and risky sexual behaviors is sparse, several studies have been conducted among differing ethnic groups. Some of the studies present a theory of ethnic identity as a protection against engagement in risky sexual and substance use behaviors. For example, in a study conducted by Beadnell and colleagues (2003), a "buffering" effect was shown among African American heterosexual females. There was a difference in risky sexual acts in the last 4 months depending on their level of ethnic identity: those with higher levels of ethnic identity had lower levels of risky sexual behaviors compared to the women with lower ethnic identity levels (Beadnell, Stielstra, Baker, Morrison, Knox, Gutierrez \& Doyle, 2003). A decline in identifying with one's ethnic group, which has been found among those who have been in the U.S. for long periods of time, has been linked to higher levels of depressive symptoms as well as lower self-esteem as was found in a study by Umana-Taylor and Updegraff (2007) of Latino adolescents. 
The limited literature suggests that ethnic identity changes over time, depending on the stage of development an individual is currently in and the life changing circumstances they may experience (Phinney, 1992, 2003). The only research found that addresses ethnic identity change in adulthood is a study by Torres et al. (2012). The findings of that study suggest that one's ethnic identity does change, and it may change in adulthood when experiencing life changing or stressful circumstances.

The current study focused on exploring 3 critical relationships: (1) does a relationship exist between ethnic identity belonging and/or ethnic identity explore with risky sexual behaviors (sexual risk, the number of vaginal sex acts without a condom, the total number of sexual partners, and the number of sexual partners without a condom), and (2) does ethnic identity change over a 6-month time period, and if so, (3) are these changes in ethnic identity belonging and ethnic identity explore associated with changes in risky sexual behaviors over the 6-month period?

\section{METHODS}

\section{Sample}

One geographic area that has been severely affected by the HIV epidemic, with elevated risks being detected in the migrant worker community, is South Florida (Fernandez, Collazo, Hernandez, Bowen, Varga, Vila, Arheart, \& Perrino, 2004). Data were obtained from a larger HIV risk reduction intervention study conducted in the South Florida agricultural area of Immokalee in Collier County, Florida. The study was implemented from 2005 through 2010, and focused on examining the effectiveness of two interventions: an enhanced/adapted cognitive behavioral program, PEER, compared to a health promotion program, HEALTHY, for producing long-term reductions in HIV risk and increased health behaviors among alcohol and other drug using migrant workers 
(McCoy, Hlaing, Ergon-Rowe, Samuels, \& Malow, 2009). The sample was composed of migrant workers recruited using a targeted sampling method. Inclusion criteria included the following: age 18 years or older; at least one episode of unprotected (without a condom) vaginal, anal, and/or oral sex in the past 90 days; alcohol or other drug intake in the past 90 days; and fluent in English and/or Spanish.

The present study used the data from baseline $(n=431)$ and the 6-month followup $(n=270)$. The study's definition of migrant workers conformed to the Public Health Services Act (1944) definition with two exceptions. The Public Health Services Act states that migrant workers are those individuals who are employed in agricultural labor, either seasonal or migratory, and live in temporary housing. The study criteria did not limit the definition of migrant workers to those who travel at least 75 miles or cross county lines to work. The study also included people who work or worked indirectly in agriculture, such as in packing houses. Seasonal workers were those migrant workers who moved from one place to another based on the season for the crops being picked.

The participants included in this study were recruited from "camps." In the community, "camps" referred to trailer parks, dormitory-style housing, apartment buildings, motels, duplexes, and neighborhoods of single/duplex housing in the immediate and surrounding areas. In the setting described above, potential participants were approached and asked a few questions to see if they complied with the eligibility criteria of the HIV prevention intervention project.

The theory that guided this study is known as the Identity Based Motivation Theory (Oyserman, Yoder, \& Fryberg, 2007). This theory focuses on how individuals from different ethnic/racial groups perceive accepted and unaccepted behaviors based on the perspective of a member of their ethnic/racial group. This link between ethnic 
identification and accepted and unaccepted behaviors is also known as identity-infused behaviors. This theory best described the link that exists between ethnic identity and unhealthy/high risk behaviors, in this case risky sexual behaviors. In many cases identityinfused behaviors are protective.

\section{Measures}

\section{Dependent Variables}

The dependent variables were self-reported and collected through the HIV/AIDS Risk Reduction Questionnaire (HRRQ), composed of 204 questions and covered 13 sections, utilized in the parent study. The sex behavior section of the questionnaire is where the dependent variable information was retrieved. Eight dependent variables, labeled as HIV risk behaviors, were examined and included in the regression models: Vaginal Episode Equivalent (VEE; defined below), VEE change, number of sexual partners, number of sexual partners change, number of sexual partners without a condom, number of sexual partners without a condom change, number of vaginal acts without a condom, and number of vaginal acts without a condom change. Change variables were created for each dependent variable to examine the impact of any changes (changes in scores or frequency of sexual behaviors) over the 6-month time period. The change variables were created by computing a new variable that calculated the difference between 6 months and baseline for the frequency of the behavior or score of the scales reported.

VEE weights oral, vaginal, and anal sex acts based on their differential risk. Compared to using individual specific sex acts (e.g., unprotected vaginal intercourse) as individual variables, this index has the advantage of providing a score reflecting subjects' overall sexual risk-taking behavior (Susser, Desvarieux, and Wittkowski, 1998). Higher 
VEE scores represent higher sexual risk. The formula for measuring VEE is: (number of unprotected vaginal sex acts $)+(2 \mathrm{x}$ number of unprotected anal sex acts $)+(0.01 \mathrm{x}$ number of unprotected oral sex acts) (Susser et al., 1998).

The number of sexual partners' variable sums the number of sexual partners in the last 30 days without reflecting the use or non-use of a condom. The number of sexual partners without a condom variable specifies the number of sexual partners in the last 30 days without the use of a condom. Vaginal acts without a condom are the total number of vaginal intercourse a participant had in the last 30 days without the use of a condom.

\section{Independent Variables}

The independent variables for this study included demographic variables and ethnic identity subscales. The demographic variables included were age, gender, education, country of birth, length of stay in Immokalee, and ethnicity. All the demographic information was based on participants' self-reported data. Age was calculated subtracting the date of the interview by the participant's birth date.

The Multigroup Ethnic Identity Measure (MEIM) was used to assess participants' ethnic identity level (Phinney, 1992). The MEIM is a 12-item scale that measures a participant's level of attachment with his/her ethnic group into the surrounding society. The MEIM questionnaire analyzes three aspects of ethnic identity: belonging toward participant's ethnic group, ethnic identity achievement, and ethnic behaviors and practices. The items were divided into two subscales which were used in this study: ethnic identity explore and ethnic identity belonging. Ethnic identity belonging was composed of seven items from the MEIM scale, and examined the extent to which an individual feels a sense of positive belonging to his/her ethnic group. Ethnic identity explore measured the aspect of the MEIM scale that measures the degree to which an 
individual explores or takes an active role to become better acquainted with and learn about his/her ethnic group. Ethnic identity explore consisted of five items from the MEIM scale. The MEIM score also indicated a participant's comfort level with individuals from his or her own ethnic group or from the mainstream culture of the residing area (Phinney, 1992). Dichotomous variables were created for ethnic identity belonging and ethnic identity explore at baseline and for change by splitting the continuous variable into two groups: low and high or no/negative change and positive change. The four dichotomous variables created were used to analyze any differences with demographic characteristics of the sample.

Since the two subscales measure different theoretical concepts, it is possible that they each may have different associations with risk variables. For this reason, the subscales are entered in a separate block for each multiple regression equation. The change variable for the ethnic identity scales calculated the difference between the mean score for the baseline assessment and that of the 6-month assessment. A mean score was calculated to assess the level of ethnic identity of each participant; the higher the mean MEIM score, the higher the participant's ethnic identity. The higher the ethnic identity of a participant, the higher the level of attachment and the more comfortable the participant feels with his/her ethnic group and ethnic culture. Cronbach's alpha for MEIM measure was 0.85 .

\section{Statistical Analysis}

The data were analyzed using bivariate correlations, multiple regressions, and RMANCOVA. Changes in scores for the ethnic identity subscales were included in each linear regression model that assessed changes in the risky sexual behaviors. The total 
score for MEIM was utilized for the Repeated Measures MANCOVA (RMANCOVA). All the variables were assessed to determine if they met the assumptions for linear regressions. A $\log 10$ transformation was performed on the variables in order to retain maximum power and to ensure that none of the assumptions for linear regression models were violated. The outcome variable that had a $\log 10$ transformation was the change score for vaginal acts without a condom.

The demographic variables included in the models were age, gender, education, country of birth, length of stay in Immokalee, and ethnicity. Intervention assignment was also included as a control variable in the model. The criteria for including demographic variables in the multivariate analyses depended on whether they showed a correlation approaching significance $(p<.10)$ with the dependent variables (Appendix 2).

Chi-square and independent $t$-tests were executed to assess participants' high or low ethnic identity belonging or ethnic identity explore scores at baseline with sexual risk. Similar tests were conducted to assess participants with increases in ethnic identity belonging and ethnic identity explore and participants with no change/negative changes in their ethnic identity belonging and ethnic identity explore for any differences in sexual behaviors.

Missing data were minimal in the data set among those who were followed, not exceeding a range of $0-2 \%$. A mean replacement method was used to replace missing values.

One hundred sixty-one participants from the original data set were not included in the analyses that examined changes in ethnic identity and risky sexual behaviors because they did not have 6-month follow-up data. Table 2.1 displays baseline information on those lost to follow-up, followed participants, and baseline participants. 
This study used the migrant worker sample at two time points, baseline $(n=431)$ and 6-month follow-up $(n=270)$. The post-hoc power analysis was conducted with G*Power 3 (Faul, Erdfelder, Lang, \& Buchner, 2007) for both time periods yielding power estimates ranging between .955 and .999 . This level of power provides enough strength to assume that the number of participants was sufficient for the analyses conducted in this study. The power analysis for each regression reported in this manuscript is found in Appendix 3.

All data analyses were conducted using IBM SPSS Statistics 19 (IBM Corporation). Frequencies, means, standard deviations, and chi-squares were used to examine independent variables. Multiple linear regressions were conducted to examine the relationships between changes in ethnic identity subscales and change in HIV risk behaviors. RMANCOVA was executed to examine short term change in ethnic identity total, ethnic identity belonging, and ethnic identity explore over the 6-month time period. The assignment to intervention groups was included as a covariate in the regression models executed in this study in order to reduce the likelihood of it possibly confounding the results (McCoy et al., 2009). To increase the validity of the findings, mutlicollinearity among the variables was assessed prior to the final analysis in each model presented. Employment status (whether seasonal worker or not) was excluded from the final regression model because of a high correlation with race, language, and country of birth.

\section{RESULTS}

Demographics

The mean age of the baseline sample $(n=431)$ was 41.56 years $(S D=12.39)$. Hispanics $(M=38.79, S D=11.65)$ were significantly younger than African Americans $(M=46.22, S D=12.24 ; t=6.29, p<.001)$ in the sample. The overall educational level 
was generally low in both ethnic groups. The mean level of educational achievement for the full sample was $8.45(S D=3.39)$; however, Hispanics $(M=7.30 ; \mathrm{SD}=3.25)$ were found to have a significantly lower educational level than African Americans $(M=10.39$, $S D=2.66 ; t=10.20, p<.001)$. Females accounted for $27.6 \%(n=119)$, and $30.2 \%$ of the sample were born outside of the U.S. Sixty-one percent $(n=78)$ of African Americans were single, $42 \%$ of the African Americans were female, and all the African Americans were born in the U.S. and spoke English. Hispanic females accounted for $25 \%$ of the Hispanics in the sample, and $54 \%$ of Hispanics were married.

Those participants who were lost to follow-up displayed the riskiest behaviors (highest level of crack use, alcohol use, and the highest percentage of participants with a past STI diagnosis), the lowest level of education $(M=7.37, S D=3.57 ; t=-5.108, p<$ $.001)$, and were much younger $(M=39.86, S D=11.48 ; t=-2.97, p=.027)$ than baseline and 6-month follow-up samples. Table 2.1 illustrates the demographic characteristics for baseline, 6-month follow-up, and those participants lost to follow-up. The means and standard deviations for the demographic variables and risky sexual behaviors by ethnicity at baseline and at 6-month follow-up are displayed in Appendix 4. No statistically significant differences were found between African Americans and Hispanic migrant workers in relation to risky sexual behaviors at baseline or 6-month follow-up. 
Table $2.1(5)$

Demographic Characteristics of Participants at Baseline (n=431), 6-month follow-up $(n=270$, and Lost-to-Follow Up $(n=161)$

\begin{tabular}{|c|c|c|c|c|}
\hline Measure & Baseline & $\begin{array}{l}6 \text { month Follow-up } \\
\text { or Followed }\end{array}$ & $\begin{array}{l}\text { Lost-to-Follow } \\
\text { Up or Not- } \\
\text { Followed }\end{array}$ & $p$-value \\
\hline Age $(M \pm S D)$ & $41.56 \pm 12.39$ & $42.58 \pm 12.82$ & $39.86 \pm 11.48$ & . 022 \\
\hline Education $(M \pm S D)$ & $8.45 \pm 3.39$ & $9.19 \pm 3.10$ & $7.37 \pm 3.57$ & .000 \\
\hline Female & $119(27.6 \%)$ & $90(33 \%)$ & $30(19 \%)$ & \\
\hline Male & $312(72.4 \%)$ & $180(67 \%)$ & $131(81 \%)$ & \\
\hline Marital Status $n(\%)$ & & & & .176 \\
\hline Single & $350(81.2 \%)$ & $155(57 \%)$ & $83(57 \%)$ & \\
\hline Married & $81(18.8 \%)$ & $115(43 \%)$ & $78(43 \%)$ & \\
\hline Ethnicity $n(\%)$ & & & & .001 \\
\hline Hispanic & $270(62.6 \%)$ & $142(52 \%)$ & $129(80 \%)$ & \\
\hline African American & $161(37.4 \%)$ & $128(48 \%)$ & $32(20 \%)$ & \\
\hline Prior STI Diagnosis $n(\%)$ & $102(23.7 \%)$ & $27(17 \%)$ & $75(28 \%)$ & .009 \\
\hline Number of UVA $(M \pm S D)$ & $3.26 \pm 10.15$ & $2.14 \pm 4.32$ & $4.06 \pm 17.43$ & .554 \\
\hline Number of $\mathrm{AC}(M \pm S D)$ & $103.77 \pm 138.32$ & $54.42+71.83$ & $101.09 \pm 140.44$ & .137 \\
\hline $\mathrm{MU}(M \pm S D)$ & $9.27 \pm 18.94$ & $6.60 \pm 15.91$ & $10.72 \pm 31.98$ & .122 \\
\hline $\mathrm{CU}(M \pm S D)$ & $15.61 \pm 34.24$ & $4.01 \pm 23.34$ & $27.55 \pm 85.91$ & .001 \\
\hline
\end{tabular}

Note: $\mathrm{STI}=$ Sexually Transmitted Infection; $\mathrm{SP}=$ Sexual Partners; UVA= Unprotected Vaginal Acts; $\mathrm{AC}=$ Alcohol Consumption; $\mathrm{MU}=$ Marijuana Use; $\mathrm{CU}=$ Crack Use. All Sexual acts and consumption of alcohol and illegal substances refer to the last 30 days.

Ethnic Identity and Overall Sexual Risk

At baseline $(n=431)$, males had significantly lower scores in ethnic identity explore than females $\left(\chi^{2}=3.98, p=.029\right)$. Those participants who had resided in Immokalee for more than 5 years had significantly lower scores in ethnic identity explore than participants who had relocated there more recently $\left(\chi^{2}=6.87, p=.006\right)$. Regarding ethnic identity belonging, participants who spoke English had significantly lower scores in ethnic identity belonging than Spanish speaking participants $\left(\chi^{2}=3.57, p=.038\right)$. At baseline, participants with lower scores in ethnic identity explore had a significantly higher overall sexual risk $(t=3.24, p=.001)$, a higher number of vaginal acts without a 
condom $(t=3.46, p=.001)$, and a higher consumption of alcohol in the last month $(t=$ $3.14, p=.002)$. 
Table 2.2 (6)

Differences in Ethnic Identity Explore and Ethnic Identity Belonging by Demographics and Sexual Risk ( $n=431$; 6 months: $n=270)$

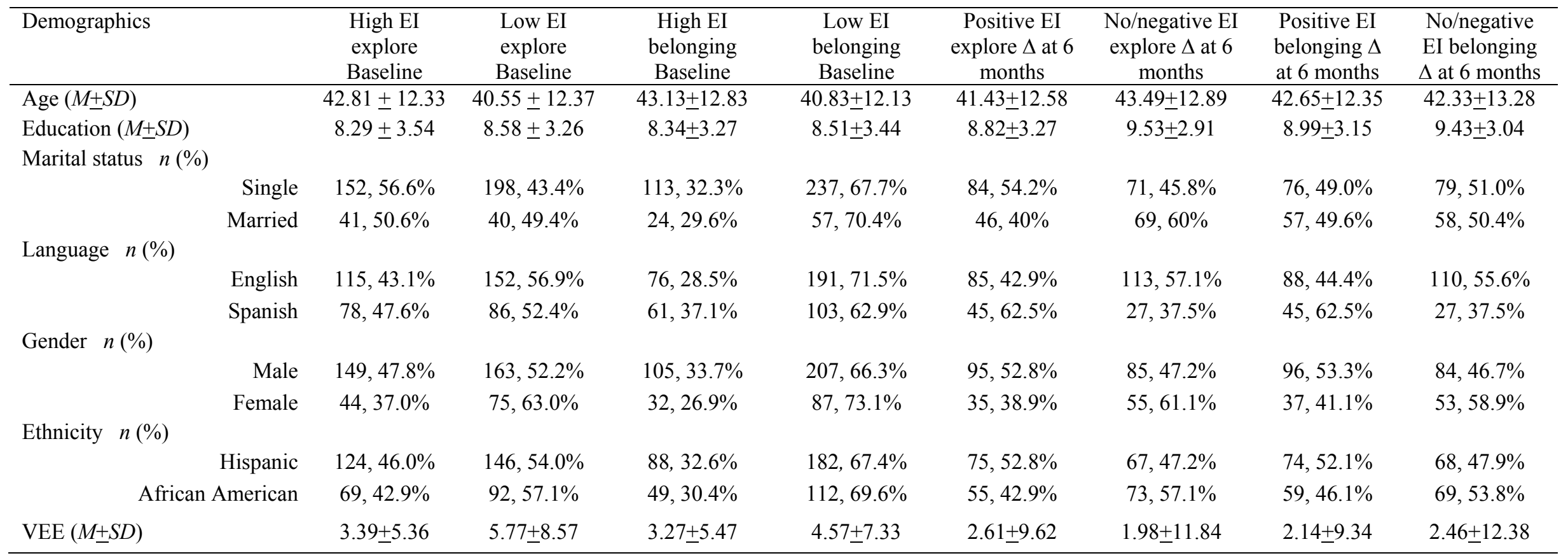

*Note: VEE=Vaginal Episode Equivalent (Sexual Risk); EI = ethnic identity. Ethnic identity explore was divided into a dichotomous dummy variable based on the mean. 


\section{HIV Risk}

The means computed for baseline $(n=431)$ risky behaviors were as follows: 3.36 vaginal sex without a condom in the last 30 days $(S D=6.967), 2.14$ sexual partners in the last 30 days $(S D=4.316)$, and .76 (approximately 1$)$ unprotected partner in the last 30 days $(S D=1.703)$. Alcoholic drinks consumed in the last month by the migrant workers in the sample were $103.77(S D=138.32)$. When comparing participants by race/ethnicity, Hispanics $(M=8.5995, S D=8.11715)$ were found to have a statistically significant higher level of sexual risk than their African American $(M=5.3552, S D=15.62187)$ counterparts as measured by the VEE index $(t=-2.107, p=.036)$. Hispanics were found to have a statistically significant higher number of sexual partners in the last 30 days than African Americans (Hispanics: $M=2.68, S D=5.086$; African Americans: $M=1.55, S D$ $=3.17 ; t=-2.175, p=.031)$ at baseline.

\section{Ethnic Identity and HIV Sexual Risk Behaviors}

Multiple linear regressions were conducted to examine the relationships between ethnic identity belonging/ethnic identity explore and HIV sexual risk variables at baseline. Then, multiple linear regressions were executed for changes in ethnic identity explore/ethnic identity belonging with changes in risky sexual behavior variables. Demographic variables and ethnic identity subscales were divided and entered into two blocks in the reported regression to assess if a relationship existed between demographic variables and risky sexual behaviors. Ethnic identity subscales were added to the model to examine how ethnic identity may influence any relationships found with risky sexual behaviors. 
Ethnic identity explore was found to be a statistically significant predictor for two of the four risky sexual behaviors assessed at baseline. The two behaviors associated with the ethnic identity subscale were VEE (a measure of overall sexual risk) and the number of vaginal acts without a condom in the last 30 days. The multiple regression for VEE revealed that the final model significantly predicted VEE, $F(8,422)=6.953, p<.001 . R^{2}$ for the model was .116 . The adjusted $R^{2}$ was .100 . Table 2.3 displays the unstandardized regression coefficients $(B)$, standard error, and standardized regression coefficients $(B)$ for ethnic identity explore and the adjusted $R^{2}$ for the model with only demographic variables and the final model with the ethnic identity explore and ethnic identity belonging. The variables in the first block contributed significantly to the prediction of level of sexual risk, $R^{2}=.067$, Adjusted $R^{2}=.053, F(6,424)=5.048, p<.001$. Statistically significant predictors of VEE in the final model were, ethnic identity explore $(\beta=-.183, t=-3.223, p<.001)$, gender $(\beta=.153, t=2.813, p=.005)$, and marital status $(\beta=.166, t=3.483, p<.001)$. Participants with a lower level of ethnic identity, male participants, and those who were married were more likely to have a higher level of sexual risk, as measured by VEE.

The multiple linear regression analyzing the number of vaginal acts without a condom was found to be statistically significant in both blocks, final block: $F(8,422)=$ $8.444, p<.001 . R^{2}$ for the model was .138 , and the adjusted $R^{2}$ was .122 . Table 2.3 displays the unstandardized regression coefficients $(B)$, standard error, and standardized regression coefficients $(B)$ for ethnic identity subscales, ethnic identity explore and ethnic identity belonging, and the demographic variables for both blocks of the regression model. Ethnic identity explore was one of the statistically significant predictors of 
vaginal acts without a condom $(\beta=-.186, t=-3.304, p=.001)$, and an inverse relationship was found between ethnic identity explore and the number of vaginal acts without a condom. Gender $(\beta=.173, t=3.232, p=.001)$ and marital status $(\beta=-.172, t=$ $3.634, p<.001$ ) were also statistically significant predictors in both blocks of the regression. Female migrant workers, married participants, and those with lower levels of ethnic identity were more likely to have a higher number of vaginal acts without a condom than their representative counterparts. Neither ethnic identity belonging nor ethnic identity explore were associated with the number of sexual partners or the number of sexual partners without a condom.

Table 2.3 (7)

Regression Analysis Summary for Ethnic Identity Predicting Risky Sexual Behaviors at Baseline $(n=431)$

\begin{tabular}{|c|c|c|c|c|c|c|c|c|c|}
\hline Outcome & Independent Variables & \multicolumn{4}{|c|}{ Block 1} & \multicolumn{4}{|c|}{ Block 2} \\
\hline & & $R^{2}$ & $B$ & $S E$ & $\beta$ & $R^{2}$ & $B$ & $S E$ & $\beta$ \\
\hline VEE & & .067 & & & & .116 & & & \\
\hline & Language & & -.124 & .093 & -.134 & & -.121 & .092 & -.130 \\
\hline & Gender & & .164 & .056 & $.162 *$ & & .154 & .055 & $.153 *$ \\
\hline & Education & & .007 & .008 & .054 & & .009 & .008 & .067 \\
\hline & Age & & -.001 & .002 & -.024 & & .000 & .002 & -.010 \\
\hline & Country of Birth & & -.068 & .095 & -.074 & & -.089 & .093 & -.096 \\
\hline & Marital Status & & .174 & .056 & $.150^{*}$ & & .192 & .055 & $.166^{*}$ \\
\hline & EI Explore & & & & & & -.189 & .059 & $-.183^{*}$ \\
\hline & EI Belonging & & & & & & -.067 & .061 & -.063 \\
\hline UVA & & .080 & & & & .138 & & & \\
\hline & Language & & -.087 & .090 & -.097 & & -.081 & .087 & -.090 \\
\hline & Gender & & .178 & .054 & $.183 *$ & & .169 & .052 & $.173^{*}$ \\
\hline & Education & & .007 & .008 & .057 & & .009 & .008 & .073 \\
\hline & Age & & -.001 & .002 & -.029 & & .000 & .002 & -.013 \\
\hline & Country of Birth & & -.021 & .091 & -.023 & & -.042 & .089 & -.047 \\
\hline & Marital Status & & .173 & .054 & $.155^{*}$ & & .191 & .053 & $.172 *$ \\
\hline & EI Explore & & & & & & -.185 & .056 & $-.186^{*}$ \\
\hline & EI Belonging & & & & & & -.086 & .058 & -.084 \\
\hline
\end{tabular}

Note: EI = ethnic identity; VEE = Vaginal Episode Equivalent (Sexual Risk); UVA = Unprotected Vaginal Acts. All Sexual acts refer to the last 30 days.

$* p \leq 0.05$ 
No statistically significant relationships were found between changes in ethnic identity belonging and/or ethnic identity explore and changes in all the four risky sexual behaviors analyzed. These regressions suggested that changes in ethnic identity belonging and ethnic identity explore had no positive or negative impact on the changes of the risky sexual behaviors.

\section{Changes in Ethnic Identity}

Regarding changes over the 6-month follow-up period in ethnic identity, participants with an increase in ethnic identity belonging and ethnic identity explore and participants with no change/negative changes in their ethnic identity belonging and ethnic identity explore were assessed for any differences in sexual behaviors. Table 2.2 describes these positive and negative/no changes in ethnic identity by demographic variables in the migrant worker sample. The table illustrates that participants with positive change in ethnic identity explore and ethnic identity belonging had a higher level of positive change in their sexual risk (VEE). This finding, however, was not found to be statistically significant.

A Repeated Measures Analysis of Covariance (RMANCOVA) was conducted to assess if any short-term changes in ethnic identity as a total measure, ethnic identity explore, and ethnic identity belonging actually took place over the 6-month follow-up period. The total measure of ethnic identity, ethnic identity explore, and ethnic identity belonging significantly changed over the 6-month assessment period (Table 2.4). After controlling for intervention assignment, language, marital status, education, gender, country of origin, and length of stay, the time effect for ethnic identity total and ethnic identity belonging was not significant $(F(1,262)=2.45, p=.119)$ and $F(1,262)=.826, p$ 
$=.364)$ respectively. However, the time effect for ethnic identity explore was found to be statistically significant after controlling for covariates, $(F(1,262)=4.02, p=.046)$, but the variance explained was very small. The changes in all three analyses were small, but were found to be statistically significant.

Table $2.4(8)$

Repeated Measures Analysis of Covariance: Mean Scores and Standard Deviations for Ethnic Identity and Subscales at Baseline $(n=431)$ and 6 Months $(n=270)$

\begin{tabular}{lccccc}
\hline & & Baseline & 6 Months & $\begin{array}{c}F \\
\text { time effect only }\end{array}$ & $\begin{array}{c}F \\
\text { covariates included }\end{array}$ \\
\hline Ethnic Identity Total & & & & $12.340^{*}$ & 2.447 \\
& $M$ & 2.8639 & 2.9784 & & \\
EI Belonging & $S D$ & .37697 & .40347 & & .826 \\
& $M$ & 3.0476 & 3.1937 & & \\
EI Explore & $S D$ & .42224 & .50141 & & $4.075^{*}$ \\
& $M$ & 2.6067 & 2.6770 & $3.752^{*}$ & \\
& $S D$ & .43125 & .45788 & & \\
\end{tabular}

Note: Covariates: language, marital status, country of birth, ethnicity, gender, intervention, length of stay.

$* p \leq 0.05$

\section{DISCUSSION}

Ethnic Identity: Risky Sexual Behaviors

Ethnic identity explore was associated with two of the four risky sexual behaviors assessed, overall sexual risk (the weighted vaginal, anal, and oral sex index) and vaginal acts without a condom in the last 30 days at baseline (refer to Table 2.3). Ethnic identity belonging was not found to have an association with overall sexual risk or vaginal acts without a condom. Ethnic identity explore was found to have an inverse relationship with vaginal acts without a condom and overall sexual risk suggesting that participants with a higher ethnic identity explore had a lower number of vaginal acts without a condom and a lower level of overall sexual risk than participants with lower ethnic identity explore scores. Both behaviors, relating to sexual acts without the use of a condom, vastly 
increase one's risk for HIV infection as well as sexually transmitted infections, reported by the CDC (Centers for Disease Control and Prevention (CDC), 2010b; Turner et al., 2009; Yi, Sandfort, \& Shidlo, 2010).

Findings from this study suggest that higher ethnic identity explore may serve as a protective factor against risky sexual behaviors, as suggested by Hernandez-Espinosa and Lefkowitz (2009) regarding the ethnic identity belonging component of the ethnic identity scale. Hernandez-Espinosa and Lefkowitz' (2009) study found that ethnic identity belonging was a protective element against risky attitudes toward sexual behaviors among African Americans, Hispanics, and European Americans (HernandezEspinosa \& Lefkowitz, 2009). Similar findings have suggested that higher levels of ethnic identity take on a protective role or a buffer effect, enhancing positive outcomes, when associated with areas like academic achievement (Kerpelman, Eryigit, \& Stephens, 2007; Schwartz, Zamboanga, \& Jarvis, 2007), higher level of quality of life (Utsey, Chae, Brown, Kelly, 2002), and a reduced level of mental stress and of positive well-being (Iwamoto \& Liu, 2010; Mossakowski, 2003; Smith et al., 1999, Utsey, Chae, Brown, \& Kelly, 2002; Yoo \& Lee, 2005).

Relating to the Identity Based Motivation theory, two of the four risky sexual behaviors analyzed could be categorized as identity-infused behaviors (These are the practices and behaviors that many ethnic/racial groups engage in to identify themselves as belonging to their ethnic group). Overall sexual risk and the number of vaginal acts without a condom are identity-infused behaviors since a link or an association does exist between them and ethnic identity. These are categorized as protective identity-infused behaviors since the higher the ethnic identity, the lower the HIV risk regarding these 
outcome variables (in relation to sexual risk, the number of vaginal sex acts without a condom, the total number of sexual partners, and the number of sexual partners without a condom).

\section{Limitations}

This study included several limitations, one of which was the brief time period assessed. Only minor changes are likely during such a short period of time. When proposing this study, I was unaware of what the changes would be over this short time period. I anticipated that these changes can be measured because of the level of stress migrant workers experience on a daily basis; however, it is likely that changes would be greater over a longer period of time. It is also encouraged that future studies be conducted on ethnic identity change and HIV risk behaviors over a longer time period, preferably over 12 months. Another limitation of this study was the bias associated with self-report. It is possible that in this high risk group of migrant workers, there may be an enhanced likelihood of inaccurate reporting, possibly among those participants with higher levels of ethnic identity. Those with higher levels of ethnic identity may have reported more protective behaviors or possibly the opposite may have occurred with the machismo stereotype found among Hispanic males. A minimal difference was found in ethnic identity from baseline to 6-month follow-up period; such a minimal difference can be a result of error. Another limitation to this study is how the 161 participants lost to followup were clearly a riskier group than the participants followed in the 6-month follow-up sample. The results regarding change may be limited to people with less risky behavior. 


\section{Future Directions}

In order to attain a clearer understanding of the mechanism of how ethnic identity may influence risky sexual behaviors, especially in this marginalized population, further studies must be implemented. This study is only a starting point for future studies, the length of time in this study was not sufficient. Future studies must include a longer time period to see how changes may affect this relationship (preferably 24 months) and emphasize examining sexual behaviors without the use of a condom.

Migrant workers are an extremely vulnerable sector of the population that tends to be overlooked due to their low socioeconomic status as well as their illegal immigration status. There have been slight improvements in the attention placed on this vulnerable group in the past decade in relation to their health, but additional work is needed to reduce their HIV risk. Future research should explore the mechanisms of how ethnic identity affects HIV risk since taking advantage of such information can assist in fine tuning prevention programs. Initiatives should be implemented that assist low-income, vulnerable groups to identify practical means to cope with ongoing challenges and to maintain a healthier lifestyle. 


\section{REFERENCES}

Albarran, C. \& Nyamathi, A. (2011). HIV and Mexican migrant workers in the United States: a review applying the vulnerable populations' conceptual model. The Journal of the Association of Nurses in AIDS Care, 22(3), 173-185. doi:10.1016/j.jana.2010.08.001

Altschul, I., Oyserman, D., \& Bybee, D. (2006). Racial-ethnic identity in midadolescence: content and change as predictors of academic achievement. Child Development, 77(5), 1155-69. doi:10.1111/j.1467-8624.2006.00926.x

Apostolopoulos, Y., Sonmez, S., Kronenfeld, J., Castillo, E., McLendon, L., \& Smith, D. (2006). STI/HIV risks for Mexican Migrant Laborers: Exploratory ethnographies. Journal of Immigrant and Minority Health, 8(3), 291-302. doi: 10.1007/s10903-0069334-2

Arcury T. A. \& Quandt, S. A. (2007). Delivery of health services to migrant and seasonal Farmworkers. Annual Review of Public Health, 28, 345-63. doi:

10.1146/annurev.publhealth.27.021405.102106

Beadnell, B., Stielstra, S., Baker, S., Morrison, D. M., Knox, K., Gutierrez, L., \& Doyle, A. (2003). Ethnic identity and sexual risk-taking among African-American women enrolled in an HIV/STD prevention intervention. Psychology, Health \& Medicine, 8(2), 187-198. doi:10.1080/1354850031000087564

Carroll, D. J, Samardick, R., Bernard, S., Gabbard S., \& Hernandez, T. (2005). Findings from the National Agricultural Workers Survey (NAWS) 2001-2001: A demographics and employment profile of United States farmworkers. (Research Report No. 9, U.S. Department of Labor).

Castro, F. G., Stein, J. A, \& Bentler, P. M. (2009). Ethnic pride, traditional family values, and acculturation in early cigarette and alcohol use among Latino adolescents. The Journal of Primary Prevention, 30(3-4), 265-92. doi:10.1007/s10935-009-0174-z

Cislo, A. M. (2008). Ethnic Identity and self-esteem: Contrasting Cuban and Nicaraguan young adults. Hispanic Journal of Behavioral Sciences, 30(2), 230-250. doi:10.1177/0739986308315297

Cole, B., Matheson, K., \& Anisman, H. (2007). The moderating role of ethnic identity and social support on relations between well-being and academic performance. Journal of Applied Social Psychology, 37(3), 592-615. doi:10.1111/j.1559-1816.2007.00176.x

Cooper, M. L. (2002). Alcohol use and risky sexual behavior among college students and youth: evaluating the evidence. Journal of Studies on Alcohol. Supplement, (14), 101-17. Retrieved from http://www.ncbi.nlm.nih.gov/pubmed/12022716 
Coppel, J., Dumont, J., \& Visco, I. (2001). Trends in immigration and economic consequences. OECD Economics Department Working Papers, No. 284, OECD Publishing. Retrieved from http://dx.doi.org/10.1787/553515678780

Denner, J., Organista, K. C., Dupree, J. D., \& Thrush, G. (2005). Predictors of HIV transmission among migrant and marginally housed Latinos. AIDS and Behavior, 9(2), 201-210.

Dermen, K. H., \& Cooper, M. L. (1994). Sex-related alcohol expectancies among adolescents: I. Scale development. Psychology of Addictive Behaviors, 8(3), 152-160. doi:10.1037/0893-164X.8.3.152

Duke, M. R. \& Carpinteiro, F. J. G. (2009). The effects of problem drinking and sexual risk among Mexican migrant workers on their community of origin. Human Organization, 68(3), 328-344. doi:10.1016/j.bbi.2008.05.010

Faul, F., Erdfelder, E., Lang, A.-G., \& Buchner, A. (2007). G*Power 3: A flexible statistical power analysis for the social, behavioral, and biomedical sciences. Behavior Research Methods, 3(2)9, 175-191. doi: 10.3758/BF03193146

Fernandez, M. I., Collazo, J. B., Hernandez, N., Bowen, G. S., Varga, L. M., Vila, K. C., Arheart, K. L., \& Perrino, T. (2004). Predictors of HIV risk among Hispanic farm workers in South Florida: Women are at higher risk than men. AIDS and Behavior, 8(2), 165-173. doi:10.1023/B:AIBE.0000030247.00140.62.

Gwyther, E. (1998). Migrant Farmworker Children: Health Status, barriers to care, and nursing innovations in health care delivery. Journal of Pediatric Health Care, 12(2), 6066. doi:10.1016/S0891-5245(98)90223-1

Hansen, E \& Donohoe, M. (2003). Health issues of migrant and seasonal farmworkers. Journal of Health Care For The Poor And Underserved, 14(2), 153-164. doi:10.1177/1049208903251513

Hiott, A. E., Gryzywacz, J. G., Davis, S. W., Quandt, S. A., \& Arcury, T. A. (2008). Migrant farmworker stress: mental health implications. The Journal of Rural Health, 24(1), 32-39. doi:10.1111/j.1748-0361.2008.00134.x

Hirsch, S., Higgins, J., Bentley, M. E., \& Nathanson, C. A. (2002). The social constructions of sexuality: marital infidelity and sexually transmitted disease-HIV risk in a Mexican migrant community. American Journal of Public Health, 92(8), 1227-1237. doi: 10.2105/AJPH.92.8.1227

Johnson, A. M., Mercer, C. H., Erens, B., Copas, A. J., McManus, S., Wellings, K., . . Field, J. (2001). Sexual behavior in Britain: Partnerships, practices, and HIV risk behaviors. Lancet, 358(9296), 1835-42. doi:10.1016/S0140-6736(01)06883-0 
Kalichman, S. C., Simbayi, L., Jooste, S., Vermaak, R., \& Cain, D. (2008). Sensation seeking and alcohol use predict HIV transmission risks: prospective study of sexually transmitted infection clinic patients, Cape Town, South Africa. Addictive Behaviors, 33(12), 1630-3. doi:10.1016/j.addbeh.2008.07.020

Kiang, L., Witkow, M. R., Baldelomar, O. A., \& Fuligni, A. J. (2010). Change in ethnic identity across the high school years among adolescents with Latin American, Asian, and European backgrounds. Journal of Youth and Adolescence, 39(6), 683-93. doi:10.1007/s10964-009-9429-5

Kiene, S. M., Barta, W. D., Tennen, H., \& Armeli, S. (2009). Alcohol, helping young adults have unprotected sex with casual partners: Findings from a daily diary study of alcohol use and sexual behavior. Journal of Adolescent Health, 44 (1), 73-80.

Kissinger, P., Liddon, N., Schmidt, N., Curtin, E., Salinas, O., \& Narvaez, A. (2008). $\mathrm{HIV/STI}$ risk behaviors among Latino migrant workers in New Orleans post-hurricane Katrina disaster. Sexually Transmitted Diseases, 35(11), 924-9.

doi:10.1097/OLQ.0b013e31817fa2cc

Leigh, B. C. (1990). The relationship of sex-related alcohol expectancies to alcohol consumption and sexual behavior. British Journal of Addiction, 85(7), 919-28.

doi: 10.1111/j.1360-0443.1990.tb03722.x

Marcia, J. E. (1966). Development and validation of ego identity status. Journal of Personality and Social Psychology, 3(5), 551-558.

Marsiglia, F. F., Kulis, S., Hecht, M. L., \& Sills, S. (2004). Ethnicity and ethnic identity as predictors of drug norms and drug use among preadolescents in the US Southwest. Substance Use \& Misuse, 39(7), 1061-1094. doi:10.1081/JA-120038030

Martínez-Donate, A. P., Rangel, M. G., Hovell, M. F., Santibáñez, J., Sipan, C. L., Izazola, J.A. (2005). HIV infection in mobile populations: the case of Mexican migrants to the United States. Revista Panamerican Salud Publica, 17(1), 26-9.

McCabe, S. E., Morales, M., Cranford, J. A., Delva, J., McPherson, M. D., \& Boyd, C. J. (2007). Race/ethnicity and gender differences in drug use and abuse among college students. Journal of Ethnicity in Substance Abuse, 6(2), 75-95.

doi:10.1300/J233v06n02_06

McCoy, H. V., Hlaing, W. M., Ergon-Rowe, E., Samuels, D., \& Malow, R. (2009). Lessons from the fields: A migrant HIV prevention project. Public health Reports, 124(6), 790-6. Retrieved from http://www.ncbi.nlm.nih.gov/pmc/articles/PMC2773941/

Muñoz-Laboy, M., Hirsch, J. S., \& Quispe-Lazaro, A. (2009). Loneliness as a sexual risk factor for male Mexican migrant workers. American Journal of Public Health, 99(5), 802-10. doi:10.2105/AJPH.2007.122283 
Oesterle, S., Hawkins, J. D., \& Hill, K. G. (2011). Men's and women's pathways to adulthood and associated substance misuse. Journal of Studies on Alcohol and Drugs, 72(5), 763-773. Retrieved from http://www.ncbi.nlm.nih.gov/pubmed/21906504

Organista P.B., Organista K.C., \& Soloff, P.R. (1998). Exploring AIDS related knowledge, attitudes, and behaviors of female migrant workers. Health and Social Work. 23(2):96-103.

Organista, K. C., Kubo, A. (2005). Pilot survey of HIV risk and contextual problems and issues in Mexican/Latino migrant day laborers. Journal of Immigrant Health, 7(4), 269281.

Organista, K. C. \& Organista, P. B. (1997). Migrant laborers and AIDS in the United States: Review of the literature. AIDS Education and Prevention, 9, 83-93.

Organista, K. C., Carrillo, H., \& Ayala, G. (2004). HIV prevention with Mexican migrants: Review, critique, and recommendations. Journal of Acquired Immune Deficiency Syndromes, 37, S227-39.

Organista, K. C., Organista, P. B., Bola, J. R., Garcia de Alba J. E., \& Moran, M. A. C. (2000). Predictors of condom use in Mexican migrant laborers. American Journal of Community Psychology, 28:245-265.

Parker, R. G., Easton, D., \& Klein, C. H. (2000, June). Structural barriers and facilitators in HIV prevention: a review of international research. AIDS (London, England).

Retrieved from http://www.ncbi.nlm.nih.gov/pubmed/10981471

Persky, I., \& Birman, D. (2005). Ethnic identity in acculturation research: A study of multiple identities of Jewish refugees from the former Soviet Union. Journal of CrossCultural Psychology, 36, 557-572. doi: 10.1177/0022022105278542

Phinney, J. S. (1992). The Multigroup Ethnic Identity Measure: A new scale for use with diverse groups. Journal of Adolescent Research, 7(2), 156-176.

doi:10.1177/074355489272003

Phinney, J. S., Horenczyk, G., Liebkind, K., \& Vedder, P. (2001). Ethnic identity, immigration, and well-being: An interactional perspective. Journal of Social Issues, 57, 493-510.

Roberts, R. E., Phinney, J. S., Masse, L. C., Chen, Y. R., Roberts, C. R., \& Romero, A. (1999). The structure of ethnic identity of young adolescents from diverse ethnocultural groups. The Journal of Early Adolescence, 19(3), 301-322.

doi:10.1177/0272431699019003001

Rhodes, S. D., Bischoff, Ã. W. E., Burnell, J. M., Whalley, L. E., Walkup, M. P., Vallejos, Q. M., Quandt, S. A., et al. (2010). HIV and sexually transmitted disease risk 
among male Hispanic / Latino migrant farmworkers in the Southeast: Findings from a pilot CBPR Study. American Journal of Industrial Medicine, (December 2009). doi:10.1002/ajim.20807

IBM Corporation. (2010). IBM SPSS Base 19.0 for Windows User's Guide. IBM Corporation, New York.

Susser, E., Desvarieux, M., \& Wittkowski, K. M. (1998). Reporting sexual risk behavior for HIV: a practical risk index and a method for improving risk indices. American Journal of Public Health, 88(4), 671-4. doi: 10.2105/AJPH.88.4.671

Torres, V., Martinez, S., Wallace, L. D., Medrano, C. I., Robledo, A. L., \& Hernandez, E. (2012). The connection between Latino ethnic identity and adult experiences. Adult Education Quarterly, 62(1), 3-18. doi:10.1177/0741713610392765

Utsey, S., Chae, M. H., Brown, C. F., \& Kelly, D. (2002). Effect of ethnic Group membership on ethnic identity, race-related stress, and quality of life. Cultural Diversity and Ethnic Minority Psychology, 8(4), 366-377. doi:10.1037/1099-9809.8.4.367

Villarejo, D., McCurdy, S. A., Bade, B., Samuels, S., Lighthall, D., \& Williams III, D. W. (2010). The health of California's immigrant hired farmworkers. American Journal of Industrial Medicine, 53(4), 387-397. doi:10.1002/ajim.20796

Weatherby, N. L., McCoy, H. V., Bletzer, K. V., McCoy, C. B., Inciardi, J. A., McBride, D. C., \& Forney, M. A. (1997). Immigration and HIV among migrant workers in rural Southern Florida. Journal of Drug Issues, 27(1), 155-173. Retrieved from http://www.mendeley.com/research/immigration-hiv-among-migrant-workers-ruralsouthern-florida/

Winkleby, M. A., Snider, J., Davis, B., Jennings, M. G., \& Ahn, D. K. (2003). Cancerrelated health behaviors and screening practices among Latinos: Findings from a community and agricultural labor camp survey. Ethnicity and Disease, 13(3), 376-386. [PubMed: 12894963]

Worby, P. A., \& Organista, K. C. (2007). Alcohol Use and Problem Drinking Among Male Mexican and Central American immigrant laborers: A Review of the Literature. Hispanic Journal of Behavioral Sciences, 29(4), 413-455. doi:10.1177/0739986307308765

Zamboanga, B. L. (2005). Alcohol expectancies and drinking behaviors in Mexican American college students. Addictive Behaviors, 30(4), 673-84.

doi:10.1016/j.addbeh.2004.08 


\section{Chapter VI}

\section{TRANSITION TO MANUSCRIPT 3: MODERATOR ANALYSIS}

In Manuscript 3, multiple linear regressions were conducted to assess if Social Proficiency Scale (SPS; social support) change contributes to the prediction of changes in ethnic identity. An analysis of social support change was conducted to examine any possible moderator effects with ethnic identity in contributing to the prediction of HIV risk behaviors. A moderator, as defined by Frazier, Tix, and Baron (2004), is a "variable that alters the direction or strength of the relation between a predictor and an outcome" (p. 116). The method utilized to assess moderator effects was guided by following two articles: one by Baron and Kenny (1986) and the previously mentioned article by Frazier, Tix, and Baron (2004). Mediation effects were not assessed because ethnic identity would have to have a direct relationship with social support. Examination of the relationships between these variables did not yield significant results.

The moderator effect analysis was briefly addressed in Manuscript 3 . Since none of the models were significant, however, they were not described in detail in the publishable manuscript. The results not reported in the manuscript are found in Appendix 8 of this dissertation. The moderator effect analyses conducted for ethnic identity as a total measure are displayed in Tables 8.1 through 8.11 (found in Appendix 8). Tables 8.12 through 8.22 describe the moderator effect analysis conducted for ethnic identity belonging, and Tables 8.23 through 8.32 displays the results of the same analyses conducted for ethnic identity explore. Multiple linear regression analyses were conducted to examine if changes in social support was a moderator for the relationship between 
ethnic identity and HIV risk behavior variables. Significant results were not found in these analyses.

The linear regressions were composed of four blocks for each model analyzed. A $z$-score was computed for social support change, since this was the moderator proposed in the hypotheses. The first block included only demographic variables. Ethnic identity change was included in the second block of the regression, and the $z$ - score for social support change was added in the third block of the model. The fourth block included all previously added variables with the addition of the computed interaction variables (the product of $z$-score of social support change and ethnic identity change). In order for a moderator relationship to be declared, the model must be significant in the last two blocks of the regression. The same procedure was used for the MEIM subscales, ethnic identity belonging and ethnic identity explore. The interaction variables must also be significant (Frazier, Tix, and Baron, 2004).

Moderator effects of social support change on ethnic identity change in relation to the dependent variables specified in the proposed hypotheses for research question 4 were examined through multiple linear regressions. These dependent variables included: Vaginal Episode Equivalent (VEE) change, number of partners change, number of sexual partners without a condom change, vaginal acts without a condom change, alcohol consumption change, marijuana usage change, crack usage change, Sex Related Alcohol Expectancy (SRAE) total change, SRAE-enhanced sex change, SRAE-decreased nervousness change, and SRAE-increased risk change. The independent variables for the models presented in this study included ethnic identity total change and demographic 
data. No significant models were found to support moderator effect hypotheses of social support change with HIV risk behaviors.

The analyses that supported the hypotheses proposed and yielded significant models are described in detail in the following manuscript. Significant models were found suggesting positive ethnic identity change was associated with positive changes in social support. Ethnic identity did change over the 6-month assessment period. However, intervention assignment was not a covariate that contributed to ethnic identity change. 


\title{
MANUSCRIPT 3
}

\section{THE ROLE OF SOCIAL SUPPORT WITH ETHNIC IDENTITY AMONG SEXUALLY ACTIVE MIGRANT WORKERS}

\begin{abstract}
Background: Migrant workers are one of the most rapidly growing populations in the United States (U.S.) and have been significantly affected by HIV/AIDS. This longitudinal study examines changes in ethnic identity, changes in social support, and their combined impact on HIV risk behaviors among migrant workers in a rural community in Florida. Methods: Baseline and 6-month assessment self-reported data were collected from a larger HIV intervention study among migrant workers in Immokalee, Florida $(n=270)$. The Multigroup Ethnic Identity Measure (MEIM) was used to measure ethnic identity. Repeated Measures MANCOVA was utilized to assess differences in ethnic identity over the 6-month time period relating to intervention group assignment. Linear regressions were conducted to examine the nature of the relationship between short term changes in ethnic identity and potentially protective factors such as social support. Changes in social support were examined as a moderator for changes in ethnic identity and HIV risk behaviors (sexual behaviors and substance use).
\end{abstract}

Results: Increases in social support were significantly associated with increases in ethnic identity. Social support did not moderate the relationship between changes in ethnic identity and HIV risk behaviors. The participants' intervention assignments had no bearing on changes in ethnic identity and/or ethnic identity subscales.

Conclusion: The positive relationship between changes in social support and changes in ethnic identity suggest that efforts to address ethnic identity may result by attempting to 
increase social support networks for migrant workers. Social support should be further explored to better understand its potentially positive role in ethnically diverse populations.

Key Words: ethnic identity, HIV risk, migrant workers, social support 


\section{BACKGROUND}

The South Florida region has been severely affected by the HIV epidemic, with elevated risks being detected in the migrant worker community (Fernandez et al., 2009). Migrant workers are disproportionately affected by HIV due to poverty, social isolation (Muñoz-Laboy, Hirsch, \& Quispe-Lazaro, 2009), lack of access to health care and lack of knowledge (Rhodes et al., 2010; Villajero, 2003). The seroprevalence rate of HIV among migrant workers is estimated to be between $2.5 \%$ and $13 \%$, almost 10 times the national rate (Hansen \& Donahoe, 2003). Research has suggested that migrant workers are at a heightened level of risk for HIV due to their engagement in a number of HIV risk behaviors, such as risky sexual behaviors and alcohol and substance use (Duke \& Carpintiero, 2009; Fernandez et al., 2004; Weatherby et al., 1997).

The place of origin for the majority of migrant workers is Mexico. Hispanics from Mexico and South and Central America account for about 70 percent of the migrant worker population (Villajero, 2010). Migrant workers are a highly transient community making it difficult to properly monitor the incidence and prevalence rates of HIV (Hansen \& Donohoe, 2003). Low education and low income status are contributors in making migrant workers highly susceptible to negative health outcomes and HIV infection (Martinez-Donate et al., 2005; McCoy, Hlaing, Ergon-Rowe, Samuels, \& Malow, 2009).

Lack of access to and availability of health care services, social isolation, language barriers, and constant mobility are some of the challenges migrant workers face because of migration. Underreporting of health conditions among migrant workers is common because of the limited access to health care services, limited availability of 
health care services, and the fear of loss of wages. Underreporting of medical conditions contribute to the lack of data on illnesses throughout the migrant worker community (Hansen \& Donohoe, 2003). Social isolation has been a result of language barriers that migrants experience in their new country of residence. One coping mechanism used to deal with many of the hardships in their way of life is engaging in sexual risk behaviors and substance and alcohol abuse (Hirsch Higgins, Bentley, \& Nathanson, 2002).

\section{Ethnic Identity}

Ethnic identity, as defined by Phinney, DuPont, Espinosa, Revil, and Sanders (1994), is “a feeling of belonging to one's group, a clear understanding of the meaning of one's membership, positive attitudes toward the group, familiarity with its history and culture and involvement in its practices" (p.169). Ethnic identity includes aspects of ethnic involvement such as language, behavior, values, and knowledge of ethnic group history (Persky \& Birman, 2005; Salgado de Snyder, Díaz Pérez, \& Maldonado, 2006). Phinney further defines the components of ethnic identity as: ethnic identity explore and ethnic identity belonging (Phinney, 1992). Ethnic identity "belonging," includes ethnic pride, positive feelings toward one's ethnic group, and satisfaction with being a member of one's ethnic group. Ethnic identity "explore" measures the extent to which individuals search for a sense of meaning in their ethnicity (Phinney, 1992). A moderating factor to changes in the development and exploration of ethnic identity in individuals is being a member of an ethnic minority group. In addition to being a minority, developmental levels of ethnic identity tend to be stronger in females than in males (Kiang, Witkow, Baldelomar, \& Fuligni, 2009). 
Acculturation is a concept that is commonly confused with the term ethnic identity. Ethnic identity, which is the construct assessed in this study, measures the level of attachment one has with his/her ethnic group while acculturation measures how one from an ethnic/minority group adapts to the mainstream culture. Acculturation and ethnic identification have been used as one construct in the past, even though it is crucial to recognize their differences in order to clearly understand how they may impact behaviors. Acculturation is associated with changes in attitudes or beliefs in relation to the mainstream or dominant culture. This concept is usually measured at a group level, while ethnic identification is assessed at the individual level. Ethnic identification measures the individual's emotional attachment (comfort level) and the engagement in cultural practices of his/her ethnic group (Phinney, 1992). This study focuses on level of ethnic identity reported by the migrant worker sample.

In terms of health, studies have suggested that ethnic identity may be associated with positive health behaviors, such as healthy lifestyle practices, which may consist of minimal alcohol consumption, no substance use, and employing HIV prevention practices (Brook, Whiteman, Balka, Win, \& Gursen, 1998; Nesdale, Rooney, \& Smith, 1997). Although the literature on the relationship between ethnic identity and risky sexual behaviors is sparse, several studies have been conducted among a number of ethnic groups. It must be noted that the studies that report on ethnic identity have not assessed ethnic identity change and how it may impact individuals' health behaviors. Some of the studies present a theory of ethnic identity as a protection against engagement in risky sexual and substance use behaviors. For example, in a study conducted by Beadnell and colleagues (2003), a "buffering" effect was shown among African American heterosexual 
females. There was a difference in risky sexual acts in the last four months depending on their level of ethnic identity: those with higher levels of ethnic identity had lower levels of risky sexual behaviors compared to the women with lower ethnic identity levels (Beadnell, et al., 2003). Decline in identifying with one's ethnic group, found among those who have been in the U.S. for longer periods of time, has been linked to higher levels of depressive symptoms as well as lower self-esteem as found in a study by Umana-Taylor and Updegraff (2007) on Latino adolescents.

The literature suggests that ethnic identity changes over time, depending on the stage of development of an individual and life changing circumstances (Phinney, 1992, 2003). A study administered by Kiang, Witkow, Baldelomar, and Fuligni (2010) focusing on ethnic identity change in high school students over a four year time period revealed that increases in ethnic identity were associated with the level of closeness these high school students felt toward their parents and their same ethnic peer relationships. Increases in ethnic identity were found in students whose ethnic group was not a majority in the school. Unfortunately, this study did not assess if such changes coincided with increases in positive outcomes such as academic achievement or any HIV risk behaviors (Kiang, Witkow, Baldemar, \& Fuligni, 2010).

Social support has been consistently linked with lower levels of mortality and morbidity (Cole, Matheson, \& Anismen, 2007; Lee and Rotheram-Borus, 2001; Lyyra \& Heikkinen, 2006; Rutledge et al., 2004). These findings may be a result of the effect of social support, including the health promoting element - social networks may influence an individual's health behaviors and practices, such as cigarette smoking and alcohol consumption (Uchino, 2006). In the literature, social support has been repeatedly 
associated with more positive health outcomes, such as higher infant birth weight (Campos et al., 2008; Dunkel-Schetter, Gurung, Lobel, \& Wadhwa, 2000). It has also been suggested that social support provides a buffer against stressful conditions which indirectly affect health outcomes (Dunkel-Schetter, Gurung, Lobel, \& Wadhwa, 2000; Sagrestano, Feldman, Killingsworth-Rini, Woo, \& Dunkel-Schetter, 1999).

\section{Social Support and Ethnic Identity}

Studies have shown that higher levels of ethnic identity were associated with stronger social support networks. Individuals with higher ethnic identity also had a higher likelihood of utilizing social support services (Lopez, Antonio, Fekete, Penedo, 2010; Yoo \& Lee, 2005). The component of ethnic identity that addresses the closeness that individuals feel with their group may open doors to access social support and other services from members of their ethnic group. Both factors may contribute to more positive outcomes in relation to health as well as academic achievement (Noh \& Kaspar, 2003; Phinney, Horenczyk, Liebkind, \& Vedder, 2000). A study by Cole and her colleagues (2007) with adolescent ethnic minorities suggested that ethnic identity and social support were moderating variables to the mental wellbeing of participants as well as academic performance. Another study by Lopez and her colleagues (2010) concluded that women living with HIV who acquired high levels of ethnic identity had less perceived stress, and this was suggested to be a result of better coping and more social support resources (Lopez, Antoni, Fekete, \& Penedo, 2010). These studies describe how social support and ethnic identity seem to provide a protective shield against negative health outcomes and academic failure. 
The focus of this study is to examine the impact that changes in social support have on changes in ethnic identity and ultimately, HIV risk behaviors among migrant workers in South Florida. If such a relationship exists, do changes in social support moderate the relationship between changes in ethnic identity and changes in risky sexual and substance use behaviors? The relationships being tested in this study are based on the premise that increases in social support will have a direct relationship with increases in ethnic identity and may be predictors of positive changes in HIV risk behaviors. The sexual behaviors analyzed for the moderator effect includes the number of sexual partners, the level of sexual risk, the number of unprotected (without a condom) sexual partners, and the number of unprotected (without a condom) vaginal acts. In addition, this study explores the impact of social support on ethnic identity.

\section{METHODS}

\section{Sample}

Data were collected from a larger study assessing the effectiveness of an enhanced/adapted cognitive behavioral intervention, Peer Education Ends Risky Behaviors (PEER), compared to a health promotion program, Health Education Always Leads To a Healthy You (HEALTHY), for producing long-term reductions in HIV risk and increased health behaviors among alcohol and other drug using migrant workers (McCoy, Hlaing, Ergon-Rowe, Samuels, \& Malow, 2009). Both interventions were conducted in English and Spanish. Participants chose the intervention language with which they felt most comfortable (McCoy et al., 2009).

The intervention group assignment was used as a covariate in the regression models executed in this study (McCoy et al., 2009) in order to control its effects on 
changes of the constructs being explored. The interesting component that differentiates these two interventions, which may impact ethnic identity, is how the PEER intervention included telenovelas (Hispanic soap operas) and movies that depicted ethnically specific scenarios regarding HIV risk, for African American and Hispanic cultures. These ethnically specific components were not found in the health promotion program (HEALTHY). Although, this difference may affect changes in ethnic identity, neither intervention was designed to change ethnic identity. However, because of the potential impact on ethnic identity, the intervention assignment was controlled for in the regressions conducted in this study.

Participants were migrant workers residing in Immokalee, an agricultural area in Collier County, Florida. Participants were recruited using a targeted sampling method. Inclusion criteria were the following: age 18 years or older; at least one episode of unprotected (without a condom) vaginal, anal, and/or oral sex; alcohol or other drug intake in the past 90 days; and fluency in English and/or Spanish. After the sample was recruited, participants were randomly assigned into one of the two intervention groups, HEALTHY or PEER (McCoy et al., 2009).

The present study was limited to participants who were present for both baseline and 6-month follow-up assessment $(n=270)$. The study defined potential participants as migrant workers who conformed to the Public Health Services Act (PHSA; 1944) definition; however, the inclusion criteria was broadened to include people who work or worked indirectly in agriculture, such as in packing houses.

The theory that guided this study is known as the Identity Based Motivation Theory (Oyserman, Yoder, \& Fryberg, 2007). This theory focuses on how ethnic/racial 
groups perceive accepted and unaccepted behaviors based on the perspective of a member of their ethnic/racial group known as identity-infused behaviors. This theory best described the link that exists between ethnic identity and high risk behaviors, which will be helpful for the moderator effect analysis being executed. In many cases identityinfused behaviors are protective.

\section{Measures}

\section{Dependent Variables}

The dependent variables were self-reported and collected through the HIV/AIDS Risk Reduction Questionnaire (HRRQ), composed of 204 questions and covered 13 sections, utilized in the parent study. There were three ethnic identity variables used: ethnic identity total, ethnic identity belonging, and ethnic identity explore. The Multigroup Ethnic Identity Measure (MEIM) was used to assess participants' ethnic identity level (Phinney 1992, Roberts et al., 1999). The MEIM was 12-items that measure a participant's level of attachment to his/her ethnic group. The questionnaire is divided into two components that were analyzed in this study: ethnic identity explore and ethnic identity belonging. Ethnic identity explore measured the extent to which an individual searches for a sense of meaning in his/her ethnicity. Ethnic identity belonging included feelings toward ethnic pride, positive feelings toward one's ethnic group and one's satisfaction with being a member of his/her ethnic group. Ethnic identity subscales were included in each linear regression model as well as ethnic identity as a total measure. Ethnic identity subscales were included in the models to better understand the type of ethnic identity that may be contributing to the prediction of the level of risk. The scores for each item utilize a Likert scale, ranging from 1 (strongly disagree) to 4 (strongly 
agree). A mean score was calculated to assess the level of ethnic identity of each participant; the higher the mean MEIM score, the higher the participant's ethnic identity. Cronbach's alpha for the instrument was 0.85 . The subscales for ethnic identity from the MEIM questionnaire were computed and included in each regression model. Ethnic identity belonging (Cronbach's $\alpha=0.85$ ) examined the extent to which individuals feel positive belonging to their ethnic group. Ethnic identity belonging was composed of 7 items from the MEIM scale. Ethnic identity explore (Cronbach's $\alpha=0.51)$ measured the degree to which individuals explore or take an active role to become better acquainted with and learn about their ethnic group. Ethnic identity explore was created with 5 items from the MEIM scale.

\section{HIV Risk Behaviors}

The dependent variables were self-reported and collected through the HIV/AIDS Risk Reduction Questionnaire (HRRQ) utilized in the parent study. Seven dependent variables, labeled as HIV risk behaviors and alcohol expectancies, were examined and included in the moderator regression models. Change variables were computed for each HIV risk behavior listed below by subtracting the frequency of each risk behavior at baseline from that at the 6-month assessment period. The change variable for the ethnic identity scales calculated the difference in the mean score for the baseline assessment and the 6-month assessment. The HIV risk behavior variables were vaginal equivalent episode (VEE; defined below), number of partners, number of sexual partners without a condom, number of vaginal acts without a condom, the amount of alcohol consumed in the past 30 days, the number of times marijuana was used in the past 30 days, and the number of times crack was used in the past 30 days. 
VEE weights oral, vaginal, and anal sex acts based on their differential risk.

Compared to using individual specific sex acts (e.g., unprotected vaginal intercourse) as individual variables, this index has the advantage of providing a score reflecting subjects' overall sexual risk-taking behavior (Susser, Desvarieux, and Wittkowski, 1998). Higher VEE scores represent higher sexual risk. The formula for computing VEE is: (number of unprotected vaginal sex acts $)+(2 \mathrm{x}$ number of unprotected anal sex acts $)+(0.01 \mathrm{x}$ number of unprotected oral sex acts) (Susser et al., 1998).

The number of sexual partners' variable sums the number of sexual partners in the last 30 days. The number of unprotected sexual partners variable specifies the number of partners with whom the participant engaged in sex without a condom in the last 30 days. Vaginal acts without a condom are the sum of the number of vaginal sexual encounters without a condom for the participant in the last 30 days. Alcohol consumption was measured by the number of drinks in the last 30 days. Marijuana and crack usage included the number of times the participant used marijuana and/or crack in the last 30 days.

\section{Independent Variables}

The inclusion criteria for the demographic variables in the regression models depended on whether they showed a correlation approaching significance $(p<.10)$ with the dependent variable. The demographic variables included in the models were age, gender, education, length of stay in Immokalee, and ethnicity.

The Social Provisions Scale (SPS) examined the degree to which respondents' social relationships provide various dimensions of social support. SPS will be referred to as social support throughout this manuscript. This 24 -item scale was a multi-dimensional 
construct. The responses utilize a Likert scale and range from 1 (strongly agree) to 5 (strongly disagree) (Leigh, 1990, p. 922). The Cronbach's alpha for SPS was 0.80.

\section{Statistical Analysis}

Repeated Measures Analysis of Covariance (RMANCOVA) was used to assess changes in ethnic identity across time while controlling for group design (PEER v. HEALTHY) and the interaction between ethnic identity change and intervention assignment group. To increase the validity of the findings, mutlicollinearity among the variables was examined prior to determining the predictors to be included in each model presented. Employment status (whether seasonal worker or not) was excluded from the RMANCOVA and final regression model because of high correlation with language ( $r=$ $.291, p=.001)$ and country of birth $(r=.291, p<.000)$. Analyses were run for the full sample $(n=270)$.

Demographics such as ethnicity, marital status, gender, education, migration status, and the intervention variable were included in the models as covariates. The demographic variables that were found to approach significance $(p<.10)$ regarding their Pearson $r$ coefficients were included in the linear regressions conducted in this study. By limiting the number of predictor variables to significant correlations, the number of variables included in each model was reduced. There was no evidence of mutlicollinearity among the predictor variables. Intervention group assignment was included in the regression models to control for the effects of the intervention.

Dichotomous variables were created for changes in ethnic identity belonging and changes in ethnic identity explore in order to conduct independent $t$-tests and chi square analyses with demographic variables and changes in social support. 
Moderator effect analyses were conducted to explore if changes in social support acts as a moderator between changes in ethnic identity and changes in HIV risk behaviors. The moderator effect, as explained in an article by Frazier, Tix, and Baron (2004), "alters or strengthens" an already existing relationship between the predictor and the outcome variable. $Z$-scores were calculated for the changes in ethnic identity and ethnic identity subscales, and social support, following Frazier, Tix, and Barron's (2004) technique for analyzing moderator effects. Interaction effects were created for the $z$ scores of ethnic identity and ethnic identity change subscales with the $z$-scores for changes in social support. The linear regression models included four blocks. The first block consisted of demographic variables such as age, marital status, length of stay, gender, ethnicity and education. Ethnic identity change was added in the second block. The third block included social support change. The interaction variables were included in the fourth block.

In addition to the analyses executed to assess the moderator effect for social support, multiple linear regressions were executed to assess the relationship between ethnic identity and the ethnic identity subscales with social support. Two block multiple linear regressions were executed to better understand the relationship between demographics and social support with ethnic identity and ethnic identity subscales, ethnic identity belonging and ethnic identity explore. The first block of the regression included only demographic variables, and then a change in social support was added in the second block. All the variables were assessed to determine if they met the assumptions for linear regressions. VEE, number of vaginal acts without a condom, number of sexual partners, alcohol use, crack use, and marijuana use were the outcome variables that did not meet 
the assumptions of linear regression due to high kurtosis levels. Log 10 transformations were conducted on the variables mentioned in order to retain maximum power and to ensure that none of the assumptions for linear regression models were violated. It is of great importance to be aware of how "robust" linear regression analysis is, and if one or more assumptions are not met, it has been reported that unbiased and efficient results will still be achieved (Edgell \& Noon, 1984; Havlick \& Peterson, 1977; Hayes, 1996). The issue arises when there is a combination of a sample size less than 120 and there is extreme non-normality in the variables analyzed. These two requirements for nonnormality were not present in the migrant worker data set used in this analysis (Edgell \& Noon, 1984; Havlick \& Peterson, 1977; Hayes, 1996).

Change scores were computed for ethnic identity belonging, ethnic identity explore, social support, and the HIV risk behaviors included in the moderator analyses. These change variables were computed by calculating the difference between the selfreported data for 6-month-follow-up and baseline data.

Missing

Missing data was minimal in the data set utilized for this study, not exceeding a range of $0-2 \%$. A mean replacement method was used to rectify this issue.

\section{Lost to Follow-Up}

One hundred sixty-one participants from the original data set were not included in the analyses since they did not have 6-month follow-up data. This represents about 37\% of the participants assessed at baseline. Those lost to follow-up included 32 African Americans (67\% male) and 129 Hispanics (84\% male). Approximately $81 \%$ of the African Americans were single compared to $43 \%$ of the Hispanic participants. The mean 
education level for these participants was 7.37 grade $(S D=3.57)$ and the average age was 39.86 years $(S D=11.48)$. The mean number of risky sexual behaviors among this group was as follows: 3.84 vaginal acts in the last 30 days $(S D=7.74)$ and 4.06 sexual partners in the last 30 days $(S D=17.43)$. These participants had a mean of 101.09 drinks in the last 30 days $(S D=140.44)$. With regard to substance use, $60 \%(n=96, M=10.72$ times in the last 30 days, $S D=31.98$ ) consumed marijuana in the last 30 days, while $34 \%$ $(n=55, M=27.55$ times in the last 30 days, $S D=85.91)$ consumed crack in the last 30 days. Differences between those who were followed and those who were lost to followup were analyzed and are discussed in the results section.

Sample Size and Power Analysis

For the purpose of this study, the sample size was 270 participants. The software utilized for the power analyses was G*Power 3 (Faul, Erdfelder, Lang, \& Buchner, 2007). The power analysis conducted assessed medium effect size of .50 with an $\alpha$ of .05 and power of .95 resulting in a suggested sample size of 178 . This requirement was met and statistical analyses were conducted on the sample. All data analyses were conducted using IBM SPSS Statistics 19 (IBM Corporation). The power for each outcome variable is found in Appendix 3 (Table 3.2).

\section{RESULTS}

\section{Demographics}

The number of participants at baseline was 431 . There were 270 study participants with both baseline and 6-month data who were included in these analyses. The sample is composed of $67 \%(n=180)$ males and $33 \%$ females; the average achieved educational level was $9^{\text {th }}$ grade $(M=9.19, S D=3.10)$. The mean age of the total sample 
was 42.5 years $(S D=12.76)$. Thirty-three percent of the full sample was female $(n=90)$, and $57 \%(n=180)$ of the full sample was single. The sample was composed of both Hispanic $(n=142,52 \%)$ and African American $(n=128,48 \%)$ ethnicities. A majority of the participants were Hispanic $(n=106,75.0 \%)$ males, and $54 \%$ of them were single. The means computed for baseline risky behaviors were as follows: 3.36 vaginal acts without a condom in the last 30 days $(S D=6.97)$ and 2.14 sexual partners in the last 30 days $(S D=4.32)$. Approximately $54(S D=71.83)$ alcoholic drinks were consumed in the last 30 days by the migrant workers in the sample. The participants consumed crack approximately four times in the last 30 days $(n=57 ; S D=23.34)$, while marijuana was consumed approximately seven times in the last 30 days $(n=109 ; S D=15.91)$.

When comparing cases not included in the analyses and those followed, there were a number of significant differences between the groups (See Table 3.1 below). The lost cases were significantly younger $(M=39.86)$ than the cases that participated $(M=$ 42.58) in the 6-month assessment period $(t=-2.172, p=.027)$, and lost cases had a significantly lower level of education $(M=7.37)$ compared to participants $(M=9.19)$ included in this study $(t=-5.207, p<.001)$. A large portion of the lost cases were of Hispanic ethnicity $\left(80 \%, p<.001, \chi^{2}=31.99\right)$, and lost cases consumed significantly larger amounts of crack in the last 30 days compared to the followed cases $(M=27.55 \mathrm{v}$. $M=4.01$, respectively; $t=4.209, p<.001)$. 
Table $3.1(9)$

Baseline, 6-month follow-up, and Lost-to-Follow Up data sets by Demographic

Characteristics

\begin{tabular}{|c|c|c|c|c|}
\hline Measure & Baseline $(n=431)$ & $\begin{array}{l}\text { 6-month Follow-up } \\
\text { or } \\
\text { Followed }(n=270)\end{array}$ & $\begin{array}{l}\text { Lost-to-Follow } \\
\text { Up or Not- } \\
\text { Followed }(n=161)\end{array}$ & $p$-value \\
\hline Age $(M \pm S D)$ & $41.56+12.39$ & $42.58+12.82$ & $39.86 \pm 11.48$ & .022 \\
\hline $\begin{array}{l}\text { Education }(M \pm S D) \\
\text { Gender } n(\%)\end{array}$ & $8.45+3.39$ & $9.19 \pm 3.10$ & $7.37 \pm 3.57$ & $\begin{array}{l}.000 \\
.001\end{array}$ \\
\hline Female & $119(27.6 \%)$ & $90(33 \%)$ & $30(19 \%)$ & \\
\hline Male & $312(72.4 \%)$ & $180(67 \%)$ & $131(81 \%)$ & \\
\hline Marital Status $n(\%)$ & & & & .176 \\
\hline Single & $350(81.2 \%)$ & $155(57 \%)$ & $83(57 \%)$ & \\
\hline Married & $81(18.8 \%)$ & $115(43 \%)$ & $78(43 \%)$ & \\
\hline Ethnicity $n(\%)$ & & & & .001 \\
\hline Hispanic & $270(62.6 \%)$ & $142(52 \%)$ & $129(80 \%)$ & \\
\hline African American & $161(37.4 \%)$ & $128(48 \%)$ & $32(20 \%)$ & \\
\hline Prior STI Diagnosis $(n(\%)$ & $102(23.7 \%)$ & $27(17 \%)$ & $75(28 \%)$ & .009 \\
\hline Number of UVA $(M \pm S D)$ & $3.26 \pm 10.15$ & $2.14 \pm 4.32$ & $4.06 \pm 17.43$ & .554 \\
\hline Number of $\mathrm{AC}(M \pm S D)$ & $103.77 \pm 138.32$ & $54.42 \pm 71.83$ & $101.09 \pm 140.44$ & .137 \\
\hline $\mathrm{MU}(M \pm S D)$ & $9.27 \pm 18.94$ & $6.60 \pm 15.91$ & $10.72 \pm 31.98$ & .122 \\
\hline $\mathrm{CU}(M \pm S D)$ & $15.61 \pm 34.24$ & $4.01 \pm 23.34$ & $27.55 \pm 85.91$ & .001 \\
\hline
\end{tabular}

Note: $\mathrm{STI}=$ Sexually Transmitted Infection; $\mathrm{SP}=$ Sexual Partners; UVA= Unprotected Vaginal Acts; $\mathrm{AC}=$ Alcohol Consumption; $\mathrm{MU}=$ Marijuana Use; $\mathrm{CU}=$ Crack Use. All Sexual acts and consumption of alcohol and illegal substances refer to the last 30 days.

Ethnic identity belonging and ethnic identity explore were investigated between those with an increase in ethnic identity belonging or ethnic identity explore scores and no change/negative change in ethnic identity belonging or ethnic identity explore scores by demographic variables. Table 3.2 , found below, describes these positive and negative/no change in ethnic identity by demographic variables in the migrant worker sample. The table also illustrates that those with positive change in ethnic identity explore and ethnic identity belonging had had a greater increase in social support within the 6month assessment period than those participants who showed negative/no change in the ethnic identity subscales (ethnic identity belonging: $t=-5.198, p=.001$; ethnic identity explore: $t=-3.220, p=.001)$ (Table 3.2). 
Table $3.2(10)$

Differences in Ethnic Identity Explore change and Ethnic Identity Belonging Change by Demographics and Social Support $(n=270)$

\begin{tabular}{|c|c|c|c|c|}
\hline Demographics & $\begin{array}{l}\text { Positive EI } \\
\text { explore } \Delta\end{array}$ & $\begin{array}{l}\text { No/negative EI } \\
\text { explore } \Delta\end{array}$ & $\begin{array}{l}\text { Positive EI } \\
\text { belonging } \Delta\end{array}$ & $\begin{array}{c}\text { No/negative EI } \\
\text { belonging } \Delta\end{array}$ \\
\hline Age $(M+S D)$ & $41.43+12.58$ & $43.49 \pm 12.89$ & $42.65+12.35$ & $42.33+13.28$ \\
\hline Education $(M+S D)$ & $8.82+3.27$ & $9.53+2.91$ & $8.99+3.15$ & $9.43+3.04$ \\
\hline \multicolumn{5}{|l|}{ Marital status $n(\%)$} \\
\hline Single & $84,54.2 \%$ & $71,45.8 \%$ & $76,49.0 \%$ & $79,51.0 \%$ \\
\hline Married & $46,40 \%$ & $69,60 \%$ & $57,49.6 \%$ & $58,50.4 \%$ \\
\hline \multicolumn{5}{|l|}{ Language $n(\%)$} \\
\hline English & $85,42.9 \%$ & $113,57.1 \%$ & $88,44.4 \%$ & $110,55.6 \%$ \\
\hline Spanish & $45,62.5 \%$ & $27,37.5 \%$ & $45,62.5 \%$ & $27,37.5 \%$ \\
\hline \multicolumn{5}{|l|}{ Gender $n(\%)$} \\
\hline Male & $95,52.8 \%$ & $85,47.2 \%$ & $96,53.3 \%$ & $84,46.7 \%$ \\
\hline Female & $35,38.9 \%$ & $55,61.1 \%$ & $37,41.1 \%$ & $53,58.9 \%$ \\
\hline \multicolumn{5}{|l|}{ Ethnicity $n(\%)$} \\
\hline Hispanic & $75,52.8 \%$ & $67,47.2 \%$ & $74,52.1 \%$ & $68,47.9 \%$ \\
\hline African American & $55,42.9 \%$ & $73,57.1 \%$ & $59,46.1 \%$ & $69,53.8 \%$ \\
\hline Social Support (SPS) $(M+S D)$ & $7.74+13.44$ & $2.41+13.76$ & $9.77 \pm 14.66$ & $0.31+11.22$ \\
\hline
\end{tabular}

*Note: EI: Ethnic Identity. Ethnic identity explore and Ethnic identity belonging were divided into two categories based on the mean and created into a dichotomous variable.

\section{Ethnic Identity Total and Ethnic Identity Subscales with Social Support}

Multiple linear regressions were performed to assess the nature of the relationship between ethnic identity total change, ethnic identity belonging, and ethnic identity explore with changes in social support. The first model examines the nature of the relationship between ethnic identity total change and changes in social support. The standardized regression $(\beta)$, the standard error $(S E)$, and the unstandardized regression coefficients $(B)$ for changes in social support are displayed in Table 3.3. The first block included the demographic variables. Correlations between changes in ethnic identity total, ethnic identity explore, and ethnic identity belonging and demographic variables are found in Appendix 7 mentioned previously. Social support was added in the second block. Social support total change was found to be a significant predictor of ethnic identity total change. $R^{2}$ for the final block was .215 , and the adjusted $R^{2}$ was .194 . The 
final block of the regression model was significant, $F(7,262)=10.237, p<.001$. The variables in the first block did not contribute significantly to the prediction of the magnitude of ethnic identity total change $\left(R^{2}=.037\right.$, Adjusted $R^{2}=.015, F(6,263)=$ $1.690, p=.124)$. The examination of standardized regression coefficients of the second block demonstrated that the only significant predictor was social support change ( $\beta=$ $.431, t=7.699, p<.001)$. Increases in social support from baseline to 6 months were associated with increases in the level of ethnic identity over the 6-month period.

The next regression assessed if changes in social support were associated with changes in ethnic identity belonging. The first block was composed of the demographic variables mentioned earlier and the second block included the change in social support total. The standardized regression $(\beta)$, the standard error $(S E)$, and the unstandardized regression coefficients $(B)$ for the predictors of the two block regression model are displayed in Table 3.3 for changes in social support. At the end of the final step, including all predictor variables, $R$, representing proportion of variability, was significantly different from zero $\left(R^{2}=.166\right.$, Adjusted $R^{2}=.144, F(7,262)=7.467, p<$ .001). The variables in the first block did not significantly contribute to the prediction of the measure of ethnic identity belonging change $\left(R^{2}=.044\right.$, Adjusted $R^{2}=.022, F(6,263)$ $=2.023, p=.063)$. Social support change in the final model was the only significant predictor of ethnic identity change $(\beta=.358, t=6.197, p<.001)$. All coefficients were in the predicted direction, thus suggesting that increases in social support change are significantly associated with an increase in the magnitude of ethnic identity belonging change. 
The last regression assessed the relationship between ethnic identity explore change and social support change. The first block included the demographic variables mentioned previously, and social support was added in the second block. Social support change was found to have a statistically significant relationship with ethnic identity explore change. The standardized regression $(\beta)$, the standard error $(S E)$, and the unstandardized regression coefficients $(B)$ for changes in social support are displayed in Table 3.3. $R^{2}$ for the model was .109 , and adjusted $R^{2}$ was .085 . The final block of the model was significant, $F(7,262)=4.588, p<.001$. The variables in the first block did not contribute significantly to the prediction of the measure of ethnic identity explore change $\left(R^{2}=.047\right.$, Adjusted $\left.R^{2}=.025, F(6,263)=2.168, p=.046\right)$. The inclusion of social support total change in the final block contributed to prediction of ethnic identity explore change $(\beta=.255, t=4.273, p<.001)$. Marital status contributed to the prediction of ethnic identity explore change in both blocks; Block $1:(\beta=-.180, t=-2.940, p=.004)$ and Block 2: $(\beta=-.177, t=-2.996, p=.003)$. The results suggest that single participants and those who increased their scores in social support change were more likely to increase their ethnic identity explore score over the 6-month period. 
Table 3.3 (11)

Regression Analysis Summary for Social Support Predicting Ethnic Identity ( $n=270)$

\begin{tabular}{|c|c|c|c|c|c|c|c|c|c|}
\hline Outcome & Independent & & $\mathrm{Blc}$ & & & & & $\mathrm{ck} 2$ & \\
\hline \multirow{9}{*}{$\mathrm{EI} \Delta$} & & $R^{2}$ & $B$ & $S E$ & $\beta$ & $R^{2}$ & $B$ & $S E$ & $\beta$ \\
\hline & & .037 & & & & .215 & & & \\
\hline & Gender & & -.103 & .077 & -.090 & & -.049 & .070 & -.043 \\
\hline & Education & & -.008 & .013 & -.048 & & -.006 & .012 & -.035 \\
\hline & Country of Birth & & .070 & .102 & .057 & & .024 & .092 & .019 \\
\hline & Length of Stay & & -.068 & .086 & -.051 & & -.038 & .078 & -.029 \\
\hline & Marital Status & & -.100 & .067 & -.091 & & -.095 & .061 & -.087 \\
\hline & Intervention & & .027 & .066 & .025 & & .004 & .060 & .004 \\
\hline & Social Support $\Delta$ & & & & & & .017 & .002 & $.431 *$ \\
\hline \multirow[t]{8}{*}{ EI belonging $\Delta$} & & .040 & & & & .166 & & & \\
\hline & Gender & & -.091 & .097 & -.063 & & -.034 & .091 & -.024 \\
\hline & Education & & -.008 & .016 & -.035 & & -.005 & .015 & -.025 \\
\hline & Country of Birth & & .154 & .128 & .100 & & .106 & .120 & .069 \\
\hline & Length of Stay & & -.034 & .109 & -.020 & & -.003 & .102 & -.002 \\
\hline & Marital Status & & -.105 & .084 & -.076 & & -.101 & .079 & -.073 \\
\hline & Intervention & & .138 & .083 & .101 & & .114 & .078 & .084 \\
\hline & Social Support $\Delta$ & & & & & & .018 & .003 & $.358 *$ \\
\hline \multirow[t]{8}{*}{ EI explore $\Delta$} & & .047 & & & & .109 & & & \\
\hline & Gender & & -.082 & .085 & -.064 & & -.047 & .083 & -.037 \\
\hline & Education & & -.003 & .014 & -.018 & & -.002 & .014 & -.010 \\
\hline & Country of Birth & & -.001 & .113 & -.001 & & -.031 & .110 & -.023 \\
\hline & Length of Stay & & -.151 & .096 & -.102 & & -.131 & .093 & -.088 \\
\hline & Marital Status & & -.219 & .074 & -.180 & & -.216 & .072 & -.177 \\
\hline & Intervention & & .006 & .073 & .005 & & -.009 & .071 & -.008 \\
\hline & Social Support $\Delta$ & & & & & & .011 & .003 & $.255^{*}$ \\
\hline
\end{tabular}

Note: Note: EI= Ethnic identity; Adj. = adjusted.

$* p \leq 0.05$

Moderator Effect Analysis

Multiple linear regressions were conducted to assess moderator effects of social support with ethnic identity total and ethnic identity subscales, ethnic identity belonging and ethnic identity explore. $Z$-scores were computed to allow for analysis for moderator effects (Kenny \& Baron 1986; Frazier, Tix, and Barron, 2004). These analyses did not reveal any statistically significant models which led to the conclusion that changes in social support did not moderate the relationship between changes in ethnic identity and HIV risk behaviors and alcohol expectancies. 


\section{Intervention Assignment Group Analysis}

A Repeated Measures Analysis of Covariance (RMANCOVA) was conducted to assess the magnitude of change for ethnic identity total between the two intervention groups, PEER and HEALTHY, at the two time points (baseline and 6-month follow-up) while controlling for language, marital status, education, gender, country of origin, and immigration status. The RMANCOVA analysis for the full ethnic identity measure revealed a non-significant result for the group by time effect, $F(1,262)=.208, p=.649$. A significant time effect, irrespective of intervention status, was found with ethnic identity increasing between baseline and 6 months, $F(1,262)=12.54, p<.001$. The groups, irrespective of time, did not differ, $F(1,262)=1.34, p=.248$. Similar results were yielded for ethnic identity belonging and ethnic identity explore (ethnic identity belonging: $F(1,262)=.367, p=.545$; ethnic identity explore: $F(1,262)=.005, p=$ .942). The groups, irrespective of time, did not differ for ethnic identity total, ethnic identity belonging, and ethnic identity explore, as shown in Table 3.4. 
Table 3.4 (12)

Repeated Measures Analysis of Covariance for Ethnic Identity Levels by Assignment of Intervention Group (PEER \& HEALTHY) $(n=270)$

\begin{tabular}{|c|c|c|c|c|c|c|c|c|}
\hline $\begin{array}{l}\text { Ethnic } \\
\text { Identity }\end{array}$ & $\begin{array}{c}\text { PEER } \\
\text { Baseline } \\
(n=148\end{array}$ & $\begin{array}{c}\text { PEER } \\
6 \text { Months } \\
(n=148)\end{array}$ & $\begin{array}{c}\text { HEALT } \\
\text { HY } \\
\text { Baseline } \\
(n=122) \\
\end{array}$ & $\begin{array}{c}\text { HEALTHY } \\
6 \text { Months } \\
(n=122)\end{array}$ & $\begin{array}{c}\text { Total } \\
\text { Baseline } \\
(n=270)\end{array}$ & $\begin{array}{c}\text { Total } \\
6 \\
\text { Months } \\
(n=270) \\
\end{array}$ & $\begin{array}{c}F \\
\text { Time } \\
\text { Effect } \\
\text { Only } \\
\end{array}$ & $\begin{array}{c}F \\
\text { Covariates } \\
\text { Included }\end{array}$ \\
\hline $\begin{array}{r}\text { EI total** } \\
M \\
S D\end{array}$ & $\begin{array}{l}2.8525 \\
.35861\end{array}$ & $\begin{array}{l}2.9482 \\
.42630\end{array}$ & $\begin{array}{l}2.8777 \\
.39918\end{array}$ & $\begin{array}{l}3.0150 \\
.37237\end{array}$ & $\begin{array}{l}2.8639 \\
.37697\end{array}$ & $\begin{array}{l}2.9784 \\
.40347\end{array}$ & $12.535^{*}$ & .208 \\
\hline $\begin{array}{l}\text { EI } \\
\text { Belonging }\end{array}$ & & & & & & & $14.385^{*}$ & 2.389 \\
\hline $\begin{array}{r}M \\
S D\end{array}$ & $\begin{array}{l}3.0290 \\
.40849\end{array}$ & $\begin{array}{l}3.1477 \\
.52546\end{array}$ & $\begin{array}{l}3.0703 \\
.43897\end{array}$ & $\begin{array}{l}3.2494 \\
.46665\end{array}$ & $\begin{array}{l}3.0476 \\
.42224\end{array}$ & $\begin{array}{l}3.1937 \\
.50141\end{array}$ & & \\
\hline $\begin{array}{r}\text { EI Explore } \\
M \\
S D\end{array}$ & $\begin{array}{l}2.6054 \\
.40200\end{array}$ & $\begin{array}{l}2.6689 \\
.48020\end{array}$ & $\begin{array}{l}2.6082 \\
.46596\end{array}$ & $\begin{array}{l}2.6869 \\
.43102 \\
\end{array}$ & $\begin{array}{l}2.6067 \\
.43125\end{array}$ & $\begin{array}{l}2.6770 \\
.45788\end{array}$ & $3.744 *$ & .005 \\
\hline
\end{tabular}

\section{DISCUSSION}

This study makes a number of contributions to the present literature on migrant workers and the impact of social support on their engagement in HIV risk behaviors. Social support was found to be significantly associated with changes in ethnic identity and ethnic identity subscales. Social support was a significant predictor of ethnic identity and of ethnic identity subscales, ethnic identity belonging and ethnic identity explore.

Previous literature found that social support was a mediator of ethnic identity and adjustment to college for Latino college students (Schneider \& Ward, 2003). High levels of ethnic identity have also been associated with stronger social support as well as a higher likelihood of accessing social support services (Lopez, Antonio, Fekete, \& Penedo, 2010). The literature suggests that ethnic identity has a direct relationship with social support. Evaluations of the effects of social support generally assessed a single point in time rather than changes over time. In this study changes in social support were 
found to be associated with changes in ethnic identity for the migrant workers in this sample.

Previous studies have generally assessed changes in social support in relation to health outcomes and psychological well-being. These studies found social support to be a buffer to negative health outcomes (Clay, Roth, Wadley, \& Haley, 2008; GaylordHarden, Ragsdale, Mandara, Richards, \& Peterson, 2007; Lyyra \& Heikinen, 2006). For example, a study analyzing HIV positive Latinas' positive and negative coping styles concluded that those individuals that engaged in positive coping styles, such as obtaining social support, were found to consume fewer cigarettes and less alcohol, and showed greater adherence to their HIV medication (Sanchez, Rice, Stein, Milburn, \& RotheramBorus, 2010). The previously mentioned studies suggest that social support may play a vital role in influencing individuals' health status as well as the types of health behaviors they practice.

Social support has also been found to be a protective factor against risky sexual behaviors, in previous research. A previous study suggested that low social support placed young females at higher risk of engaging in risky sexual behaviors which put them at risk for STIs, especially HIV (Mazaferro et al., 2006). It has also been found that social support is associated directly with substance abuse and indirectly with risky sexual behaviors, illustrating that social support takes on a protective characteristic indirectly with risky sexual behaviors (Peterson, Buser, \& Westberg, 2010). The same indirect relationship may be present in our study since social support and ethnic identity are directly associated with each other and ethnic identity has been demonstrated to associated with HIV risk behaviors (Shehadeh, McCoy, Rubens, Batra, Renfrew, \& 
Winter, 2011). Social support may ultimately be indirectly associated with HIV risk behaviors in the same fashion.

The changing environment in which one lives may be another contributor to ethnic identity explore. A recent study by Phinney (2008) explains that ethnic identity exploration in emerging adults is affected by the environment in which they find themselves. Phinney posits that if one resides in a constantly changing environment, this may influence one's level of ethnic identity explore. This phenomenon seems to be applicable to the migrant worker sample examined in this study since their ethnic identity did change over time. These changes may be a result of how they live under a constant stress of not having a stable employment status and housing arrangements.

The primary predictor of ethnic identity explore change was marital status. Single participants were more likely to increase their level of ethnic identity explore than their married counterparts. This finding suggests that single individuals, since they may not be as settled as their married counterparts, may need to seek out more information and more contact with their ethnic group as a means of coping with their environment.

Utilization of social support is a manner of coping with the new environment for immigrants and assists them to adapt to their new way of life. Particularly among minority groups, strong social support has been associated with higher levels of ethnic identity. In many cases, strong levels of group identification may improve group support or allow such support to be accessible (Matheson \& Anisman, 2007; Phinney, Horenczyk, Liebkind, \& Vedder, 2001). Ultimately, such resources assist immigrants in adapting to their environment in a more efficacious manner. 
There were small but statistically significant short term changes in ethnic identity and ethnic identity subscales for the migrant workers in the sample over the 6-month time period assessed. However, it was concluded that the reported changes were related to a time effect only. The magnitude of change did not differ by group assignment. There is no literature that examines changes in ethnic identity in relation to assignment to intervention groups similar to the analyses conducted in this study. However, there have been interventions that have been based on ethnic matching, such as the one conducted by Field and Caetano (2010), which was based on a brief alcohol intervention. Field and Caetano (2010) concluded that being ethnically matched resulted in an enhancement in the effectiveness of the intervention on the participants. In this study, the Hispanic participants who were a part of the Spanish-language groups might have experienced similar enhancement.

\section{Limitations}

The limitations of the study must be noted. One limitation is that the data reported were self-reported, therefore potentially increasing bias and may not be as accurate as other forms of reporting. Another limitation is that there was a minimal difference found between ethnic identity from baseline and 6-month follow-up period. This minimal difference can be a result of error. Finally, the time period over which the data was analyzed was relatively short. It is possible that a longer follow-up period would have led to different findings.

\section{Implications}

Future studies on ethnic identity among migrant workers may be helpful in reducing HIV risk. It is too early to state that it would be beneficial to include ethnic 
identity indicators in future interventions. However, it is important to further understand the link between ethnic identity and social support and how such a relationship may be beneficial in lowering HIV risk among migrant workers. Future studies should be conducted over a longer period of time to better assess this relationship and possible factors to reduce HIV risk behaviors. It has been reported that culturally sensitive HIV/STI prevention interventions are beneficial for ethnic/minority groups. Future studies can also examine culturally sensitive indicators more closely. It has also been reported that including concepts that are familiar in contexts in which the different groups reside can be beneficial. Including ethnically appropriate context was implemented in the HIV risk reduction intervention used in the parent study. Since the intervention targeted cultural sensitivity factors, it may have influenced the increases in ethnic identity that were observed in our study. The low wages, difficult employment situations, and other stressors in the environment of migrant workers, make them highly vulnerable to engaging in unhealthy and risky behaviors. Migrant workers need more attention in the area of research in order to better their quality of health and reduce the risks found in this marginalized community. 


\section{REFERENCES}

Baron, R. M. \& Kenny, D. A. (1986). The moderator-mediator variable distinction in social psychological research: Conceptual, strategic, and statistical considerations. Journal of Personality and Social Psychology, 51(6), 1173-1182.

McLellan-Lemal, E., O’Daniels, C. M., Marks, G., Villar-Loubet, O., Doherty, I. A., Simpson, C., . . . Borkowf, C. B. (2011). Sexual risk behaviors among AfricanAmerican and Hispanic women in five counties in the Southeastern United States: 20082009. Women's Health Issues, 22(1), e9-e18. doi:10.1016/j.whi.2011.06.002

Brook, J. \& Pahl, K. (2005). The protective role of ethnic and racial identity and aspects of an Africentric orientation against Drug Use among African American young adults. Journal of Genetics, 166(3), 329-345. Retrieved from http://www.ncbi.nlm.nih.gov/pmc/articles/PMC1315285/

Brown, E. J., \& Van Hook, M. (2006). Risk behavior, perceptions of HIV risk, and risk reduction behavior among a small group of rural African American women who use drugs. Journal of the Association of Nurses in AIDS Care, 17(5), 42-50.

Campos, B., Schetter, C. D., Abdou, C. M., Hobel, C. J., Glynn, L. M., \& Sandman, C. A. (2008). Familialism, social support, and stress: Positive implications for pregnant Latinas. Culture Diversity Ethnic Minority Psychology, 14(2), 155-162. doi: 10.1037/1099-9809.14.2.155

CDC. (2004). HIV/AIDS among Hispanics. Centers for Disease Control and Prevention, National Center for HIV, STD, and TB Prevention, Division of HIV/AIDS Prevention, October.

Centers for Disease Control and Prevention. (2009). HIV/AIDS in the United States. Retrieved from http://www.cdc.gov/hiv/resources/factsheets/us.htm.

Cislo, A. M. (2008). Ethnic identity and self-esteem contrasting Cuban and Nicaraguan young adults. Hispanic Journal of Behavioral Sciences, 30(2), 230-250. doi: $10.1177 / 0739986308315297$

Clay, O. J., Roth, D. L., Wadley, V. G., \& Haley, W. E. (2008). Changes in social support and their impact on psychosocial outcome over a 5-year period for African American and White dementia caregivers. Geriatric Psychiatry, 23(8), 857-862. doi: 10.1002/gps.1996

Cole, B., Matheson, K., \& Anisman, H. (2007). The moderating role of ethnic identity and social support on relations between well-being and academic performance. Journal of Applied Social Psychology, 37(3), 592-615. doi:10.1111/j.1559-1816.2007.00176.x 
Cooper, M. L. (2002). Alcohol use and risky sexual behavior among college students and youth: evaluating the evidence. Journal of Studies on Alcohol. Supplement, 14, 10117. Retrieved from http://www.ncbi.nlm.nih.gov/pubmed/12022716

Cutrona, C. E., \& Russell, D. W. (1987). The Provisions of social relationships and adaptation to stress. Advances in Personal Relationships, 1, 37-67.

Denner, J., Organista, K. C., Dupree, J. D., \& Thrush, G. (2005). Predictors of HIV Transmission among Migrant and marginally housed Latinos. AIDS and Behavior, 9(2), 201-210. doi: 10.1007/s10461-005-3901-3

Dermen, K. H., \& Cooper, M. L. (1994). Sex-related alcohol expectancies among adolescents: I. Scale development. Psychology of Addictive Behaviors, 8(3), 152-160. doi:10.1037/0893-164X.8.3.152

Duke, M. R. \& Carpinteiro, F. J. G. (2009). The effects of problem drinking and sexual risk among Mexican migrant workers on their Community of Origin. Human Organization, 68(3), 328-344. doi:10.1016/j.bbi.2008.05.010

Edgell, S. E. \& Noon, S. M. (1984). Effect of violation of normality on the $t$-test of the correlation coefficient. Psychological Bulletin, 95(3), 576-583.

Espinosa-Hernandez, G. \& Lefkowitz, E. S. (2009). Sexual behaviors and attitudes and ethnic identity during college. Journal of Sex Research, 46(5), 471-482.

doi:10.1080/00224490902829616

Farmer, M. A. \& Meston, C. M. (2006). Predictors of condom use self-efficacy in an ethnically diverse university sample. Archives of Sexual Behavior, 35(3), 313-326. doi: $10.1007 / \mathrm{s} 10508-006-9027-5$

Faryna, E.L., \& Morales, E. (2000). Self-efficacy and HIV-related risk behaviors among multiethnic adolescents. Cultural Diversity and Ethnic Minority, 6(1), 42-56.

doi:10.1037/1099-9809.6.1.42

Faul, F., Erdfelder, E., Lang, A.-G., \& Buchner, A. (2007). G*Power 3: A flexible statistical power analysis for the social, behavioral, and biomedical sciences. Behavior Research Methods, 3(2)9, 175-191. doi: 10.3758/BF03193146

Fernandez, M. I., Collazo, J. B., Hernandez, N., Bowen, G. S., Varga, L. M., Vila, K. C., Arheart, K. L., \& Perrino, T. (2004). Predictors of HIV risk among Hispanic farm workers in South Florida: Women are at higher risk than men. AIDS and Behavior, 8(2), 165-173. doi:10.1023/B:AIBE.0000030247.00140.62

Field, C. \& Caetano, R. (2010). The role of ethnic matching between patient and provider on the effectiveness of brief alcohol interventions with Hispanics. Alcoholism: Clinical and Experimental Research, 34(2), 262-271. doi:10.1111/j.15300277.2009.01089.x 
Frazier, P. A., Tix, A. P., \& Barron, K. E. (2004). Testing moderator and mediator effects in counseling psychology research. Journal of Counseling Psychology, 51(1), 115-134.

Golub, S.A., Walker, J. J., Longmire-Avital, B., Bimbi, D. S., \& Parsons, J. T. (2010). The role of religiosity, social support, and stress-related growth in protecting against HIV risk among transgender women. Journal of Health Psychology, 15(8), 1135-44. doi: $10.1177 / 1359105310364169$

Gwyther, E. (1998). Migrant farmworker children: Health status, barriers to care, and nursing innovations in health care delivery. Journal of Pediatric Health Care, 12(2), 6066. doi:10.1016/S0891-5245(98)90223-1

Hansen, E \& Donohoe, M. (2003). Health issues of migrant and seasonal farmworkers. Journal of Health Care For The Poor And Underserved, 14(2), 153-164. doi:10.1177/1049208903251513

Havlick, L.L. \& Peterson, N. L. (1977). Effect of the violation of assumptions upon significance levels of the Pearson r. Psychological Bulletin, 84(2), 373-377.

Hayes, A. F. (1996). Permutation test is not distribution free: Testing $\mathrm{H}_{0}: p=0$. Psychological Methods, 1(2), 184-198.

Hiott, A. E., Gryzywacz, J. G., Davis, S. W., Quandt, S. A., \& Arcury, T. A. (2008). Migrant farmworker stress: mental health implications. The Journal of Rural Health, 24(1), 32-39. doi:10.1111/j.1748-0361.2008.00134.x

Hirsch, S., Higgins, J., Bentley, M. E., \& Nathanson, C. A. (2002). The social constructions of sexuality: marital infidelity and sexually transmitted disease-HIV risk in a Mexican migrant community. American Journal of Public Health, 92(8), 1227-1237. doi: 10.2105/AJPH.92.8.1227

Hood, K. B. (2009). The Impact of Ethnic Identity and Group Support on HIV Intervention Outcomes (master's thesis). Virginia Commonwealth University, Richmond, Virginia.

Hussey, J. M., Hallfors, D. D., Waller, M. W., Iritani, B. J., Halpern, C. T., Bauer, D. J. (2007). Sexual behavior and drug use among Asian and Latino adolescents: Association with immigrant status. Journal of Immigrant \& Minority Health, 9(2), 85-94.

Iturbide, M. I., Rafaelli, M., \& Carlo, G. (2009). Protective effects of ethnic identity on Mexican American college students' psychological well-being. Hispanic Journal of Behavioral Sciences, 31(4), 536-552.

Iwamoto, D. K., \& Liu, W. M. (2010). The Impact of racial identity, ethnic identity, Asian values and race-related stress on Asian Americans and Asian international college students' psychological well-being. Journal of Counseling Psychology, 57(1), 79-91. 
Kang, S. Y., Deren, S., Andia, J., Colon, H. M., \& Robles, R. (2004). Effects of changes of perceived self-efficacy on HIV risk behaviors over time. Addictive Behaviors, 29, 567-574.

Kiang, L., Witkow, M. R., Baldelomar, O. A, \& Fuligni, A. J. (2010). Change in ethnic identity across the high school years among adolescents with Latin American, Asian, and European backgrounds. Journal of Youth and Adolescence, 39(6), 683-93. doi:10.1007/s10964-009-9429-5

Lee, M., and Rotheram-Borus, M. J. (2001). Challenges Associated with increased survival among parents living with HIV. American Journal of Public Health, 91, 13031309. doi: 10.2105/AJPH.91.8.1303

Lee, Y. H., Salman, A., \& Fitzpatrick, J. J. (2009). HIV/AIDS preventive self-Efficacy, depressive symptoms, and risky sexual behavior in adolescents: A cross-sectional questionnaire survey. International Journal of Nursing Studies, 46(5), 653-660. doi:10.1016/j.ijnurstu.2008.11.007

Lopez, C. R., Antoni, M. H., Fekete, E. M., \& Penedo, F. J. (2010). Ethnic identity and perceived stress in HIV+ Minority Women: The role of coping self-efficacy and social support. International Journal of Behavioral Medicine. doi: 10.1007/s12529-010-9121

Lowe, D. (1960) Harvest of Shame. CBS News with Edward Murrow. Retrieved from: http://www.cbsnews.com/video/watch/?id=7087479n\&tag=contentMain;contentBody.

Lyyra, T. M. \& Heikkinen, R. L. (2006). Perceived social support and mortality in older people. The Journals of Gerontology, 61(3), S147-52. Retrieved from http://www.ncbi.nlm.nih.gov/pubmed/16670192

Mazzaferro, K. E., Murray, P. J., Ness, R. B., Bass, D. C., Tyus, N., \& Cook, R. L. (2006). Depression, stress, and social support as predictors of high-risk sexual behaviors and STIs in young women. The Journal of Adolescent Health, 39(4), 601-3. doi:10.1016/j.jadohealth.2006.02.004

McCoy, H. V., Hlaing, W. M., Ergon-Rowe, E., Samuels, D., \& Malow, R. (2009). Lessons from the fields: A migrant HIV prevention project. Public Health Reports, 124(6), 790-6. Retrieved from http://www.ncbi.nlm.nih.gov/pmc/articles/PMC2773941/

Motl, R. W., Mcauley, E., Snook, E. M., \& Gliottoni, R. C. (2010). Physical activity and quality of life in multiple sclerosis: Intermediary roles of disability, fatigue, mood, pain, self-efficacy and social support. Psychology and Health, 14(1), 111-124.

doi: $10.1080 / 13548500802241902$

Peterson, H. C., Buser, T. J., \& Westburg, N. G. (2010). Effects of familial attachment, social support, involvement, and self-esteem on youth substance use and sexual risk taking. The Family Journal, 18(4), 369-376. doi:10.1177/1066480710380546 
Phinney, J. S. (1992). The Multigroup Ethnic Identity Measure: A new scale for use with diverse groups. Journal of Adolescent Research, 7(2), 156-176.

doi:10.1177/074355489272003

Phinney, J. S., \& Ong, A. D. (2007). Conceptualization and measurement of ethnic identity: Current status and future directions. Journal of Counseling Psychology, 54(3), 271-281. doi:10.1037/0022-0167.54.3.271

Phinney, J. S., Cantu, C. L., \& Kurtz, D. A. (1997). Ethnic and American identity as predictors of self-esteem among African American, Latino, and White adolescents. Journal of Youth and Adolescence, 26(2), 165-185. doi: 10.1023/A:1024500514834

Phinney, J. S., Horenczyk, G., Liebkind, K., \& Vedder, P. (2001). Ethnic identity, immigration, and well-being: An interactional perspective. Journal of Social Issues, 57(3), 493-510. doi:10.1111/0022-4537.00225

Persky, I. \& Birman, D. (2005). Ethnic identity in acculturation research: a study of multiple identities of Jewish refugees from the former Soviet Union. Journal of Cross Cultural Psychology, 36, 557.

Rhodes, S. D., Bischoff, Ã. W. E., Burnell, J. M., Whalley, L. E., Walkup, M. P., Vallejos ... Arcury, T. A. (2010). HIV and sexually transmitted disease risk among male Hispanic / Latino migrant farmworkers in the Southeast: Findings from a pilot CBPR study. American Journal of Industrial Medicine. doi:10.1002/ajim.20807.

Schneider, M. E., \& Ward, D. J. (2003). The role of ethnic identification and perceived social support in Latinos' adjustment to college. Hispanic Journal of Behavioral Sciences, 25(4), 539-554. doi:10.1177/0739986303259306

Shehadeh, N., McCoy, H. V., Rubens, M. B., Batra, A., Renfrew, R., \& Winter, K. (2011). The impact of ethnic identity on changes in high risk HIV Behaviors in sexually active migrant workers. Journal of Immigrant and Minority Health, 14(1), 100-106. doi: 10.1007/s10903-011-9466-5.

Smith, E. P., Walker, K., Fields, L., Brookins, C. C., \& Seay, R. C. (1999). Ethnic identity and its relationship to self-esteem, perceived self-efficacy and prosocial attitudes in early adolescence. Journal of Adolescence, 22, 867-880.

IBM Corporation. (2010). IBM SPSS Base 19.0 for Windows User's Guide. IBM Corporation, New York.

Susser, E., Desvarieux, M., \& Wittkowski, K. M. (1998). Reporting sexual risk behavior for HIV: A practical risk index and a method for improving risk indices. American Journal of Public Health, 88(4), 671-4. doi: 10.2105/AJPH.88.4.671 
Utsey, S., Chae, M. H., Brown, C. F., \& Kelly, D. (2002). Effect of ethnic group membership on ethnic Identity, race-related stress, and quality of life. Cultural Diversity and Ethnic Minority Psychology, 8(4), 366-377. doi: 10.1037/1099-9809.8.4.367

Weatherby, N. L., McCoy, H. V., Bletzer, K. V., McCoy, C. B., Inciardi, J. A., McBride, D. C., \& Forney, M. A. (1997). Immigration and HIV among migrant workers in rural Southern Florida. Journal of Drug Issues, 27(1), 155-173.

Yoo, H. C., \& Lee R. M. (2005). Ethnic identity and approach-type coping as moderators of the racial discrimination/well-being relation in Asian Americans. Journal of Counseling Psychology, 52,497-506. 


\section{Chapter VII}

\section{OVERALL CONCLUSIONS}

The main purpose of the studies presented in this dissertation was to attain a clearer picture of the role of ethnic identity in relation to HIV risk behaviors. Previous research describes ethnic identity as playing a protective role in adolescent youth regarding drug and alcohol use. Ethnic identity has also shown positive associations with psychological well-being (Umana-Taylor \& Updegraff, 2007) and academic outcomes (Schwartz, Zamboanga, \& Jarvis, 2007). The proposed research questions addressed ethnic identity as also playing a potentially protective role among migrant workers.

The first research question addressed in Manuscript 1 examined ethnic identity as it related to HIV risk behaviors including risky sexual behaviors and drug and alcohol use, as well as migrant workers' beliefs about the effects of alcohol on sexual encounters at baseline. The analyses demonstrated that ethnic identity had an inverse relationship with number of sexual partners without a condom and number of unprotected vaginal acts without a condom. Those with higher levels of ethnic identity had shown lower levels of risky behaviors. These findings demonstrated that there was a protective effect for ethnic identity with this migrant worker sample.

The second research question proposed that increases in ethnic identity from baseline to the 6-month follow-up assessment may be associated with reductions in HIV risk behaviors. Analyses were conducted, in Manuscript 2, to determine if ethnic identity changed from baseline to 6-month follow-up. The analyses revealed that there were changes in ethnic identity; however, these changes became non-significant when demographic covariates were added to the regression equations. Additional analysis 
showed that increases in ethnic identity were associated with decreases in alcohol consumption and decreases in SRAE-Increased Risk score over time. These findings suggest that increases in ethnic identity may contribute to decreased positive beliefs about the effects of alcohol and decreased alcohol consumption.

The third research question, addressed in Manuscript 3, proposed that social support change contributed directly to changes in ethnic identity over time. Social support was also examined as potential moderator in the relationship between ethnic identity and risky HIV behaviors. The analyses exploring the moderator effects yielded no significant results. However, social support were found to be positively associated with changes in ethnic identity. These findings suggest that improvements in social support may positively influence ethnic identity, which is itself related to decreases in risky behaviors. The last research question examined the relationship of the intervention group assignment on changes in ethnic identity. No significant associations were found.

Although the results of these analyses, examining the relationship between ethnic identity and HIV risk behaviors and beliefs, yielded few significant findings, the implications are important. While tentative, the statistically significant findings suggest that interventions to reduce engagement in risky behaviors might show improved outcomes if efforts are made to increase ethnic identity. The purpose of these studies was primarily a stepping stone for further ethnic identity research to be implemented on migrant workers and other samples. Such research initiatives may provide a clearer understanding of how ethnic identity may play a role in HIV risk behaviors as well in gaining a better understanding of ethnic identity in migrant worker communities. 


\section{Limitations}

There were a number of limitations in the studies described in this report. Such limitations were inevitable due to the existing circumstances of the parent project from which these data were obtained. The analyses were conducted after the completion of the HIV prevention intervention, which reduced the ability of the current study to find significant results since the original data were not collected with these research questions or hypotheses in mind. Another limitation was the loss of participants at follow-up assessments. There may be a number of reasons for the loss of participants, but a major reason may be the fear that is common among many migrant workers of being reported to immigration services. One last limitation that must be mentioned is the social desirability bias that is linked with self-reported data. In many cases participants may have reported what they felt was a desirable answer for the interviewer rather than what actually occurred.

\section{Directions for future research}

Migrant workers are a population in desperate need of further HIV risk research. This research should lead to migrant workers being provided with appropriate intervention programs to address the issue of HIV risk. This community tends to be overlooked due to their constant mobility.

There is still much to be done to understand the relationship between ethnic identity and HIV risk. The role of public health researchers and other health professionals must be to better understand the role of ethnic identity and whether it plays a protective role in migrant worker communities. The outcomes of future studies may help to develop prevention programs that are better suited and more effective for this population. 
APPENDICES 
APPENDIX 1: Correlations of Alcohol Use, Drug Use, and Alcohol Expectancy with Demographics 
Table Appendix 1

Correlations of Alcohol Use, Drug Use, and Alcohol Expectancy with Demographics

\begin{tabular}{|c|c|c|c|c|c|c|c|c|c|c|c|c|c|c|}
\hline $\begin{array}{l}\text { Demographic } \\
\text { Variables }\end{array}$ & $\mathrm{AC}$ & MU & $\mathrm{CU}$ & SRAE & $\begin{array}{l}\text { SRAE } \\
\text { - ES }\end{array}$ & $\begin{array}{l}\text { SRAE } \\
\text { - DN }\end{array}$ & $\begin{array}{l}\text { SRAE } \\
\text { - IR }\end{array}$ & $\mathrm{AC} \Delta$ & MU $\Delta$ & $\mathrm{CU} \Delta$ & SRAE $\Delta$ & $\begin{array}{l}\text { SRAE } \\
- \text { ES } \Delta\end{array}$ & $\begin{array}{l}\text { SRAE } \\
-\mathrm{DN} \Delta\end{array}$ & $\begin{array}{l}\text { SRAE } \\
- \text { IR } \Delta\end{array}$ \\
\hline Language & $.177 *$ & $-.091 * *$ & -.056 & .094 & $.105^{* *}$ & .096 & .072 & .001 & .053 & -.036 & -.016 & -.049 & -.044 & .041 \\
\hline Gender & $-.116^{*}$ & $.095 *$ & $.115^{*}$ & $-.175^{*}$ & $-.141 *$ & $-.211 *$ & $-.178^{*}$ & -.039 & .029 & .092 & .030 & .029 & .023 & -.049 \\
\hline Education & $-.130 *$ & $.110 *$ & .018 & $-.130 *$ & $-.180^{*}$ & $-.123^{*}$ & -.009 & -.029 & -.003 & .064 & .090 & .063 & .086 & .060 \\
\hline $\begin{array}{l}\text { Marital } \\
\text { Status }\end{array}$ & .030 & $-.080 * *$ & -.023 & -.078 & -.010 & -.096 & -.065 & $-.152 *$ & .086 & -.055 & .057 & .096 & .007 & .062 \\
\hline Ethnicity & $.140^{*}$ & .023 & .038 & $.144^{*}$ & .108 & $.133^{*}$ & $.147^{*}$ & -.027 & .025 & .094 & -.079 & -.090 & -.082 & .028 \\
\hline $\begin{array}{l}\text { Country of } \\
\text { Birth }\end{array}$ & $-.129 *$ & $.091 *$ & .058 & $.122 * *$ & $.139 *$ & $.102 * *$ & .047 & -.048 & .031 & -.047 & -.040 & -.021 & -.036 & .041 \\
\hline Age & -.039 & $-.284 *$ & -.037 & -.003 & .030 & -.015 & -.024 & -.051 & .006 & .036 & .056 & .071 & .039 & -.018 \\
\hline $\begin{array}{l}\text { Length of } \\
\text { Stay }\end{array}$ & .052 & .055 & .046 & -.088 & -.075 & -.100 & $-.159 *$ & $.132 *$ & .030 & .036 & $.137^{*}$ & $.140^{*}$ & .100 & -.034 \\
\hline
\end{tabular}

Note: $\mathrm{AC}=$ Alcohol Consumption; MU= Marijuana Use; $\mathrm{CU}=$ Crack Use; SRAE- Sex Related Alcohol Expectancy; SRAE-ES= SRAE-Enhanced Sex; SRAE-DN= SRAE- Decreased Nervousness; SRAE-IR= SRAE-Increased Risk; VEE= Vaginal Episode Equivalent; $\mathrm{SP}=$ Sexual Partner; USP= Unprotected Sexual Partners; UVA= Unprotected Vaginal Acts. All Sexual acts and consumption of alcohol and illegal substances refer to the last 30 days.

$* p<.05 * * p .10$ 
APPENDIX 2: Correlations of Risky Sexual Behaviors and Demographics 
Table Appendix 2.1

Correlations of Risky Sexual Behaviors with Demographics

\begin{tabular}{lcccccccc}
\hline \multicolumn{1}{c}{ Demographics } & VEE & SP & USP & UVA & VEE $\Delta$ & SP $\Delta$ & USP $\Delta$ & UVA $\Delta$ \\
\hline Language & $-126^{*}$ & -.034 & -.064 & $-.145^{*}$ & -.024 & .067 & -.028 & $-.104^{*}$ \\
Gender & $.188^{*}$ & $.112^{*}$ & $.114^{*}$ & $.210^{*}$ & -.003 & -.043 & .038 & .024 \\
Education & $.088^{* *}$ & .034 & .018 & $.102^{*}$ & .053 & .008 & .029 & .050 \\
Marital Status & $.114^{*}$ & -.033 & .006 & $.114^{*}$ & $-.111^{*}$ & .002 & .008 & -.083 \\
Ethnicity & .022 & $.043^{*}$ & .004 & $.001^{*}$ & -.011 & -.026 & -.057 & .015 \\
Country of Birth & $.116^{*}$ & .050 & .049 & $.141^{*}$ & -.055 & .025 & .001 & -.089 \\
Age & $-.101^{*}$ & -.059 & -.017 & $-.099^{*}$ & -.024 & -.020 & -.003 & -.057 \\
Length of Stay & .048 & .009 & .030 & .057 & -.036 & $-.122^{*}$ & -.036 & .052
\end{tabular}

Note: $\mathrm{AC}=\mathrm{VEE}=$ Vaginal Episode Equivalent; $\mathrm{SP}=$ Sexual Partner; $\mathrm{USP}=$ Unprotected Sexual Partners; UVA= Unprotected Vaginal Acts. All Sexual acts and consumption of alcohol and illegal substances refer to the last 30 days.

$* p<.05 * * p<.10$ 
APPENDIX 3: Tables on Power for regressions conducted in Manuscripts 1, Manuscript

2, and Manuscript 3 
Table Appendix 3.1

Power analysis for Each Regression Analysis at baseline $(n=431)$ found in Manuscript 1 and 2

\begin{tabular}{lcc}
\hline Outcome variable & $\begin{array}{c}\text { Number of } \\
\text { Predictors } \\
\text { (2 test predictors) }\end{array}$ & $\begin{array}{c}\text { Power Analysis } \\
\text { power }=(1-\beta)\end{array}$ \\
\hline VEE & 8 & 1.0 \\
Unprotected Vaginal acts & 8 & .997 \\
Alcohol & 6 & .734 \\
Crack & 3 & .866 \\
SRAE-Increased Risk & 7 & .913 \\
\hline
\end{tabular}

Table Appendix 3.2

Power analysis for Regressions at 6 month follow-up $(n=270)$ found in Manuscript1

\begin{tabular}{lcc}
\hline Outcome variable & $\begin{array}{c}\text { Number of } \\
\text { predictors } \\
\text { (2 test predictors) }\end{array}$ & $\begin{array}{c}\text { Power analysis- } \\
\text { power }=(1-\beta)\end{array}$ \\
\hline Alcohol $\Delta$ & 5 & .972 \\
SRAE-Increased Risk $\Delta$ & 4 & .762 \\
\hline
\end{tabular}

Table Appendix 3.3

Power analysis for Regressions in Manuscript 3 ( $n=270)$

\begin{tabular}{ccc}
\hline Outcome variable & $\begin{array}{c}\text { Number of } \\
\text { predictors } \\
\text { (2 test predictors) }\end{array}$ & $\begin{array}{c}\text { Power Analysis } \\
\text { power }=(1-\beta)\end{array}$ \\
\hline EI total EI total w/SPS $\Delta$ & 7 & 1.00 \\
$\begin{array}{l}\text { EI Belonging } \\
\text { EI Belonging w/SPS } \Delta\end{array}$ & 7 & .999 \\
$\begin{array}{c}\text { EI Explore } \\
\text { EI Explore w/SPS } \Delta\end{array}$ & 7 & .988 \\
\hline
\end{tabular}

*Note: EI = ethnic identity; SPS = Social Support. 
APPENDIX 4: Tables on Demographics by Ethnicity for the Baseline and 6 Month Follow-up Sample 
Table Appendix 4.1

Demographic Characteristics by Ethnicity at Baseline $(n=431)$

\begin{tabular}{|c|c|c|c|}
\hline Demographics & $\begin{array}{c}\text { African } \\
\text { Americans } \\
(\mathrm{n}=161,37 \%)\end{array}$ & $\begin{array}{c}\text { Hispanics } \\
(\mathrm{n}=270,63 \%)\end{array}$ & $\begin{array}{c}\text { Total } \\
(\mathrm{n}=431,100 \%)\end{array}$ \\
\hline Age (yrs) $($ Mean \pm SD) & $(46.22 \pm 12.24)$ & $(38.79 \pm 11.65)$ & $41.56(12.39)$ \\
\hline Education (yrs) (Mean \pm SD) & $(10.39 \pm 2.66)$ & $(7.30 \pm 3.25)$ & $(8.45+12.39)$ \\
\hline \multicolumn{4}{|l|}{ Gender $n(\%)$} \\
\hline Male & $74,58 \%$ & $106,75 \%$ & $312,72.4 \%$ \\
\hline Female & $54,42 \%$ & $36,25 \%$ & $119,27.6 \%$ \\
\hline \multicolumn{4}{|l|}{ Marital Status $n(\%)$} \\
\hline Single/ Living as Single & $78,61.0 \%$ & $77,54.0 \%$ & $380,81.2 \%$ \\
\hline Married/ Living as Married & $50,39.0 \%$ & $65,46.0 \%$ & $81,18.8 \%$ \\
\hline \multicolumn{4}{|l|}{ Nativity $n(\%)$} \\
\hline US & $161,100 \%$ & $167,61.9 \%$ & $328,76.1 \%$ \\
\hline Mexico & $0,0 \%$ & $103,38.1 \%$ & $103,30.2 \%$ \\
\hline Language $n(\%)$ & $161,100 \%$ & $106,39.3 \%$ & $267,61.9 \%$ \\
\hline Spanish & $0,0 \%$ & $164,60.74 \%$ & $164,38.1 \%$ \\
\hline \multicolumn{4}{|l|}{ Length of Time $n(\%)$} \\
\hline $\begin{array}{l}\text { Longtimers: } \\
\text { (Living in the Immokalee } \leq 5 \text { years) }\end{array}$ & $145,90.1 \%$ & $159,58.9 \%$ & $304,70.5 \%$ \\
\hline $\begin{array}{r}\text { Newcomers: } \\
\text { (Living in the Immokalee }>5 \text { years) }\end{array}$ & $16,9.9 \%$ & $111,41.1 \%$ & $127,29.5 \%$ \\
\hline
\end{tabular}

Table Appendix 4.2

Demographic Characteristics by Ethnicity at Follow-up $(n=270)$

\begin{tabular}{|c|c|c|c|}
\hline Demographics & $\begin{array}{c}\text { African } \\
\text { Americans } \\
(n=128,47 \%) \\
\end{array}$ & $\begin{array}{c}\text { Hispanics } \\
(n=142,53 \%)\end{array}$ & $\begin{array}{c}\text { Total } \\
(n=270,100 \%)\end{array}$ \\
\hline Age (yrs) $(M \pm S D)$ & $(46.89 \pm 12.81)$ & $(38.54+11.38)$ & $42.50(12.76)$ \\
\hline Education (yrs) $(M \pm S D)$ & $(10.57 \pm 2.55)$ & $(7.94 \pm 3.04)$ & $(9.19 \pm 3.1)$ \\
\hline $\begin{array}{r}\text { Male } \\
\text { Female }\end{array}$ & $\begin{array}{l}74,58 \% \\
54,42 \%\end{array}$ & $\begin{array}{c}106,75 \% \\
36,25 \%\end{array}$ & $\begin{array}{c}180,67 \% \\
90,33 \%\end{array}$ \\
\hline $\begin{array}{l}\text { Marital Status } n(\%) \\
\text { Single/ Living as Single } \\
\text { Married/ Living as Married }\end{array}$ & $\begin{array}{l}78,61 \% \\
50,39 \%\end{array}$ & $\begin{array}{l}77,54 \% \\
65,46 \%\end{array}$ & $\begin{array}{l}155,57 \% \\
115,43 \%\end{array}$ \\
\hline Nativity $n(\%)$ & $\begin{array}{c}128,100 \% \\
0,0 \%\end{array}$ & $\begin{array}{l}70,49 \% \\
72,51 \%\end{array}$ & $\begin{array}{c}198,73 \% \\
27 \%\end{array}$ \\
\hline Language $n(\%)$ & $\begin{array}{c}128,100 \% \\
0,0 \%\end{array}$ & $\begin{array}{l}70,49 \% \\
72,51 \%\end{array}$ & $\begin{array}{c}198,73 \% \\
72,26 \%\end{array}$ \\
\hline $\begin{array}{l}\text { Length of Time } n(\%) \\
\text { (Living in the Immokalee } \leq 5 \text { years) }\end{array}$ & $11,8 \%$ & $40,28 \%$ & $51,19 \%$ \\
\hline $\begin{array}{l}\text { Newcomers: } \\
\text { (Living in the Immokalee }>5 \text { years) }\end{array}$ & $117,92 \%$ & $102,72 \%$ & $219,81 \%$ \\
\hline
\end{tabular}


APPENDIX 5: Supplementary Tables for Regressions not reported in Manuscript 1 
Table Appendix 5.1

Multiple Linear Regression Analysis for Predicting Marijuana Usage at Baseline*

Model $1 \quad$ Model 2

\begin{tabular}{lcccccc}
\hline Variable & $B$ & $S E$ & $\beta$ & $B$ & $S E$ & $\beta$ \\
\hline Language & .000 & .114 & .000 & .002 & .114 & .002 \\
Gender & -.150 & .068 & -.123 & -.151 & .069 & $-.124^{*}$ \\
Education & -.002 & .010 & -.014 & -.002 & .010 & -.012 \\
Age & -.009 & .002 & -.204 & -.009 & .002 & $-.203^{*}$ \\
Country of Birth & .007 & .116 & .006 & .004 & .116 & .003 \\
Marital & -.025 & .069 & -.018 & -.023 & .069 & -.017 \\
EI belonging & & & & -.015 & .076 & -.012 \\
EI explore & & & & -.019 & .073 & -.015 \\
\hline
\end{tabular}

Note: EI= Ethnic Identity. All Sexual acts and consumption of alcohol and illegal substances refer to the last 30 days. Log 10 transformation was implemented on Marijuana use variable.

${ }^{*} \mathrm{p} \leq 0.05 ; n=341$; Final block, $F(8,422)=2.766, p=.005$.

Table Appendix 5.2

Multiple Linear Regression Analysis for Predicting SRAE at Baseline

\begin{tabular}{lcccccc}
\hline & \multicolumn{3}{c}{ Model 1 } & \multicolumn{3}{c}{ Model 2 } \\
\hline Variable & $\mathrm{B}$ & $\mathrm{SE}$ & $\beta$ & $\mathrm{B}$ & $\mathrm{SE}$ & $\beta$ \\
\hline Language & -.009 & .168 & -.005 & -.028 & .167 & -.017 \\
Gender & -.247 & .097 & $-.139^{*}$ & -.238 & .096 & $-.133^{*}$ \\
Education & -.021 & .014 & -.091 & -.024 & .014 & -.101 \\
Ethnicity & .204 & .101 & $.124^{*}$ & .213 & .101 & $.129^{*}$ \\
Country of Birth & .067 & .170 & .041 & .088 & .169 & .054 \\
EI belonging & & & & .167 & .109 & .089 \\
EI explore & & & & .129 & .106 & .071 \\
\hline
\end{tabular}

Note: EI= Ethnic Identity. All Sexual acts and consumption of alcohol and illegal substances refer to the last 30 days.

${ }^{*} \mathrm{p} \leq 0.05 ; n=341$; Final block, $F(7,423)=4.701, p<.001$ 
Table Appendix 5.3

Multiple Linear Regression Analysis for Predicting SRAE-Enhanced Sex at Baseline* Model 1 Model 2

\begin{tabular}{lcccccc}
\hline Variable & $B$ & $S E$ & $\beta$ & $B$ & $S E$ & $\beta$ \\
\hline Language & -.072 & .184 & -.040 & -.094 & -.184 & -.052 \\
Gender & -.128 & .106 & -.065 & -.117 & .105 & -.060 \\
Education & -.034 & .016 & $-.132^{*}$ & -.037 & .015 & $-.143^{*}$ \\
Ethnicity & .168 & .111 & .093 & .179 & .110 & .099 \\
Country of Birth & -.073 & .186 & -.041 & -.049 & .185 & -.028 \\
EI belonging & & & & .139 & .116 & .070 \\
EI explore & & & & .191 & .120 & .093 \\
\hline
\end{tabular}

Note: EI= Ethnic Identity. All Sexual acts and consumption of alcohol and illegal substances refer to the last 30 days.

$* \mathrm{p} \leq 0.05 ; n=341$; Final block, $F(7,423)=4.600, p<.001$

Table Appendix 5.4

Multiple Linear Regression Analysis for Predicting SRAE - Decreased Nervousness at Baseline*

\begin{tabular}{lcccccc}
\hline & \multicolumn{3}{c}{ Model 1 } & \multicolumn{5}{c}{ Model 2 } \\
\hline Variable & $B$ & $S E$ & $\beta$ & $B$ & $S E$ & $\beta$ \\
\hline Language & .051 & .196 & .027 & .026 & .196 & .014 \\
Gender & -.368 & .113 & $-.177^{*}$ & -.360 & .112 & $-.173^{*}$ \\
Education & -.020 & .016 & -.073 & -.023 & .017 & -.084 \\
Ethnicity & .225 & .118 & .117 & .234 & .118 & $.122^{*}$ \\
Country of Birth & .179 & .198 & .094 & .199 & .197 & .104 \\
EI belonging & & & & .084 & .124 & .039 \\
EI explore & & & & .207 & .128 & .095 \\
\hline
\end{tabular}

Note: EI= Ethnic Identity. All Sexual acts and consumption of alcohol and illegal substances refer to the last 30 days.

$* \mathrm{p} \leq 0.05 ; n=341$; Final block, $F(7,423)=4.408, p<.001$ 
Table Appendix 5.5

Regression Analysis for Variables Predicting Marijuana Use Change $(n=270)$

\begin{tabular}{lcccccc}
\hline & \multicolumn{3}{c}{ Model 1 } & \multicolumn{3}{c}{ Model 2 } \\
\hline Variable & $\mathrm{B}$ & $S E$ & $\beta$ & $\mathrm{B}$ & $S E$ & $\beta$ \\
\hline Length of Stay & .058 & .124 & .056 & .093 & .126 & .090 \\
Marital & -.140 & .122 & -.138 & -.161 & .124 & -.160 \\
Education & .042 & .017 & $.306^{*}$ & .044 & .017 & $.323^{*}$ \\
Intervention & .051 & .122 & .052 & .020 & .123 & .020 \\
EI belonging $\Delta$ & & & & .212 & .192 & .138 \\
EI explore $\Delta$ & & & & -.299 & .224 & -.163 \\
\hline
\end{tabular}

Note: $\mathrm{EIb}=$ Ethnic Identity belonging; EIe= Ethnic identity explore.

$n=270 * * ; p \leq 0.05, * * p \leq .10$ (trend setting)

Table Appendix 5.6

Regression Analysis for Variables Predicting Crack Use Change $(n=270)$ Model $1 \quad$ Model 2

\begin{tabular}{lcccccc}
\hline Variable & $\mathrm{B}$ & $S E$ & $\beta$ & $\mathrm{B}$ & $S E$ & $\beta$ \\
\hline Education & .276 & .506 & .035 & .362 & .505 & .046 \\
Gender & 4.160 & 3.311 & .080 & 4.616 & 3.311 & .089 \\
Intervention & -3.224 & 3.017 & -.065 & -3.995 & 3.021 & -.080 \\
EI belonging $\Delta$ & & & & 5.299 & 2.438 & .147 \\
EI explore $\Delta$ & & & & -.065 & 2.718 & -.065 \\
\hline
\end{tabular}

Note: $\mathrm{EIb}=$ Ethnic Identity belonging; EIe $=$ Ethnic identity explore. $n=270 * ; * p \leq 0.05, * * p \leq .10$ (trend setting)

Table Appendix 5.7

Regression Analysis for Variables Predicting SRAE Total Change $(\mathrm{n}=270)$

\begin{tabular}{lcccccc}
\hline & \multicolumn{3}{c}{ Model 1 } & & \multicolumn{3}{c}{ Model 2 } \\
\hline Variable & $\mathrm{B}$ & $S E$ & $\beta$ & $\mathrm{B}$ & $S E$ & $\beta$ \\
\hline Education & .025 & .018 & .085 & .023 & .019 & .079 \\
Length of Stay & .171 & .140 & .075 & .152 & .140 & .067 \\
Intervention & .133 & .113 & .072 & .144 & .114 & .078 \\
EI belonging $\Delta$ & & & & -.067 & .092 & -.050 \\
EI explore $\Delta$ & & & & -.085 & .103 & -.056 \\
\hline
\end{tabular}

Note: $\mathrm{EIb}=$ Ethnic Identity belonging; EIe= Ethnic identity explore.

$* p \leq 0.05, * * p \leq .10$ (trend setting)

Table Appendix 5.8 
Regression Analysis for Variables Predicting SRAE-Enhanced Sex Change ( $n=270)$

\begin{tabular}{lcccccc}
\hline & \multicolumn{3}{c}{ Model 1 } & \multicolumn{3}{c}{ Model 2 } \\
\hline Variable & $\mathrm{B}$ & $S E$ & $\beta$ & $\mathrm{B}$ & $S E$ & $\beta$ \\
\hline Length of Stay & .232 & .159 & .089 & .214 & .160 & .082 \\
Intervention & .155 & .130 & .072 & .165 & .131 & .077 \\
EI belonging $\Delta$ & & & & -.064 & .106 & -.041 \\
EI explore $\Delta$ & & & & -.069 & .119 & -.039 \\
\hline
\end{tabular}

Note: $\mathrm{EIb}=$ Ethnic Identity belonging; EIe= Ethnic identity explore.

$* p \leq 0.05, * * p \leq .10$ (trend setting)

Table Appendix 5.9

Regression Analysis for Variables Predicting SRAE - Decreased Nervousness Change Model 1 Model 2

\begin{tabular}{lcccccc}
\hline Variable & $\mathrm{B}$ & $S E$ & $\beta$ & $\mathrm{B}$ & $S E$ & $\beta$ \\
\hline Length of Stay & .166 & .075 & .266 & .162 & .075 & .261 \\
Intervention & .096 & .069 & .170 & .121 & .068 & .214 \\
EI belonging & & & & -.090 & .104 & -.109 \\
EI explore & & & & -.257 & .126 & $-.250^{*}$ \\
\hline
\end{tabular}

Note: $\mathrm{EIb}=$ Ethnic Identity belonging; EIe $=$ Ethnic identity explore.

$n=270 ;{ }^{*} p \leq 0.05,{ }^{* *} p \leq .10$ (trend setting) 
APPENDIX 6: Supplementary Tables for Regressions not reported in Manuscript 2 
Table 2.4

Multiple Linear Regression Analysis for Predicting Number of Partners at Baseline $(n=341)$

\begin{tabular}{lcccccc}
\hline & \multicolumn{3}{c}{ Model 1 } & \multicolumn{3}{c}{ Model 2 } \\
\hline Variable & $\beta$ & $S E$ & $B$ & $\beta$ & $S E$ & $B$ \\
\hline Gender & .029 & .027 & .053 & .027 & .027 & .048 \\
EI belonging & & & & -.025 & .034 & -.044 \\
EI explore & & & & -.009 & .035 & -.016 \\
\hline
\end{tabular}

Note: EI= Ethnic Identity. All Sexual acts and consumption of alcohol and illegal substances refer to the last 30 days.

$* p \leq 0.05$; Final block, $F(3,427)=.836, p=.475$

Table 2.5

Regression with Number of Unprotected Sexual Partners

\begin{tabular}{lcccccc}
\hline & \multicolumn{3}{c}{ Model 1 } & \multicolumn{3}{c}{ Model 2 } \\
\hline Variable & $B$ & $S E$ & $\beta$ & $B$ & $S E$ & $\beta$ \\
\hline Gender & .734 & .307 & .115 & .697 & .308 & .108 \\
Marital & .104 & .352 & .014 & .151 & .354 & .021 \\
EI belonging & & & & -.033 & .397 & -.005 \\
EI explore & & & & -.411 & .388 & -.063 \\
\hline
\end{tabular}

Note: EI= Ethnic Identity. All Sexual acts and consumption of alcohol and illegal substances refer to the last 30 days.

$* p \leq 0.05 ; n=341$; Final block, $F(4,426)=1.893, p=.111$.

Table 2.6

Regression Analysis for Predicting Vaginal Episode Equivalent with Change $(n=270)$ Model 1

Model 2

\begin{tabular}{lcccccc}
\hline Variable & $B$ & $S E$ & $\beta$ & $B$ & $S E$ & $\beta$ \\
\hline Marital & -2.421 & 1.327 & $-.111^{* *}$ & -2.440 & 1.347 & -.112 \\
Intervention & .327 & 1.318 & .015 & .562 & 1.328 & .026 \\
EIb $\Delta$ & & & & -1.539 & 1.068 & -.097 \\
EIe $\Delta$ & & & & .460 & 1.214 & .026 \\
\hline
\end{tabular}

Note: $\mathrm{EIb}=$ Ethnic Identity belonging; EIe $=$ Ethnic identity explore.

${ }^{*} p \leq 0.05, * * p \leq .10$ (trend setting) 
Table 2.7

Regression Analysis for Predicting Partner change $(n=270)$

\begin{tabular}{lcccccc}
\hline & \multicolumn{3}{c}{ Model 1 } & \multicolumn{3}{c}{ Model 2 } \\
\hline Variable & $B$ & $S E$ & $\beta$ & $B$ & $S E$ & $\beta$ \\
\hline Length of Stay & -2.027 & 1.093 & $-.112^{* *}$ & -2.094 & 1.101 & -.116 \\
Intervention & -1.455 & .892 & -.099 & -1.362 & .901 & -.092 \\
EI belonging $\Delta$ & & & & -.593 & .725 & -.055 \\
EI explore $\Delta$ & & & & .012 & .816 & .001 \\
\hline
\end{tabular}

Note: EIb= Ethnic Identity belonging; EIe= Ethnic identity explore.

$p \leq 0.05, * * p \leq .10$ (trend setting)

Table 2.8

Regression Analysis for Variables Predicting Unprotected Partners change $(n=270)$

\begin{tabular}{lcccccc}
\hline & \multicolumn{3}{c}{ Model 1 } & \multicolumn{5}{c}{ Model 2 } \\
\hline Variables & $B$ & $S E$ & $\beta$ & $B$ & $S E$ & $\beta$ \\
\hline Length of Stay & -.114 & .273 & -.026 & -.064 & .272 & -.015 \\
Education & -.003 & .040 & -.005 & -.006 & .039 & -.010 \\
Gender & .118 & .239 & .032 & .119 & .238 & .032 \\
Marital & .035 & .218 & .010 & .107 & .220 & .030 \\
Ethnicity & -.199 & .240 & -.057 & -.185 & .238 & -.053 \\
Intervention & -.206 & .216 & -.059 & -.167 & .216 & -.048 \\
EIb $\Delta$ & & & & -.298 & .174 & -.116 \\
EIe $\Delta$ & & & & .458 & .198 & .158 \\
\hline
\end{tabular}

$n=270 ; * p \leq 0.05, * * p \leq .10$ (trend setting)

Table 2.9

Regression Analysis for Variables Predicting Vaginal Acts Without a Condom $(n=270)$ Model 1 Model 2

\begin{tabular}{lcccccc}
\hline Variable & $B$ & $S E$ & $\beta$ & $B$ & $S E$ & $\beta$ \\
\hline Education & .002 & .167 & .001 & .014 & .166 & .007 \\
Country of Birth & -1.012 & 1.258 & -.065 & -.836 & 1.262 & -.054 \\
Length of Stay & .809 & 1.170 & .047 & 1.207 & 1.181 & .069 \\
Intervention & 1.103 & .854 & .080 & 1.065 & .855 & .077 \\
EI belonging $\Delta$ & & & & -.167 & .692 & -.017 \\
EI explore $\Delta$ & & & & 1.557 & .792 & .135 \\
\hline
\end{tabular}

Note: $\mathrm{EIb}=$ Ethnic Identity belonging; EIe $=$ Ethnic identity explore. $n=270 ; * p \leq 0.05, * * p \leq .10$ (trend setting) 
APPENDIX 7: Correlation table for Manuscript 3 
Table Appendix 7.1

Correlations of Change in Risky Sexual Behaviors, Drug Use, Alcohol Use, and Alcohol Expectancy Measure

\begin{tabular}{lccccccccccc}
\hline & & & & & & & & & & & \\
Demographic & $\mathrm{AC}$ & $\mathrm{MU}$ & $\mathrm{CU}$ & $\mathrm{SRAE}$ & $\mathrm{SRAE}$ & $\mathrm{SRAE}$ & \multicolumn{2}{c}{ SRAE } & VEE & SP & \multicolumn{2}{c}{ USP } & \multicolumn{2}{c}{ UVA } \\
\multicolumn{1}{c}{ Variables } & $\Delta$ & $\Delta$ & $\Delta$ & $\Delta$ & $-\mathrm{ES} \Delta$ & $-\mathrm{DN} \Delta$ & $-\mathrm{IR} \Delta$ & $\Delta$ & $\Delta$ & $\Delta$ & $\Delta$ \\
\hline Language & .001 & .053 & -.036 & -.016 & -.049 & -.044 & .041 & -.024 & .067 & -.028 & $-.104^{*}$ \\
Gender & -.039 & .029 & .092 & .030 & .029 & .023 & -.049 & -.003 & -.043 & .038 & .024 \\
Education & -.029 & -.003 & .064 & .090 & .063 & .086 & .060 & .053 & .008 & .029 & .050 \\
Marital & $-.152^{*}$ & .086 & -.055 & .057 & .096 & .007 & .062 & $-.111^{*}$ & .002 & .008 & -.083 \\
Status & & & & & & & & & & & \\
Ethnicity & -.027 & .025 & .094 & -.079 & -.090 & -.082 & .028 & -.011 & -.026 & -.057 & .015 \\
Country of & -.048 & .031 & -.047 & -.040 & -.021 & -.036 & .041 & -.055 & .025 & .001 & -.089 \\
Birth & & & & & & & & & & & \\
Age & -.051 & .006 & .036 & .056 & .071 & .039 & -.018 & -.024 & -.020 & -.003 & -.057 \\
Length of & $.132 *$ & .030 & .036 & $.137^{*}$ & $.140 *$ & .100 & -.034 & -.036 & $-.122 *$ & -.036 & .052 \\
Stay & & & & & & & & & & &
\end{tabular}

Note: $\mathrm{AC}=$ Alcohol Consumption; $\mathrm{MU}=$ Marijuana Use; $\mathrm{CU}=$ Crack Use; SRAE- Sex Related Alcohol Expectancy; SRAE-ES= SRAE-Enhanced Sex; SRAE-DN= SRAEDecreased Nervousness; SRAE-IR= SRAE-Increased Risk; VEE= Vaginal Episode Equivalent; $\mathrm{SP}=$ Sexual Partner; USP $=$ Unprotected Sexual Partners; UVA= Unprotected Vaginal Acts. All Sexual acts and consumption of alcohol and illegal substances refer to the last 30 days. ${ }^{*} p<.05 * * p<.1 .0$ 
APPENDIX 8: Supplementary Tables for Regressions not reported in Manuscript 3 
Table Appendix 8.1

Regression Analysis for Moderating Variables Predicting Vaginal Episode Equivalent Change

\begin{tabular}{|c|c|c|c|c|c|c|c|c|c|c|c|c|}
\hline \multirow[b]{2}{*}{ Variable } & \multicolumn{3}{|c|}{ Model 1} & \multicolumn{3}{|c|}{ Model 2} & \multicolumn{3}{|c|}{ Model 3} & \multicolumn{3}{|c|}{ Model 4} \\
\hline & $\beta$ & $S E$ & $B$ & $\beta$ & $S E$ & $B$ & $\beta$ & $S E$ & $B$ & $\beta$ & $S E$ & $B$ \\
\hline Marital & -2.421 & 1.327 & -.111 & -2.480 & 1.332 & -.114 & -2.477 & 1.338 & -.114 & -2.490 & 1.340 & -.114 \\
\hline Intervention & .327 & 1.318 & .015 & .57 & 1.321 & .016 & .383 & 1.328 & .018 & .284 & 1.342 & .013 \\
\hline EI $\Delta$ & & & & .750 & 1.223 & -.037 & -.754 & 1.379 & -.038 & -.869 & 1.383 & -.043 \\
\hline$z-\operatorname{SPS} \Delta$ & & & & & & & -.093 & .746 & -.009 & -.273 & .774 & -.025 \\
\hline $\mathrm{EI} \Delta \mathrm{X} \operatorname{SPS} \Delta$ & & & & & & & & & & .824 & .688 & .077 \\
\hline
\end{tabular}

Note: EI= Ethnic Identity; SPS= Social Provisions Scale. All Sexual acts and consumption of alcohol and illegal substances refer to the last 30 days.

$* p \leq 0.05 ; n=270$

Table Appendix 8.2

Regression Analysis for Moderating Variables Predicting Partner Change

\begin{tabular}{|c|c|c|c|c|c|c|c|c|c|c|c|c|}
\hline \multirow[b]{2}{*}{ Variable } & \multicolumn{3}{|c|}{ Model 1} & \multicolumn{3}{|c|}{ Model 2} & \multicolumn{3}{|c|}{ Model 2} & \multicolumn{3}{|c|}{ Model 4} \\
\hline & $\beta$ & $S E$ & $B$ & $\beta$ & $S E$ & $B$ & $\beta$ & $S E$ & $B$ & $\beta$ & $S E$ & $B$ \\
\hline Length of Stay & -2.027 & 1.093 & -.112 & -2.113 & 1.097 & -.117 & -2.010 & 1.101 & -.111 & -1.968 & 1.110 & -.109 \\
\hline EI $\Delta$ & & & & -.717 & .828 & -.053 & -.731 & .925 & -.054 & -.707 & .930 & -.052 \\
\hline z- SPS $\Delta$ & & & & & & & -.260 & .502 & -.035 & -.201 & .523 & -.027 \\
\hline EI $\Delta X z-\operatorname{SPS} \Delta$ & & & & & & & & & & -.194 & .465 & -.027 \\
\hline
\end{tabular}

Note: EI= Ethnic Identity; SPS= Social Provisions Scale. All Sexual acts and consumption of alcohol and illegal substances refer to the last 30 days.

$* p \leq 0.05 ; n=270$ 
Table Appendix 8.3

Multiple Linear Regression Analysis for Moderating Variables Predicting Unprotected Partner Change

\begin{tabular}{|c|c|c|c|c|c|c|c|c|c|c|c|c|}
\hline & \multicolumn{3}{|c|}{ Model 1} & \multicolumn{3}{|c|}{ Model 2} & \multicolumn{3}{|c|}{ Model 3} & \multicolumn{3}{|c|}{ Model 4} \\
\hline Variable & $\beta$ & $S E$ & $B$ & $\beta$ & $S E$ & $B$ & $\beta$ & $S E$ & $B$ & $\beta$ & $S E$ & $B$ \\
\hline Length of Stay & -.043 & .262 & -.010 & -.037 & .263 & -.009 & .016 & .262 & .004 & .037 & .262 & .009 \\
\hline EI $\Delta$ & & & & .051 & .199 & .016 & -.028 & .220 & -.009 & .001 & .220 & .000 \\
\hline$z-\operatorname{SPS} \Delta$ & & & & & & & -.006 & .119 & -.003 & .032 & .124 & .018 \\
\hline EI $\Delta X z-\operatorname{SPS} \Delta$ & & & & & & & & & & -.193 & .110 & $-.112 * *$ \\
\hline
\end{tabular}

Note: EI= Ethnic Identity; SPS= Social Provisions Scale. All Sexual acts and consumption of alcohol and illegal substances refer to the last 30 days. ${ }^{*} p \leq 0.05 ; n=270$

Table Appendix 8.4

Multiple Linear Regression Analysis for Moderating Variables Predicting Unprotected Vaginal Acts Change

\begin{tabular}{|c|c|c|c|c|c|c|c|c|c|c|c|c|}
\hline & \multicolumn{3}{|c|}{ Model 1} & \multicolumn{3}{|c|}{ Model 2} & \multicolumn{3}{|c|}{ Model 3} & \multicolumn{3}{|c|}{ Model 4} \\
\hline Variable & $\beta$ & $S E$ & $B$ & $\beta$ & $S E$ & $B$ & $\beta$ & $S E$ & $B$ & $\beta$ & $S E$ & $B$ \\
\hline Gender & .127 & .239 & .034 & .136 & .240 & .037 & .231 & .241 & .062 & .247 & .240 & .067 \\
\hline Marital & .030 & .218 & .009 & .037 & .219 & .011 & .039 & .216 & .011 & .042 & .216 & .012 \\
\hline Education & .009 & .037 & .017 & .010 & .037 & .018 & .017 & .036 & .030 & .011 & .036 & .020 \\
\hline Length of Stay & -.078 & .269 & -.018 & -.071 & .270 & -.017 & -.037 & .268 & -.009 & -.013 & .268 & -.003 \\
\hline Intervention & -.209 & .216 & -.060 & -.211 & .216 & -.060 & -.186 & .214 & -.053 & -.155 & .215 & -.044 \\
\hline $\mathrm{EI} \Delta$ & & & & .073 & .202 & .023 & -.009 & .222 & -.003 & .020 & .222 & .006 \\
\hline$z-\operatorname{SPS} \Delta$ & & & & & & & .007 & .121 & .004 & .044 & .125 & .025 \\
\hline $\mathrm{EI} \Delta \mathrm{X} z-\operatorname{SPS} \Delta$ & & & & & & & & & & -.192 & .110 & -.111 \\
\hline
\end{tabular}

Note: EI= Ethnic Identity; SPS= Social Provisions Scale. All Sexual acts and consumption of alcohol and illegal substances refer to the last 30 days. ${ }^{*} p \leq 0.05 ; n=270$ 
Table Appendix 8.5

Regression Analysis for Moderating Variables Predicting Monthly Alcohol Consumption

\begin{tabular}{|c|c|c|c|c|c|c|c|c|c|c|c|c|}
\hline & \multicolumn{3}{|c|}{ Model 1} & \multicolumn{3}{|c|}{ Model 2} & \multicolumn{3}{|c|}{ Model 3} & \multicolumn{3}{|c|}{ Model 4} \\
\hline Variable & $\beta$ & $S E$ & $B$ & $\beta$ & $S E$ & $B$ & $\beta$ & $S E$ & $B$ & $\beta$ & $S E$ & $B$ \\
\hline Length of Stay & 26.090 & 12.043 & .131 & 30.051 & 11.808 & .151 & 28.790 & 11.805 & .145 & 28.548 & 11.899 & .144 \\
\hline EI $\Delta$ & & & & 33.292 & 8.906 & .222 & 41.718 & 9.916 & .279 & 41.941 & 9.971 & $.280 * *$ \\
\hline $\mathrm{Z}-\operatorname{SPS} \Delta$ & & & & & & & -11.554 & 5.383 & -.143 & -11.725 & 5.610 & -.145 \\
\hline EI $\Delta \mathrm{XZ}-\operatorname{SPS} \Delta$ & & & & & & & & & & -.821 & 4.988 & -.010 \\
\hline
\end{tabular}

Note: EI= Ethnic Identity; SPS= Social Provisions Scale. All Sexual acts and consumption of alcohol and illegal substances refer to the last 30 days.

$* p \leq 0.05 ; n=270$

Table Appendix 8.6

Regression Analysis for Moderating Variables Predicting Marijuana Usage Change

\begin{tabular}{|c|c|c|c|c|c|c|c|c|c|c|c|c|}
\hline \multirow[b]{2}{*}{ Variable } & \multicolumn{3}{|c|}{ Model 1} & \multicolumn{3}{|c|}{ Model 2} & \multicolumn{3}{|c|}{ Model 3} & \multicolumn{3}{|c|}{ Model 4} \\
\hline & $\beta$ & $S E$ & $B$ & $\beta$ & $S E$ & $B$ & $\beta$ & $S E$ & $B$ & $\beta$ & $S E$ & $B$ \\
\hline Marital & 3.319 & 2.297 & .089 & 3.226 & 2.309 & .086 & 3.289 & 2.315 & .088 & 3.299 & 2.324 & .088 \\
\hline Education & .062 & .373 & .010 & .044 & .375 & .007 & .003 & .378 & .000 & -.005 & .381 & -.001 \\
\hline Length of Stay & .472 & 2.822 & .010 & .370 & 2.835 & .008 & .135 & 2.849 & .003 & .118 & 2.873 & .003 \\
\hline Intervention & 2.323 & 2.280 & .063 & 2.350 & 2.284 & .063 & 2.364 & 2.293 & .064 & 2.454 & 2.322 & .066 \\
\hline EI $\Delta$ & & & & -.978 & 2.124 & -.029 & .000 & 2.376 & .000 & .029 & 2.389 & .001 \\
\hline$z-\operatorname{SPS} \Delta$ & & & & & & & -.967 & 1.286 & -.052 & -.985 & 1.340 & -.053 \\
\hline $\mathrm{EI} \Delta \mathrm{X} z-\operatorname{SPS} \Delta$ & & & & & & & & & & -.121 & 1.190 & -.007 \\
\hline
\end{tabular}

Note: EI= Ethnic Identity; SPS= Social Provisions Scale. All Sexual acts and consumption of alcohol and illegal substances refer to the last 30 days. $^{*} p \leq 0.05 ; n=270$ 
Table Appendix 8.7

Regression Analysis for Moderating Variables Predicting Crack Usage Change

\begin{tabular}{|c|c|c|c|c|c|c|c|c|c|c|c|c|}
\hline & \multicolumn{3}{|c|}{ Model 1} & \multicolumn{3}{|c|}{ Model 2} & \multicolumn{3}{|c|}{ Model 3} & \multicolumn{3}{|c|}{ Model 4} \\
\hline Variable & $\beta$ & $S E$ & $B$ & $\beta$ & $S E$ & $B$ & $\beta$ & $S E$ & $B$ & $\beta$ & $S E$ & $B$ \\
\hline Marital & -2.546 & 3.051 & -.051 & -2.483 & 3.068 & -.050 & -2.448 & 3.076 & -.049 & -2.474 & 3.085 & -.050 \\
\hline Education & .426 & .496 & .054 & .438 & .499 & .055 & .484 & .503 & .061 & .512 & .506 & .065 \\
\hline Length of Stay & -.314 & 3.749 & -.005 & -.245 & 3.767 & -.004 & -.048 & 3.786 & -.001 & -.098 & 3.814 & -.002 \\
\hline Intervention & -3.278 & 3.029 & -.066 & -3.296 & 3.035 & -.067 & -3.136 & 3.047 & -.064 & -3.372 & 3.083 & -.068 \\
\hline EI $\Delta$ & & & & .661 & 2.823 & .015 & .462 & 3.157 & .010 & .333 & 3.172 & .007 \\
\hline$z$-SPS $\Delta$ & & & & & & & -.292 & 1.708 & -.012 & -.368 & 1.779 & -.015 \\
\hline EI $\Delta \mathrm{X} z$-SPS $\Delta$ & & & & & & & & & & .757 & 1.581 & .031 \\
\hline
\end{tabular}

Note: EI= Ethnic Identity; SPS= Social Provisions Scale. All Sexual acts and consumption of alcohol and illegal substances refer to the last 30 days. ${ }^{*} p \leq 0.05 ; n=270$

Table Appendix 8.8

Regression Analysis for Moderating Variables Predicting SRAE total Change

\begin{tabular}{|c|c|c|c|c|c|c|c|c|c|c|c|c|}
\hline & \multicolumn{3}{|c|}{ Model 1} & \multicolumn{3}{|c|}{ Model 2} & \multicolumn{3}{|c|}{ Model 3} & \multicolumn{3}{|c|}{ Model 4} \\
\hline Variable & $\beta$ & $S E$ & $B$ & $\beta$ & $S E$ & $B$ & $\beta$ & $S E$ & $B$ & $\beta$ & $S E$ & $B$ \\
\hline Gender & .034 & .121 & .017 & .017 & .122 & .009 & .031 & .124 & .016 & .030 & .124 & .016 \\
\hline Length of Stay & .196 & .140 & .087 & .185 & .140 & .082 & .189 & .141 & .083 & .202 & .142 & .089 \\
\hline Intervention & .119 & .113 & .064 & .124 & .113 & .067 & .130 & .113 & .070 & .131 & .115 & .071 \\
\hline $\mathrm{EI} \Delta$ & & & & -.120 & .105 & -.070 & -.112 & .117 & -.065 & -.103 & .118 & -.060 \\
\hline$z$-SPS $\Delta$ & & & & & & & -.029 & .064 & -.031 & -.009 & .066 & -.010 \\
\hline EI $\Delta \mathrm{X} z$-SPS $\Delta$ & & & & & & & & & & -.071 & .059 & -.077 \\
\hline
\end{tabular}

Note: EI= Ethnic Identity; SPS= Social Provisions Scale. All Sexual acts and consumption of alcohol and illegal substances refer to the last 30 days. ${ }^{*} p \leq 0.05 ; n=270$ 
Table Appendix 8.9

Regression Analysis for Moderating Variables Predicting SRAE-Enhanced Sex Change

\begin{tabular}{lccccccccccccc}
\hline & \multicolumn{3}{c}{ Model 1 } & \multicolumn{1}{c}{ Model 2 } & \multicolumn{1}{c}{ Model 3 } \\
\hline Variable & $\beta$ & $S E$ & $B$ & $\beta$ & $S E$ & $B$ & $\beta$ & $S E$ & $B$ & $\beta$ & $S E$ & $B$ \\
\hline Gender & .040 & .139 & .018 & .021 & .140 & .009 & .047 & .143 & .021 & .046 & .143 & .020 \\
Length of Stay & .224 & .162 & .086 & .212 & .162 & .081 & .221 & .163 & .084 & .242 & .163 & .093 \\
Intervention & .155 & .130 & .073 & .160 & .130 & .075 & .167 & .131 & .078 & .164 & .132 & .077 \\
EI $\Delta$ & & & & -.135 & .122 & -.069 & -.150 & .135 & -.076 & -.138 & .136 & -.070 \\
z-SPS $\Delta$ & & & & & & & -.008 & .074 & -.008 & .021 & .076 & .020 \\
EI $\Delta$ X z-SPS $\Delta$ & & & & & & & & & & -.099 & .068 & -.093 \\
\hline
\end{tabular}

Note: EI= Ethnic Identity; SPS= Social Provisions Scale. All Sexual acts and consumption of alcohol and illegal substances refer to the last 30 days. ${ }^{*} p \leq 0.05 ; n=270$

Table Appendix 8.10

Regression Analysis for Moderating Variables Predicting SRAE-Decreased Nervousness Change

\begin{tabular}{lccccccccccccc} 
& \multicolumn{3}{c}{ Model 1 } & \multicolumn{1}{c}{ Model 2 } & \multicolumn{1}{c}{ Model 3 } \\
\hline Variable & $\beta$ & $S E$ & $B$ & $\beta$ & $S E$ & $B$ & $\beta$ & $S E$ & $B$ & $\beta$ & $S E$ & $B$ \\
\hline Gender & .033 & .140 & .015 & .020 & .141 & .009 & .033 & .143 & .015 & .035 & .143 & .016 \\
Length of Stay & .166 & .162 & .063 & .157 & .163 & .060 & .158 & .163 & .060 & .166 & .164 & .064 \\
Intervention & .159 & .130 & .075 & .163 & .131 & .076 & .176 & .131 & .082 & .184 & .132 & .086 & -.01 \\
EI $\Delta$ & & & & -.090 & .122 & -.046 & -.048 & .136 & -.025 & -.039 & .136 & -.020 \\
z-SPS $\Delta$ & & & & & & & -.081 & .074 & -.076 & -.066 & .077 & -.062 \\
EI $\Delta$ X z-SPS $\Delta$ & & & & & & & & & & -.066 & .068 & -.062 \\
\hline
\end{tabular}

Note: EI= Ethnic Identity; SPS= Social Provisions Scale. All Sexual acts and consumption of alcohol and illegal substances refer to the last 30 days. ${ }^{*} p \leq 0.05 ; n=270$ 
Table Appendix 8.11

Regression Analysis for Moderating Variables Predicting SRAE-Increased Risk Change

\begin{tabular}{|c|c|c|c|c|c|c|c|c|c|c|c|c|}
\hline & \multicolumn{3}{|c|}{ Model 1} & \multicolumn{3}{|c|}{ Model 2} & \multicolumn{3}{|c|}{ Model 3} & \multicolumn{3}{|c|}{ Model 4} \\
\hline Variable & $\beta$ & $S E$ & $B$ & $\beta$ & $S E$ & $B$ & $\beta$ & $S E$ & $B$ & $\beta$ & $S E$ & $B$ \\
\hline Gender & -.146 & .147 & -.064 & -.154 & .148 & -.068 & -.112 & .149 & -.049 & -.105 & .148 & -.046 \\
\hline Marital & .143 & .134 & .066 & .137 & .134 & .063 & .133 & .134 & .061 & .134 & .133 & .062 \\
\hline Education & .034 & .023 & .097 & .033 & .023 & .095 & .036 & .023 & .103 & .032 & .023 & .093 \\
\hline Length of Stay & -.174 & .166 & -.066 & -.180 & .166 & -.068 & -.163 & .166 & -.061 & -.135 & .166 & -.051 \\
\hline Intervention & .032 & .133 & .015 & .034 & .133 & .016 & .039 & .133 & .018 & .049 & .133 & .023 \\
\hline EI $\Delta$ & & & & -.069 & .124 & -.035 & -.138 & .138 & -.069 & -.117 & .137 & -.059 \\
\hline$z$-SPS $\Delta$ & & & & & & & .054 & .075 & .050 & .093 & .077 & .086 \\
\hline $\mathrm{EI} \Delta \mathrm{X} z-\operatorname{SPS} \Delta$ & & & & & & & & & & -.158 & .068 & -.148 \\
\hline
\end{tabular}

Note: EI= Ethnic Identity; SPS= Social Provisions Scale. All Sexual acts and consumption of alcohol and illegal substances refer to the last 30 days. ${ }^{*} p \leq 0.05 ; n=270$

Table Appendix 8.12

Regression Analysis for Moderating Variables Predicting Vaginal Episode Equivalent Change

\begin{tabular}{|c|c|c|c|c|c|c|c|c|c|c|c|c|}
\hline & \multicolumn{3}{|c|}{ Model 1} & \multicolumn{3}{|c|}{ Model 2} & \multicolumn{3}{|c|}{ Model 3} & \multicolumn{3}{|c|}{ Model 4} \\
\hline Variable & $\beta$ & $S E$ & $B$ & $\beta$ & $S E$ & $B$ & $\beta$ & $S E$ & $B$ & $\beta$ & $S E$ & $B$ \\
\hline Marital & -.280 & 1.625 & -.011 & -.352 & 1.629 & -.013 & -.312 & 1.645 & -.012 & -.413 & 1.652 & -.016 \\
\hline Intervention & .357 & 1.327 & .016 & .402 & 1.329 & .019 & .427 & 1.336 & .020 & .358 & 1.344 & .016 \\
\hline EI Belonging $\Delta$ & & & & -.766 & 1.028 & -.046 & -.787 & 1.168 & -.047 & -.871 & 1.175 & -.052 \\
\hline$z$-SPS $\Delta$ & & & & & & & -.070 & .758 & -.006 & -.198 & .825 & -.018 \\
\hline $\begin{array}{l}\text { EI Belonging } \Delta \mathrm{X} \\
z \text {-SPS } \Delta\end{array}$ & & & & & & & & & & .478 & .645 & .050 \\
\hline
\end{tabular}

Note: EI= Ethnic Identity; SPS= Social Provisions Scale. All Sexual acts and consumption of alcohol and illegal substances refer to the last 30 days. ${ }^{*} p \leq 0.05 ; n=270$ 
Table Appendix 8.13

Regression Analysis for Moderating Variables Predicting Partner Change

\begin{tabular}{|c|c|c|c|c|c|c|c|c|c|c|c|c|}
\hline & \multicolumn{3}{|c|}{ Model 1} & \multicolumn{3}{|c|}{ Model 2} & \multicolumn{3}{|c|}{ Model 3} & \multicolumn{3}{|c|}{ Model 4} \\
\hline Variable & $\beta$ & $S E$ & $B$ & $\beta$ & $S E$ & $B$ & $\beta$ & $S E$ & $B$ & $\beta$ & $S E$ & $B$ \\
\hline Length of Stay & -2.027 & 1.093 & -.112 & -2.092 & 1.094 & -.116 & -1.979 & 1.099 & -.110 & -1.957 & 1.106 & -.108 \\
\hline Intervention & -1.455 & .892 & -.099 & -1.414 & .893 & -.096 & -1.341 & .893 & -.091 & -1.343 & .900 & -.091 \\
\hline EI Belonging $\Delta$ & & & & -.692 & .691 & -.061 & -.745 & .780 & -.065 & -.739 & .786 & -.065 \\
\hline$z$-SPS $\Delta$ & & & & & & & -.214 & .507 & -.029 & -.158 & .552 & -.021 \\
\hline $\begin{array}{l}\text { EI Belonging } \Delta X \\
z \text {-SPS } \Delta\end{array}$ & & & & & & & & & & -.127 & .432 & -.019 \\
\hline
\end{tabular}

Note: EI= Ethnic Identity; SPS= Social Provisions Scale. All Sexual acts and consumption of alcohol and illegal substances refer to the last 30 days. ${ }^{*} p \leq 0.05 ; n=270$

Table Appendix 8.14

Regression Analysis for Moderating Variables Predicting Unprotected Partner Change

\begin{tabular}{|c|c|c|c|c|c|c|c|c|c|c|c|c|}
\hline & \multicolumn{3}{|c|}{ Model 1} & \multicolumn{3}{|c|}{ Model 2} & \multicolumn{3}{|c|}{ Model 3} & \multicolumn{3}{|c|}{ Model 4} \\
\hline Variable & $\beta$ & $S E$ & $B$ & $\beta$ & $S E$ & $B$ & $\beta$ & $S E$ & $B$ & $\beta$ & $S E$ & $B$ \\
\hline Length of Stay & -.043 & .262 & -.010 & -.055 & .263 & -.013 & .016 & .261 & .004 & .053 & .260 & .012 \\
\hline Intervention & -.218 & .214 & -.062 & -.211 & .214 & -.060 & -.194 & .212 & -.055 & -.183 & .212 & -.052 \\
\hline EI Belonging $\Delta$ & & & & -.124 & .166 & -.046 & -.244 & .185 & -.090 & -.224 & .185 & -.083 \\
\hline$z$-SPS $\Delta$ & & & & & & & .058 & .120 & .033 & .130 & .130 & .074 \\
\hline EI Belonging $\Delta \mathrm{X}$ & & & & & & & & & & -.197 & .102 & $-.128 *$ \\
\hline
\end{tabular}

Note: EI= Ethnic Identity; SPS= Social Provisions Scale. All Sexual acts and consumption of alcohol and illegal substances refer to the last 30 days.

$* p \leq 0.05 ; n=270$ 
Table Appendix 8.15

Regression Analysis for Moderating Variables Predicting Vaginal Acts without a Condom Change

\begin{tabular}{lccccccccccccc}
\hline & \multicolumn{3}{c}{ Model 1 } & \multicolumn{3}{c}{ Model 2 } & \multicolumn{3}{c}{ Model 3 } & \multicolumn{3}{c}{ Model 4 } \\
\hline Variable & $\beta$ & $S E$ & $B$ & $\beta$ & $S E$ & $B$ & $\beta$ & $S E$ & $B$ & $\beta$ & $S E$ & $B$ \\
\hline Gender & -.165 & .950 & -.011 & -.067 & .954 & -.005 & -.078 & .967 & -.005 & -.160 & .969 & -.011 \\
Marital & -1.012 & .862 & -.073 & -1.009 & .862 & -.073 & -1.007 & .865 & -.072 & -1.037 & .865 & -.075 \\
Education & .067 & .144 & .030 & .080 & .145 & .036 & .081 & .146 & .037 & .085 & .147 & .039 \\
Length of Stay & 1.146 & 1.103 & .066 & 1.196 & 1.104 & .069 & 1.207 & 1.113 & .069 & 1.184 & 1.117 & .068 \\
Intervention & 1.030 & .854 & .075 & .998 & .854 & .072 & 1.019 & .858 & .074 & 1.050 & .860 & .076 \\
EI Belonging $\Delta$ & & & & .711 & .667 & .067 & .828 & .749 & .078 & .863 & .754 & .081 \\
$z$-SPS $\Delta$ & & & & & & & -.216 & .486 & -.032 & -.531 & .530 & -.077 \\
EI Belonging $\Delta \mathrm{X}$ & & & & & & & & & & .591 & .411 & .098 \\
$z$-SPS $\Delta$ & & & & & & & & & & & & & \\
\hline
\end{tabular}

Note: EI= Ethnic Identity; SPS= Social Provisions Scale. All Sexual acts and consumption of alcohol and illegal substances refer to the last 30 days. ${ }^{*} p \leq 0.05 ; n=270$ 
Table Appendix 8.16

Regression Analysis for Moderating Variables Predicting Monthly Alcohol Consumption Change

\begin{tabular}{|c|c|c|c|c|c|c|c|c|c|c|c|c|}
\hline & \multicolumn{3}{|c|}{ Model 1} & \multicolumn{3}{|c|}{ Model 2} & \multicolumn{3}{|c|}{ Model 3} & \multicolumn{3}{|c|}{ Model 4} \\
\hline Variable & $\beta$ & $S E$ & $B$ & $\beta$ & $S E$ & $B$ & $\beta$ & $S E$ & $B$ & $\beta$ & $S E$ & $B$ \\
\hline $\begin{array}{l}\text { Length of } \\
\text { Stay }\end{array}$ & 26.090 & 12.043 & $.131^{*}$ & 27.756 & 11.964 & $.140 *$ & 26.890 & 12.025 & $.135^{*}$ & 27.282 & 12.092 & $.137 *$ \\
\hline Intervention & 3.208 & 9.834 & .020 & 2.165 & 9.763 & .013 & 3.109 & 9.766 & .019 & 3.367 & 9.838 & .021 \\
\hline $\begin{array}{l}\text { EI Belonging } \\
\Delta\end{array}$ & & & & 17.695 & 7.548 & $.141 *$ & 22.669 & 8.539 & $.181^{*}$ & 22.986 & 8.600 & $.184^{*}$ \\
\hline$z-\operatorname{SPS} \Delta$ & & & & & & & -8.445 & 5.544 & -.104 & -7.929 & 6.042 & -.098 \\
\hline $\begin{array}{l}\text { EI Belonging } \\
\Delta \mathrm{X} z \text {-SPS } \Delta\end{array}$ & & & & & & & & & & -1.873 & 4.723 & -.026 \\
\hline
\end{tabular}

Note: EI= Ethnic Identity; SPS= Social Provisions Scale. All Sexual acts and consumption of alcohol and illegal substances refer to the last 30 days. ${ }^{*} p \leq 0.05 ; n=270$ 
Table Appendix 8.17

Regression Analysis for Moderating Variables Predicting Monthly Marijuana Usage Change

\begin{tabular}{|c|c|c|c|c|c|c|c|c|c|c|c|c|}
\hline \multirow[b]{2}{*}{ Variable } & \multicolumn{3}{|c|}{ Model 1} & \multicolumn{3}{|c|}{ Model 2} & \multicolumn{3}{|c|}{ Model 3} & \multicolumn{3}{|c|}{ Model 4} \\
\hline & $\beta$ & $S E$ & $B$ & $\beta$ & $S E$ & $B$ & $\beta$ & $S E$ & $B$ & $\beta$ & $S E$ & $B$ \\
\hline Marital & 3.319 & 2.297 & .089 & 3.313 & 2.300 & .089 & 3.289 & 2.304 & .088 & 3.286 & 2.313 & .088 \\
\hline Education & .062 & .373 & .010 & .039 & .376 & .007 & .002 & .379 & .000 & -.009 & .383 & -.002 \\
\hline Length of Stay & .472 & 2.822 & .010 & .417 & 2.828 & .009 & .135 & 2.847 & .003 & .162 & 2.867 & .004 \\
\hline Intervention & 2.323 & 2.280 & .063 & 2.361 & 2.284 & .064 & 2.365 & 2.293 & .064 & 2.419 & 2.309 & .065 \\
\hline EI Belonging $\Delta$ & & & & -.878 & 1.771 & -.031 & -.035 & 2.001 & -.001 & .011 & 2.015 & .000 \\
\hline$z$-SPS $\Delta$ & & & & & & & -.957 & 1.297 & -.052 & -1.031 & 1.414 & -.056 \\
\hline $\begin{array}{l}\text { EI Belonging } \Delta \mathrm{X} z- \\
\text { SPS } \Delta\end{array}$ & & & & & & & & & & .012 & 1.106 & .001 \\
\hline
\end{tabular}

Note: EI= Ethnic Identity; SPS= Social Provisions Scale. All Sexual acts and consumption of alcohol and illegal substances refer to the last 30 days. $* x \leq 0.05 ; n=270$ 
Table Appendix 8.18

Regression Analysis for Moderating Variables Predicting Crack Usage Change

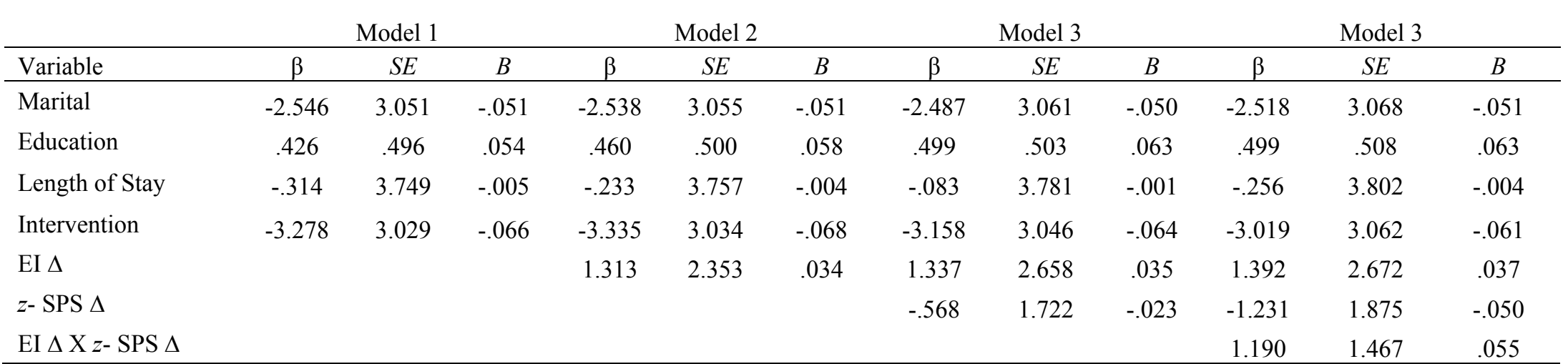

Note: EI= Ethnic Identity; SPS= Social Provisions Scale. All Sexual acts and consumption of alcohol and illegal substances refer to the last 30 days. ${ }^{*} p \leq 0.05 ; n=270$

Table Appendix 8.19

Regression Analysis for Moderating Variables Predicting SRAE Change

\begin{tabular}{|c|c|c|c|c|c|c|c|c|c|c|c|c|}
\hline & \multicolumn{3}{|c|}{ Model 1} & \multicolumn{3}{|c|}{ Model 2} & \multicolumn{3}{|c|}{ Model 3} & \multicolumn{3}{|c|}{ Model 4} \\
\hline Variable & $\beta$ & $S E$ & $B$ & $\beta$ & $S E$ & $B$ & $\beta$ & $S E$ & $B$ & $\beta$ & $S E$ & $B$ \\
\hline Gender & .034 & .121 & .017 & .021 & .122 & .011 & .033 & .124 & .017 & .038 & .124 & .019 \\
\hline Length of Stay & .196 & .140 & .087 & .192 & .140 & .085 & .194 & .141 & .085 & .205 & .142 & .090 \\
\hline Intervention & .119 & .113 & .064 & .123 & .113 & .066 & .130 & .113 & .070 & .129 & .114 & .070 \\
\hline EI Belonging $\Delta$ & & & & -.070 & .088 & -.049 & -.056 & .099 & -.039 & -.053 & .100 & -.037 \\
\hline$z$-SPS $\Delta$ & & & & & & & -.039 & .065 & -.042 & -.007 & .070 & -.007 \\
\hline $\begin{array}{l}\text { EI Belonging } \Delta \mathrm{X} z \text { - } \\
\text { SPS } \Delta\end{array}$ & & & & & & & & & & -.071 & .055 & -.087 \\
\hline
\end{tabular}

Note: EI= Ethnic Identity; SPS= Social Provisions Scale. All Sexual acts and consumption of alcohol and illegal substances refer to the last 30 days. ${ }^{*} p \leq 0.05 ; n=270$

Table Appendix 8.20

Regression Analysis for Moderating Variables Predicting SRAE-Enhanced Sex Change

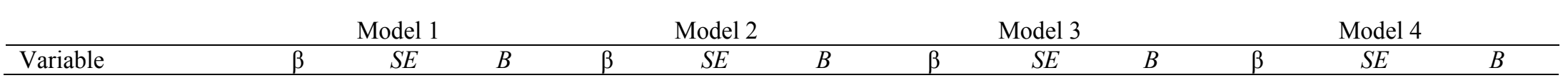




\begin{tabular}{lllllllllllll}
\hline Gender & .040 & .139 & .018 & .022 & .141 & .010 & .047 & .143 & .021 & .053 & .143 & .023 \\
Length of Stay & .224 & .162 & .086 & .218 & .162 & .083 & .228 & .163 & .087 & .240 & .163 & .092 \\
Intervention & .155 & .130 & .073 & .161 & .130 & .075 & .168 & .131 & .079 & .166 & .131 & .078 \\
EI Belonging $\Delta$ & & & & -.101 & .102 & -.061 & -.113 & .114 & -.068 & -.110 & .115 & -.067 \\
z-SPS $\Delta$ & & & & & & & -.011 & .074 & -.010 & .026 & .081 & .025 \\
EI Belonging $\Delta \mathrm{X} z$ - & & & & & & & & & & -.080 & .063 & -.084 \\
SPS $\Delta$ & & & & & & & & & \\
\hline
\end{tabular}

Note: EI= Ethnic Identity; SPS= Social Provisions Scale. All Sexual acts and consumption of alcohol and illegal substances refer to the last 30 days. ${ }^{*} p \leq 0.05 ; n=270$

Table Appendix 8.21

Regression Analysis for Moderating Variables Predicting SRAE-Decreased Nervousness Change

\begin{tabular}{|c|c|c|c|c|c|c|c|c|c|c|c|c|}
\hline & \multicolumn{3}{|c|}{ Model 1} & \multicolumn{3}{|c|}{ Model 2} & \multicolumn{3}{|c|}{ Model 3} & \multicolumn{3}{|c|}{ Model 4} \\
\hline Variable & $\beta$ & $S E$ & $B$ & $\beta$ & $S E$ & $B$ & $\beta$ & $S E$ & $B$ & $\beta$ & $S E$ & $B$ \\
\hline Gender & .033 & .140 & .015 & .021 & .141 & .009 & .034 & .143 & .015 & .039 & .143 & .017 \\
\hline Length of Stay & .166 & .162 & .063 & .162 & .162 & .062 & .160 & .163 & .061 & .172 & .163 & .066 \\
\hline Intervention & .159 & .130 & .075 & .163 & .131 & .076 & .176 & .131 & .082 & .178 & .131 & .083 \\
\hline EI Belonging $\Delta$ & & & & -.066 & .102 & -.040 & -.027 & .115 & -.016 & -.021 & .115 & -.013 \\
\hline$z-\operatorname{SPS} \Delta$ & & & & & & & -.084 & .074 & -.079 & -.057 & .081 & -.054 \\
\hline $\begin{array}{l}\text { EI Belonging } \Delta \mathrm{X} z- \\
\text { SPS }\end{array}$ & & & & & & & & & & -.068 & .063 & -.072 \\
\hline
\end{tabular}

Note: EI= Ethnic Identity; SPS= Social Provisions Scale. All Sexual acts and consumption of alcohol and illegal substances refer to the last 30 days. ${ }^{*} p \leq 0.05 ; n=270$ 
Table Appendix 8.22

Regression Analysis for Moderating Variables Predicting SRAE-Increased Risk Change

\begin{tabular}{|c|c|c|c|c|c|c|c|c|c|c|c|c|}
\hline & \multicolumn{3}{|c|}{ Model 1} & \multicolumn{3}{|c|}{ Model 2} & \multicolumn{3}{|c|}{ Model 3} & \multicolumn{3}{|c|}{ Model 4} \\
\hline Variable & $\beta$ & $S E$ & $B$ & $\beta$ & $S E$ & $B$ & $\beta$ & $S E$ & $B$ & $\beta$ & SE & $B$ \\
\hline Gender & -.146 & .147 & -.064 & -.133 & .148 & -.058 & -.103 & .149 & -.045 & -.086 & .148 & -.038 \\
\hline Marital & .143 & .134 & .066 & .143 & .134 & .066 & .146 & .134 & .067 & .149 & .133 & .069 \\
\hline Education & .034 & .023 & .097 & .036 & .023 & .102 & .038 & .023 & .108 & .034 & .023 & .096 \\
\hline Length of Stay & -.174 & .166 & -.066 & -.171 & .166 & -.064 & -.158 & .166 & -.060 & -.129 & .165 & -.049 \\
\hline Intervention & .032 & .133 & .015 & .028 & .133 & .013 & .037 & .133 & .017 & .033 & .132 & .015 \\
\hline EI Belonging $\Delta$ & & & & .087 & .104 & .052 & .063 & .116 & .037 & .069 & .116 & .041 \\
\hline$z-\operatorname{SPS} \Delta$ & & & & & & & .004 & .075 & .004 & .074 & .081 & .069 \\
\hline $\begin{array}{l}\text { EI Belonging } \Delta \mathrm{X} z \text { - } \\
\text { SPS } \Delta\end{array}$ & & & & & & & & & & -.159 & .063 & -.166 \\
\hline
\end{tabular}

Note: EI= Ethnic Identity; SPS= Social Provisions Scale. All Sexual acts and consumption of alcohol and illegal substances refer to the last 30 days. ${ }^{*} p \leq 0.05 ; n=270$

Table Appendix 8.23

Regression Analysis for Moderating Variables Predicting Vaginal Episode Equivalent Change

\begin{tabular}{lcccccccccccc} 
& \multicolumn{3}{c}{ Model 1 } & \multicolumn{1}{c}{ Model 2 } & \multicolumn{1}{c}{ Model 3 } \\
\hline Variable & $\beta$ & $S E$ & $B$ & $\beta$ & $S E$ & $B$ & $\beta$ & $S E$ & $B$ & $\beta$ & $S E$ & $B$ \\
\hline Marital & -2.421 & 1.327 & -.111 & -2.475 & 1.349 & -.113 & -2.454 & 1.356 & -.112 & -2.439 & 1.355 & -.112 \\
Intervention & .327 & 1.318 & .015 & .330 & 1.321 & .015 & .371 & 1.329 & .017 & .238 & 1.348 & .011 \\
EI Explore $\Delta$ & & & & -.265 & 1.107 & -.015 & -.194 & 1.156 & -.011 & -.426 & 1.163 & -.024 \\
z-SPS $\Delta$ & & & & & & & -.239 & .696 & -.022 & -.267 & .697 & -.025 \\
EI Explore $\Delta$ X z-SPS & & & & & & & & & & 1.108 & .669 & .103 \\
$\Delta$ & & & & & & & & & &
\end{tabular}

Note: EI= Ethnic Identity; SPS= Social Provisions Scale. All Sexual acts and consumption of alcohol and illegal substances refer to the last 30 days. ${ }^{*} p \leq 0.05 ; n=270$ 
Table Appendix 8.24

Multiple Linear Regression Analysis for Moderating Variables Predicting Partner Change

\begin{tabular}{|c|c|c|c|c|c|c|c|c|c|c|c|c|}
\hline \multirow[b]{2}{*}{ Variable } & \multicolumn{3}{|c|}{ Model 1} & \multicolumn{3}{|c|}{ Model 2} & \multicolumn{3}{|c|}{ Model 3} & \multicolumn{3}{|c|}{ Model 4} \\
\hline & $\beta$ & $S E$ & $B$ & $\beta$ & $S E$ & $B$ & $\beta$ & $S E$ & $B$ & $\beta$ & $S E$ & $B$ \\
\hline Length of Stay & -2.027 & 1.093 & -.112 & -2.068 & 1.100 & -.115 & -1.996 & 1.104 & -.111 & -1.891 & 1.124 & -.105 \\
\hline Intervention & -1.455 & .892 & -.099 & -1.451 & .894 & -.098 & -1.362 & .894 & -.092 & -1.368 & .910 & -.093 \\
\hline EI Explore $\Delta$ & & & & -.264 & .742 & -.022 & -.201 & .767 & -.016 & -.141 & .776 & -.012 \\
\hline$z-\operatorname{SPS} \Delta$ & & & & & & & -.399 & .469 & -.054 & -.382 & .471 & -.052 \\
\hline $\begin{array}{l}\text { EI Explore } \Delta X z- \\
\text { SPS } \Delta\end{array}$ & & & & & & & & & & -.267 & .453 & -.036 \\
\hline
\end{tabular}

Note: EI= Ethnic Identity; SPS= Social Provisions Scale. All Sexual acts and consumption of alcohol and illegal substances refer to the last 30 days. ${ }^{*} p \leq 0.05 ; n=270$

Table Appendix 8.25

Regression Analysis for Moderating Variables Predicting Unprotected Partner Change

\begin{tabular}{|c|c|c|c|c|c|c|c|c|c|c|c|c|}
\hline \multirow[b]{2}{*}{ Variable } & \multicolumn{3}{|c|}{ Model 1} & \multicolumn{3}{|c|}{ Model 2} & \multicolumn{3}{|c|}{ Model 3} & \multicolumn{3}{|c|}{ Model 4} \\
\hline & $\beta$ & $S E$ & $B$ & $\beta$ & $S E$ & $B$ & $\beta$ & $S E$ & $B$ & $\beta$ & $S E$ & $B$ \\
\hline Length of Stay & -.043 & .262 & -.010 & .003 & .262 & .001 & .048 & .261 & .011 & .061 & .265 & .014 \\
\hline Intervention & -.218 & .214 & -.062 & -.222 & .213 & -.063 & -.200 & .212 & -.057 & -.161 & .215 & -.046 \\
\hline EI $\Delta$ & & & & .298 & .177 & .103 & .282 & .182 & .098 & .318 & .183 & $.110^{* *}$ \\
\hline$z-\operatorname{SPS} \Delta$ & & & & & & & -.055 & .111 & -.032 & -.054 & .111 & -.031 \\
\hline $\begin{array}{l}\text { EI Explore } \Delta \mathrm{X} z \text { - } \\
\text { SPS } \Delta\end{array}$ & & & & & & & & & & -.161 & .107 & -.092 \\
\hline
\end{tabular}

Note: EI= Ethnic Identity; SPS= Social Provisions Scale. All Sexual acts and consumption of alcohol and illegal substances refer to the last 30 days. ${ }^{*} p \leq 0.05 ; n=270$ 
Table Appendix 8.26

Regression Analysis for Moderating Variables Predicting Unprotected Vaginal Acts

\begin{tabular}{|c|c|c|c|c|c|c|c|c|c|c|c|c|}
\hline \multirow[b]{2}{*}{ Variable } & \multicolumn{3}{|c|}{ Model 1} & \multicolumn{3}{|c|}{ Model 2} & \multicolumn{3}{|c|}{ Model 3} & \multicolumn{3}{|c|}{ Model 4} \\
\hline & $\beta$ & $S E$ & $B$ & $\beta$ & $S E$ & $B$ & $\beta$ & $S E$ & $B$ & $\beta$ & $S E$ & $B$ \\
\hline Gender & -.165 & .950 & -.011 & -.081 & .946 & -.006 & -.091 & .962 & -.006 & -.033 & .954 & -.002 \\
\hline Marital & -1.012 & .862 & -.073 & -.737 & .869 & -.053 & -.718 & .873 & -.052 & -.710 & .865 & -.051 \\
\hline Education & .067 & .144 & .030 & .071 & .144 & .032 & .072 & .145 & .032 & .089 & .144 & .040 \\
\hline Length of Stay & 1.146 & 1.103 & .066 & 1.464 & 1.110 & .084 & 1.498 & 1.119 & .086 & 1.366 & 1.110 & .079 \\
\hline Intervention & 1.030 & .854 & .075 & .990 & .850 & .072 & 1.017 & .854 & .074 & 1.028 & .857 & .075 \\
\hline EI Explore $\Delta$ & & & & 1.401 & .729 & .121 & 1.487 & .759 & .128 & 1.494 & .761 & .129 \\
\hline$z$-SPS $\Delta$ & & & & & & & -.220 & .453 & -.032 & -.339 & .453 & -.049 \\
\hline $\begin{array}{l}\text { EI Explore } \Delta X z \text { - } \\
\text { SPS } \Delta\end{array}$ & & & & & & & & & & .939 & .430 & .137 \\
\hline
\end{tabular}

Note: EI= Ethnic Identity; SPS= Social Provisions Scale. All Sexual acts and consumption of alcohol and illegal substances refer to the last 30 days. ${ }^{*} p \leq 0.05 ; n=270$ 
Table Appendix 8.27

Regression Analysis for Moderating Variables Predicting Monthly Alcohol Consumption Change

\begin{tabular}{|c|c|c|c|c|c|c|c|c|c|c|c|c|}
\hline & \multicolumn{3}{|c|}{ Model 1} & \multicolumn{3}{|c|}{ Model 2} & \multicolumn{3}{|c|}{ Model 3} & \multicolumn{3}{|c|}{ Model 4} \\
\hline Variable & $\beta$ & $S E$ & $B$ & $\beta$ & $S E$ & $B$ & $\beta$ & $S E$ & $B$ & $\beta$ & $S E$ & $B$ \\
\hline Length of Stay & 26.090 & 12.043 & .131 & 31.558 & 11.692 & .159 & 30.918 & 11.741 & $.156^{*}$ & 29.384 & 11.946 & $.148^{*}$ \\
\hline Intervention & 3.208 & 9.834 & .020 & 2.755 & 9.497 & .017 & 3.882 & 9.506 & .024 & 4.983 & 9.679 & .031 \\
\hline EI Explore $\Delta$ & & & & 35.561 & 7.887 & .266 & 38.198 & 8.155 & $.285^{*}$ & 38.075 & 8.247 & $.284 *$ \\
\hline$z$-SPS $\Delta$ & & & & & & & -7.646 & 4.985 & -.095 & -7.930 & 5.011 & -.098 \\
\hline EI Explore $\Delta \mathrm{X}$ & & & & & & & & & & .588 & 4.820 & .007 \\
\hline
\end{tabular}

Note: EI= Ethnic Identity; SPS= Social Provisions Scale. All Sexual acts and consumption of alcohol and illegal substances refer to the last 30 days. ${ }^{*} p \leq 0.05 ; n=270$

Table Appendix 8.28

Regression Analysis for Moderating Variables Predicting Marijuana Usage Change

\begin{tabular}{|c|c|c|c|c|c|c|c|c|c|c|c|c|}
\hline & \multicolumn{3}{|c|}{ Model 1} & \multicolumn{3}{|c|}{ Model 2} & \multicolumn{3}{|c|}{ Model 3} & \multicolumn{3}{|c|}{ Model 4} \\
\hline Variable & $\beta$ & $S E$ & $B$ & $\beta$ & $S E$ & $B$ & $\beta$ & $S E$ & $B$ & $\beta$ & $\mathrm{SE}$ & $B$ \\
\hline Marital & 3.319 & 2.297 & .089 & 3.215 & 2.339 & .086 & 3.300 & 2.345 & .088 & 3.313 & 2.355 & .089 \\
\hline Education & .062 & .373 & .010 & .059 & .374 & .010 & .003 & .378 & .000 & .001 & .380 & .000 \\
\hline Length of Stay & .472 & 2.822 & .010 & .395 & 2.844 & .009 & .141 & 2.858 & .003 & .129 & 2.913 & .003 \\
\hline Intervention & 2.323 & 2.280 & .063 & 2.325 & 2.284 & .063 & 2.365 & 2.293 & .064 & 2.473 & 2.338 & .067 \\
\hline EI Explore $\Delta$ & & & & -.472 & 1.919 & -.015 & .049 & 1.992 & .002 & .121 & 2.016 & .004 \\
\hline$z$-SPS $\Delta$ & & & & & & & -.975 & 1.202 & -.053 & -.982 & 1.210 & -.053 \\
\hline $\begin{array}{l}\text { EI Explore } \Delta \mathrm{X} \\
z \text {-SPS } \Delta\end{array}$ & & & & & & & & & & -.312 & 1.160 & -.017 \\
\hline
\end{tabular}

Note: EI= Ethnic Identity; SPS= Social Provisions Scale. All Sexual acts and consumption of alcohol and illegal substances refer to the last 30 days. ${ }^{*} p \leq 0.05 ; n=270$ 
Table Appendix 8.29

Regression Analysis for Moderating Variables Predicting Crack Usage Change

\begin{tabular}{|c|c|c|c|c|c|c|c|c|c|c|c|c|}
\hline & \multicolumn{3}{|c|}{ Model 1} & \multicolumn{3}{|c|}{ Model 2} & \multicolumn{3}{|c|}{ Model 3} & \multicolumn{3}{|c|}{ Model 4} \\
\hline Variable & $\beta$ & $S E$ & $B$ & $\beta$ & $S E$ & $B$ & $\beta$ & SE & $B$ & $\beta$ & $\mathrm{SE}$ & $B$ \\
\hline Marital & -2.546 & 3.051 & -.051 & -2.735 & 3.107 & -.055 & -2.726 & 3.115 & -.055 & -2.903 & 3.113 & -.058 \\
\hline Education & .426 & .496 & .054 & .421 & .497 & .053 & .479 & .502 & .061 & .444 & .503 & .056 \\
\hline $\begin{array}{l}\text { Length of } \\
\text { Stay }\end{array}$ & -.314 & 3.749 & -.005 & -.453 & 3.778 & -.008 & -.204 & 3.796 & -.003 & .753 & 3.851 & .012 \\
\hline Intervention & -3.278 & 3.029 & -.066 & -3.273 & 3.034 & -.066 & -3.141 & 3.046 & -.064 & -3.976 & 3.090 & -.081 \\
\hline EI Explore $\Delta$ & & & & -.861 & 2.549 & -.021 & -1.076 & 2.646 & -.026 & -1.131 & 2.665 & -.028 \\
\hline$z$-SPS $\Delta$ & & & & & & & -.022 & 1.597 & -.001 & .150 & 1.599 & .006 \\
\hline $\begin{array}{l}\text { EI Explore } \Delta \\
\text { X } z \text {-SPS } \Delta\end{array}$ & & & & & & & & & & .105 & 1.533 & .004 \\
\hline
\end{tabular}

the last 30 days. ${ }^{*} p \leq 0.05 ; n=270$ 
Table Appendix 8.30

Regression Analysis for Moderating Variables Predicting SRAE Change

\begin{tabular}{lccccccccccccc}
\hline & \multicolumn{3}{c}{ Model 1 } & \multicolumn{1}{c}{ Model 2 } & \multicolumn{4}{c}{ Model 3 } & \multicolumn{2}{c}{ Model 4 } \\
\hline Variable & $\beta$ & $S E$ & $B$ & $\beta$ & $S E$ & $B$ & $\beta$ & $S E$ & $B$ & $\beta$ & SE & $B$ \\
\hline Gender & .034 & .121 & .017 & .024 & .121 & .012 & .033 & .124 & .017 & .026 & .125 & .013 \\
Length of Stay & .196 & .140 & .087 & .180 & .141 & .079 & .182 & .141 & .080 & .199 & .144 & .088 \\
Intervention & .119 & .113 & .064 & .121 & .113 & .065 & .128 & .113 & .069 & .130 & .115 & .070 \\
EI Explore $\Delta$ & & & & -.118 & .094 & -.077 & -.109 & .097 & -.071 & -.098 & .098 & -.064 \\
$z$-SPS $\Delta$ & & & & & & & -.038 & .060 & -.041 & -.036 & .060 & -.039 \\
EI Explore $\Delta \mathrm{X}$ & & & & & & & & & & -.050 & .057 & -.054
\end{tabular}

Note: EI= Ethnic Identity; SPS= Social Provisions Scale. All Sexual acts and consumption of alcohol and illegal substances refer to the last 30 days. ${ }^{*} p \leq 0.05 ; n=270$

Table Appendix 8.31

Regression Analysis for Moderating Variables Predicting SRAE-Enhanced Sex Change

\begin{tabular}{|c|c|c|c|c|c|c|c|c|c|c|c|c|}
\hline & \multicolumn{3}{|c|}{ Model 1} & \multicolumn{3}{|c|}{ Model 2} & \multicolumn{3}{|c|}{ Model 3} & \multicolumn{3}{|c|}{ Model 4} \\
\hline Variable & $\beta$ & $S E$ & $B$ & $\beta$ & $S E$ & $B$ & $\beta$ & $S E$ & $B$ & $\beta$ & $S E$ & $B$ \\
\hline Gender & .040 & .139 & .018 & .032 & .140 & .014 & .053 & .143 & .023 & .034 & .143 & .015 \\
\hline Length of Stay & .224 & .162 & .086 & .211 & .162 & .080 & .217 & .163 & .083 & .259 & .166 & .099 \\
\hline Intervention & .155 & .130 & .073 & .156 & .130 & .073 & .165 & .131 & .077 & .162 & .133 & .076 \\
\hline EI Explore $\Delta$ & & & & -.097 & .108 & -.055 & -.095 & .112 & -.054 & -.073 & .113 & -.041 \\
\hline$z$-SPS $\Delta$ & & & & & & & -.029 & .069 & -.027 & -.023 & .069 & -.022 \\
\hline EI Explore $\Delta \mathrm{X} z$ - & & & & & & & & & & -.101 & .066 & -.095 \\
\hline
\end{tabular}

Note: EI= Ethnic Identity; SPS= Social Provisions Scale. All Sexual acts and consumption of alcohol and illegal substances refer to the last 30 days. ${ }^{*} p \leq 0.05 ; n=270$ 
Table Appendix 8.32

Regression Analysis for Moderating Variables Predicting SRAE-Decreased Nervousness Change

\begin{tabular}{|c|c|c|c|c|c|c|c|c|c|c|c|c|}
\hline \multirow[b]{2}{*}{ Variable } & \multicolumn{3}{|c|}{ Model 1} & \multicolumn{3}{|c|}{ Model 2} & \multicolumn{3}{|c|}{ Model 3} & \multicolumn{3}{|c|}{ Model 4} \\
\hline & $\beta$ & $S E$ & $B$ & $\beta$ & $S E$ & $B$ & $\beta$ & $S E$ & $B$ & $\beta$ & $S E$ & $B$ \\
\hline Gender & .033 & .140 & .015 & .027 & .140 & .012 & .035 & .143 & .015 & .033 & .144 & .015 \\
\hline Length of Stay & .166 & .162 & .063 & .156 & .163 & .060 & .156 & .163 & .059 & .160 & .166 & .061 \\
\hline Intervention & .159 & .130 & .075 & .160 & .131 & .075 & .175 & .131 & .082 & .186 & .133 & .087 \\
\hline EI Explore $\Delta$ & & & & -.068 & .109 & -.038 & -.043 & .112 & -.024 & -.033 & .113 & -.019 \\
\hline$z$-SPS $\Delta$ & & & & & & & -.085 & .069 & -.080 & -.085 & .069 & -.080 \\
\hline $\begin{array}{l}\text { EI Explore } \Delta \mathrm{X} z- \\
\text { SPS } \Delta\end{array}$ & & & & & & & & & & -.046 & .066 & -.043 \\
\hline
\end{tabular}

Note: EI= Ethnic Identity; SPS= Social Provisions Scale. All Sexual acts and consumption of alcohol and illegal substances refer to the last 30 days. ${ }^{*} p \leq 0.05 ; n=270$ 
Table Appendix 8.33

Regression Analysis for Moderating Variables Predicting SRAE-Increased Risk Change

\begin{tabular}{|c|c|c|c|c|c|c|c|c|c|c|c|c|}
\hline \multirow[b]{2}{*}{ Variable } & \multicolumn{3}{|c|}{ Model 1} & \multicolumn{3}{|c|}{ Model 2} & \multicolumn{3}{|c|}{ Model 3} & \multicolumn{3}{|c|}{ Model 4} \\
\hline & $\beta$ & $S E$ & $B$ & $\beta$ & $S E$ & $B$ & $\beta$ & $S E$ & $B$ & $\beta$ & $\mathrm{SE}$ & $B$ \\
\hline Gender & -.146 & .147 & -.064 & -.169 & .146 & -.074 & -.117 & .147 & -.051 & -.118 & .147 & -.052 \\
\hline Marital & .143 & .134 & .066 & .082 & .135 & .038 & .077 & .134 & .035 & .079 & .134 & .036 \\
\hline Education & .034 & .023 & .097 & .033 & .022 & .095 & .037 & .022 & .106 & .036 & .022 & .103 \\
\hline Length of Stay & -.174 & .166 & -.066 & -.216 & .165 & -.082 & -.196 & .164 & -.074 & -.187 & .167 & -.071 \\
\hline Intervention & .032 & .133 & .015 & .034 & .131 & .016 & .035 & .131 & .016 & .061 & .133 & .028 \\
\hline EI Explore $\Delta$ & & & & -.277 & .111 & $-.155^{*}$ & -.320 & .114 & $-.179 *$ & -.296 & .115 & $-.165 *$ \\
\hline$z-\operatorname{SPS} \Delta$ & & & & & & & .070 & .069 & .065 & .070 & .069 & .065 \\
\hline $\begin{array}{l}\text { EI Explore } \Delta \\
\mathrm{X} z \text {-SPS } \Delta\end{array}$ & & & & & & & & & & -.105 & .066 & -.098 \\
\hline
\end{tabular}

Note: EI= Ethnic Identity; SPS= Social Provisions Scale. All Sexual acts and consumption of alcohol and illegal substances refer to the last 30 days. ${ }^{*} p \leq 0.05 ; n=270$ 
VITA

\section{NANCY SHEHADEH}

May 12, 1978

1997-2000

2001-2002

2002

2002

$2010-2012$

2008 - Present

2010 - Present

2011
Born, Queens, New York

B.A., Health Administration

Florida Atlantic University

Boca Raton, Florida

Master of Public Health

Certificate of Health Promotion

Florida International University

Miami, Florida

CHES (Certified Health Education Specialist) certificate

Award for academic achievement

Florida International University

Miami, Florida

Doctoral Candidate in Public Health

Florida International University

Miami, Florida

Research Assistant

HIV/AIDS- Migrant Worker Project in Immokalee Florida

H. V. McCoy, $\mathrm{PhD}$

Florida International University

Miami, Florida

Section Editor

California Journal of Health Promotion

Section: HIV and Sexually Transmitted Infections

Abstract Reviewer

American Psychological Health Association Conference 


\section{PUBLICATIONS AND PRESENTATIONS}

McCoy, H.V., Rubens, M., \& Shehadeh, N. (April 2011). Access to Health Care Services: Impact of Ethnic Identity among Migrant Workers. Poster session at the Highlights of "From Disparities Research to Disparities Interventions" Conference, Arlington, Virginia.

McCoy, H. V., Shehadeh, N., Winter, K., \& Balkrishnan Rubens, M. (2010). Among Migrant Workers, Newcomer Status may be a Protective Factor for HIV/STDs. Poster session at the International AIDS Conference, Vienna, Austria.

N. Shehadeh, H. V. McCoy, M. B. Rubens. (2011). Factors that Positively Influence Self Efficacy Change among Migrant Workers. Poster session at the American Public Health Association Conference, Washington, D.C.

N. Shehadeh, H. V. McCoy, M. B. Rubens. (2011). Protective HIV Risk Behaviors: Predictors of Condom Carrying Behavior among Migrant Workers. Poster session at the American Public Health Association Conference, Washington, D.C.

Renfrew, R., McCoy, H. V., Rubens, M., Shehadeh, N., \& Winter, K. (2010). Sexual Risk Behavior and Drug Use among Migrant Workers. Oral presentation at the American Public Health Association Conference, Denver, Colorado.

Shehadeh, N. (2010). The Ever-evolving concept of a New Public Health. The California Journal of Health Promotion. 8(1), 82-87.

Shehadeh, N., McCoy, H. V., \& Winter, K. (November 2010). HIV and STI's Risk Differences by Gender and Ethnicity among Migrant Workers. Poster session at the Women, Gender, \& Sexuality Student Conference, Florida International University, Miami, Florida.

Shehadeh, N., McCoy, H. V., Rubens, M. B., Winter, K. \& Renfrew, R. (2010). Ethnic Identity: Impact on Sexual Behavior among Migrant Workers. Poster session at the American Public Health Association Conference, Denver, Colorado.

Shehadeh, N., McCoy, H.V., Rubens, M., Batra, A., Renfrew, R., and Winter, K. (2012). The Impact of Ethnic Identity on Changes in High Risk HIV Behaviors in Sexually Active Migrant Workers. Journal of Immigrant and Minority Health, 14(1), 100-106. 